\title{
Copyright
}

by

Rebecca Lee Vincelette

2009 
The Dissertation Committee for Rebecca Lee Vincelette Certifies that this is the approved version of the following dissertation:

\section{THERMAL LENSING IN OCULAR MEDIA}

\section{Committee:}

Ashley J. Welch, Supervisor

Benjamin A. Rockwell, Co-Supervisor

Robert J. Thomas

Massoud M. Motamedi

H. Grady Rylander, III

Thomas E. Milner 


\title{
THERMAL LENSING IN OCULAR MEDIA
}

\author{
by \\ Rebecca Lee Vincelette, B.S., M.S. \\ Dissertation \\ Presented to the Faculty of the Graduate School of \\ The University of Texas at Austin \\ in Partial Fulfillment \\ of the Requirements \\ for the Degree of
}

Doctor of Philosophy

The University of Texas at Austin

May, 2009 
Dedicated to Grandma, Carol Nadine Vincelette 


\section{Acknowledgements}

Every student is the result of a sum of efforts from numerous teachers. There are teachers who stand out and leave a lifelong impression on his/her students. I would like to use this section to acknowledge these teachers, beginning with Dr. Ashley J. Welch, my Ph.D. supervisor. I would like to thank Dr. A.J. Welch for his challenging questions, guidance and invaluable feedback. His commitment to scientific research and working collaborations with the United States Air Force afforded me the unique opportunity for this work. I will forever be grateful for Dr. Welch's patience, gentle guidance and mentorship.

My dissertation work supports the research efforts at the Air Force Research Lab (AFRL) under the supervision of Dr. Robert (Bob) Thomas and Dr. Ben Rockwell. Both Dr.'s Thomas and Rockwell have been instrumental in teaching me about optical modeling and experimental research. I am very grateful for the valuable mentorship they have provided me in the Air Force Lab.

Many scientists have thanked Mr. Gary Noojin over several years at the Air Force Lab, Optical Radiation Branch. He is a walking encyclopedia of information on anything laser, electronic, imaging, and/or Labview related. Though he can bewilder a new graduate student by offering multiple solutions to a single problem, he never steered me wrong and always took the time to assist in my moments of frustration and confusion. I am so thankful for all his assistance and enthusiastic, scientific discussions which inspired me to try new experimental techniques over the years with AFRL. 
I would never have considered moving to Texas if it were not for my undergraduate Electrical Engineering advisor, Dr. Steve Barrett, Associate Professor, University of Wyoming, USAF Col. (retired). His mentorship led me to UT and Dr. Welch's laboratory. I would also like to thank my undergraduate Chemistry advisors, Dr. Robert (Bob) Corcoran and Dr. Jeff Yarger; both mentors were catalysts in my journey to optical research. My gratitude also goes to Ms. Cynthia Fite, Mrs. Sue McNamee and Mr. Larry Cattell from Green Mountain High School, Lakewood, CO, who challenged and inspired me to take those first steps towards becoming a biomedical engineer.

Additionally, I would like to thank Chris Humphrey for all her help to keep things moving like a well-oiled machine. Last, but certainly not least, I am indebted to the love and support I have received from my family throughout every step of the way.

Support for this research was provided by the Consortium Research Fellowship Program at the Air Force Research Laboratory, Brooks City-Base, San Antonio, TX under contract number FA8650-05-2-6501. 


\section{THERMAL LENSING IN OCULAR MEDIA}

Publication No.

Rebecca Lee Vincelette, Ph.D.

The University of Texas at Austin, 2009

Supervisor: Ashley J. Welch

Co-Supervisor: Benjamin A. Rockwell

This research was a collaborative effort between the Air Force Research Laboratory (AFRL) and the University of Texas to examine the laser-tissue interaction of thermal lensing induced by continuous-wave, CW, near-infrared, NIR, laser radiation in the eye and its influence on the formation of a retinal lesion from said radiation. $\mathrm{CW}$ NIR laser radiation can lead to a thermal lesion induced on the retina given sufficient power and exposure duration as related to three basic parameters; the percent of transmitted energy to, the optical absorption of, and the size of the laser-beam created at the retina. Thermal lensing is a well-known phenomenon arising from the optical absorption, and subsequent temperature rise, along the path of the propagating beam through a medium. Thermal lensing causes the laser-beam profile delivered to the retina to be time dependent. Analysis of a dual-beam, multidimensional, high-frame rate, confocal imaging system in an artificial eye determined the rate of thermal lensing in aqueous media exposed to $1110,1130,1150$ and $1318-\mathrm{nm}$ wavelengths was related to the 
power density created along the optical axis and linear absorption coefficient of the medium. An adaptive optics imaging system was used to record the aberrations induced by the thermal lens at the retina in an artificial eye during steady-state. Though the laserbeam profiles changed over the exposure time, the CW NIR retinal damage thresholds between 1110-1319-nm were determined to follow conventional fitting algorithms which neglected thermal lensing. A first-order mathematical model of thermal lensing was developed by conjoining an ABCD beam propagation method, Beer's law of attenuation, and a solution to the heat-equation with respect to radial diffusion. The model predicted that thermal lensing would be strongest for small $(<4-\mathrm{mm}) 1 / \mathrm{e}^{2}$ laser-beam diameters input at the corneal plane and weakly transmitted wavelengths where less than $5 \%$ of the energy is delivered to the retina. The model predicted thermal lensing would cause the retinal damage threshold for wavelengths above 1300-nm to increase with decreasing beam-diameters delivered to the corneal plane, a behavior which was opposite of equivalent conditions simulated without thermal lensing. 


\section{Table of Contents}

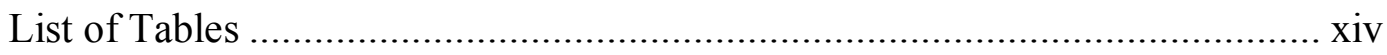

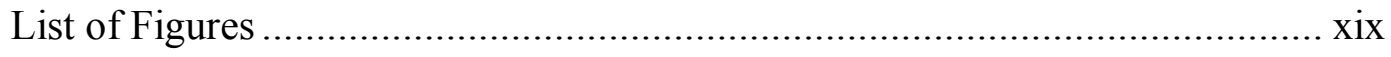

$\begin{array}{ll}\text { CHAPTER } 1 & 1\end{array}$

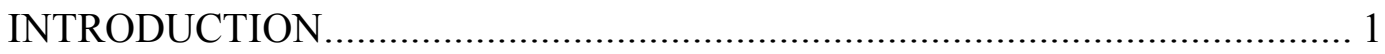

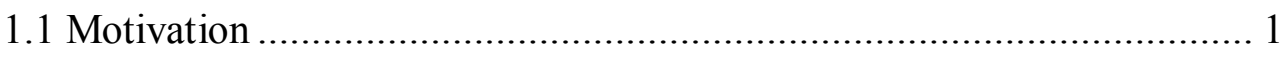

1.2 Dissertation Overview and Specific Aims ............................................ 4

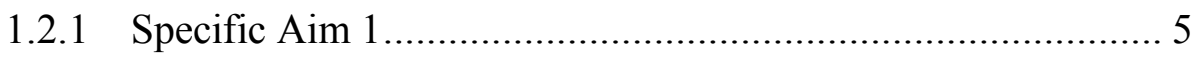

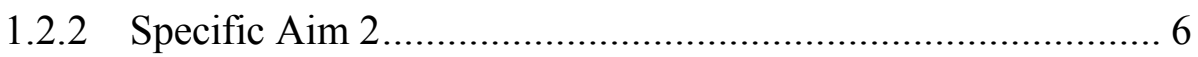

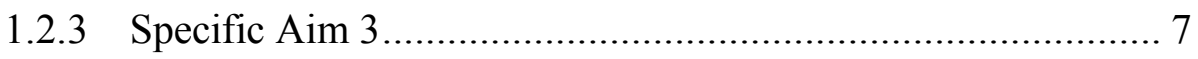

1.2.4 Specific Aim 4................................................................ 7

1.2.5 Specific Aim 5 5.............................................................. 7

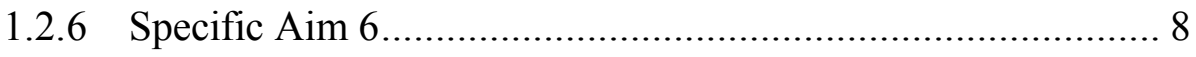

$\begin{array}{lr}\text { CHAPTER } 2 & 9\end{array}$

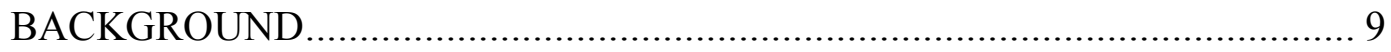

2.1 Review of Corneal and Retinal Threshold Studies................................. 9

2.1.1 Standard Procedures in Ocular Damage Threshold Studies ........ 9

2.1.2 General Classes of Retinal Injury ……………...................... 11

2.1.3 Summary of Corneal and Retinal Threshold Studies (1300-1356 nm),

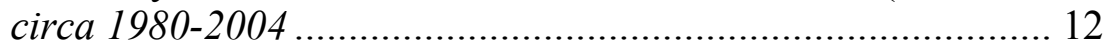

2.1.4 Summary of Histological Findings ........................................... 14

2.2 Retinal Damage Threshold Action Spectrum...................................... 17

2.2.1 Criteria 1: Absorbance of the RPE and Water Content of the Retina in the NIR Wavelength Region.............................................. 19

2.2.2 Criteria 2: Transmission of Laser Radiation to the Retina ........ 22

2.2.3 Criteria 3: Prediction of Spot Size Using Chromatic Dispersion

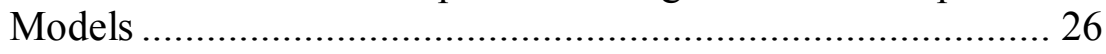

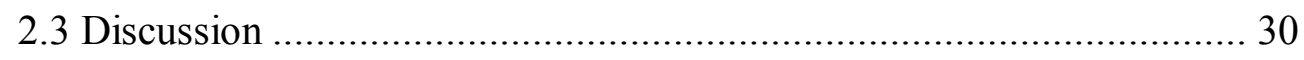




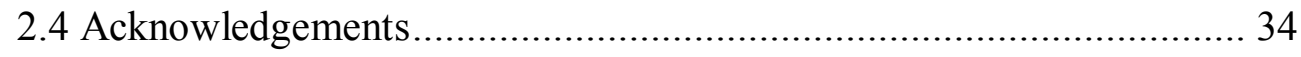

$\begin{array}{ll}\text { CHAPTER } 3 & 35\end{array}$

CONFOCAL IMAGING OF THE THERMAL LENSING EFFECT IN AN

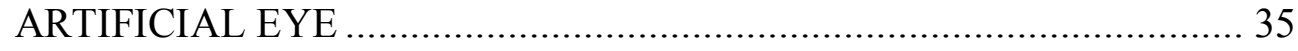

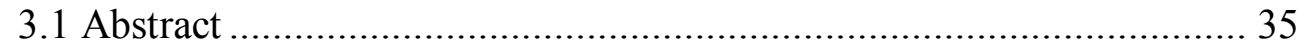

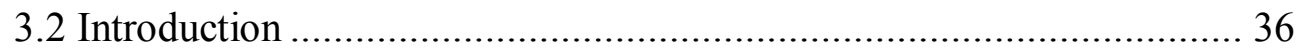

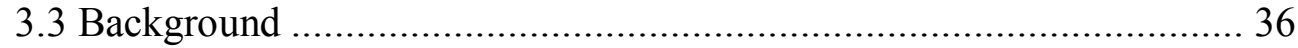

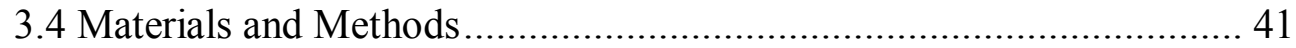

3.4.1 Thermal Lens Investigation Using a Modified Artificial Eye ... 41

3.4.2 Thermal Lens Investigation Using the Cain-Cell Artificial Eye 45

3.4.3 Timing Hardware, Data Acquisition and Analysis .................. 48

3.4.4 Reduction of Multidimensional Data ........................................ 50

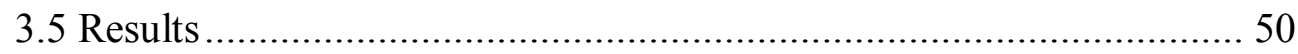

3.5.1 Thermal Lensing of Vitreous and Water in the Modified Artificial Eye ................................................................................. 50

3.5.2 Thermal Lensing in the Water-Filled Cain-Cell Artificial Eye . 54

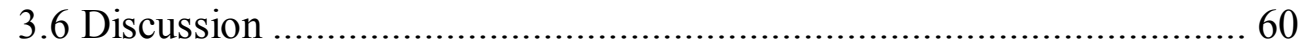

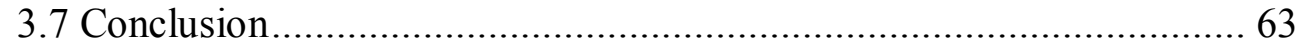

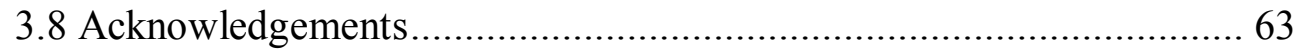

$\begin{array}{lr}\text { CHAPTER } 4 & 64\end{array}$

A FIRST-ORDER MODEL OF THERMAL LENSING IN A VIRTUAL EYE. 64

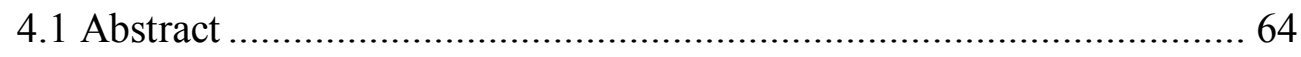

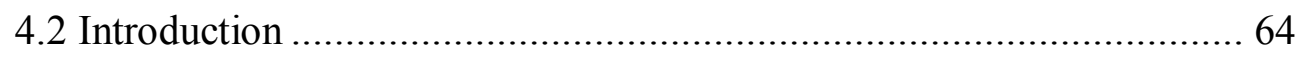

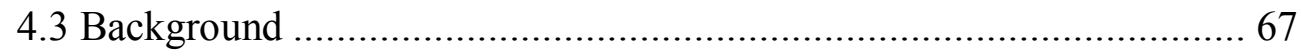

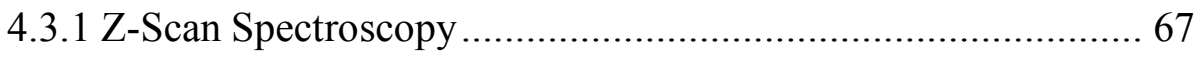

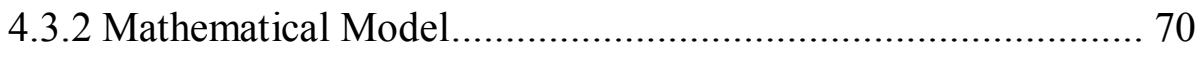

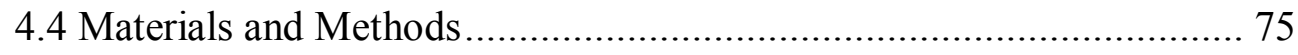

4.4.1 Single-Beam, Closed-Aperture Z-Scan .................................... 75

4.4.2 Model of the Virtual Eye .......................................................... 77

4.4.3 First-Order Thermal Lensing Mathematical Model .................. 79 


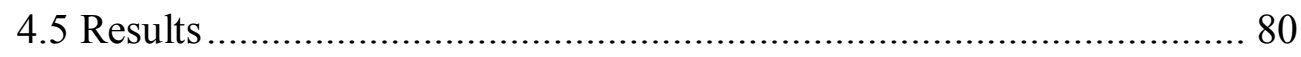

4.5.1 Model Compared to Experimental Z-Scan Data.................... 80

4.5.2 Thermal Lensing Model Trends in the Virtual Eye ............... 82

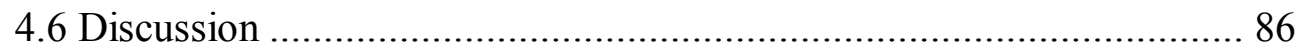

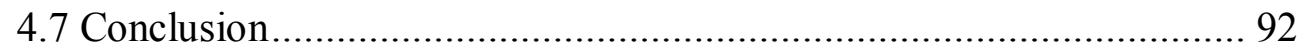

4.8 Acknowledgements......................................................................... 93

$\begin{array}{lr}\text { CHAPTER } 5 & 94\end{array}$

TRENDS IN RETINAL DAMAGE THRESHOLDS FROM CONTINUOUS-WAVE NEAR-INFRARED LASER RADIATION: a study at 1110, 1130, 1150 and $1319 \mathrm{~nm}$

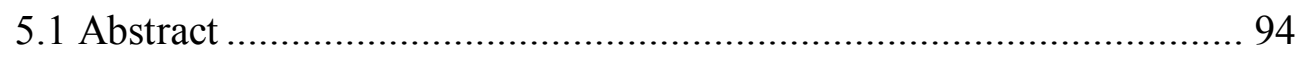

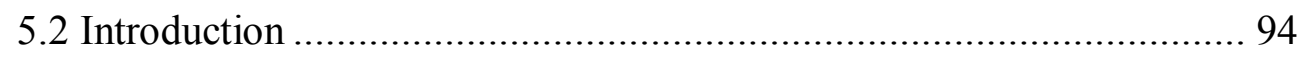

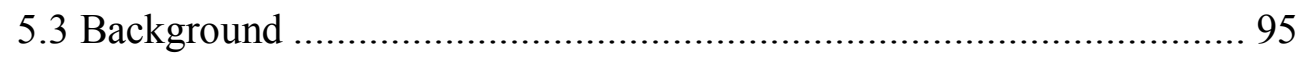

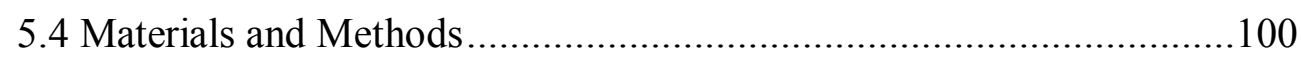

5.4.1 Experimental Set-Up .........................................................100

5.4.2 Creation of the Scaling Factor .................................................104

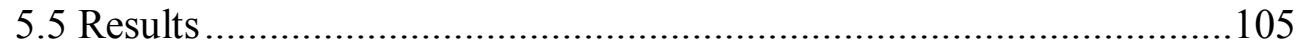

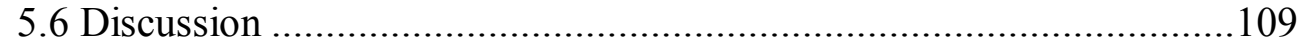

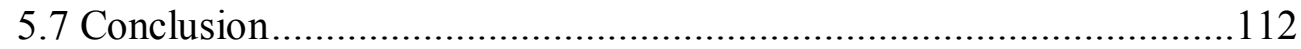

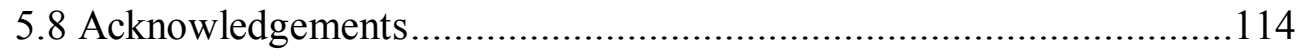

$\begin{array}{lr}\text { CHAPTER } 6 & 115\end{array}$

FIRST-ORDER THERMAL LENSING MODELING PREDICTIONS OF RETINAL

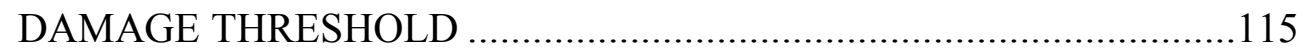

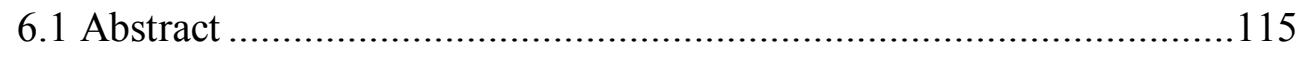

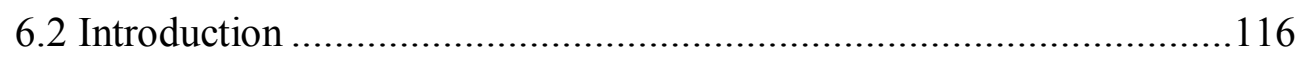

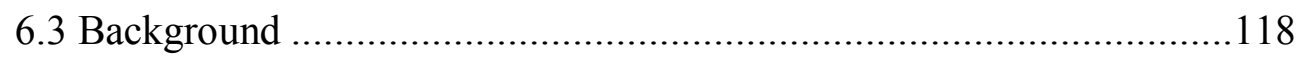

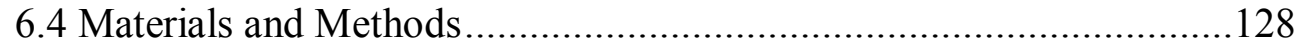

6.4.1 Selection of $\mu_{\mathrm{a}}$ and Predicting Damage Thresholds as a Function of Input Beam Diameter ...........................................................128

6.4.2 Comparing First-Order Model to BTEC Model Results ...........131 
6.5 Results..... 133

6.5.1 Predicting Damage Threshold as a Function of Input Beam Diameter Using the First-Order Thermal Lensing Model 133

6.5.2 BTEC vs. First-Order Thermal Lensing Model .........................140

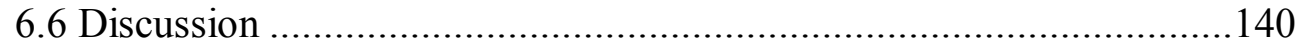

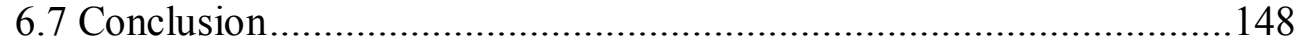

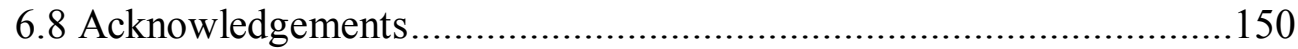

CHAPTER 7

OBSERVATIONAL METHOD FOR QUANTIFYING THERMAL LENSING

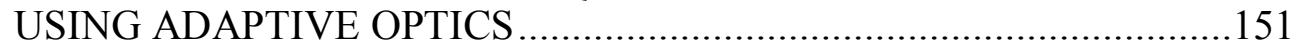

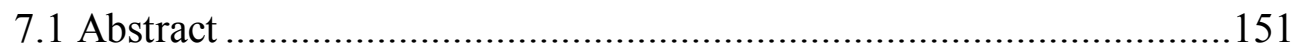

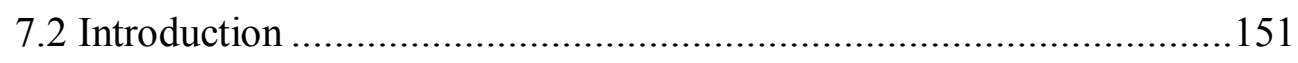

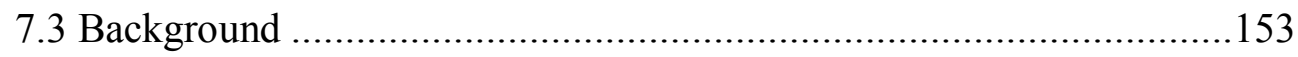

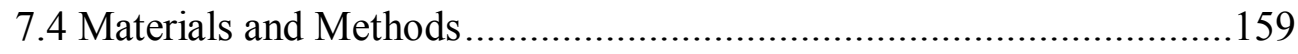

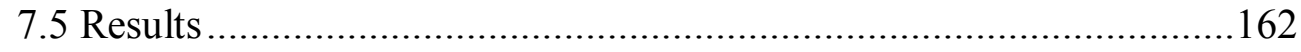

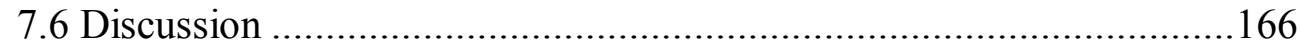

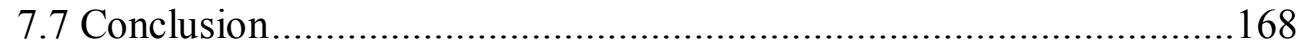

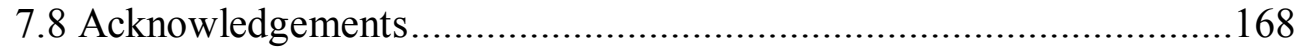

$\begin{array}{lr}\text { CHAPTER } 8 & 169\end{array}$

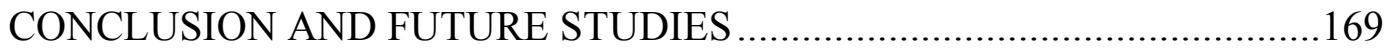

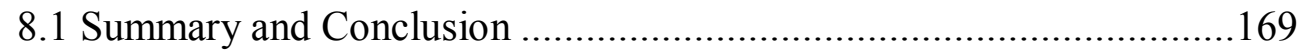

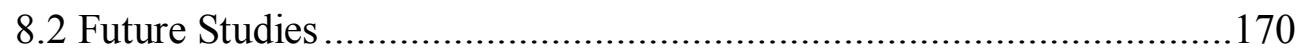

8.2.1 Retinal $\mathrm{ED}_{50}$ as a Function of Delivered Corneal Spot-size .....170

8.2.2 Quantifying Aberrations Induced by Thermal Lensing in vivo 171

8.2.3 Histology and Advanced Imaging...........................................171

8.2.4 Advanced Retinal Damage Modeling .....................................172

8.2.5 Measuring Thermal and Optical Properties of Tissues .............173

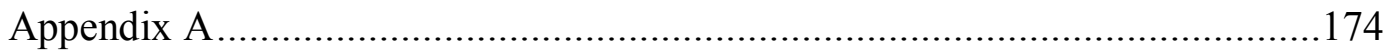

Documentation of Code for Chapter 4..................................................174 


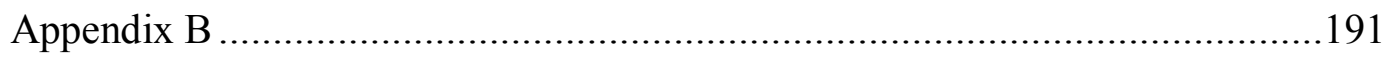

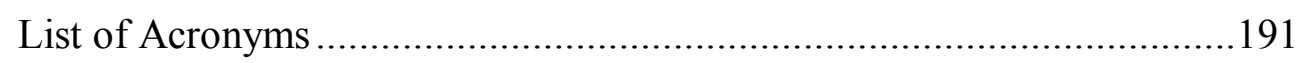

Bibliography ................................................................................. 192

Vita 202 


\section{List of Tables}

Table 2.1: $\mathrm{ED}_{50}$ (reported for retina as a TIE) values for retina from 1315-1330 nm. The TIE in $\mathrm{J}$ is the reported $\mathrm{ED}_{50}$ measurement from literature. The TIE in $\mathrm{W} / \mathrm{cm}^{2}$ was calculated based on

TIE(J)/(Pulsewidth(sec)*Approximate area at cornea $\left.\left(\mathrm{cm}^{2}\right)\right)$. The percent transmission was calculated from data reported by Maher 1978 and can be found in section 2.2.2. 13

Table 2.2: $\mathrm{ED}_{50}$ values for cornea from $1315-1356 \mathrm{~nm}$. 14

Table 2.3: Linear absorption coefficient, $\mu_{\mathrm{a}}\left(\mathrm{cm}^{-1}\right)$, in the $1300-1330 \mathrm{~nm}$ region for ocular tissues interpolated from the data of Maher 1978 24

Table 2.4: Percent of light transmission to RPE for rabbit, rhesus and human eyes based on results from Fig. 2.6. 25

Table 2.5: Sellmeier coefficients found to produce a fit to the iterative solution for $n(\lambda)$, see Fig. 2.7b, based upon the refractive error values of the human eye from Liou and Brennan 1997, and Fernandez, Unterhuber et al. 2005, in Fig. 2.7a. 29

Table 3.1: A summary of the experimental data matrix for the optical configuration depicted in Fig. 3.4. $\Delta \mathrm{z}$ describes the position of the confocal system's imaging plane relative to the initial-state focus at $\mathrm{t}=0 \mathrm{with}$ negative and positive values denoting imaging planes in front of and behind the initial-state focus respectively. Power is reported as the power reaching the front surface of the quartz-cuvette. 
Table 3.2: A summary of the experimental data matrices for the optical configuration in Fig. 3.5. Power is reported as the power reaching the front surface of the Cain-cell lens. Based on results from Fig. 3.1b, the power reaching the focal position for the NIR laser radiation is approximately 54.2, 39.0, 27.5 , and $8.1 \%$ of the power input at the front of the Cain cell lens for $1110,1130,1150$ and 1318-nm wavelengths respectively (the percentages do account for an approximate $12 \%$ loss in the lens: loss based upon measurements and simulations for an empty and water-filled cell) 48

Table 3.3: The coefficients for the fits provided in Fig. 3.10. The coefficients A, B, $\mathrm{E}$ and $\mathrm{F}$ are described in Eq. 3.2 and 3.3. The logarithmic and exponential regression fit yielded $\mathrm{R}^{2}$ (the statistical correlation coefficient) values $>0.9$ for all data sets. 58

Table 3.4: The values for slope, $m$, from the linear fits depicted in Fig. 3.11 fitting the generic form in Eq. 3.4 at time, t, during the $\mathrm{CW}$ exposure. ..... 59

Table 4.1: Thermal conductivity, $\kappa$, density, $\rho$, specific heat at constant pressure, $\mathrm{c}_{\mathrm{p}}$, and thermal diffusivity, $\eta$, as a function of temperature, $T$, found to fit data for liquid water from Wagner et al. for $0.1 \mathrm{MPa}$ between 283.15 to 363.15 K (Wagner and Kruse 1998). 77

Table 4.2: Linear absorption coefficients for ocular media and water at 1150 and 1318 nm (Hale and Querry 1973; Maher 1978). 78

Table 5.1: The linear absorption coefficient of the RPE, $\mu_{\mathrm{a}}$, for the laser radiation wavelengths in this study. 98 
Table 5.2: The parameter sets for CW NIR exposures investigated in this study. $\mathrm{P}$ and $\mathrm{M}$ are for paramacula and macula respectively. A Mira-OPO laser was used for the 1110,1130 and 1150-nm wavelength $(\lambda)$ studies while a Lee laser was used for the 1319-nm study. The OPO and Lee laser sources had a $\theta$, full-angle beam divergence, of 1.5 and $13.3 \mathrm{mrad}$ respectively. 102

Table 5.3: Summary of the data obtained from the retinal ( $\mathrm{P}$ for paramacula, $\mathrm{M}$ for macula) damage thresholds. The slopes of all Probit results were greater than 2, save for the $1150-\mathrm{nm}$ radiation cases for subjects 2,3 and combined $1,2 \& 3$ where $\mathrm{N} / \mathrm{A}$ is entered. N/A entered for an $\mathrm{ED}_{50}$ indicates no lesions were observed, even though exposures were at 830-mW (maximum power available for 1150-nm) for 100-ms. N/A entered in a fiducial limit (FL) indicates the FL was unattainable for the relevant data set............................................................... 106

Table 5.4: The scaling factor, SF, from Eq. 5.2, for several wavelengths in the NIR. The SF is intended to be multiplied by the $1110-\mathrm{nm}$ laser radiation wavelength's 24-Hr $\mathrm{ED}_{50}$. .107

Table 6.1: The thermal coefficients of the simple rhesus eye needed for the thermal lensing model. The terms $\kappa, \rho$ and $c_{p}$ are the thermal conductivity, density and specific heat at constant pressure respectively. Values for the cornea, aqueous, lens and vitreous were obtained from Okonu, Kojima et al. 2005, and values for water were from Wagner and Kruse 1998. All parts of the retina were assumed to have the same thermal properties as the vitreous 123 
Table 6.2: The optical coefficients of the simple rhesus eye model. The terms $n_{0}$ and $\mu_{\mathrm{a}}$ are the background index of refraction (all layers of the eye are treated as index matched) and the linear absorption coefficient respectively. The values for $\mathrm{n}_{0}$ were determined previously in Chapter 2 and Vincelette, Rockwell et al. 2008. The values for $\mu_{\mathrm{a}}$ were from Maher 1978, Hale and Querry 1973, and by fitting a curve to data from Birngruber, Hillenkamp et al. 1985. (C - cornea, A - aqueous, L - lens, $\mathrm{V}$ - vitreous, $\mathrm{W}$ - water, $\mathrm{PE}$ - pigment epithelium). Note the PE values reported here were not used for this study; they are presented merely for comparison and assumed to include water. 124

Table 6.3: The increase from initial beam diameter at the retinal plane for the retinal $\mathrm{ED}_{50}$ 's of 1319,1150 and 1110 -nm wavelengths for varying input beam diameters at the corneal plane. These results were tabulated using the mathematical model presented in Chapter 4. The model's input parameters for power, exposure duration and wavelength were selected from the experimental results for the $\mathrm{ED}_{50}$ values from Chapter 5.125

Table 6.4: The 24-hour $\mathrm{ED}_{50}$ from Chapter 5. ${ }^{1}$ Indicates set was determined experimentally. ${ }^{2}$ Indicates the set was estimated from the SF method. .126

Table 6.5: The parameter sets which were simulated in this study. The temperatures of each set were calculated. The time to reach the damage threshold temperature in each set was recorded for conditions with and without thermal lensing. * - Denotes the input parameter set used to establish the damage threshold temperature for the respective wavelength........131 
Table 6.6: The values for $\mu_{\mathrm{a}}$ used for the homogenous retinal slab, assumed to have equal water and pigment distributed uniformly throughout, which gave the computational upper and lower limit of $\Delta \mathrm{T}\left(50^{\circ} \mathrm{C}\right.$ and $\left.13^{\circ} \mathrm{C}\right)$. All cases were computed with thermal lensing on in the first-order model. Unless stated otherwise, the $\mu_{\mathrm{a}}$ values for the upper limit were used for the remainder of the computations in this chapter. The $\mu_{\mathrm{a}}$ values for water are given for comparison and were obtained from Hale and Querry 1973. Input parameters for power and beam-diameter at each wavelength were given in Table 6.4. 134

Table 6.7: The side-by-side results from the first-order thermal lensing (FOTL) and BTEC models with and without axial conduction. The temperature rise is taken at the observational time point for a $\mathrm{CW}$ laser exposure (the laser source never turns off). The simulated eye in these computations used a homogenous $\mu_{\mathrm{a}}=1.3598 \mathrm{~cm}^{-1}$ (the value for water) and compartmentalized thermal coefficients from Table 6.1.

Table 7.1: The aberration types representing the Zernike polynomial coefficients (beginning with 0) listed in their order of appearance in the Iris AO system's algorithm. 158 


\section{List of Figures}

Figure 2.1: Fundus image of a rabbit subject (Zuclich, Schuschereba et al. 1997).

The arrows indicate the 514-nm marker lesions. Lesions adjacent to the marker lesions are from CW (exposure lengths of $1 \mathrm{~s}-10 \mathrm{~s}$ ) 1318-nm NIR exposures as they appeared 24-hours post-exposure. Zuclich et al. estimated that the argon lesions measured $200 \mu \mathrm{m}$ in diameter. This image first appeared in Zuclich, Schuschereba et al. "A comparison of laser induced retinal damage from infrared wavelengths to that from visible wavelengths." Lasers and Light in Ophthalmology. 8(1). p. 19 (1997). 16

Figure 2.2: The total action spectrum for the rhesus eye for ns and 0.1-s exposures compared to corresponding experimental retinal $\mathrm{ED}_{50}$ data thresholds from 400-1350-nm wavelengths. Re-printed with permission from Zuclich, Lund et al. "Wavelength dependence of ocular damage thresholds in the near-IR to far-IR transition region: Proposed revisions to MPEs.” Health Physics. 92(1), p. 17. (2007)........................... 18

Figure 2.3: A fit to the absorption data of rhesus PE from Birngruber, Hillenkamp et al.1985 data described by Eq. 2.1 21

Figure 2.4: Physiological data for eyes of three species represented as comparable schematic eyes (Hughes 1972; Westheimer 1980; Li, Zwick et al. 2000; Fernandes, Bradley et al. 2003). Axial length is the sum of the distances from the front of the cornea at position a to the front of the retina at position e. Note the human lens thickness data includes the cortex, core and post-cortex for a total lens. 23

$$
\text { xix }
$$


Figure 2.5: Linear absorption coefficient, $\mu_{\mathrm{a}}$, as a function of wavelength for ocular media from rhesus eyes and water (Hale and Querry 1973; Maher 1978).

Figure 2.6: Percent of transmitted light reaching the retina in the schematic eye from Figure 2.4. Transmission values were calculated by Beer's Law using ocular absorption values from Fig. 2.5 for respective ocular components. Calculations used here did not account for specular reflections at any boundary. 25

Figure 2.7: (a) Compilation of refractive error of the human eye for several summary reports including an estimate of refractive error for computing the results by Lund, Edsall et al. 2000 (Liou and Brennan 1997; Lund and Edsall 1999; Atchison and Smith 2005; Fernández, Unterhuber et al. 2005). The linear fit from the Fernandez, Unterhuber et al. 2005 data was shifted to match the refractive error of the Liou and Brennan 1997, modeled at $700 \mathrm{~nm}$. (b) Result for $\mathrm{n}(\lambda)$ based on the Liou and Brennan 1997, and Fernandez, Unterbuber, et al. 2005 data from (a). The Sellmeier fit to produce this result used the coefficients given in Table 2.5 . 29

Figure 2.8: Predicted spot size at a virtual retinal plane using the information from the reduced eye model in conjunction with the dispersion data from Fig. $2.7 \mathrm{~b}$ 30 
Figure 2.9: Changes in a minimum spot formed by a 25.4- $\mathrm{mm}$ focal length lens for a 633-nm beam co-aligned with a $1313-\mathrm{nm}$ beam passing through a $1-\mathrm{cm}$ thick sample of vitreous $\left(\mu_{\mathrm{a}}=1.77 \mathrm{~cm}^{-1}\right)$ at $0,20,47$ and $127 \mathrm{~ms}$. The spot begins to diverge, or bloom, becoming non-linear after a period of time in the exposure depending on absorptive properties of the sample and input power (Vincelette, Thomas et al. 2007)...................... 32

Figure 3.1: (a) The linear absorption coefficients for various media used to predict the percent transmission (Hale and Querry 1973; Maher 1978). (b) The percent of transmitted energy from the surface of the cornea to the retina for a rhesus eye (assuming no specular reflection) and the water-filled Cain cell (assuming 12\% loss in energy from the Cain-cell lens) (Vincelette, Rockwell et al. 2008). The average loss in the Cain cell lens was determined experimentally over a range of near-infrared wavelengths (see methods section).

Figure 3.2: The optical configuration of the Cain-cell artificial eye (Cain, Noojin et al. 1997). The custom lens is made from SF11 glass. To create the curve in Fig. 3.1b for the Cain cell, 100\% energy was assumed at the surface of the lens, and a $\sim 12 \%$ loss was assumed through the lens (loss was determined using a Coherent power-meter in an empty Cain-cell and Zemax simulations). Finally Beer's law was applied to the waterfilled chamber to determine the transmitted energy at the focus..... 38 
Figure 3.3: (a) The confocal imaging system at observational imaging planes before, at and after the probe-beam's focus, $z=0$. AE is the artificial eye (composed of a short focal length lens and a sample holding chamber). Obj. is the microscope objective. Cam. is the camera. (b) The resulting transient information provided by exposures at each of the respective imaging planes in (a)

Figure 3.4: The optical set up using the modified artificial eye. M1-3: Broadbandvisible mirrors. M4 and M5: Near-infrared mirrors. L1-5: Thorlabs lenses with -100, 500, -100, 200 and 25.4-mm focal lengths respectively. SW: 10-mm diameter electronic shutter. DM: Dichroic mirror made (CVI LWP-0-R633-T1319-PW-1025-C) transmitted the pump and reflected the probe laser. QC: Quartz cuvette to hold the sample. The imaging system consisted of a Mitutoyo objective (Obj.), a Newport optics 200-mm lens (L6), neutral density filters (ND) and a Photon Focus camera (cam., model MV-D1024-160-CL-8). Delay generator: Stanford Research Systems 4-Channel Digital Delay Generator, model DG-535. Shutter controller: Uniblitz SD-10 Shutter Driver Timer. Pulse generator: HP8116A Pulse/Function Generator. The National Instruments frame-grabbing card was a NI PCI-e1427 and installed on a Dell Dimension E521 (Model DC6M) with an AMD 64 Athlon X2 Processor running Windows XP. The imaging system is moved along the optical axis to observe individual positions in $\mathrm{z}$-space relative to the back of the sample cuvette through time at a high frame-rate. 44 
Figure 3.5: The experimental set up using Cain-cell artificial eye. L1-3 were -10, 50 and $20 \mathrm{~cm}$ focal length Thorlab BK7 glass lenses respectively. M1-3 were broad-band visible mirrors while M4 \& 5 were silver coated mirrors. WP was the waveplate to control the power. SW was the Uniblitz electronic shutter. DM was a dichroic mirror to transmit in the NIR and reflect in the visible to co-alignment the beams. AE was the artificial eye. IRF was an infrared filter to protect the camera from the high-power NIR exposure. The long working distance $(3.3 \mathrm{~cm})$ microscope objective (Obj.), lens element L6 and camera (Cam.) comprised the confocal imaging system and were mounted together on a micrometer stage. ND was the neutral density filter to control saturation of the time $=0$ image at $\mathrm{z}=0$. 46

Figure 3.6: Some examples of the resulting video-frames compiled from the data analysis of vitreous and water samples in the modified artificial eye at (a) resting/initial-state, (b) at steady-state (time $=1 \mathrm{~s}),(\mathrm{c})$ at $0.16 \mathrm{~s}$ after the pump-laser is turned off and (d) $1 \mathrm{~s}$ after the pump-laser is turned off. 52

Figure 3.7: Comparison of the rates of the thermal lens induced in the vitreous and water samples exposed to $129-\mathrm{mW}$ of $\mathrm{CW}$ laser-radiation at a wavelength of 1313-nm for 1-s. Experimental data presented in this figure are the result of analyzing the video results as given in the example frames in Fig 3.6. $\Delta \mathrm{z}$ represents the focal plane of the confocal imaging system relative to the initial-state focus found at time $=0$. Error bars were previously explained in the text. 53 
Figure 3.8: Comparison of the rates of the thermal lens induced in the vitreous sample exposed to two different power levels of $\mathrm{CW}$ laser-radiation at $1313-\mathrm{nm}$ for $1 \mathrm{~s}$ 53

Figure 3.9: An example of the video frames which were compiled for analyzing the data sets for all four laser-radiation wavelengths at time, $\mathrm{t}=0$ and $1 \mathrm{~s}$ of the 1-s excitation exposure to $150-\mathrm{mW}$ of power input at the Cain-cell lens. (a) The initial state and (b) shows the steady-state. The value of the z-labels identifies where the confocal imaging system was placed relative to the probe-beam's initial focus at $\mathrm{t}=0$. The error associated with the time slice is $+/-0.00667 \mathrm{~s}$. Differences in the thermal lensing effect between wavelengths are reserved for the discussion. 55

Figure 3.10: The excitation and relaxation of the thermal lens from the pump-laser radiation wavelengths of (a) 1110, (b) 1130, (c) 1150 and (d) $1318 \mathrm{~nm}$.

Figure 3.11: The relationship between refractive error and power for each wavelength evaluated at (a) 100-ms and (b) 250-ms exposures. The fits to data were generated by a linear fitting algorithm with a forced zerointercept in Matlab. 59

Figure 4.1: An example of a negative thermal lens z-scan. The case for the sample exposed at z-positions (a) very far from the focus of the lens and very near or at the focus resulting in unity normalized irradiance, (b) before the focus resulting in an increase in normalized signal irradiance, and (c) after the focus resulting in a decrease in signal irradiance. (d) The resulting $\mathrm{z}$-scan data set 69 
Figure 4.2: The closed-aperture, single-beam z-scan set up. The z-axis is referenced to the focal position of the lens found using a knife-edge technique in air, with no cuvette in the beam's path. The laser was a 1313-nm CrystaLaser IRCL-150-1313-P-L. M1-M4: IR-coated mirrors. L1 and L2: IR-coated lenses created a beam expander of $\sim 1.5 x$. L3: 400-mm ThorLabs plano-convex 1-in lens. SW: 1-cm diameter Uniblitz shutter. Quartz Cuvette: from Starna Cells. A: Aperture set to 2.5-mm diameter. D: 818-IR Newport detector. 75

Figure 4.3: The simple eye model with a radius of curvature of $0.61 \mathrm{~cm}$ on the front surface of the cornea brings a collimated, 589-nm beam to a minimum at the virtual retinal plane $2.44 \mathrm{~cm}$ after the front surface of the eye. The vitreous layer is infinitely long, with beam trend observations made at the position of the virtual retina. 78

Figure 4.4: Values of $\mathrm{dn} / \mathrm{dT}(1 / \mathrm{K})$ as a function of temperature for water in the liquid phase for five different wavelengths. Values here were based upon taking the derivative of $n(T)_{P}$ at each of the five wavelengths as reported in literature at 0.1 MPa (Schiebener, Straub et al. 1990; Harvey, Gallagher et al. 1998). 80

Figure 4.5: Z-scan data for a 10-mm water-filled cuvette exposed to $1313-\mathrm{nm} \mathrm{CW}$ laser source for $1 \mathrm{~s}$ at (a) $2.6 \mathrm{~mW}$ and (b) $48 \mathrm{~mW}$...... 81

Figure 4.6: The transient response for the z-scan peaks from Fig. 4.5 for $2.6 \mathrm{~mW}$ (a) maximum and (b) minimum and $48 \mathrm{~mW}$ (c) maximum and (d) minimum 
Figure 4.7: Increase in the beam radius at the retina as a function of time for selected powers delivered to the cornea. Parameters were for 1318-nm, CW laser radiation into a human eye, with a $1 / \mathrm{e}^{2}$ Gaussian beam radius of 2.12 $\mathrm{mm}$ at the virtual cornea. The $\mathrm{y}$-axis was normalized to $98 \mu \mathrm{m}$, which was the beam radius at the virtual retina determined at time $=0 \mathrm{~s} . .83$

Figure 4.8: Increase in the beam radius at the retina as a function of time for selected input beam radii at the cornea. 84

Figure 4.9: Increase in the beam-radius for 1318-nm laser radiation at the retina as a function of input power at the cornea for (a) short time ( $\mathrm{t} \leq 1 \mathrm{~ms})$ and (b) long time $(1 \mathrm{~ms}<\mathrm{t} \leq 250 \mathrm{~ms})$. Note the $340-\mathrm{W}$ power at $0.350 \mathrm{~ms}$ is approximating the $\mathrm{ED}_{50}$ threshold level reported in Zuclich, Lund et al. 2004, for the equivalent wavelength. The y-axis was normalized to the initial beam radius of $98 \mu \mathrm{m}$. 85

Figure 4.10: Increase in the beam-radius for 1150-nm laser-radiation at the retina as a function of input power at the cornea for (a) short time ( $\mathrm{t} \leq 1 \mathrm{~ms})$ and (b) long time $(1 \mathrm{~ms}<\mathrm{t} \leq 250 \mathrm{~ms})$. The $\mathrm{y}$-axis was normalized to the initial beam radius of $82 \mu \mathrm{m}$. 85

Figure 4.11: Increase in the beam-radius for 1318-nm laser radiation at the retina as a function of input power at the cornea for (a) short time ( $\mathrm{t} \leq 1 \mathrm{~ms})$ and (b) long time ( $1 \mathrm{~ms}<\mathrm{t} \leq 250 \mathrm{~ms})$ having doubled the value of $\mathrm{dn} / \mathrm{dT}$ to $8.92 * 10^{-5}\left(\mathrm{~K}^{-1}\right)$. The y-axis was normalized to the initial beam radius of $98 \mu \mathrm{m}$. 86 
Figure 5.1: Transmission of laser radiation in a human and rhesus eye based on linear absorption data of Maher 1978. These results assumed physical thicknesses of the cornea, aqueous humor, crystalline lens and vitreous humor from literature (Westheimer 1980; Li, Zwick et al. 2000; Fernandes, Bradley et al. 2003) 97

Figure 5.2: Absorption by retinal pigment epithelium, RPE, based on a fit to data from Birngruber, Hillenkamp et al. 1985 98

Figure 5.3: The 1/ $\mathrm{e}^{2}$ laser-spot diameter for NIR laser radiation wavelengths delivered to the rhesus retina with chromatic dispersion. 99

Figure 5.4: The optical set up. L1, L2 - lenses formed a 5x beam expander for the 633-nm pointing laser. M1-5 - broad-band visible mirrors. M6-8 silver mirrors. M7 and M8 formed a periscope to bring the beam up vertically to the viewing level of the fundus camera. BS1 - a beam splitter which combined the two visible lasers. BS2 and BS3-identical beam splitters used to combine all three wavelengths. WP - waveplate to control power level sent down the optical train. S1 and S2 were two independently controllable electronic shutters set for exposure durations of $\sim 25$ and $100 \mathrm{~ms}$ respectively. P1 - reference power meter to determine the power to be delivered to the corneal plane. P2 photodiode connected to an oscilloscope to verify the exposure duration of the laser-radiation. NIR Cam. - near-infrared camera used to ensure no clipping of the pupil was occurring during laser exposure 
Figure 5.5: The 24-Hr, 100-ms, ED E0 retinal damage thresholds from Table 5.3 (1319-nm data were scaled to 100-ms by Eq. 5.4) and the SF fit determined by mulitplying the SF values (Table 5.4) by the $1110-\mathrm{nm}$ $\mathrm{ED}_{50}$ threshold for combined subjects. 107

Figure 5.6: Fundus camera images captured of subject 1 at 24-Hr post-exposure to 1150-nm laser radiation (field of view was $35^{\circ}$ for both images). The letter $\mathrm{M}$ denotes a marker lesion, with $\mathrm{M}_{0}$ acting as a reference marker between $a$ and $b$. The numbers marked below the grid locations represent the power, in $\mathrm{mW}$, delivered to the corneal plane for 100-ms. Lesions without numbers are marker lesions used to reference the grid location of each NIR radiation exposure on the fundus. (a) Displays the large lesions observed for the first 1150-nm exposure with a resulting $\mathrm{ED}_{50}$ of $406 \mathrm{~mW}$. (b) Displays lesions observed for the second 1150-nm exposure, conducted 3-weeks later, on the same eye resulting in an $\mathrm{ED}_{50}$ of $567 \mathrm{~mW}$. The arrows point to exposure areas off the marker grid in attempts to check experimental repeatability. Combining all yes/no data for both exposure sessions (data from both $a$ and $b$ ) gave the resulting $\mathrm{ED}_{50}$ of $439 \mathrm{~mW}$ reported in Table 5.3 108

Figure 6.1: The simple eye model for a rhesus subject. The radius of curvature, $r_{c}$, on the cornea is $0.498 \mathrm{~cm}$, in order to achieve a minimum beam-waist for a collimated beam of 589-nm laser radiation at the front surface of the retina. The front-surface of the retina layer was placed along the $\mathrm{z}$-axis at $1.99 \mathrm{~cm}$ after the corneal surface. .123 
Figure 6.2: The beam-waist radius as a function of time for a collimated (a) 5-mm and (b) 3-mm 1/ $\mathrm{e}^{2}$ diameter delivered to the corneal plane. These data were used as inputs into the computations to determine the source term and temperature rise as the retina. (Note that this step does not require $\mu_{\mathrm{a}}$ of the retina.) 135

Figure 6.3: The source terms generated at the retina at $r=0$, using the $\omega(\mathrm{z}=$ retina, $\mathrm{t})$ from Fig. $6.2\left(\mu_{\mathrm{a}}\right.$ values given in Table 6.6 for $\Delta \mathrm{T}_{0}$ of $\left.50^{\circ} \mathrm{C}\right)$. 136

Figure 6.4: The result of Eq. 6.1 for the two specified cases with and without thermal lensing for the collimated $1 / \mathrm{e}^{2}$ input beam diameters of (a) 5-mm and (b) 3-mm. 136

Figure 6.5: The resulting $\Delta \mathrm{T}$ at the anterior retinal surface at the $\mathrm{r}=0$ position as a function of $t$ for the two specified cases with and without thermal lensing (assuming no axial diffusion). The threshold temperature, $\Delta \mathrm{T}_{0}$, was selected to be $50^{\circ} \mathrm{C}$. The (a) $5-\mathrm{mm}$ case shows some variation in the temperature rise between the cases with and without thermal lensing. The (b) 3-mm case shows a substantial difference in the temperature rise during an 80-ms exposure. 137

Figure 6.6: The predicted $\mathrm{ED}_{50}$ as a function of the input beam-diameter at the corneal plane. Values were determined by when the condition was able to reach $\Delta \mathrm{T}_{0}$ of $50^{\circ} \mathrm{C}$.

xxix 
Figure 7.1: (a) A perfect wavefront passes through the lenslet array in a ShackHartmann system. The result is an ideal configuration of spots on the CCD sensor. (b) An imperfect wavefront passes through the lenslet array. Notice the slope of the cone angles depicted in a and $b$; the location of the spot on the CCD grid helps to determine the incoming angle (translated into a slope) of the beam-segment from the distorted wavefront. These slopes are used to reconstruct the wavefront at the image plane. The segmented wavefront represents how each lens in the array takes in only a small segment of the wavefront and brings that portion of the wavefront to focus at some position on the CCD sensor. The position where the wave segment is focused on the CCD is relative to the slope the light segment forms as it exits the individual lens. 154

Figure 7.2: Basic optical configuration of a Shack-Hartmann system to measure the wavefront aberrations associated with the eye (Thibos 2000). ......155

Figure 7.3: A typical wave-front feedback loop for an adaptive optics (AO) ocular imaging system. (DM - Deformable Mirror, BS - Beam Splitter, WFS Wavefront Sensor). Currently, AO feedback loops update the image and DM position at a rate from $15-30 \mathrm{~Hz}$.....................................156

Figure 7.4: The periodic table of Zernike polynomials represented as respective wavefront shapes. See Table 7.1 for the type of aberration corresponding with each mode. Figure from Thibos 2001...........157 
Figure 7.5: The reconstructed wavefront for the Cain-cell sample with ( $\mathrm{a} \& \mathrm{~b})$ the wavefront corrected, locked in place and no thermal lensing, and (c \& d) the aberrations resulting purely from the induced thermal lens (shown after the 1150-nm laser has been continuously exposed to the sample for $\sim 5.3 \mathrm{~s}$. The laser was blocked after $8.25 \mathrm{~s}$ ) 162

Figure 7.6: The corresponding point-spread function (PSF) for the reconstructed wavefronts depicted in Fig. 7.5. In (a) - the initial-state PSF, (b) - the steady-state, thermal lens induced PSF. 163

Figure 7.7: The uncorrected (flat DM) wavefront recorded from the Cain-cell lens in (a) 2D and (b) 3D, without thermal lensing (the NIR beam was blocked). 164

Figure 7.8: The PSF corresponding to the wavefront in Fig. 7.7. 164

Figure 7.9: The transient response to the thermal lens for five Zernike polynomial coefficients. The 1150-nm exposures were approximately 7.5 -s and 8.25-s for the first and second exposures respectively 165 


\section{CHAPTER 1}

\section{INTRODUCTION}

\subsection{Motivation}

The United States military has been investigating the use of high energy lasers operating in the near-infrared for several decades (Lange, Pitz et al. 2005). The safety parameters which must be in place before users are exposed to these new technologies are a responsibility of the Department of Defense, DoD. Formulation of safety standards includes modeling scenarios for exposure and performing in vivo experiments to assess risk of exposures. Damage threshold studies for the skin, cornea and retina, are essential to validate models and range surveys to have any quantitative meaning. The knowledge gained from experimental data and modeling simulations of laser-tissue interaction is essential for providing the DoD with appropriate safety margins needed for operations involving laser devices. Given the importance of the eye, laser safety research is directed to evaluate hazards to the retina.

Existing laser safety data spanning a wide range of wavelengths over several decades can be found in the literature. Much of these data are reflected in exposure standards set by the American National Standards Institute (ANSI) in the Z136 series which provides guidelines on safe levels of exposure for various tissues and wavelengths and in the international community by the International Commission of Non-Ionizing Radiation Protection (ICNIRP) (ANSI 2000; ICNIRP 2000). For studies on retinal

damage thresholds, parameters such as beam size at the cornea, beam divergence, pupil diameter, exposure duration, pulse repetition rate and multiple wavelength exposures are 
based upon existing threshold data. Unfortunately, these parameters are not well characterized for regions in the near infrared where water absorption becomes significant (van den Berg and Spekreijse 1997). Accurate damage predictions in this region require investigational studies to evaluate ocular damage thresholds where a multitude of parameters are considered. Threshold damage from near-infrared laser radiation is typically thermal in nature for those wavelengths that reach the retina.

The near infrared, NIR, region from 1150 to $1350 \mathrm{~nm}$ is unique in terms of potential hazards to the cornea and retina. Wavelengths in this NIR region have 1/e penetration depths on the order of $700 \mu \mathrm{m}$; long enough to easily pass through the cornea, but short enough that one to ten percent of the total intraocular energy reaches the retina (Boettner and Wolter 1962; Maher 1978; van den Berg and Spekreijse 1997). At these wavelengths, little change in the linear absorption coefficient, $\mu_{\mathrm{a}}$, occurs with increasing temperature (Walsh 1995). Nevertheless, there should be sufficient energy to ensure retinal lesions for a collimated beam entering the eye without damage to the cornea. Experimental safety studies in the $1300 \mathrm{~nm}$ regime report retinal damage thresholds for single-pulse exposures of $50 \mathrm{~ns}-650 \mu \mathrm{s}$ in rhesus subjects, while continuous wave, $\mathrm{CW}$, exposures of 0.1-10 s can produce corneal lesions, but never retinal damage (Stuck, Lund et al. 1980; Lund, Stuck et al. 1981; Zuclich, Gagliano et al. 1995; Lund, Edsall et al. 1998; Zuclich, Lund et al. 2001; Zuclich, Lund et al. 2004; Zuclich, Lund et al. 2007). However, the same experiments performed on rabbits cause retinal damage. This anomaly of producing CW retinal damage lesions between species has only been observed in this particular wavelength region. The important wavelength dependent factors effecting retinal threshold damage are (i) the percent of laser light reaching the retina, (ii) the spot size of the laser beam at the retina and (iii) the relative absorption by 
the retinal pigment epithelium, RPE, and water in the retina. In many theoretical studies, the laser-beam's spot size delivered to the retina is taken to be a static variable, subject to chromatic dispersion, but independent of time or thermal effects. I hypothesize that thermal lensing induces a transient response in the beam-waist radius delivered to the retina.

Thermal lensing, also known as thermal blooming, is a well known phenomenon discovered in 1965 by Gordon et al. (Gordon, Leite et al. 1965). Thermal lensing results from a radial temperature gradient caused by the absorption of laser radiation along the beam path. Radial temperature gradients, and to a lesser degree, axial absorption causes local changes in the index of refraction. As the temperature, $\mathrm{T}$, in an aqueous sample increases, density, $\rho$ (mass/volume), and the index of refraction, $n$, both decrease. Thus, for ocular media exposed to a continuous-wave (CW) laser-radiation source with a Gaussian-beam profile, the temperature rise is largest at the center of the beam where the radial distance, $r=0$. The index of refraction, $n$, along the radial distance, $r$, changes from the baseline index of refraction, $\mathrm{n}_{0}$, at a maximum along the center of the beam $(\mathrm{n}<$ $\mathrm{n}_{0}$ at $\mathrm{r}=0$ for time $\left.>0\right)$ and minimum at the edge of the beam-profile $\left(\mathrm{n}=\mathrm{n}_{0}\right.$ at $\mathrm{r}>>0$ for time $>0)$. This causes the index of refraction, $n(r, z, t)$, along the beam path to become spatially dependent upon the temperature distribution in the media with respect to radius, $\mathrm{r}$, and the optical axis, $\mathrm{z}$, and time, $\mathrm{t}$. The temperature distribution in the radial direction causes the formation of a graded negative lens for the laser beam propagating through the ocular media, thus dynamically changing the size of the beam-waist delivered to the retina. Any change in the spot size at the retina directly impacts the local rate of heat generation. Understanding the role of thermal lensing in ocular damage mechanisms will 
allow for more accurate predictions of damage thresholds to establish a quantitative link between safety and efficacy for laser technologies.

This research investigated the thermal lensing effect in aqueous media to quantify the dynamic changes in ocular index of refraction for near-infrared laser radiation wavelengths from 1110-1319-nm. The investigations included modeling and measurements of the optical-thermal response in an artificial eye. The research also included a CW retinal exposure study which determined the damage threshold over these wavelengths and linked the thermal lensing effect to these data. Experiments were conducted in an artificial eye varying wavelength, time of exposure, and radiant power at the cornea to provide a quantitative multivariate analysis of thermal lensing.

\subsection{DisSERTATION OVERVIEW AND SPECIFIC AIMS}

This dissertation consists of three primary components; the first being damage threshold studies, the second describes and evaluates mathematical models and the third provides physical observations pertaining to the thermal lens. The layout of the chapters follows the scientific method. Chapters 2-7 address the specific aims detailed below. Chapter 8 summarizes the findings of the dissertation and the role of thermal lensing in the eye in relation to retinal damage thresholds.

The ultimate goal of my research was to understand the role thermal lensing had in the formation of a lesion from a continuous-wave, CW, near-infrared, NIR, laser radiation at the retina and the irradiation parameters required to form the retinal lesion. This research investigated wavelengths in the $1100-1350 \mathrm{~nm}$ region. To achieve this goal, the following six specific aims were explored: 
Specific Aim 1: Specify the scope of the problem with the NIR retinal lesion studies in terms of the experimental limitations and results by careful examination of the history of CW NIR retinal damage studies over a 40 year span.

Specific Aim 2: Quantify the strength and characteristics of a thermal lens in an eye by observing the phenomenon in a physical environment similar to the eye.

Specific Aim 3: Create a first-order mathematical model to simulate the process of forming a thermal lens.

Specific Aim 4: Establish retinal damage thresholds for CW NIR laser radiation wavelengths between 1100 and $1350-\mathrm{nm}$ in a rhesus eye.

Specific Aim 5: Predict the time-dependent temperature rise at the retina using the mathematical model to predict retinal damage threshold conditions for varying input beam diameters delivered to the cornea.

Specific Aim 6: Qualitatively describe the aberrations which arise from thermal lensing in a double-pass adaptive optics system and an artificial eye.

\subsubsection{Specific Aim 1}

Specify the scope of the problem with NIR retinal lesion studies in terms of experimental limitations and results by careful examination of the literature investigating CW NIR retinal damage studies conducted over a 40 year span (Chapter 2 - published in the Journal of Biomedical Optics, 2008). This includes the need to understand the concepts which the United States Army Medical Research Department, USAMRD, used to create their "action spectrum" to fit and predict the retinal damage threshold as a function of laser parameter variation.

The history of the NIR damage threshold studies is reviewed in detail, to include the explanation of how Lund et al., from the USAMRD, fit their "action spectra" to 
limited damage threshold data from the ultra-violet (UV) and into the NIR part of the spectrum. The key components which are used to create the action spectra lend insight into the formation of a lesion on the retina from NIR laser radiation and provide information about the current conventionally accepted ocular parameters for describing the laser-tissue interaction without thermal lensing.

\subsubsection{Specific Aim 2}

Quantify the strength and characteristics of a thermal lens in an eye by observing the phenomenon in a physical environment similar to the eye (Chapter 3). Using techniques similar to a confocal-imaging system, a series of wavelengths and power settings were sought to determine the natural response of the thermal lens in relation to time, power and wavelength.

A pump-probe laser system set-up, consisting of a visible-probe laser, was used to observe the beam-profile over time with a high-speed camera. Images were recorded at several axial positions inside an artificial eye, providing detailed information of the effect of thermal lensing upon the radial profile of the probe beam. Thermal lensing induced by 1313-nm CW laser radiation in a vitreous sample was compared to water to test the hypothesis that the thermal properties of vitreous are similar to those of water, confirming water to be a suitable test media for further investigation. Using an artificial eye, designed to have the same focal-length as that of the animal model used in retinal damage threshold studies conducted by the Air Force Research Lab, AFRL, the experiment was run for pump-laser wavelengths at 1110, 1130, 1150 and $1318 \mathrm{~nm}$ (Cain, Noojin, et al. 1997). This experiment provided valuable information for the steady-state and decay response of the thermal lens in relation to time, power and wavelength. 


\subsubsection{Specific Aim 3}

Create a first-order mathematical model to simulate the processes of forming a thermal lens (Chapter 4 - published in the Journal of the Optical Society of America-A, 2009). The model was used to explain trends in thermal lensing in terms of power, spotsize at the cornea and wavelength.

The mathematical model simulated the attenuation of a focused laser beam in the eye owing to heating and the resulting function of a transient thermal lens. This model was based upon existing models formulated for solid-state optics, but was the first of its kind to examine the effects of thermal lensing in the eye. A conventional z-scan spectroscopic experimental set-up was built and used to create experimental data in which to validate the thermal lensing model. Once a suitable fit of modeling results to experimental data were determined, the model provided predictions of the effect of thermal lensing on a beam profile throughout the eye.

\subsubsection{Specific Aim 4}

Establish retinal damage thresholds in a rhesus eye for continuous-wave, nearinfrared laser radiation wavelengths longer than 1100-nm (Chapter 5 - published in Lasers in Surgery and Medicine, 2009). Based upon available laser sources, a complete retinal damage threshold study was conducted at 1110, 1130 and 1319-nm laser-radiation wavelengths using 100-ms exposures. The action spectra concepts presented in Chapter 2 were used to provide a fitting algorithm for the retinal damage threshold results in Chapter 5.

\subsubsection{Specific Aim 5}

Model the temperature rise at the retina to predict retinal threshold conditions (Chapter 6). The first-order model, presented in Chapter 4, was further developed to 
simulate laser-radiation dose conditions of those determined to cause retinal damage from the damage threshold study presented in Chapter 5. The model's predicted temperature rise for known damage threshold parameter inputs were used as a point of reference to establish damage at the retina. A series of simulations with various wavelengths, beamdiameters and powers delivered to the corneal plane and exposure durations were compiled to determine when the damage threshold condition was achieved. Results from these simulations were then used to create a series of trends as to when the thermal lens may provide a protective factor for the retina. The first-order thermal lensing model, FOTLM, assumes thermal conduction is purely in the radial direction along the laserbeam's profile and neglects any heat exchange along the optical axis. Selected input parameter sets from the FOTLM were compared against a higher fidelity model, which included axial conduction, created by the AFRL, to validate that axial conduction can be neglected for certain input parameter conditions. Data from Chapter 6 are intended to be used as a guideline for experimental design to test the hypothesis of when thermal lensing protects the retina for future empirical and theoretical retinal damage threshold investigations at the AFRL.

\subsubsection{Specific Aim 6}

Observe thermal lensing in a double-pass optical system using an artificial eye (Chapter 7). An adaptive optics system is used to observe the thermal lensing effect in an artificial eye using an artificial retina. Results from this experiment indicate that the thermal lensing effect can be measured in vivo without damaging the retina. 


\section{CHAPTER 2}

\section{BACKGROUND}

\subsection{REVIEW OF CORNEAL AND RETINAL THRESHOLD STUDIES}

Retinal damage threshold studies have been conducted for wavelengths ranging from the ultraviolet (UV) to the infrared (IR) for continuous wave (CW) down to the ultra-short femtosecond pulses (Lund, Stuck et al. 1981; Yumita, Shirato et al. 1986; Brancato, Praresi et al. 1989; Cain, Toth et al. 1998; Lund, Edsall et al. 1998; ICNIRP 2000; Zuclich, Lund et al. 2004; Zuclich, Lund et al. 2007). Numerous studies have augmented retinal damage to observe discrete mechanisms contributing to the damage process, such as beam shape, retinal spot size and exposure duration (Zuclich, Lund et al. 1999; Cain, Noojin et al. 2003). The review presented in this chapter was published in Vincelette, Rockwell, et al., Journal of Biomedical Optics, 2008.

\subsubsection{Standard Procedures in Ocular Damage Threshold Studies}

Typically the primate, usually the Macaca mulatta commonly known as the rhesus, is preferred for careful in vivo retinal damage studies due to its eyes' close similarities to human eyes while a lesser species, the rabbit, is suitable for corneal damage studies and preliminary retinal damage studies (Polhamus and Welch 1975; Mikesell. 1978; Lund, Stuck et al. 1981; Birngruber, Gabel et al. 1983; Blankenstein, Zuclich et al. 1986; Cain, Toth et al. 1998; Lund, Edsall et al. 1998; Zuclich, Edsall et al. 2000; Zuclich, Lund et al. 2001; Zuclich, Lund et al. 2004; Peri, Turetz et al. 2006). Experiments using primates to evaluate retinal damage thresholds use a sedated subject; 
after the eye is cyclopleged and dilated, it is exposed to an incident laser beam of a selected wavelength for a pre-determined length of time (Lund, Stuck et al. 1981; Lund, Edsall et al. 1998; Zuclich, Edsall et al. 2000; Zuclich, Lund et al. 2004). For corneal damage studies, the beam is focused to approximately a $1-\mathrm{mm} 1 / \mathrm{e}$ beam diameter at the cornea. Frequently in retinal studies at any wavelength, the laser is passed through an aperture set to a 5-mm diameter for NIR and $3 \mathrm{~mm}$ for visible wavelengths, just before the cornea. The input beam diameters are kept small enough to avoid clipping of the pupil while still allowing the beam to focus in the model animal's natural position in a non-accommodated, emmetropic eye. As a result, most retinal damage studies do not correct for chromatic aberrations; longer wavelengths are focused at a more posterior retinal location than shorter wavelengths. For retinal damage studies, the laser radiation dose is recorded as the amount of energy entering the emmetropic eye, assuming no clipping of the beam on the pupil, per unit area and represented as the total intraocular energy (TIE). The TIE is used for convention since the actual amount of energy, or radiant exposure, reaching the retina is unknown. In comparison, corneal damage data are recorded in energy delivered at the cornea per unit area, but not referred to as TIE since the amount of energy reaching the target tissue is directly determinable. Retinal damage is assessed by using a fundus camera to observe the presence or absence of a lesion in a carefully marked and recorded grid on specific areas of the retina. If the lesion is visible, the size of the lesion can be estimated ophthalmoscopically or measured through histopathology. Three observers examine the area for lesions. A lesion is recorded as a positive read if at least two observers agree there is a lesion present. Of course the ability to see lesions at the retina is then dependent upon the fundus camera and the observer's eye-sight, leading to qualitative detection of the minimum visible 
lesion (MVL) which is discussed in section 2.2.3. Lesion data are related to exposure duration, wavelength, TIE (for the retina), corneal beam profile, elapsed time postexposure and other parameters using Probit analysis to assess the estimated threshold where damage occurs $50 \%$ of the time, known as an $\mathrm{ED}_{50}$, for a particular setting (Finney 1971; Cain and Noojin 1996). Once the $\mathrm{ED}_{50}$ is determined, the maximum permissible exposure, or MPE, is set by organizations such as the American National Standards Institute (ANSI) and the International Commission of Non-Ionizing Radiation Protection (ICNIRP), with a safety margin by a factor of three to 1000 based upon trends in experimental measurements.

\subsubsection{General Classes of Retinal Injury}

The damage observed at the retina can be divided into three classes; injury resulting from photochemical, thermal, or thermomechanical processes (Glickman 2002; Henderson and Schulmeister 2004). Photochemical processes are strongly wavelength dependent and are cumulative in nature. Damage from a photochemical mechanism involves a chemical change within the tissue resulting from shorter wavelengths, typically in the blue and UV.

Thermal processes involve injury arising from local increases in temperature in tissue as the direct result of light absorption (Henderson and Schulmeister 2004). Generally, wavelengths below $1200 \mathrm{~nm}$ are absorbed by melanin granules in the retinal pigment epithelium (RPE), just posterior to the neural retina. Damage from thermal processes is related to the radiant energy, and subsequently the spot size, linear absorption and the diffusion of heat from the RPE to the surrounding retinal tissues. This is believed to be the primary process involved in photocoagulation of the retina. 
Thermomechanical processes arise from short pulsed exposure durations, typically in the nanosecond regime, which can yield rapid heating of tissue causing localized thermal expansion and a photoacoustic wave generation in the media (Henderson and Schulmeister 2004). This mechanism can be further subdivided into photoablation and photodisruption. Photoablation is the result of ablating the surface of a tissue with a rapid heating process in such short time that tissue beyond the ablation boundary is left relatively undisturbed from the event. Photoablation is not a process in retinal damage, but can be seen in corneal damage effects. Photodisruption results from cases involving high peak irradiance and electric fields, similar to those seen in ultrashort pulses, causing the tissue to ionize and plasma formation inducing an acoustic wave.

\subsubsection{Summary of Corneal and Retinal Threshold Studies (1300-1356 nm), circa 1980-2004}

Damage threshold studies for the retina at wavelengths between 1300 and 1356 $\mathrm{nm}$, conducted by researchers at Brooks Air Force Base, Texas determined the $\mathrm{ED}_{50}$ of the retina for pulse durations of $650 \mu$ s or less in rhesus studies (Lund, Stuck et al. 1981; Zuclich, Lund et al. 2004; Zuclich, Lund et al. 2007). Retinal lesions were not observed for exposures longer than $650 \mu \mathrm{s}$ in the same species. In the rhesus, damage was observed in other portions of the eye for CW cases before any damage was created at the retina (Zuclich, Lund et al. 2001). Though retinal damage from a 1300-nm CW exposure with greater than $650 \mu$ s duration was never achieved in studies using rhesus primates, the same laser conditions caused retinal damage in rabbits. For a summary of retinal and corneal damage threshold studies see Tables 2.1 and 2.2 respectively. The ocular globe diameter of the rhesus monkey is approximately $11 \%$ longer than that of a rabbits 
(Hughes 1972; Li, Zwick et al. 2000; Fernandes, Bradley et al. 2003). Globe geometry details for the rhesus and rabbit eye are discussed in section 2.2.2.

Table 2.1: $\mathrm{ED}_{50}$ (reported for retina as a TIE) values for retina from $1315-1330 \mathrm{~nm}$. The TIE in $\mathrm{J}$ is the reported $E D_{50}$ measurement from literature. The TIE in $\mathrm{W} / \mathrm{cm}^{2}$ was calculated based on TIE(J)/(Pulsewidth $(\mathrm{sec})^{*}$ Approximate area at cornea $\left.\left(\mathrm{cm}^{2}\right)\right)$. The percent transmission was calculated from data reported by Maher 1978 and can be found in section 2.2.2.

\begin{tabular}{|c|c|c|c|c|c|c|c|}
\hline Species & $\begin{array}{c}\text { Wavelength } \\
(\mathrm{nm})\end{array}$ & $\begin{array}{c}\text { Pulse } \\
\text { Duration }\end{array}$ & $\begin{array}{c}\text { Approx. } \\
\text { Beam } \\
\text { Diameter } \\
\text { Entering }^{\text {Cornea }} \\
(\mathrm{mm})\end{array}$ & $\begin{array}{c}\text { TIE: } \\
\mathrm{ED}_{50} \\
(\mathrm{~J})\end{array}$ & $\begin{array}{c}\text { TIE } \\
\left(\mathrm{W} / \mathrm{cm}^{2}\right)\end{array}$ & $\begin{array}{c}\text { Predicted } \\
\% \mathrm{~T} \text { to } \\
\text { Retina }\end{array}$ & $\begin{array}{c}\text { Sources } \\
(\mathrm{d})\end{array}$ \\
\hline \multirow{3}{*}{ Rhesus } & 1319 & $50 \mathrm{~ns}$ & 4.5 & 0.0193 & $2.43 \mathrm{E}+06$ & 3.02 & 1,2 \\
\cline { 2 - 9 } & 1315 & $350 \mu \mathrm{s}$ & 5 & 0.334 & $4.86 \mathrm{E}+03$ & 3.78 & 1,3 \\
\hline \multirow{3}{*}{ Rabbit } & 1330 & $650 \mu \mathrm{s}$ & 5.5 & 0.356 & $2.31 \mathrm{E}+03$ & 1.39 & $1,3,4$ \\
\cline { 2 - 9 } & 1318 & $0.28 \mathrm{~s}$ & 5 & $\sim 34$ & $6.18 \mathrm{E}+02$ & 6.75 & 1,3 \\
\cline { 2 - 9 } & 1318 & $0.69 \mathrm{~s}$ & 5 & $\sim 34$ & $2.51 \mathrm{E}+02$ & 6.75 & 2,3 \\
\hline
\end{tabular}

(a) Note this measurement is taken in reference to the aperture placed some distance in front of a subject's cornea. The actual beam size entering the eye is unknown.

(b) Total Intraocular Energy (TIE) - Actual energy at cornea entering eye (assuming no clipping)

(c) This is the energy assumed to enter the eye at the cornea calculated by the formula: (TIE (J)/Pulse Duration (s) $) /\left(\pi *\right.$ Beam radius entering cornea $\left.{ }^{2}\right)$

(d) Sources:

${ }^{1}$ (Zuclich, Lund et al. 2004)

2 (Zuclich, Lund et al. 2007)

${ }^{3}$ (Zuclich, Lund et al. 2001)

${ }^{4}$ (Lund, Stuck et al. 1981) 
Table 2.2: $\mathrm{ED}_{50}$ values for cornea from $1315-1356 \mathrm{~nm}$.

\begin{tabular}{|c|c|c|c|c|c|c|c|}
\hline \multirow{5}{*}{ Species } & $\begin{array}{c}\text { Wavelength } \\
(\mathrm{nm})\end{array}$ & $\begin{array}{c}\text { Pulse } \\
\text { Duration }\end{array}$ & $\begin{array}{c}\mathrm{ED}_{50} \\
\left(\mathrm{~J} / \mathrm{cm}^{2}\right)\end{array}$ & $\begin{array}{c}\text { Corneal } \\
\text { Spot Size, } \\
\text { Diameter } \\
(\mathrm{mm})\end{array}$ & $\begin{array}{c}\mathrm{ED}_{50} \\
\left(\mathrm{~W} / \mathrm{cm}^{2}\right)\end{array}$ & $\begin{array}{c}\text { Lens } \\
\text { Relative } \\
\text { to } \\
\text { Cornea }^{(\mathrm{b})}\end{array}$ & $\begin{array}{c}\text { Sources } \\
(\mathrm{c})\end{array}$ \\
\hline \multirow{4}{*}{ Rhesus } & 1318 & $0.28 \mathrm{~s}$ & 72 & 1 & $2.57 \mathrm{E}+02$ & 1.8 & $1,2,5$ \\
\cline { 2 - 9 } & $1318 / 1338^{(\mathrm{a})}$ & $250 \mu \mathrm{s}$ & 45 & 0.4 & $1.80 \mathrm{E}+05$ & & 4,6 \\
\cline { 2 - 9 } & $1318 / 1338^{(\mathrm{a})}$ & $5 \mathrm{~s}$ & 212 & 1.4 & $4.24 \mathrm{E}+01$ & & 2,6 \\
\cline { 2 - 9 } & 1356 & $0.33 \mathrm{~s}$ & 81 & 0.7 & $2.67 \mathrm{E}+01$ & 5 & 2,5 \\
\hline \multirow{4}{*}{ Rabbit } & 1315 & $350 \mu \mathrm{s}$ & 42 & 1 & $1.20 \mathrm{E}+05$ & & $1-3$ \\
\cline { 2 - 9 } & 1318 & $0.69 \mathrm{~s}$ & 175 & 1 & $2.54 \mathrm{E}+02$ & 0.75 & 2,5 \\
\cline { 2 - 9 } & 1318 & $10 \mathrm{~s}$ & 1890 & 0.8 & $1.89 \mathrm{E}+02$ & & $1-3$ \\
\cline { 2 - 9 } & 1319 & $50 \mathrm{~ns}$ & 540 & 0.15 & $1.08 \mathrm{E}+10$ & & 1,2 \\
\hline
\end{tabular}

(a) $40 \% 1318,60 \%$ 1338. MPE based on $1330 \mathrm{~nm}$

(b) This value is a scalar; multiply by corneal $\mathrm{ED}_{50}$ for $\mathrm{J} / \mathrm{cm}^{2}$

(c) Sources:

${ }^{1}$ (Zuclich, Lund et al. 2004)

2 (Zuclich, Lund et al. 2007)

3 (Zuclich, Lund et al. 2001)

${ }^{4}$ (Lund, Stuck et al. 1981)

5 (Zuclich, Gagliano et al. 1995)

${ }^{6}$ (Stuck, Lund et al. 1980)

\subsubsection{Summary of Histological Findings}

Zuclich et al. report corneal damage at $1318 \mathrm{~nm}$ involved the full corneal thickness unlike earlier studies mentioned by Zuclich et al. in the far IR, where corneal damage only involved the epithelium layer (Zuclich, Blankenstein et al. 1984; Zuclich, Gagliano et al. 1995). In studies for 1315-1356 nm involving CW (0.1 - $1 \mathrm{~s})$ exposures, damage was observed at multiple sites throughout the eye including the cornea, lens, iris and retina in the rabbit (Zuclich, Gagliano et al. 1995). Similar observations were made in the rhesus save for the fact damage was never observed at the retina. Zuclich et al. 
also found the formation of cataracts in both rhesus and rabbit subjects when the incident power at the cornea was $260 \mathrm{~J} / \mathrm{cm}^{2}$ for $\mathrm{CW}(\sim 0.25 \mathrm{~s})$ exposures. Formation of the cataract was frequently observed to occur as two lesions in the eye, separated by a region of normal tissue. The first being an axially shallow lesion located at the anterior surface at the capsule, wherein the tissue immediately posterior to this lesion appeared normal. The second lesion, referred to as the cortical cataract, exhibited significant axial depth and involved up to one-third of the total lens thickness. The deeper cortical cataracts were noted to have an axially tapered lesion diameter. Zuclich et al. reported very few instances of cataracts for studies involving $350 \mu$ s pulses (Zuclich, Lund et al. 2001). In these $350 \mu$ s studies, damage through the anterior tissues of the eye was only observed when the beam was brought to a small spot of $1 \mathrm{~mm}$ or less at the surface of the cornea.

In NIR retinal $\mathrm{ED}_{50}$ studies, Zuclich et al. described the 1318-nm lesion process as involving the full retinal thickness which caused a late inflammatory response. A typical NIR retinal lesion did not appear within the first hour post-exposure (Zuclich, Gagliano et al. 1995; Zuclich, Schuschereba et al. 1997; Zuclich, Zwick et al. 1998; Zuclich, Lund et al. 2001). By 24-hours post-exposure, the NIR lesions were evident, characterized by a larger more reflective spot compared to the 532-nm argon marker lesions. NIR, 1300-nm retinal lesions for $1 \mathrm{~s}-10 \mathrm{~s}$ exposures are shown in comparison to argon threshold lesions in Fig. 2.1. 


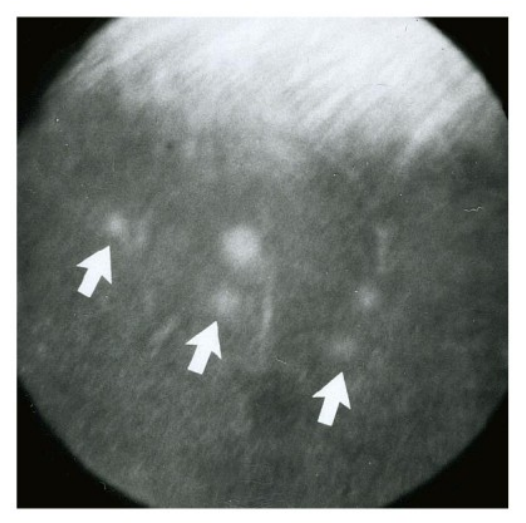

Figure 2.1: Fundus image of a rabbit subject (Zuclich, Schuschereba et al. 1997). The arrows indicate the 514-nm marker lesions. Lesions adjacent to the marker lesions are from CW (exposure lengths of $1 \mathrm{~s}-10 \mathrm{~s}$ ) 1318-nm NIR exposures as they appeared 24hours post-exposure. Zuclich et al. estimated that the argon lesions measured $200 \mu \mathrm{m}$ in diameter. This image first appeared in Zuclich, Schuschereba et al. "A comparison of laser induced retinal damage from infrared wavelengths to that from visible wavelengths." Lasers and Light in Ophthalmology. 8(1). p. 19 (1997).

The NIR lesions appeared to stabilize after 48 hours (Zuclich, Schuschereba et al. 1996; Zuclich, Schuschereba et al. 1997; Zuclich, Zwick et al. 1998). By comparison, the argon marker lesions were always immediately visible after the exposure and did not change significantly in appearance over time. The argon wavelength has much higher absorption within the RPE compared to the 1318-nm wavelength lesions. Pathology and imaging of minimum visible lesions, MVL, from this NIR region revealed minor damage reaching into the choroid with damage centered around the outer neural layer (ONL) (Zuclich, Schuschereba et al. 1997; Zuclich, Zwick et al. 1998). Swelling in the RPE was found to be much less in the 1318-nm NIR MVL studies compared to visible wavelength lesions. Zuclich et al. reported sub-threshold 1318-nm NIR MVL retinal exposures in rabbit subjects which were not visible in standard fundus camera observation, but were evident upon imaging with SLO at $780 \mathrm{~nm}$. Histology of these 
sub-threshold lesions, 48-hours post exposure, revealed some swelling in the inner retina, slight vacuolization in the RPE and dark nuclei in the ONL.

In observations made as long as two months post exposure, the NIR MVL shapes were irregular and large (Zuclich, Zwick et al. 1998). At three months post-exposure, the larger area of inflammation was no longer observed and the smaller circular NIR lesion which was first observed at 24 hours, appeared again.

\subsection{RETINAL DAMAGE THRESHOLd ACTION SPECTRUM}

Zuclich, Lund et al. 2007, Lund, Edsall et al. 2000, and Lund and Edsall 1999, have developed a process for expressing a theoretical fit to the TIE $\mathrm{ED}_{50}$ data as a function of wavelength based upon thermal and thermochemical ocular-tissue properties (Lund and Edsall 1999; Lund, Edsall et al. 2000; Zuclich, Lund et al. 2007). Though not by conventional definition, they refer to this theoretical fit as an action spectra. Their action spectra for the retinal hazard wavelengths $(400-1400 \mathrm{~nm})$ for 50 -ns and $0.1-\mathrm{s}$ exposures are compared to experimental $\mathrm{ED}_{50}$ data in Fig. 2.2. 


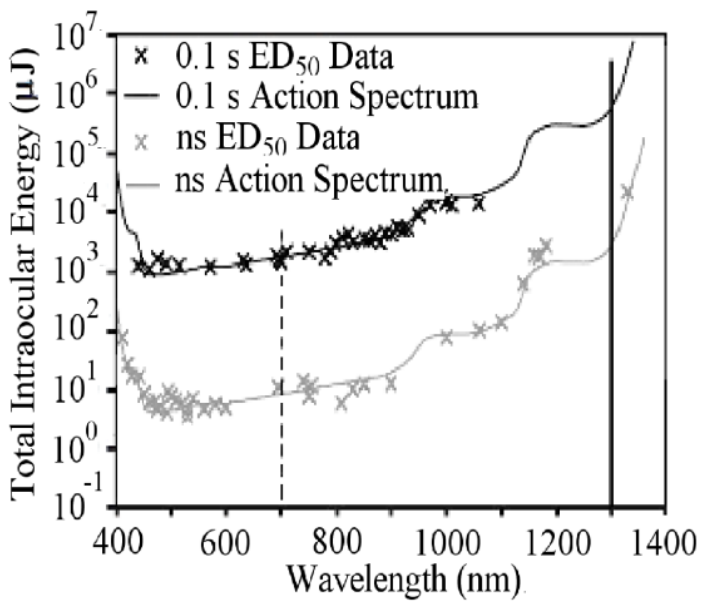

Figure 2.2: The total action spectrum for the rhesus eye for ns and 0.1-s exposures compared to corresponding experimental retinal $\mathrm{ED}_{50}$ data thresholds from 400-1350-nm wavelengths. Re-printed with permission from Zuclich, Lund et al. "Wavelength dependence of ocular damage thresholds in the near-IR to far-IR transition region: Proposed revisions to MPEs.” Health Physics. 92(1), p. 17. (2007).

The inherent complexities involved in the creation of the action spectra are best described by Lund and Edsall 1999, as a "simplistic model of the laser/tissue interactions and that the fit may be in part fortuitous." It should be understood that the predicted action spectra is a heuristic approximation to $\mathrm{ED}_{50}$ data. Nonetheless, the algorithm for creating the action spectra provides a classic foundation for analyzing the laser tissue interaction in ocular media (Lund and Edsall 1999; Lund, Edsall et al. 2005).

The calculation for the action spectra is first referenced to a fixed data point using the TIE measured from the experimentally determined ED $_{50}$ MVL for 700-nm light at 0.1s and 50-ns exposure durations, marked by the dashed vertical line in Fig. 2.2. Below $1200 \mathrm{~nm}$, the 50-ns exposures fall within the thermal impulse response of the RPE to laser irradiation and the 0.1-s exposures approach the steady state thermal response (Priebe and Welch 1978). The $\mathrm{ED}_{50}$ TIE estimates for all wavelengths, other than 700$\mathrm{nm}$, are then calculated based on three fundamental criteria: 1) absorbance of the RPE 
and water, 2) percent transmission of incident light to the retina, and 3) the predicted spot size at the retina primarily based on chromatic dispersion models. The greatest uncertainty with NIR predictions is the spot size of the laser beam at the retina and the role of RPE absorption in retinal heating. Fig. 2.2 includes the experimental TIE $\mathrm{ED}_{50}$ data and the computed action spectra for $0.1-\mathrm{s}$ and ns pulse durations.

The action spectra for 0.1 -s and ns exposure durations have been shown to fit the respective TIE data well for wavelengths below $1200 \mathrm{~nm}$ (Zuclich, Lund et al. 2007). A gap in data exists between 1064 and $1350 \mathrm{~nm}$, leaving this area of the NIR largely unexplored. The solid vertical line in Fig. 2.2 marks the only area in this gap, the 1300$\mathrm{nm}$ region, which has been investigated. A summary of results from previous studies in this 1300-nm region is described in section 2.1.3. Perspective of the thermal lensing problem with safety thresholds in the NIR region from $1150-1350 \mathrm{~nm}$ can be gained by examining the three fundamental criteria used in developing the action spectra.

\subsubsection{Criteria 1: Absorbance of the RPE and Water Content of the Retina in the NIR Wavelength Region}

Characterizing the absorbance of the RPE has been an arduous process for the laser-tissue community. Though some fluorophores and their isomers have been studied, to date, the absorptive properties of the RPE in the NIR are largely unknown (Eldred and Katz 1988; Vitkin, Woosley et al. 1994; Nofsinger, Forest et al. 1999; Nofsinger and Simon 2001; Fishkin, Sparrow et al. 2005). Numerous physiological changes occur in the aging RPE including a reduction in cell density and changes in pigmentation (Strauss 2005). One cause in the pigmentation change is from lipofuscin, a fatty by-product of metabolic processes containing pigments, which accumulates in RPE cells over time (Fishkin, Sparrow et al. 2005). Lipofuscin build-up is just one example which can cause variability in optical absorption between species and subjects. In lieu of these facts, 
characterization of reported chromophores such as eumalanin has revealed a significant decrease in absorption towards longer wavelengths (Vitkin, Woosley et al. 1994; Nofsinger, Forest et al. 1999; Nofsinger and Simon 2001). Given the variability in pigmentation content and density of the retina between subjects, a trend in absorption of the RPE, neural retina and choroid in the NIR is a suitable analysis. In the 1960's1980 's, numerous scientists sought to measure the absorption of the retina or RPE over a variety of wavelengths; finding a large decrease in absorbance at longer wavelengths (Geeraets, Williams et al. 1960; Geeraets, Williams et al. 1962; Coogan, Hughes et al. 1974; Gabel, Birngruber et al. 1978; Birngruber, Hillenkamp et al. 1985). To develop the action spectra in Fig. 2.2, Lund and Edsall 2000, determined the data from Gabel et al. and Birngruber et al. yielded the best fit to MVL data (Gabel, Birngruber et al. 1976; Gabel, Birngruber et al. 1978; Birngruber, Hillenkamp et al. 1985). A least squares fit to the Birngruber, Hillenkamp et al. 1985 data gives (see Eq. 2.1) (see Fig. 2.3)

$$
\% A=458 * \exp \left(-4.3 * 10^{-3} \lambda\right) \text {. }
$$

The $\% \mathrm{~A}$ is the percentage of absorbed energy by the RPE (based on energy reaching the RPE layer, not on TIE), and $\lambda$ is the wavelength with units of nm. 


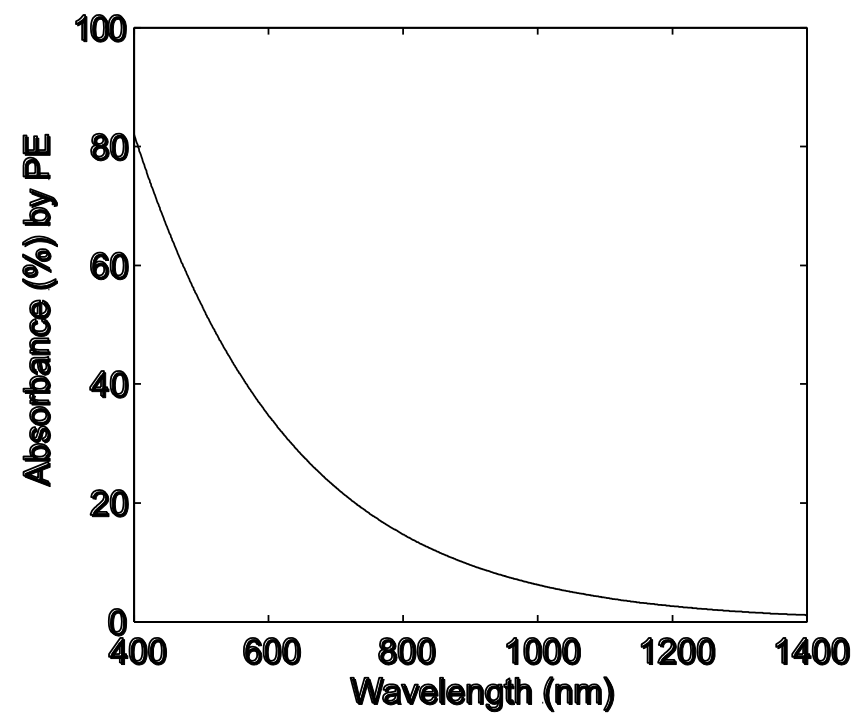

Figure 2.3: A fit to the absorption data of rhesus PE from Birngruber, Hillenkamp et al.1985 data described by Eq. 2.1.

A more detailed fit used in the action spectrum uses the general form for RPE absorbance as $1-\exp \left(-\mu_{\mathrm{a}}{ }^{*} \mathrm{~s}\right)$ with all wavelengths normalized to the RPE absorption at 380 $\mathrm{nm}$ (Lund, Edsall et al. 2008). Where $\mathrm{s}$ is the path length of the melanin granules, set to $5 \mu \mathrm{m}$, and $\mu_{\mathrm{a}}$ is the linear absorption coefficient calculated by $\mu_{\mathrm{o}}\left(\lambda_{\mathrm{o}} / \lambda\right)^{3.5}$. The $\lambda_{\mathrm{o}}$ is the normalizing wavelength of $380 \mathrm{~nm}$ with a corresponding $\mu_{\mathrm{o}}$ of $4100 \mathrm{~cm}^{-1}$.

In the visible region, threshold lesions remained centered on the RPE, though suprathreshold exposures damaged the full-retinal thickness. In contrast, NIR threshold lesions involving full-retinal thickness and late-onset inflammation were not observed at visible or UV wavelengths (Zuclich, Schuschereba et al. 1997)

In general, the sensory retina is mostly transparent to retinal hazard wavelengths (Henderson and Schulmeister 2004). Rich with melanin granules, the RPE is believed to absorb most of the energy in the visible range. In contrast, wavelengths in the NIR penetrate into the choroid which contains blood vessels and some melanin granules. Geeraets et al. reported bulk absorbance of human retina and choroid over a broad spectrum finding a mean value of approximately $35-40 \%$ absorbance in the $1200-1400$ 
$\mathrm{nm}$ region (Geeraets, Williams et al. 1960). Considering the results from Geeraets, William et al.1960 and the fit to the Birngruber, Hillenkamp et al. 1985 data in Fig. 2.3, a trend can be seen where PE absorption decreases in the NIR while bulk absorption of the whole retina and choroid is much larger than in the RPE. It is reasonable to assume if initial denaturation occurs throughout the retina, owing to local heating of absorbed NIR light, then the average RPE absorption coefficient is not significantly larger than the value of water in the NIR region. Denaturation of the retina from radiation wavelengths in the NIR would not result from heat conducted from the RPE which is the mechanism for retinal coagulation using laser light in the visible spectrum. Instead, denaturation of the retina is due to the local water absorption of the NIR-laser beam, with RPE absorption being only slightly higher.

\subsubsection{Criteria 2: Transmission of Laser Radiation to the Retina}

One of the differences between rabbit and rhesus in the 1300-nm retinal thresholds listed in Table 2.1 lies in the path length for each species. The axial lengths of an adult rabbit and rhesus eye are 1.79 and $1.99 \mathrm{~cm}$ respectively (Hughes 1972; Fernandes, Bradley et al. 2003). Transmission losses include specular reflections and attenuation throughout the ocular media, refer to Fig. 2.4. Expectedly the amount of 1300-nm light which reaches the retina is greater for the rabbit compared to the rhesus (see Table 2.4). 


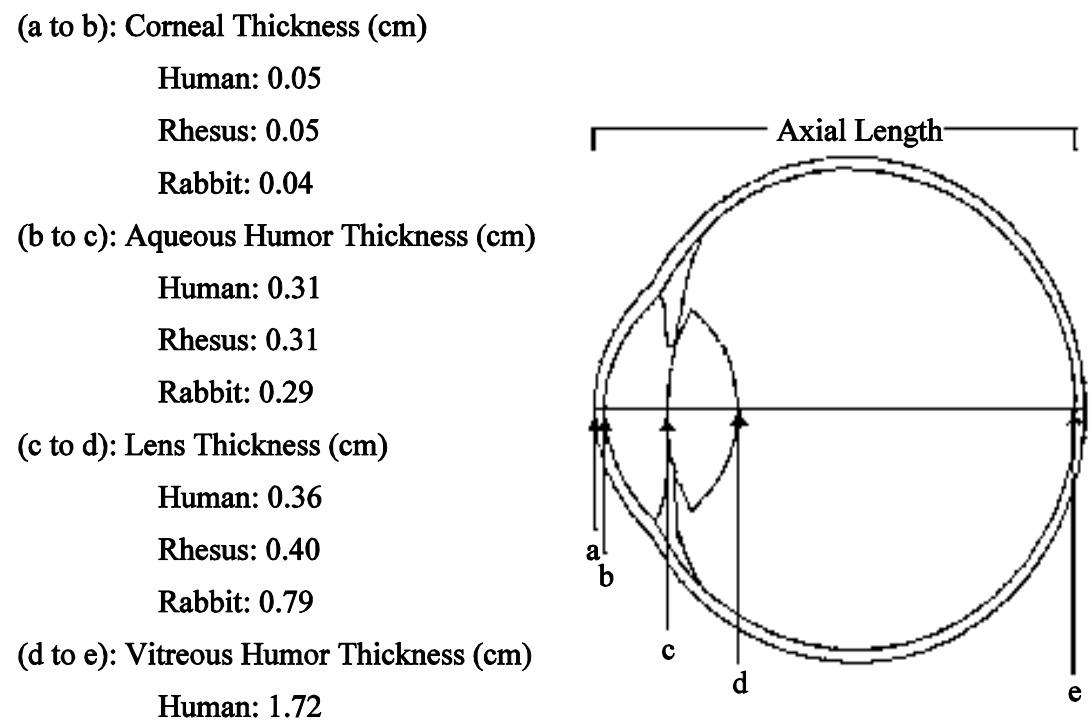

Rhesus: 1.23

Rabbit: 0.67

Figure 2.4: Physiological data for eyes of three species represented as comparable schematic eyes (Hughes 1972; Westheimer 1980; Li, Zwick et al. 2000; Fernandes, Bradley et al. 2003). Axial length is the sum of the distances from the front of the cornea at position a to the front of the retina at position e. Note the human lens thickness data includes the cortex, core and post-cortex for a total lens.

Absorption as a function of wavelength is slightly different for each portion of the eye as indicated by the measurements reported by Maher 1978, (see Fig. 2.5) and follow closely to the absorption of water as reported by Hale and Querry 1973, in the NIR region. Absorption coefficients in the 1300-nm region for each ocular medium are reported in Table 2.3. 


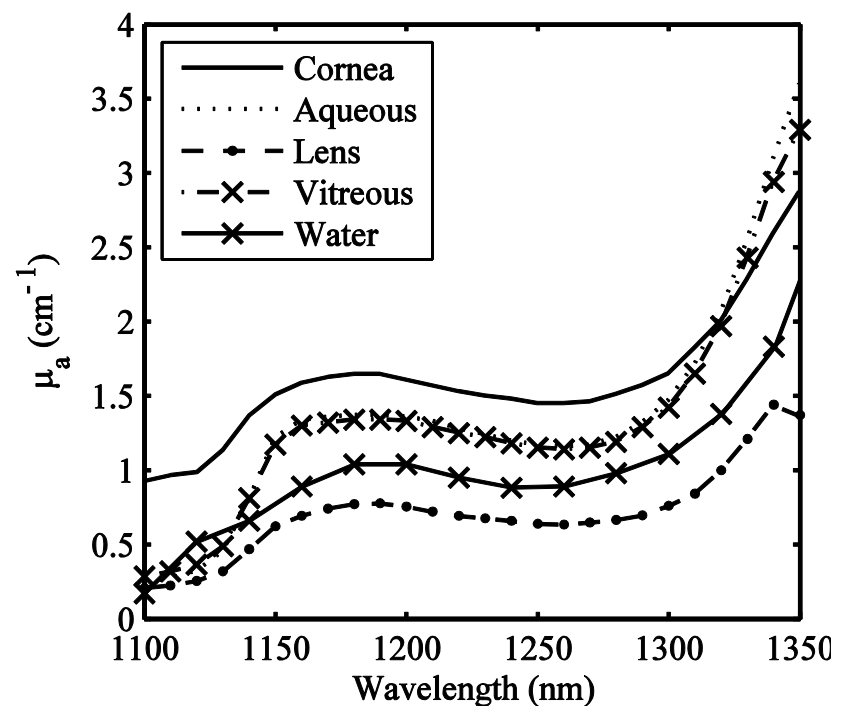

Figure 2.5: Linear absorption coefficient, $\mu_{\mathrm{a}}$, as a function of wavelength for ocular media from rhesus eyes and water (Hale and Querry 1973; Maher 1978).

Table 2.3: Linear absorption coefficient, $\mu_{\mathrm{a}}\left(\mathrm{cm}^{-1}\right)$, in the $1300-1330 \mathrm{~nm}$ region for ocular tissues interpolated from the data of Maher 1978.

\begin{tabular}{|c|c|c|c|c|}
\cline { 2 - 5 } \multicolumn{1}{c|}{} & \multicolumn{4}{c|}{$\lambda(\mathrm{nm})$} \\
\cline { 2 - 5 } \multicolumn{1}{c|}{} & 1300 & 1315 & 1318 & 1330 \\
\hline Comea & 1.65 & 1.92 & 1.98 & 2.28 \\
Aqueous & 1.47 & 1.90 & 2.01 & 2.54 \\
Lens & 0.761 & 0.92 & 0.97 & 1.21 \\
Vitreous & 1.42 & 1.80 & 1.90 & 2.43 \\
\hline
\end{tabular}

For creating the action spectrum in Fig. 2.2, Lund and Edsall 1999, reported using the values from Maher 1978, with approximately 5-10\% transmission to the RPE layer at or near $1300 \mathrm{~nm}$ wavelengths. If the physiological data from Fig. 2.4 are considered along with the linear absorption coefficients presented in Fig. 2.5, then Beer's law of attenuation can be applied to predict the percentage of light transmitted to the retina as seen in Fig. 2.6. Percent transmission values to the RPE for human, rhesus and rabbit in the $1300-\mathrm{nm}$ region are reported in Table 2.4. 


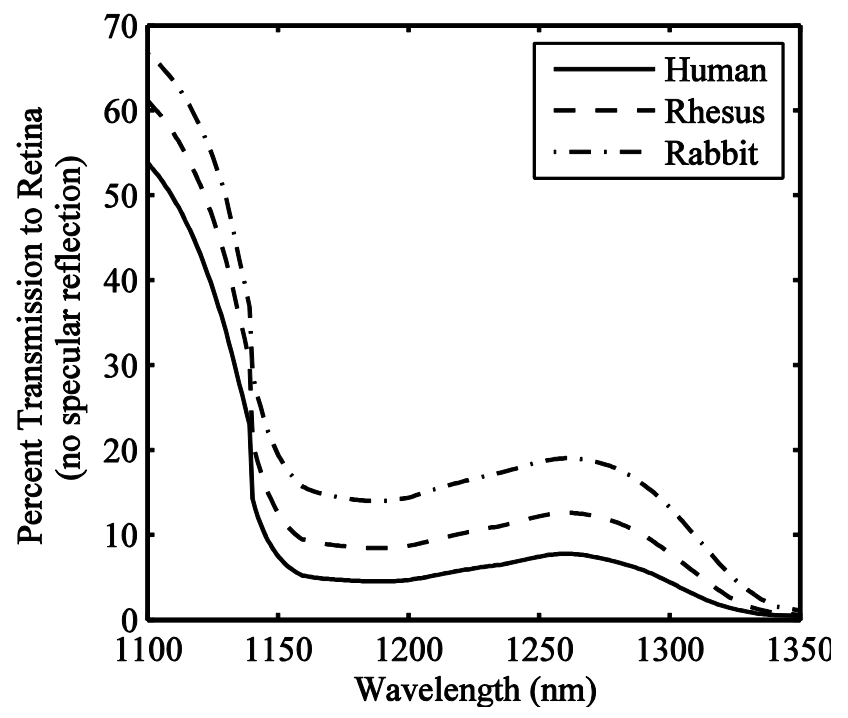

Figure 2.6: Percent of transmitted light reaching the retina in the schematic eye from Figure 2.4. Transmission values were calculated by Beer's Law using ocular absorption values from Fig. 2.5 for respective ocular components. Calculations used here did not account for specular reflections at any boundary.

Table 2.4: Percent of light transmission to RPE for rabbit, rhesus and human eyes based on results from Fig. 2.6.

\begin{tabular}{|c|c|c|c|}
\hline$\lambda(\mathrm{nm})$ & Rabbit & Rhesus & Human \\
\hline 1300 & 12.93 & 7.50 & 3.86 \\
1315 & 7.74 & 3.83 & 1.65 \\
1318 & 6.72 & 3.18 & 1.30 \\
1330 & 3.35 & 1.27 & 0.40 \\
\hline
\end{tabular}

The fact that damage was induced at the retina in rhesus monkeys for 1330-nm exposures of $650 \mu \mathrm{s}$ or less indicates there was sufficient transmission to the retina to cause damage in the rhesus, but does not explain why damage was not induced for CW exposures. It should be noted that the 1318-nm CW laser experiments were constrained to $20-\mathrm{W}$ maximum power, with only $10 \%$ of the maximum power reaching the corneal plane. Zuclich, Lund et al. 2001 and Zuclich, Gagliano et al. 1995, reported the occurrence of anterior damage in multiple locations for both rhesus and rabbit in cases 
where the incident beam at the cornea was less than $1 \mathrm{~mm}$. Rabbit retina $\mathrm{CW}$ exposures were conducted at $1318 \mathrm{~nm}$ and retinal lesions were observed.

To date, no other wavelength study has yielded such a discrepancy between species where $\mathrm{CW}$ retinal lesions could be seen in one but not the other. It is reasonable to assume differences between the rabbit and rhesus globe diameters' contributes to the presence or absence of a lesion. Still, these differences do not afford a complete explanation in the scope of all three criteria to compute an action spectrum. Speculation on the impact of these physiologic differences on threshold data is saved for discussion.

\subsubsection{Criteria 3: Prediction of Spot Size Using Chromatic Dispersion Models}

Lund, Edsall et al. 2005, described the ratio of the diameter of the chromatic spot, $\mathrm{d}_{\lambda}$, to that of the referenced minimum visible lesion, MVL, wavelength held as a constant, $\mathrm{d}_{0}$, as the only time-dependent quantity in the computation of the action spectrum shown in Fig. 2.2. This ratio is expressed in the action spectrum computation by

$$
\left(\frac{d_{\lambda}}{d_{0}}\right)^{x}
$$

The power $\mathrm{x}$ is a weighting factor ranging from a value of 2 , for ns pulses, down to a value of 1 , for $0.1 \mathrm{~s}$ or longer exposures. Controversy has surrounded the value of $\mathrm{x}$ due to the debate on the value of $\mathrm{d}_{0}$. Typically, $\mathrm{d}_{0}$ has been set to $25 \mu \mathrm{m}$, but some more recent studies suggest $\mathrm{d}_{0}$ is two-to-three times larger (Zuclich, Lund et al. 1999; Zuclich, Edsall et al. 2000; Zuclich, Lund et al. 2002; Lund, Edsall et al. 2007). The determination of the presence of a lesion is subject to error from the fundus camera, animal subject's eye and the observer (to include the observer's eye sight and experience level with reading lesions). It is worthwhile to detail what the term MVL truly implies. 
In studies designed to determine the threshold for laser-induced retinal injury as a function of beam diameter at the retina, Lund et al. noted the appearance of a lesion diameter did not decrease for irradiance diameters less than 80-100 $\mu \mathrm{m}$ (Lund, Edsall et al. 2007) Some proposed hypotheses include forward scattering properties of the eye, uncompensated ocular aberrations and the fact that a RPE cell is about $13 \mu \mathrm{m}$ in diameter. Essentially, the referenced MVL establishing the value for $\mathrm{d}_{0}$ represents the diameter of the lesion formed by the denatured retina after a threshold exposure to the reference wavelength of $700 \mathrm{~nm}$. The denatured area found by fundus camera observation is created by the thermal profile induced in the tissue from the laser-beam's waist at the retina. Thus, if a minimum beam waist is predicted to be smaller than the value of $\mathrm{d}_{0}$, theoretically, the MVL would still be no smaller than this referenced diameter. For this reason, creating the action spectra requires placing a lower bound of $\sim 40 \mu \mathrm{m}$ on retinal diameter.

To calculate $\mathrm{d}_{\lambda}$, Lund and Edsall et al. 2000, use chromatic dispersion for a simple reduced eye model using the refractive error measurements from Wald and Griffen, and Bedford and Wyzecki (Wald and Griffen 1947; Bedford and Wyszecki 1957; Lund and Edsall 1999). More recent chromatic dispersion data, or refractive error, in the NIR for the human eye has been published (Fernández, Unterhuber et al. 2005).

Chromatic dispersion of the human eye is typically represented by refractive error measurements, wherein, all wavelengths are described by their relationship to a referenced wavelength of $589 \mathrm{~nm}$ (The Eye: Visual Optics and Optical Space Sense. (1962)).

Refractive error, $\mathrm{R}$ [Diopters, $\mathrm{D}$, equivalent to $\mathrm{m}^{-1}$ ], is related to the reference focal length, $\mathrm{f}_{0}[\mathrm{~m}]$, and the focal length of other wavelengths, $\mathrm{f}[\mathrm{m}]$, by 


$$
R=\frac{1}{f_{0}}-\frac{1}{f}
$$

where $1 / f_{0}$ is the power of the eye, $D_{0}$ [Diopters], at the reference wavelength and $1 / f$ is the power of the eye, D [Diopters], at the wavelength being compared.

Thomas et al. use a reduced eye model with radius of curvature of $6.1 \mathrm{~mm}$ and $1 / \mathrm{e}^{2}$ input beam diameter of $4.2426 \mathrm{~mm}$ giving a focal length of $24.4 \mathrm{~mm}$ for the $589-\mathrm{nm}$ wavelength (Thomas, Vincelette et al. 2007). Using this reduced eye model, a solution for the index of refraction as a function of wavelength can be found in a three step process. First, Eq. 2.3 is used to generate values for $f(\lambda)$ using the reduced eye model for $\mathrm{f}_{0}$ and the refractive error, R, values given by Liou and Brennan 1997, and Fernandez, Unterhuber et al. 2005. The ABCD transform for a spherical-dielectric interface is then used with radius, $r=6.1 \mathrm{~mm}$, to converge upon a solution to $\mathrm{n}(\lambda)$ with the index of refraction of air set to 1.0. Finally, a Sellmeier equation with the general form

$$
n^{2}(\lambda)=1+\frac{B_{1} \lambda^{2}}{\lambda^{2}-C_{1}}+\frac{B_{2} \lambda^{2}}{\lambda^{2}-C_{2}}+\frac{B_{3} \lambda^{2}}{\lambda^{2}-C_{3}},
$$

is fit to the result for $n(\lambda)$. The Sellmeier equation is then used to generate the ocular dispersion data needed for the reduced eye model. Fig. 2.7a shows refractive error of the human eye and Fig. 2.7b shows the resulting solution to $n(\lambda)$ using the values of $R$ from Liou and Brennan 1997, and Fernandez, Unterhuber et al. 2005. The resulting Sellmeier coefficients for $n(\lambda)$ are given in Table 2.5. 
(a)

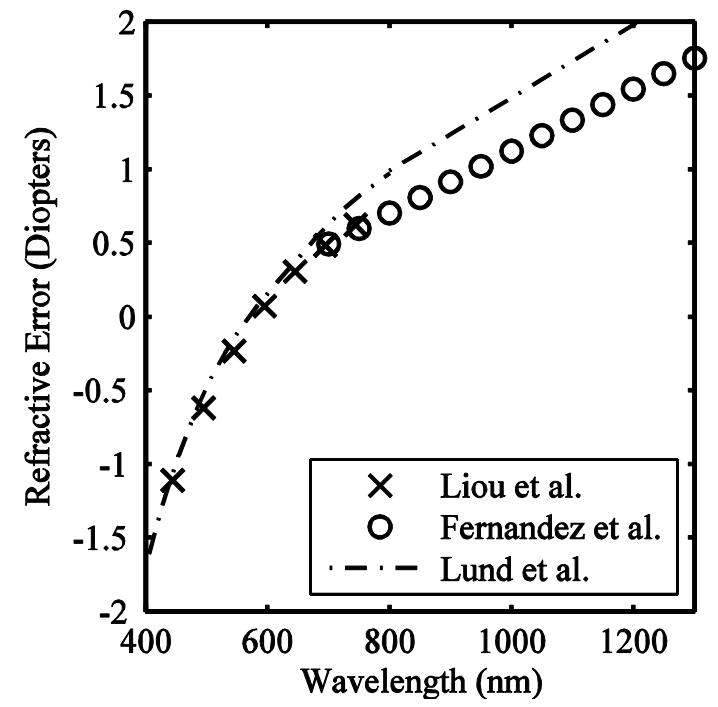

(b)

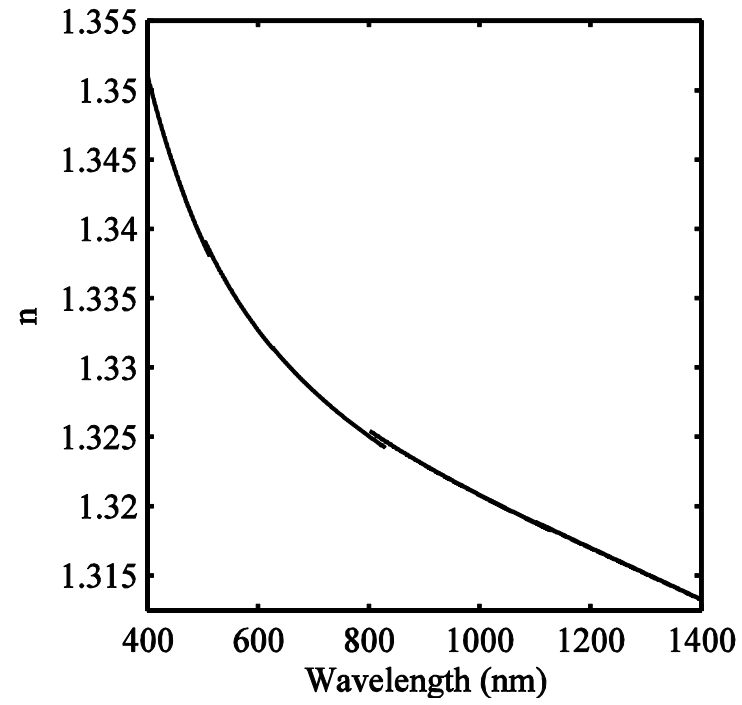

Figure 2.7: (a) Compilation of refractive error of the human eye for several summary reports including an estimate of refractive error for computing the results by Lund, Edsall et al. 2000 (Liou and Brennan 1997; Lund and Edsall 1999; Atchison and Smith 2005; Fernández, Unterhuber et al. 2005). The linear fit from the Fernandez, Unterhuber et al. 2005 data was shifted to match the refractive error of the Liou and Brennan 1997, modeled at $700 \mathrm{~nm}$. (b) Result for $\mathrm{n}(\lambda)$ based on the Liou and Brennan 1997, and Fernandez, Unterbuber, et al. 2005 data from (a). The Sellmeier fit to produce this result used the coefficients given in Table 2.5.

Table 2.5: Sellmeier coefficients found to produce a fit to the iterative solution for $n(\lambda)$, see Fig. 2.7b, based upon the refractive error values of the human eye from Liou and Brennan 1997, and Fernandez, Unterhuber et al. 2005, in Fig. 2.7a.

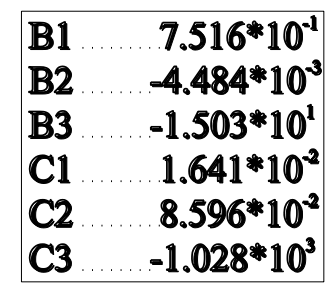

The fit reported by Fernandez, Unterhuber et al. 2005, suggests earlier refractive error in the NIR region has been over-estimated (see Fig 2.7a). Taking the dispersion data from Fig. $2.7 \mathrm{~b}$ in conjunction with the reduced eye model by Thomas, Vincelette $e t$ al., 2007, the spot size at a virtual retina can be predicted. The position of the virtual 
retina, or focal plane, is chosen to be the position for where refractive error measurements are referenced, or where $589-\mathrm{nm}$ comes to a minimum focus. The computational chromatic spot size as a function of wavelength is presented in Fig. 2.8.

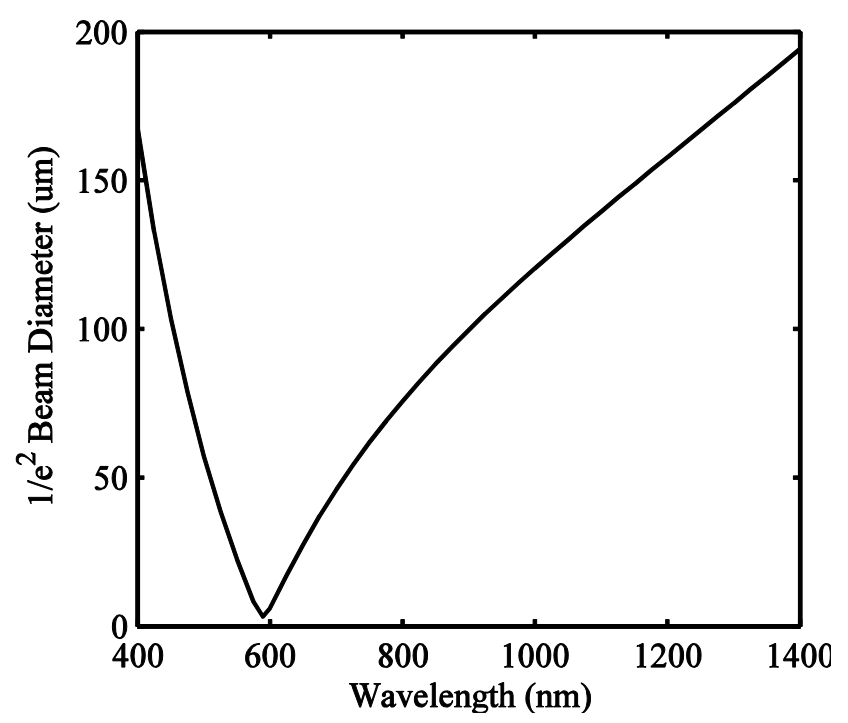

Figure 2.8: Predicted spot size at a virtual retinal plane using the information from the reduced eye model in conjunction with the dispersion data from Fig. 2.7b.

From Fig. 2.8, the expected $1 / \mathrm{e}^{2}$ spot diameter of a $1300-\mathrm{nm}$ beam is on the order of $180 \mu \mathrm{m}$. However, the chromatic spot size prediction is strongly dependent upon the input parameters including dispersion and the beam profile being launched into the eye model at the virtual cornea. The distance between the focal positions of the reference wavelength and the 1300-nm wavelengths can be calculated from the equation for refractive error yielding a distance of approximately $520 \mu \mathrm{m}$ for a relaxed, 58D eye. For a retina approximately $200 \mu \mathrm{m}$ thick, the focus of NIR wavelengths reach the choroid.

\subsection{DISCUSSION}

NIR retinal damage threshold lesions in the $1300-\mathrm{nm}$ region characteristically involve the full-retinal thickness and exhibit delayed onset inflammation (Zuclich, 
Schuschereba et al. 1997; Zuclich, Lund et al. 2007). Understanding the nature of 1300$\mathrm{nm}$ retinal damage thresholds is aided by considering the beam's focus resides in the choroid due to chromatic dispersion and the strong absorptive properties of water in the NIR. One unexplored mechanism of CW NIR retinal damage is the protection of the retina and choroid by a thermal lens gradient. The thermal lens causes the focus of the beam to shift posterioraly, increasing the beam size on the retinal plane, and subsequently, decreasing the fluence rate $\left[\mathrm{W} / \mathrm{cm}^{2}\right]$ in the neural retina, RPE and choroid. Lin et al. have demonstrated how thermal lensing is strongly related to the absorption coefficient suggesting thermal lensing plays a role in tissue optics (Lin, Motamedi et al. 1995; Lin 1997). Predicting thermal lensing is present during the $1300-\mathrm{nm}, \mathrm{CW}$ exposures is reasonable given the large absorption coefficient of ocular media in the 1300-1340 nm threshold studies (Maher 1978).

The role of thermal lensing as a NIR damage mechanism may be significant in wavelengths where less than $50 \%$ of the TIE reaches the retina, or absorption coefficients reach the order of $1 \mathrm{~cm}^{-1}$ (see Fig.'s 2.5 and 2.6). In this region damage to the retina becomes less dependent upon RPE absorption and more dependent on thermal diffusion of the bulk absorbance due to water content.

Typically, the phenomenon of thermal lensing is exploited in a z-scan technique to quantify weak or strong absorbance properties of a liquid or material (Franko and Tran 1996). Thermal lensing can be easily demonstrated by projecting the transmitted light from a collimated or focused beam through an absorbing medium onto a screen in the farfield. Images of how a focused spot in vitreous changes in time are illustrated in Fig. 2.9. 


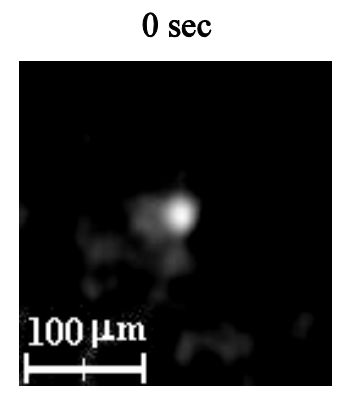

$0.047 \mathrm{sec}$

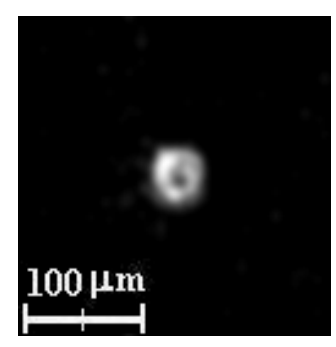

$0.020 \mathrm{sec}$

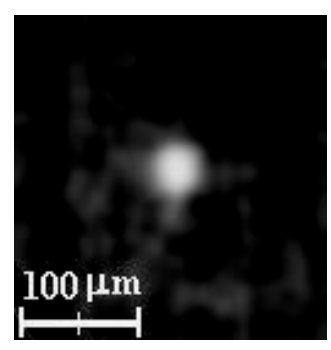

$0.127 \mathrm{sec}$

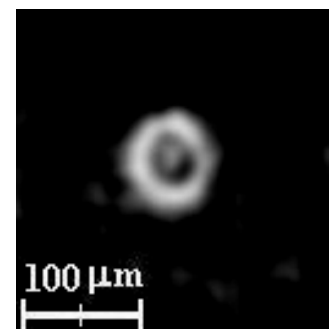

Figure 2.9: Changes in a minimum spot formed by a 25.4- $\mathrm{mm}$ focal length lens for a $633-\mathrm{nm}$ beam co-aligned with a $1313-\mathrm{nm}$ beam passing through a 1-cm thick sample of vitreous $\left(\mu_{\mathrm{a}}=1.77 \mathrm{~cm}^{-1}\right)$ at $0,20,47$ and $127 \mathrm{~ms}$. The spot begins to diverge, or bloom, becoming non-linear after a period of time in the exposure depending on absorptive properties of the sample and input power (Vincelette, Thomas et al. 2007).

Gordon, Leite et al. 1965, described the governing equation for changes in the local index of refraction, $\mathrm{n}$, in terms of radial distance vector, $\vec{r}$, from the central axis and temperature rise, $\Delta \mathrm{T}$, over time, $\mathrm{t}$, as described as (see Eq. 2.5)

$$
n(\vec{r}, T)=n_{0}+\left(\frac{d n}{d T}\right) \Delta T(\vec{r}, t)
$$

The term $\mathrm{n}_{\mathrm{o}}$ is the background index of refraction at $\mathrm{t}=0$. Gordon, Leite et al. 1965, demonstrated how even a weakly absorbing media with a $\mu_{\mathrm{a}}$ of $10^{-3}$ to $10^{-4} \mathrm{~cm}^{-1}$ and a small $0.075{ }^{\circ} \mathrm{C}$ increase in temperature resulted in the formation of a thermal lens with a $7.5 * 10^{-5}$ change in $\mathrm{n}$ from beam center to beam edge. The radial temperature gradient increases as the power and absorption coefficient increase, thus the strength of the 
thermal lensing effect is predicted to increase. The decrease in index of refraction as temperature increases in ocular components, combined with the Gaussian radial profile of the laser beam (and thus initially a Gaussian heating profile in the radial direction), creates a negative lens effect (Yariv 1989).

The time dependent ratio of $\mathrm{d}_{\lambda}$ to $\mathrm{d}_{0}$ as seen in Eq. 2.2 has been debated based upon the value of $\mathrm{d}_{0}$. Retinal lesions smaller than $40 \mu \mathrm{m}$ may be unseen by the fundus camera as found in earlier studies (Lappin and Coogan 1970; Welch and Polhamus 1984). The value of $d_{0}$ is subject to variability including limitations of the ophthalmoscope, proper training and experience of the observer, and contrast variations in pigmentation between subjects' retinas lending to lesion appearance.

The value of $d_{\lambda}$ needs to be examined as well. The value of $d_{\lambda}$ will be dependent upon the transient response of the thermal lensing gradient. $\mathrm{ED}_{50}$ data in the ultrashort regime represent an impulse response with negligible thermal lensing. Data for $\mathrm{CW}$ exposures, however, represent a steady state response with sufficient time for a thermal lens gradient to form.

Many laser-tissue mechanisms from an eye exposed to NIR laser radiation can be explained through the analysis of an action spectrum. These include the strong linear absorbance in ocular tissues leading to decreased transmission to the retina, weaker absorption of NIR in the RPE layer in the retina, and chromatic dispersion in the eye resulting in a larger spot on the retinal surface with damage through the full-retinal thickness. In addition to these mechanisms, thermal lensing may afford some protection for the rhesus eye from retinal damage at $\mathrm{CW}$ exposures helping to explain the variance in results between rabbit and rhesus retinal damage studies. The protective effect is not unconditional, though the condition for where CW NIR radiation causes retinal damage 
in a rhesus subject has only recently been determined and is reported in Chapter 5. Thermal lensing in the NIR has been an unexplored mechanism in ocular media. A goal of this research is to investigate what magnitude the thermal lensing effect has, if any, in the eye in protecting the retina and choroid from damage.

\subsection{ACKNOWLEDGEMENTS}

The author would like to thank Gary Noojin, with Northrop Grumman, for his assistance in calculating the Sellmeier Coefficients in this chapter. Thank you to my sponsors at the Air Force Research Laboratory and to Mr. David Jack Lund and Mr.

Bruce E. Stuck at the Army Research Laboratory for all their assistance in piecing together this fundamental overview of a complicated problem. 


\section{CHAPTER 3}

\section{CONFOCAL IMAGING OF THE THERMAL LENSING EFFECT IN AN ARTIFICIAL EYE}

\subsection{ABSTRACT}

A confocal imaging system mounted to a micrometer stage was used to image the thermal lens induced in an artificial eye. A dual-beam pump-probe geometry was used to quantify the 633-nm visible wavelength probe-beam's transient response when exposed to the near-infrared pump-beam source. The first portion of the study sought to compare the thermal lens of vitreous humor to water exposed to an available 1313-nm laserradiation for 1 -s exposures. The density of vitreous was measured to be $5 \%$ greater than that of water and determined not to denature from a temporarily available continuouswave, CW, 1318-nm wavelength laser radiation source at power levels up to the limits of the laser. Thermal lensing data for the 1313-nm wavelength determined water and vitreous produce similar thermal lens effects suggesting water is a suitable substitute for vitreous in thermal lensing investigations pertaining to the eye. Infrared laser radiation wavelengths tested were $1110,1130,1150$, and $1318 \mathrm{~nm}$ for 1-s exposures with power levels ranging from 130-890-mW. Analysis of video data revealed the amount of refractive shift, induced by the thermal lens, and the thermal relaxation as a function of time and power. Refractive shift data demonstrate that thermal lensing has a logarithmic transient response, but is linear with respect to power. Video analysis also shows the exponential decay of the thermal gradient. Confocal imaging of a water-filled artificial eye showed that for equivalent input powers, thermal lensing was strongest for the 1150nm wavelength followed by 1130, 1318 and 1110-nm. 


\subsection{INTRODUCTION}

In this chapter, a pump-probe dual-beam geometry is used in conjunction with a confocal imaging system and a high speed camera to observe the transient changes of the probe-beam's profile inside both vitreous and water media in an artificial eye. The location of a minimum beam waist is tracked through time and space in a video file to observe the rate of movement of the beam's waist along the optical axis. In the first portion of this study, data are presented for both vitreous and water for comparison of the behavior of the thermal lens between samples. This comparison is used to validate the second portion of this study; an investigation on the magnitude of the thermal lensing effect in a water-filled artificial eye over a wide range of near-infrared wavelengths and power levels. Portions of the data in this chapter have been reported (Vincelette, Oliver et al. 2009).

\subsection{BACKGROUND}

In vivo retinal damage studies are conducted largely on the Macaca mulatta (rhesus). As described in section 2.2.2, the percent of transmitted energy of laser radiation to the retina begins to drop sharply over the $1100-1150 \mathrm{~nm}$ wavelength region, remaining fairly flat from $1150-1300 \mathrm{~nm}$, then begins to plummet once more to nearly zero transmission at $1350 \mathrm{~nm}$ (see Fig. 3.1). In comparison, the artificial eye used in these experiments, called by name as the Cain cell, was created to simulate the optical configuration of the Rhesus' eye when filled with water (Cain, Noojin et al. 1997). The optical configuration of the Cain cell is given in Fig. 3.2. The percent of transmitted energy to the focal plane of a water-filled Cain cell was determined by applying Beer's 
law with linear absorption coefficients for water as $0.35,0.56,0.78$, and $1.34 \mathrm{~cm}^{-1}$ for 1110, 1130, 1150 and 1318-nm wavelengths respectively (see Fig. 3.1). An energy loss of $17 \%$ was measured experimentally for all the NIR wavelengths in an empty Cain-cell lens with two air-SF11 interfaces. Zemax software simulations of the SF11 Cain-cell lens resulted in Fresnel reflection losses of $\sim 8 \%$ for each air-SF-11 interface and $\sim 4 \%$ for a SF-11-water interface. Zemax results showed negligible linear absorption in the SF-11 glass for NIR wavelengths. As a result, the total computed losses from Fresnel reflections in a water-filled Cain-cell artificial eye are $\sim 12 \%$ for the NIR wavelengths in this study. The percent of transmitted energy, after an expected $12 \%$ loss, to the focal plane of the Cain cell is reasonably close to that of the predicted values for the rhesus eye (see Fig. 3.1b).

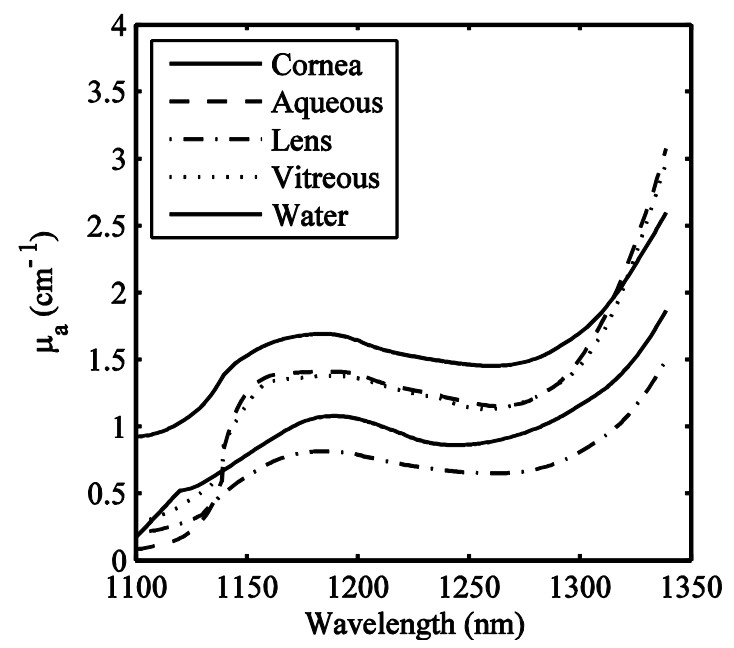

(a)

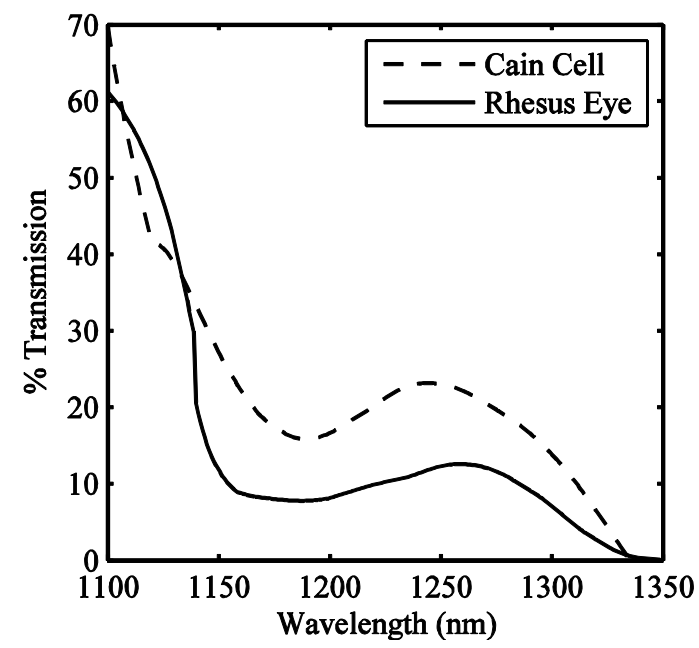

(b)

Figure 3.1: (a) The linear absorption coefficients for various media used to predict the percent transmission (Hale and Querry 1973; Maher 1978). (b) The percent of transmitted energy from the surface of the cornea to the retina for a rhesus eye (assuming no specular reflection) and the water-filled Cain cell (assuming 12\% loss in energy from the Cain-cell lens) (Vincelette, Rockwell et al. 2008). The average loss in the Cain cell lens was determined experimentally over a range of near-infrared wavelengths (see methods section). 
With water comprising the bulk of the vitreous fluid, vitreous' thermal properties are typically taken to be equal or very close to those of liquid water. Vitreous and water may share similar thermal properties, but upon visual observation it is evident that vitreous humor has a very different consistency. Vitreous is a Newtonian fluid, but due to the presence of collagen fibrils, vitreous cannot be readily suctioned into a thin syringe or segmented into parts without some effort to cut through the collagen fibrils (Lee, Litt et al. 1992). To date, the thermal lensing effect between water and vitreous has never been experimentally investigated.

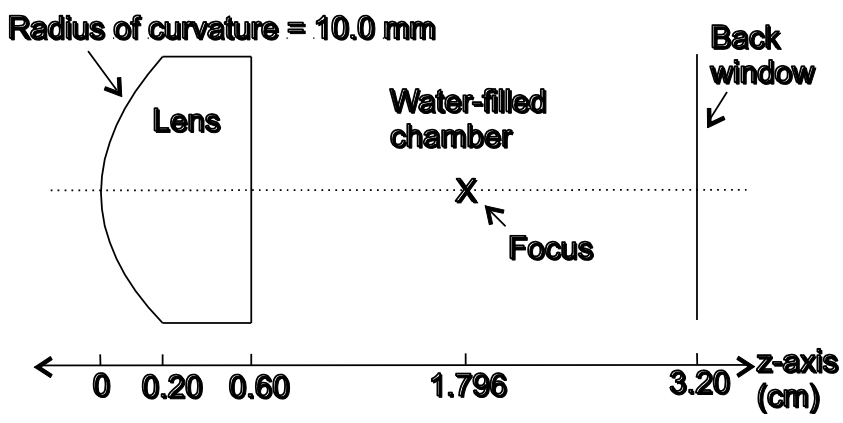

Figure 3.2: The optical configuration of the Cain-cell artificial eye (Cain, Noojin et al. 1997). The custom lens is made from SF11 glass. To create the curve in Fig. 3.1b for the Cain cell, 100\% energy was assumed at the surface of the lens, and a $\sim 12 \%$ loss was assumed through the lens (loss was determined using a Coherent power-meter in an empty Cain-cell and Zemax simulations). Finally Beer's law was applied to the waterfilled chamber to determine the transmitted energy at the focus.

The thermal lensing effect is usually exploited by z-scan spectroscopy to measure absorption, $\mu_{\mathrm{a}}$, or thermo-optic coefficients, dn/dT (Franko and Tran 1996; Vincelette, Thomas et al. 2009). Typically, z-scan spectroscopy utilizes a long Rayleigh range with a shorter sample pathlength. This condition of a longer Rayleigh range relative to the absorption sample pathlength cannot be met in an eye. In this study, we do not seek to measure the value of $\mathrm{dn} / \mathrm{dT}$ or $\mu_{\mathrm{a}}$, but rather seek to determine if the thermal lens is the same in water and vitreous in addition to quantifying when thermal lensing would 
become significant. Examination of the thermal lens in ocular media should include the use of a 2D detector, like that of a camera, to capture information about any asymmetry and non-linear effects such as the formation of a halo in the laser-beam's profile in the media. Unfortunately, placing a fast-frame rate camera at the focus would require the camera to withstand exposure to water and add the complication of size constraints to fit into an artificial eye chamber. Thus, in order to observe the thermal lens in an optical configuration similar to that of the eye, a new observational method was required.

A confocal imaging system allows for observation of the focused light at different spatial planes within the sample by using a pinhole to remove out of focus light and stepping the focal plane of the imaging system through the sample. For this study, the imaging system must house a long focal length objective to observe spatial positions inside the artificial eye. A pinhole is not used in this study since the laser spot size and shape contains important information pertaining to the behavior of the thermal lens. The imaging system can be referenced spatially by utilizing a pump-probe system such that the $\mathrm{z}=0$ position of the imaging system is determined by finding the probe-beam's minimum beam profile on the camera detector. The imaging system is then held stationary at the reference plane and the probe laser spot created at that focal plane is measured as a function of time (see Fig. 3.3). The imaging system can then be moved in small spatial increments along the z-axis with negative and positive values of $\mathrm{z}$ referring to focal positions in front and behind the reference plane respectively. With proper timing hardware, each exposure at each spatial step in the confocal imaging system is synchronized to a time, $\mathrm{t}=0$, reference. The resulting data; $\mathrm{x}, \mathrm{y}, \mathrm{z}$, time and intensity, are compiled into video files for observation of the shift of the thermal lensing effect through time and space. 
In this study, the IR pump laser alters the index of refraction in an artificial eye. The resulting transient lens induced by the pump laser causes the HeNe probe laser to behave according to the changes in the index of refraction. This chapter describes two related experiments. Both experiments used the same confocal imaging system, timing hardware, and data analysis. Different artificial eye configurations were used in this study since approximately $2-3 \mathrm{~mL}$ of fresh vitreous were available; not enough to fill the Cain-cell artificial eye, but suitable for a $10-\mathrm{mm}$ cuvette. The first experiment aimed to compare if thermal lensing induced by a continuous-wave, CW, 1313-nm laser radiation wavelength source in water and vitreous fluid were comparable. With the limited volumetric quantity of vitreous, $2-3 \mathrm{~mL}$, the artificial eye was constructed from a planoconvex 25.4-mm focal length lens and a 10-mm quartz cuvette. The 25.4-mm focal length lens and $10-\mathrm{mm}$ cuvette will be referred to as the modified artificial eye. By validating that water is a suitable substitute for vitreous humor in thermal lens investigations in the modified artificial eye, the first experiment mitigated the need to obtain $\sim 20$-mL of fresh vitreous fluid which would have been required to fill the Caincell artificial eye. The Cain-cell artificial eye is the preferred optical configuration for this investigation of thermal lensing since it is custom designed to mimic the optical geometry of the rhesus eye. The second experiment used a water-filled Cain-cell artificial eye exposed to CW 1110,1130, 1150 and 1318-nm wavelengths over a range of powers. 

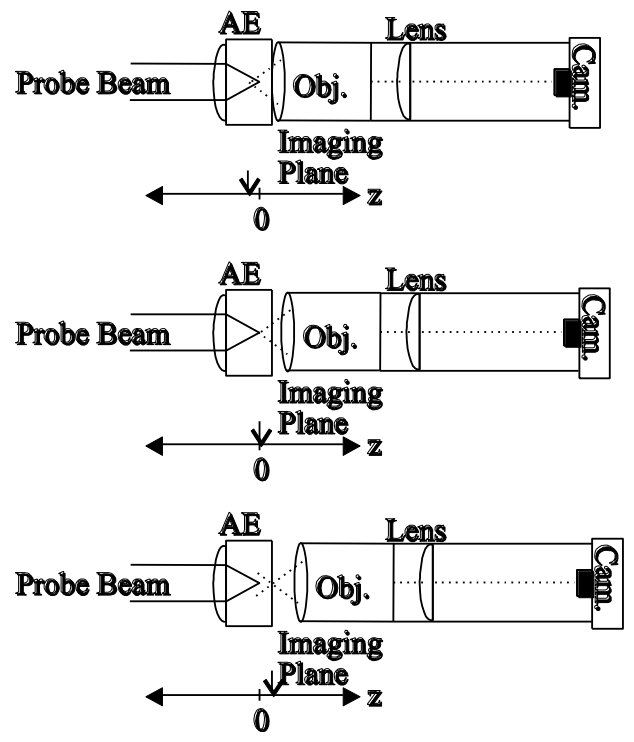

(a)

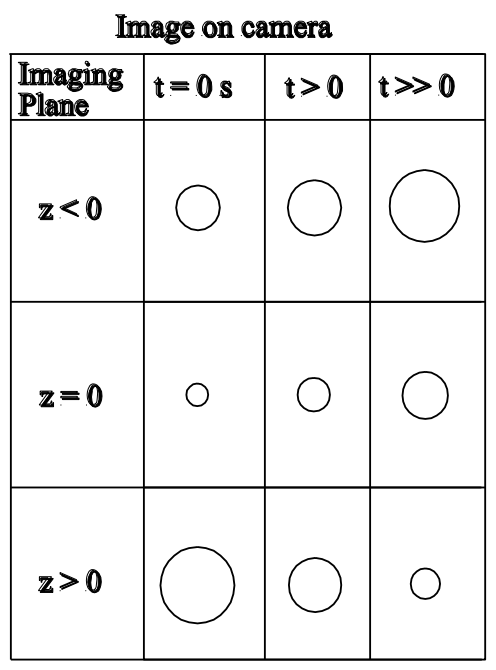

(b)

Figure 3.3: (a) The confocal imaging system at observational imaging planes before, at and after the probe-beam's focus, $\mathrm{z}=0$. $\mathrm{AE}$ is the artificial eye (composed of a short focal length lens and a sample holding chamber). Obj. is the microscope objective. Cam. is the camera. (b) The resulting transient information provided by exposures at each of the respective imaging planes in (a).

\subsection{MATERIALS AND METHODS}

\subsubsection{Thermal Lens Investigation Using a Modified Artificial Eye}

\subsubsection{Preparation and handling of the vitreous sample}

Based upon the availability of tissues, the eyes of adult Yucatan mini-pigs and Yorkshire pigs were used in this study. All tissues were collected through an approved tissue-sharing animal protocol approved by the Air Force Research Laboratory at Brooks City-Base. In all cases, the eyes were enucleated within twenty minutes and prepared for

experimentation within one hour post-mortem. After the eye was enucleated, it was trimmed of all excess tissue then rinsed with deionized water to assist with keeping blood 
and other cross-contaminants out of the vitreous. To remove the vitreous, an incision was made behind the limbus using a scalpel. Surgical scissors were used to continue the initial cut around the globe of the eye to separate the eye into two halves; one as a cup of tissue containing the cornea, aqueous and crystalline lens and the other as a cup containing the vitreous and retina. A disposable polyethylene pipette was cut in half to allow for a larger surface area for gentle suction to pull the vitreous carefully away from the retinal cup. If proper care was not taken in this step, pigmented tissues from the retina and/or iris linkages became extensively mixed into the vitreous sample, rendering the sample unusable. With proper, gentle suction, the vitreous pulled away from the retina with minimal pigmented strands of tissue. The vitreous was then transferred into a 10-mm quartz cuvette and held at room temperature for the remainder of the experiment. To minimize any risk of tissue degradation, the vitreous was never kept longer than four hours post-mortem.

To investigate if near-infrared laser radiation may be capable of coagulating the vitreous, a vitreous sample was kept inside a $10-\mathrm{mm}$ quartz cuvette and placed at the focus of a 25.4-mm focusing lens in front of a high power $(\sim 770-\mathrm{mW}) 1318-\mathrm{nm} \mathrm{CW}$ laser source. (Note - this laser source was only available for this specific portion of the experiment.) The vitreous sample was allowed to remain in place for 5 minutes to observe for coagulation. A small thermo-couple near the NIR beam-path (confirmed not to be directly in the beam path) was used to observe the approximate temperature rise inside the vitreous sample.

The density of a fresh vitreous sample, not used in the optical system, was measured. A 10-mL glass graduated cylinder was placed on a digital scale and zeroed. The mass of $3-\mathrm{mL}$ of deionized water was then measured by pouring it in the graduated 
cylinder and used as a standard. The mass and volumetric displacement were recorded when a sample of vitreous was added to the graduated cylinder containing the water.

\subsubsection{Optical configuration}

The pump-beam, a 1313-nm IRCL-150-1313-P-L CrystaLaser (S/N 24060101679), was collimated and aligned to create a $1 / \mathrm{e}^{2}$ beam diameter at the focusing lens of $\sim 3.25 \mathrm{~mm}$ and an $\mathrm{m}^{2}$ of 1.16 . The power of the 1313-nm laser was attenuated by using a Thorlab NEK01 neutral density (ND) filter set, with a maximum power of 129-mW delivered to the sample cuvette. The probe-beam, a Melles Griot 5-mW 633-nm HeNe laser was collimated and aligned to produce a $1 / \mathrm{e}^{2}$ beam diameter of $\sim 3.65 \mathrm{~mm}$. See Fig. 3.4 for the overall optical set-up. The two beams were combined by using a dichroic mirror, CVI LWP-0-R633-T1319-PW-1025-C, which transmitted the pump and reflected the HeNe probe laser radiation. To mimic the eye, a Thorlabs 25.4-mm BK7 planoconvex lens was used as the focusing lens to send the laser radiation through a 10$\mathrm{mm}$ quartz cuvette containing deionized water or vitreous from an adult Yucatan minipig. The sample cuvette was held on a micrometer stage which could move along the optical axis to place the sample at controlled locations in the optical path. The imaging system was placed in the optical path and coupled to another micrometer stage to allow for movement of the imaging system independently of the remaining optical train, see Fig. 3.4. 


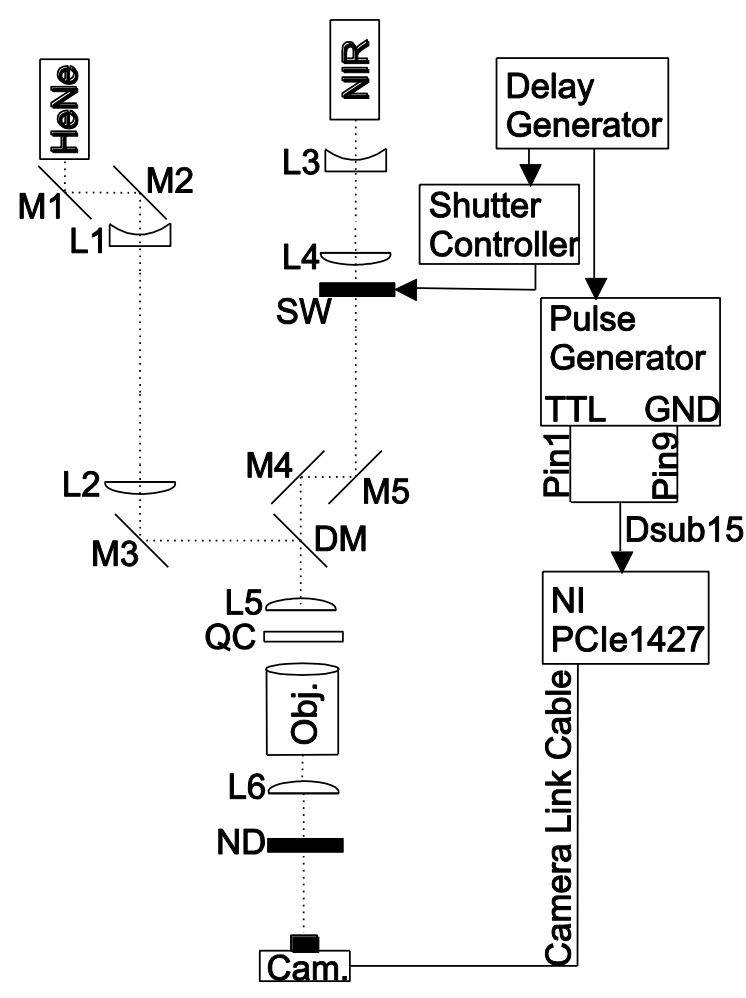

Figure 3.4: The optical set up using the modified artificial eye. M1-3: Broadband-visible mirrors. M4 and M5: Near-infrared mirrors. L1-5: Thorlabs lenses with -100, 500, -100, 200 and 25.4-mm focal lengths respectively. SW: 10-mm diameter electronic shutter. DM: Dichroic mirror made (CVI LWP-0-R633-T1319-PW-1025-C) transmitted the pump and reflected the probe laser. QC: Quartz cuvette to hold the sample. The imaging system consisted of a Mitutoyo objective (Obj.), a Newport optics 200-mm lens (L6), neutral density filters (ND) and a Photon Focus camera (cam., model MV-D1024-160CL-8). Delay generator: Stanford Research Systems 4-Channel Digital Delay Generator, model DG-535. Shutter controller: Uniblitz SD-10 Shutter Driver Timer. Pulse generator: HP8116A Pulse/Function Generator. The National Instruments framegrabbing card was a NI PCI-e1427 and installed on a Dell Dimension E521 (Model DC6M) with an AMD 64 Athlon X2 Processor running Windows XP. The imaging system is moved along the optical axis to observe individual positions in $\mathrm{Z}$-space relative to the back of the sample cuvette through time at a high frame-rate.

All experiments were run at room temperatures between $20-23{ }^{\circ} \mathrm{C}$. Careful detail was taken to attempt to place the cuvette such that the probe-beam's minimum waist was delivered to the inner back surface of the quartz cuvette. A summary of the experimental data matrix for this portion of the study is given in Table 3.1 . 
Table 3.1: A summary of the experimental data matrix for the optical configuration depicted in Fig. 3.4. $\Delta \mathrm{z}$ describes the position of the confocal system's imaging plane relative to the initial-state focus at $\mathrm{t}=0$ with negative and positive values denoting imaging planes in front of and behind the initial-state focus respectively. Power is reported as the power reaching the front surface of the quartz-cuvette.

$$
\begin{gathered}
1 / \mathrm{e}^{2} \text { Diameter at focusing } \\
\text { lens }(\mathrm{mm})
\end{gathered}
$$

Probe Beam : $\quad 3.65$

\begin{tabular}{|c|c|c|c|c|c|}
\hline \multirow[t]{2}{*}{ Sample } & \multirow{2}{*}{$\underset{(\mathrm{nm})}{\operatorname{Pump} \lambda}$} & \multirow{2}{*}{$\begin{array}{l}\text { Power } \\
(\mathrm{mW})\end{array}$} & \multicolumn{2}{|c|}{$\begin{array}{l}\text { Range of } \Delta z \\
\quad(\mu \mathrm{m})\end{array}$} & \multirow{2}{*}{$\begin{array}{c}\text { Exposure } \\
\text { Duration } \\
\text { (s) }\end{array}$} \\
\hline & & & Start & End & \\
\hline Vitreous & \multirow{3}{*}{1313} & 46 & 254 & 762 & \multirow{3}{*}{1} \\
\hline Vitreous & & 129 & -234 & 1143 & \\
\hline Water & & 129 & -127 & 1143 & \\
\hline
\end{tabular}

Pump Beam : $\quad 3.25$

\subsubsection{Thermal Lens Investigation Using the Cain-Cell Artificial Eye}

The choice of wavelengths utilized in this experiment was 1110, 1130, 1150 and $1318 \mathrm{~nm}$ due to these wavelengths' variation on the curve depicted in Fig. 3.1b. The transmissions associated with these wavelengths in the artificial eye were 54.2, 39.0, 27.5, and $8.1 \%$ respectively. A Coherent Mira-OPO laser pumped by a Coherent Chameleon was tuned to a selected wavelength, with a full-width half-maximum (FWHM) of 12-15 nm, then aligned into the system before starting each set of data collection. Once the OPO was tuned, deviation from the selected central wavelength was no greater than $1 \mathrm{~nm}$. The experimental set up is shown in Fig. 3.5. A pump-probe geometry was used with the Mira-OPO laser-radiation wavelength acting as the pump and a 633-nm Melles Griot HeNe laser acting as the probe. The pump and probe lasers were co-aligned and sent through the deionized water-filled Cain cell serving as the artificial eye $(\mathrm{AE})$. The water was at room temperature $\left(\sim 20^{\circ} \mathrm{C}\right)$ for all experiments. The 
probe laser was expanded and aligned to create a $1 / \mathrm{e}^{2}$ beam diameter of $\sim 3.32 \mathrm{~mm}$ at the front surface of the Cain-cell lens as determined by a knife edge measurement. The power of the probe laser was less than $1.8 \mathrm{~mW}$ and remained exposed to the sample throughout the experiments. A waveplate (WP) placed in front of the OPO laser-head was used to control the amount of power delivered to the sample plane. The power of the OPO laser radiation was checked at the sample plane before and after each data collection set to ensure the stability of the laser. The OPO-laser power never deviated by more than +/- $5 \mathrm{~mW}$ over several hours time.

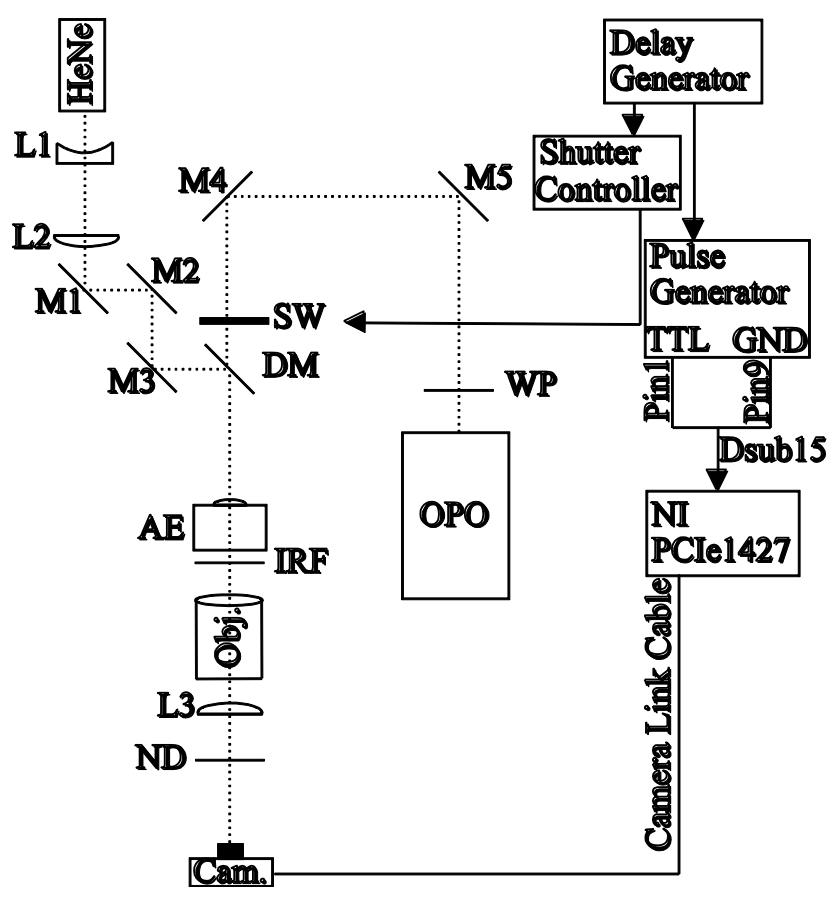

Figure 3.5: The experimental set up using Cain-cell artificial eye. L1-3 were -10, 50 and $20 \mathrm{~cm}$ focal length Thorlab BK7 glass lenses respectively. M1-3 were broad-band visible mirrors while M4 \& 5 were silver coated mirrors. WP was the waveplate to control the power. SW was the Uniblitz electronic shutter. DM was a dichroic mirror to transmit in the NIR and reflect in the visible to co-alignment the beams. AE was the artificial eye. IRF was an infrared filter to protect the camera from the high-power NIR exposure. The long working distance $(3.3 \mathrm{~cm})$ microscope objective $(\mathrm{Obj}$.), lens element L6 and camera (Cam.) comprised the confocal imaging system and were mounted together on a micrometer stage. ND was the neutral density filter to control saturation of the time $=0$ image at $\mathrm{z}=0$. 
Data were obtained for at least three power settings at each wavelength (Table 3.2). When possible a fourth power was used which was the maximum output of the selected wavelength (this was not possible for the 1318-nm wavelength since $450 \mathrm{~mW}$ was the maximum available). A Coherent PM3 Powermeter was used to measure the power transmitted through an empty Cain cell for each NIR wavelength. These power measurements determined the empty Cain-cell lens has an average loss of $17 \%$ when aligned in the optical path. The 17\% loss does not account for the index change of SF11 glass to water. Zemax was used to estimate the losses of an air-SF11 interface and SF11water interface, resulting in $\sim 8 \%$ and $4 \%$ loss from Fresnel reflections respectively. Given the computed results from Zemax, I would expect to get $\sim 16 \%$ loss for an empty Cain-cell (measured $\sim 17 \%$ ) and $\sim 12 \%$ for a water-filled Cain cell. NIR detectors are costly and cannot be placed into the water-filled Cain cell, the percentage of Fresnel loss from the SF11 lens is taken to be the computed value of $12 \%$. 
Table 3.2: A summary of the experimental data matrices for the optical configuration in Fig. 3.5. Power is reported as the power reaching the front surface of the Cain-cell lens. Based on results from Fig. 3.1b, the power reaching the focal position for the NIR laser radiation is approximately $54.2,39.0,27.5$, and $8.1 \%$ of the power input at the front of the Cain cell lens for $1110,1130,1150$ and 1318-nm wavelengths respectively (the percentages do account for an approximate $12 \%$ loss in the lens: loss based upon measurements and simulations for an empty and water-filled cell).

\begin{tabular}{cc} 
& $\begin{array}{c}1 / \mathrm{e}^{2} \text { Diameter at Cain-cell } \\
\text { lens }(\mathrm{mm})\end{array}$ \\
\cline { 2 - 2 } Probe Beam : & 3.32 \\
Pump Beam : & 3.95
\end{tabular}

\begin{tabular}{|c|c|c|c|c|}
\hline \multirow{2}{*}{$\begin{array}{l}\text { Pump } \\
\lambda(\mathrm{nm})\end{array}$} & \multirow{2}{*}{$\begin{array}{l}\text { Power } \\
(\mathrm{mW})\end{array}$} & \multicolumn{2}{|c|}{$\begin{array}{c}\text { Range of } \Delta \mathrm{z} \\
(\mu \mathrm{m})\end{array}$} & \multirow{2}{*}{$\begin{array}{c}\text { Exposure } \\
\text { Duration } \\
\text { (s) }\end{array}$} \\
\hline & & Start & End* & \\
\hline \multirow{4}{*}{1110} & 890 & \multirow{8}{*}{-50} & 600 & \multirow{8}{*}{1} \\
\hline & 450 & & 300 & \\
\hline & 300 & & 250 & \\
\hline & 150 & & 150 & \\
\hline \multirow{4}{*}{1130} & 790 & & 650 & \\
\hline & 450 & & 400 & \\
\hline & 300 & & 300 & \\
\hline & 150 & & 150 & \\
\hline
\end{tabular}

\begin{tabular}{|c|c|c|c|c|}
\hline \multirow{2}{*}{$\begin{array}{l}\text { Pump } \\
\lambda(\mathrm{nm})\end{array}$} & \multirow{2}{*}{$\begin{array}{l}\text { Power } \\
(\mathrm{mW})\end{array}$} & \multicolumn{2}{|c|}{$\begin{array}{l}\text { Range of } \Delta z \\
\qquad(\mu \mathrm{m})\end{array}$} & \multirow{2}{*}{$\begin{array}{l}\text { Exposure } \\
\text { Duration } \\
\quad \text { (s) }\end{array}$} \\
\hline & & Start & End* & \\
\hline \multirow{4}{*}{1150} & 735 & \multirow{7}{*}{-50} & 700 & \multirow{7}{*}{1} \\
\hline & 450 & & 500 & \\
\hline & 300 & & 400 & \\
\hline & 150 & & 250 & \\
\hline \multirow{3}{*}{1318} & 450 & & 400 & \\
\hline & 300 & & 250 & \\
\hline & 150 & & 200 & \\
\hline
\end{tabular}

* - End is defined as the last z-position of the imaging system relative to the initial-state focal position.

\subsubsection{Timing Hardware, Data Acquisition and Analysis}

All thermal lensing experiments in this study used the same timing hardware, data acquisition and analysis. A Uniblitz SD-10 shutter driver (S/N 85075) was used to control a 10-mm shutter for the delivery of the pump laser beam to the sample. The probe beam was allowed to remain on at all times before, during and after the delivery of the pump laser exposure. A Stanford Research System Delay Generator (Model DG-535, $\mathrm{S} / \mathrm{N}$ 07148) was used to synchronize the shutter driver with image acquisition. The delay generator was set to a user-controlled push-button entry mode. Upon execution, the 
delay generator triggered a pulse sequence on a HP 8116A Pulse/Function Generator (S/N 3134 G 18809) which acted as the driving pulse for each individual frame captured in the image acquisition sequence. Pins 1 and 9 of a DSUB15 cable were connected to the TTL and ground of the pulse generator respectively to communicate with the image acquisition card (NI PCI-e1427). A Camera Link ${ }_{\circledast}$ cable connected the camera to the image acquisition card. StreamPix 3 software, created by Norpix, was used to stream the images to disk at a fast frame rate, $150 \mathrm{fps}$, as dictated by the TTL pulse from the pulse generator. All image post-processing was performed using $\mathrm{MATLAB}_{\circledast}$ and/or ImageJ.

Each exposure sequence acquired 3.5 seconds of data, to observe the excitation and relaxation of the sample from the 1-sec exposure from the near-infrared radiation source. Each imaging sequence corresponded to a specific z-position of the imaging system relative to the minimum beam waist position. With the probe laser on and pump laser blocked, the imaging system was moved along the z-axis until the smallest beamprofile was observed; this was the $\mathrm{z}=0$ position. When the imaging system is said to be at $\mathrm{z}$-positions $<0$ and z-positions $>0$ the system was imaging beam-profiles in front and behind the initial-state focus respectively. Combing a sequence of exposures over several z-positions allows for five-dimensions of data; $x, y, z$, time, and intensity.

A room-light noise frame was subtracted from the 8-bit grayscale data frames. Images were then filtered with a median filter (window size of 5) and full-scale contrast stretched in Matlab. Video files consisting of the synchronized space and time slices were compiled to allow for side-by-side observation of experimental data and allowed for tracking the approximate position of the minimum beam-waist. The position of the minimum beam-waist was then plotted against time to quantify the rate and magnitude of thermal lensing for the pump-beam laser radiation's power and wavelength. 


\subsubsection{Reduction of Multidimensional Data}

Data in this study contained information on the thermal lens in relation to time, power, wavelength, $\mathrm{x}, \mathrm{y}$, and $\mathrm{z}$ space and changes in intensity. Reducing the complexity of the data to quantify the strength of the thermal lens in terms of just four dimensions (time, power, wavelength, and z-position) was chosen. Basic observations are made on the results in regards to $\mathrm{x}, \mathrm{y}$ and intensity.

In the experiment depicted in Fig. 3.4, the optical configuration is not an optimal representation of a rhesus eye, so transient data are reported as $\Delta z$, the spatial-step of the confocal system relative to the initial-focus, as a function of time. This analysis is suitable to compare and contrast the thermal lens between vitreous and water media.

In the Cain-cell experiment depicted in Fig. 3.5, the optical configuration is designed for optimal conditions simulating a rhesus eye. Since refractive error discussions about the eye are typically in terms of Diopters (see section 2.2.3), in the Cain-cell experiment (Fig. 3.5), the transient response of the probe beam is reported as the refractive error, $\mathrm{R}$, induced by the thermal lens given as

$$
R=\left(1 / f_{0}\right)-\left(1 / f_{t}\right),
$$

where $f_{0}$ is the focal length of the Cain-cell lens, 17.96-mm (Fig. 3.2), and $f_{t}$ is the new focal length found in the experiment at time, $\mathrm{t}$ such that $\mathrm{f}_{\mathrm{t}}=\mathrm{f}_{0}+\Delta \mathrm{z}$, where $\Delta \mathrm{z}$ is again the spatial step of the confocal system relative to the focus at initial-state.

\subsection{RESULTS}

\subsubsection{Thermal Lensing of Vitreous and Water in the Modified Artificial Eye}

A sample of the video frames compiled from the experimental data collection for the data matrix given in Table 3.1 at initial-state (time $=0$ ), steady-state (time $=1 \mathrm{~s}$ ), and 
time points along the thermal relaxation from the 1-s exposure to the 1313-nm pumpbeam are given in Fig. 3.6. Notice the side-by-side comparison of the thermal lens between water and vitreous samples exposed under identical conditions. Analysis of the video-data allowed for tracking the approximate position of the minimum-beam waist through space and time. Analysis of the video-data in Fig. 3.6 is given in Fig. 3.7.

The error bars used in Fig.'s 3.7 and 3.8 were taken to be the spatial step between a z-position data point and its neighboring data point. For example, if the $\Delta \mathrm{z}$ position of a data point is $127 \mu \mathrm{m}$, its neighboring data points are $0 \mu \mathrm{m}$ and $254 \mu \mathrm{m}$, making the 127- $\mu \mathrm{m}$ data point's error bars would be $+/-127 \mu \mathrm{m}$. 


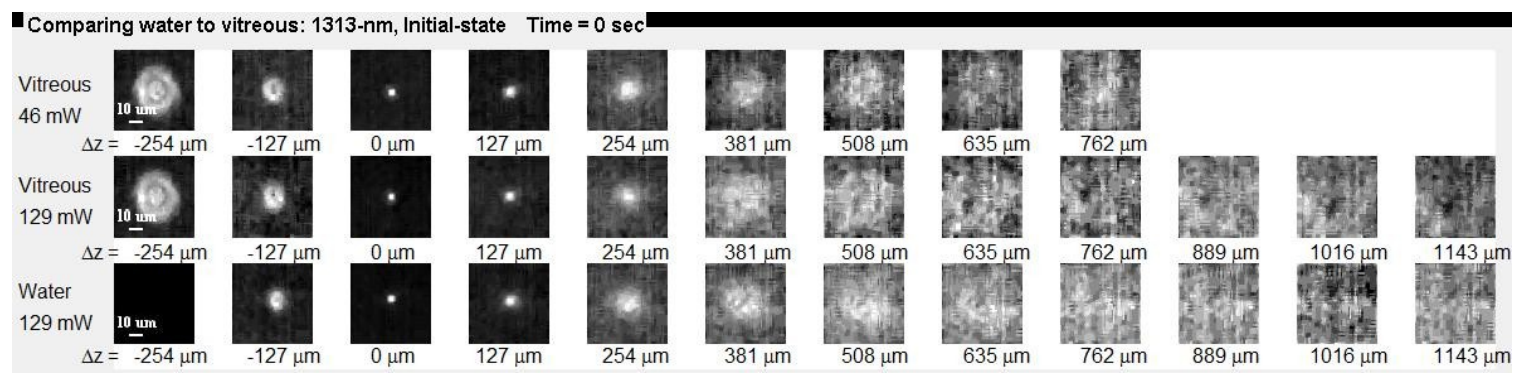

(a)

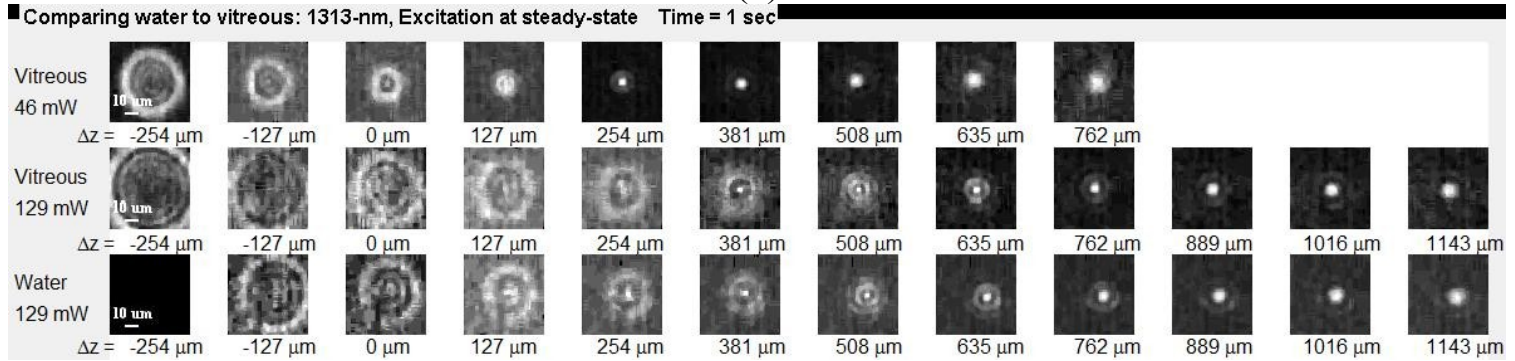

(b)

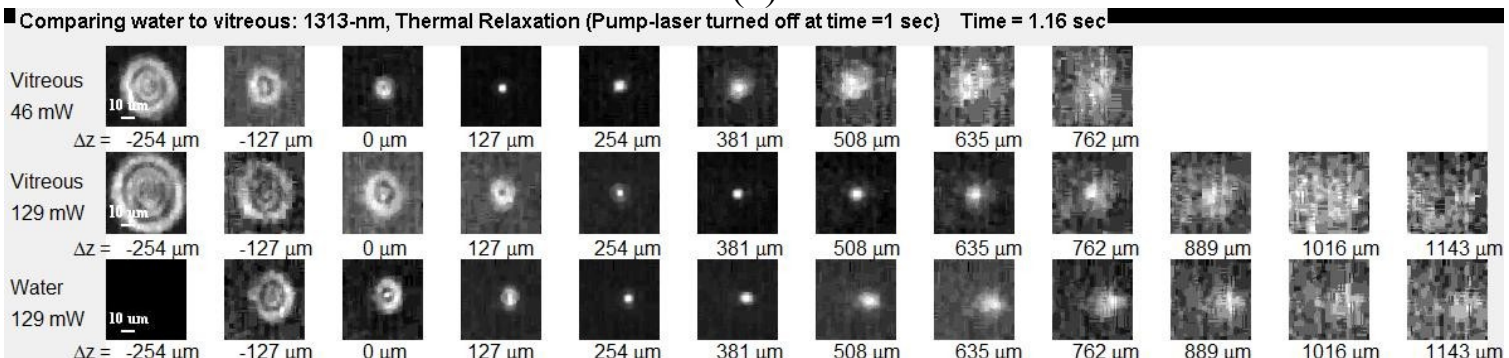

(c)

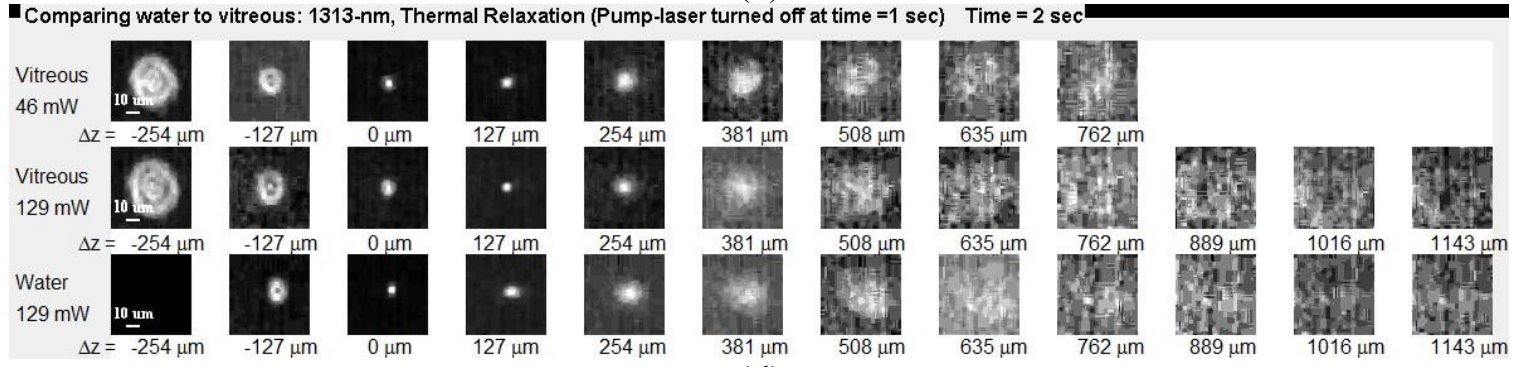

(d)

Figure 3.6: Some examples of the resulting video-frames compiled from the data analysis of vitreous and water samples in the modified artificial eye at (a) resting/initialstate, (b) at steady-state (time $=1 \mathrm{~s}$ ), (c) at $0.16 \mathrm{~s}$ after the pump-laser is turned off and (d) $1 \mathrm{~s}$ after the pump-laser is turned off. 


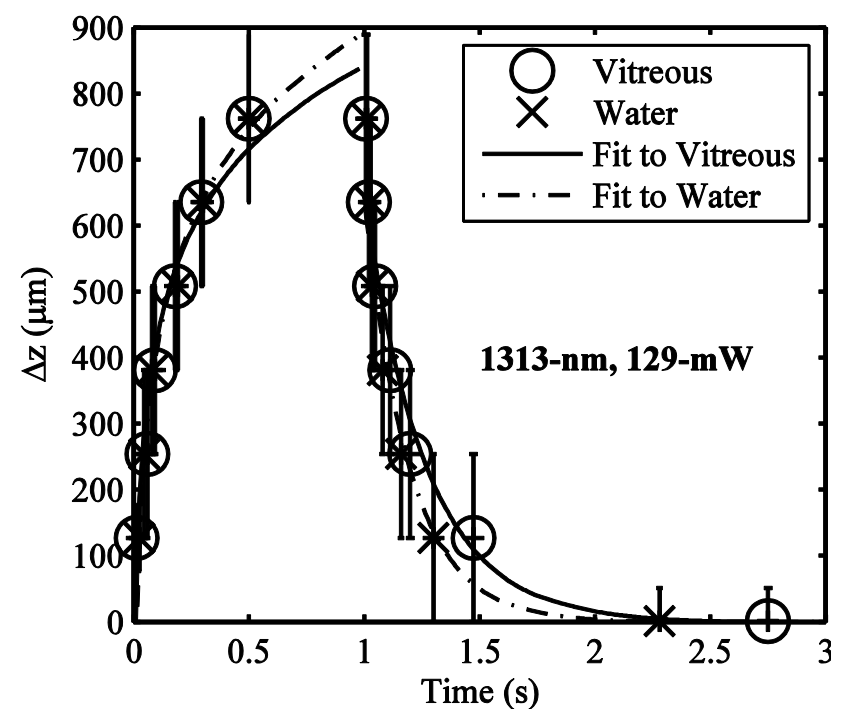

Figure 3.7: Comparison of the rates of the thermal lens induced in the vitreous and water samples exposed to $129-\mathrm{mW}$ of $\mathrm{CW}$ laser-radiation at a wavelength of $1313-\mathrm{nm}$ for $1-\mathrm{s}$. Experimental data presented in this figure are the result of analyzing the video results as given in the example frames in Fig 3.6. $\Delta z$ represents the focal plane of the confocal imaging system relative to the initial-state focus found at time $=0$. Error bars were previously explained in the text.

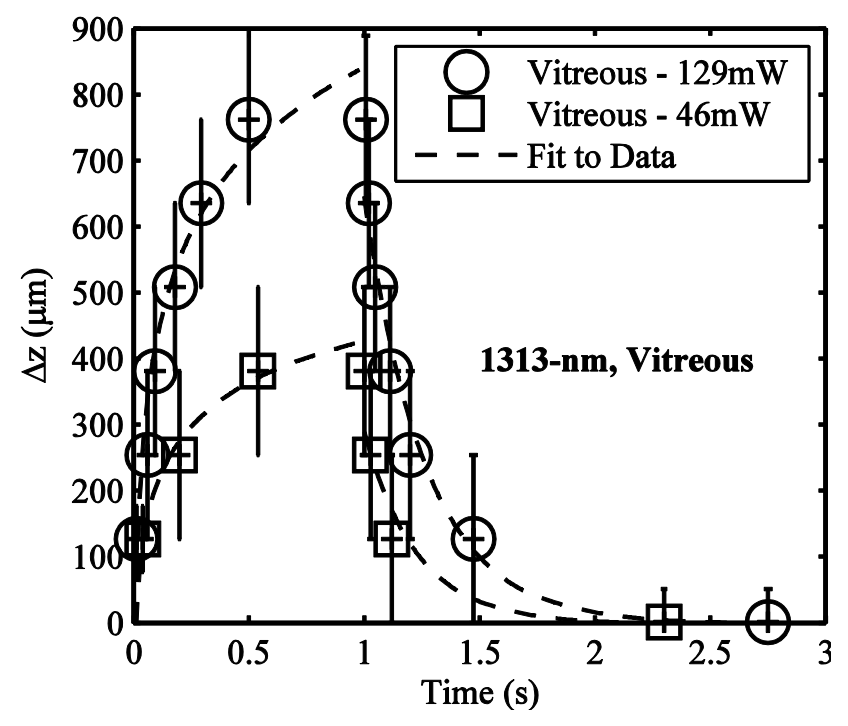

Figure 3.8: Comparison of the rates of the thermal lens induced in the vitreous sample exposed to two different power levels of CW laser-radiation at 1313-nm for $1 \mathrm{~s}$. 
Using a graduated cylinder, 3-mL of deionized $\mathrm{H}_{2} \mathrm{O}$ were measured out and weighed. The density of the $3-\mathrm{mL}$ of water was determined to be $0.9673 \mathrm{~g}$. A fresh vitreous sample was added to the $3-\mathrm{mL}$ of water in the graduated cylinder. The density of the vitreous sample was determined to be $1.0149 \mathrm{~g} / \mathrm{mL}$.

A fresh vitreous sample held in a quartz cuvette was placed at the focus of a 25.4$\mathrm{mm}$ focusing lens in front of a high power $(\sim 770-\mathrm{mW}) 1318-\mathrm{nm} \mathrm{CW}$ laser source. (Note - this laser source was only available for this portion of the experiment.) The vitreous sample was allowed to remain in place for 5 minutes to observe for morphological changes. No whitening (indicating protein denaturation) of vitreous was observed throughout 5 minutes of continuous exposure. A thermo-couple probe was used to record the approximate temperature rise inside the vitreous, near the beam-waist. Initially, the vitreous was at room temperature; $23.6^{\circ} \mathrm{C}$. After $\sim 10$-s, the probe read $29.1{ }^{\circ} \mathrm{C}$ and after $\sim 5$-min, the probe read $50.1^{\circ} \mathrm{C}$. At the end of 5-min, the entire cuvette was warm to the touch, but there was no visible whitening of the vitreous along or near the path of the laser beam. Further analysis of these data are reserved for the discussion.

\subsubsection{Thermal Lensing in the Water-Filled Cain-Cell Artificial Eye}

A sample of the video frames compiled from the experimental data collection is given in Fig. 3.5 for time, $\mathrm{t}=0$ and $1 \mathrm{~s}$, for the excitation exposure, respectively. A complete compilation of the experimental data matrix for this segment of the study is given in Table 3.2 . 
Water: $150 \mathrm{~mW}$, Initial-state Time $=0 \mathrm{sec}$

$1318 \mathrm{~nm}$
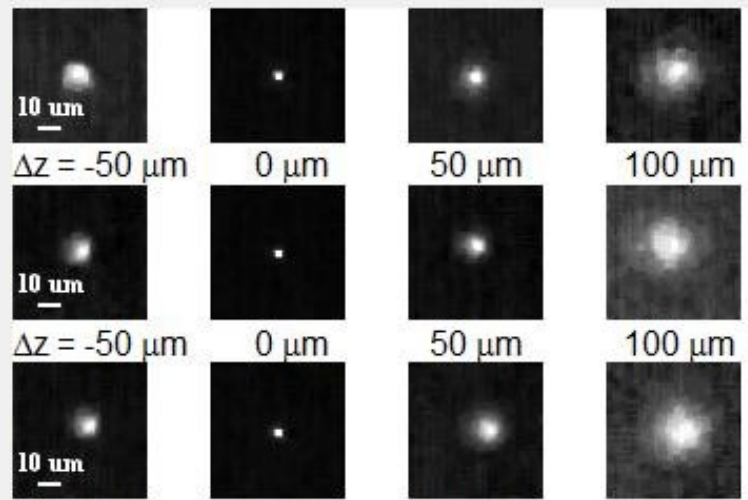

$100 \mu \mathrm{m}$

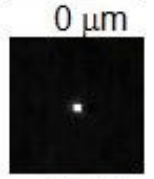

$\underline{10}=-50 \mu \mathrm{m}$

$0 \mu \mathrm{m}$
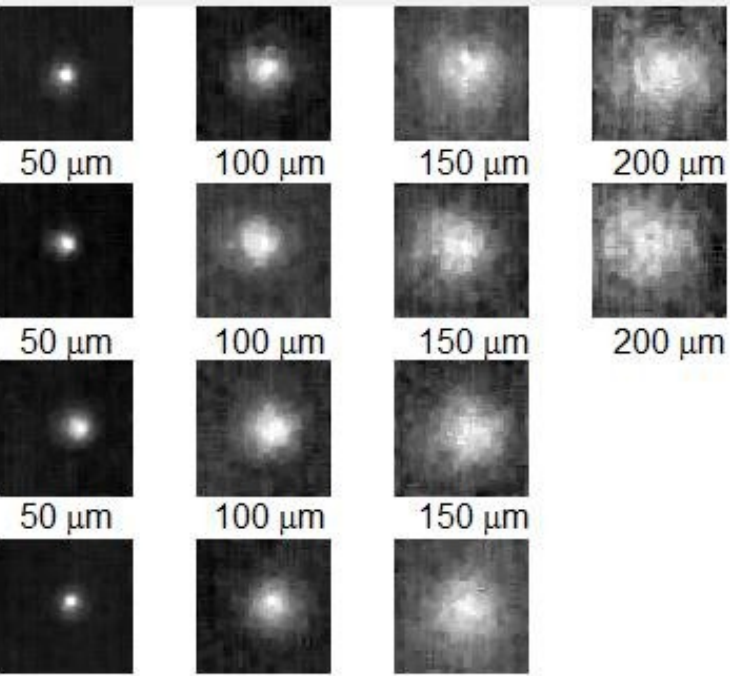

$150 \mu \mathrm{m}$

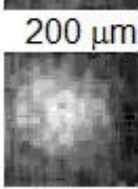

$200 \mu \mathrm{m}$

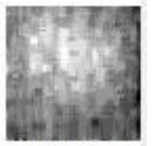

$150 \mu \mathrm{m}$

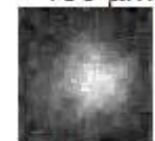

$250 \mu \mathrm{m}$

$1110 \mathrm{~nm}$
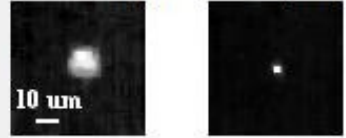

$\Delta \mathrm{Z}=-50 \mu \mathrm{m}$

$0 \mu \mathrm{m}$

$50 \mu \mathrm{m}$

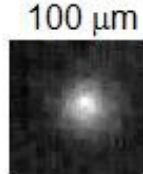

$150 \mu \mathrm{m}$

$100 \mu \mathrm{m}$

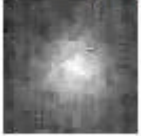

$150 \mu \mathrm{m}$

(a)

Water: $150 \mathrm{~mW}$, Steady-state Time $=1 \mathrm{sec}$

$1318 \mathrm{~nm}$

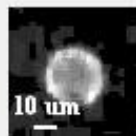

$\Delta \mathrm{z}=-50 \mu \mathrm{m}$

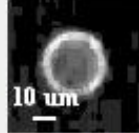

$\Delta \mathrm{z}=-50 \mathrm{um}$
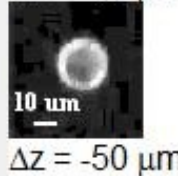

$1110 \mathrm{~nm}$

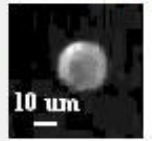

$\Delta \mathrm{Z}=-50 \mu \mathrm{m}$

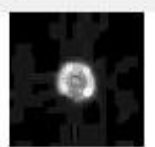

$0 \mu \mathrm{m}$
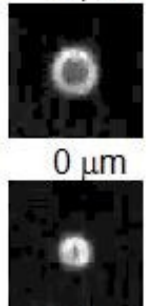

$0 \mu \mathrm{m}$

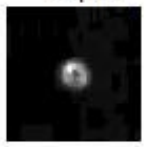

$0 \mu \mathrm{m}$
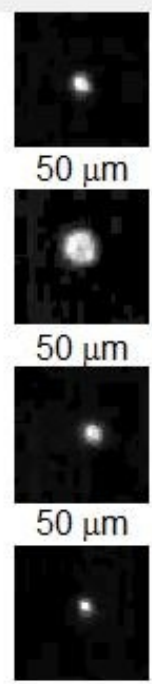

$50 \mu \mathrm{m}$

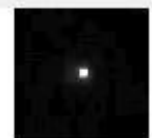

$100 \mu \mathrm{m}$
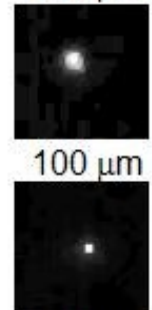

$100 \mu \mathrm{m}$

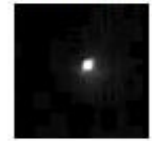

$100 \mu \mathrm{m}$

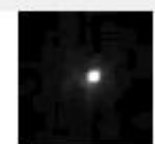

$150 \mu \mathrm{m}$

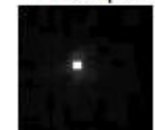

$150 \mu \mathrm{m}$

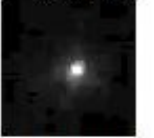

$150 \mu \mathrm{m}$

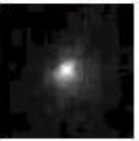

$150 \mu \mathrm{m}$

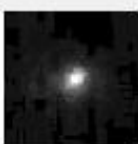

$200 \mu \mathrm{m}$

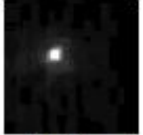

$200 \mu \mathrm{m}$

$250 \mu \mathrm{m}$

(b)

Figure 3.9: An example of the video frames which were compiled for analyzing the data sets for all four laser-radiation wavelengths at time, $t=0$ and $1 \mathrm{~s}$ of the 1 -s excitation exposure to $150-\mathrm{mW}$ of power input at the Cain-cell lens. (a) The initial state and (b) shows the steady-state. The value of the z-labels identifies where the confocal imaging system was placed relative to the probe-beam's initial focus at $t=0$. The error associated with the time slice is $+/-0.00667 \mathrm{~s}$. Differences in the thermal lensing effect between wavelengths are reserved for the discussion. 
Though the 1318-nm wavelength has an absorption coefficient, $\mu_{\mathrm{a}}$, of $1.34 \mathrm{~cm}^{-1}$, approximately only $8.1 \%$ of the energy is transmitted $(\% \mathrm{~T})$ to the focus compared to the 1150-nm wavelength which has a $\mu_{\mathrm{a}}$ of $0.78 \mathrm{~cm}^{-1}$ and a $\% \mathrm{~T}$ of $27.5 \%$ (see Fig. 3.1).

Video files were carefully analyzed to track the movement of the probe-beam's minimum waist position as a function of time to create the plots in Fig. 3.6. The $\Delta z(t)$ data were then converted into R(t) using Eq. 3.1 then plotted in Matlab. These data ( Fig. 3.10) show the excitation $(0<\mathrm{t}<1 \mathrm{~s})$ and relaxation $(1 \mathrm{~s} \leq \mathrm{t} \leq 3 \mathrm{~s})$ of the thermal lens induced by the pump-laser radiation wavelengths of $1110,1130,1150$ and $1318-\mathrm{nm}$. The legends in each graph in Fig. 3.10 identify the power of the pump-laser delivered to the front surface of the Cain-cell lens. The dashed lines in Fig. 3.10 are trendline fits provided by Excel. The error bars were set to $+/-$ the distance between the data point and its nearest neighbor on the vertical axis. 


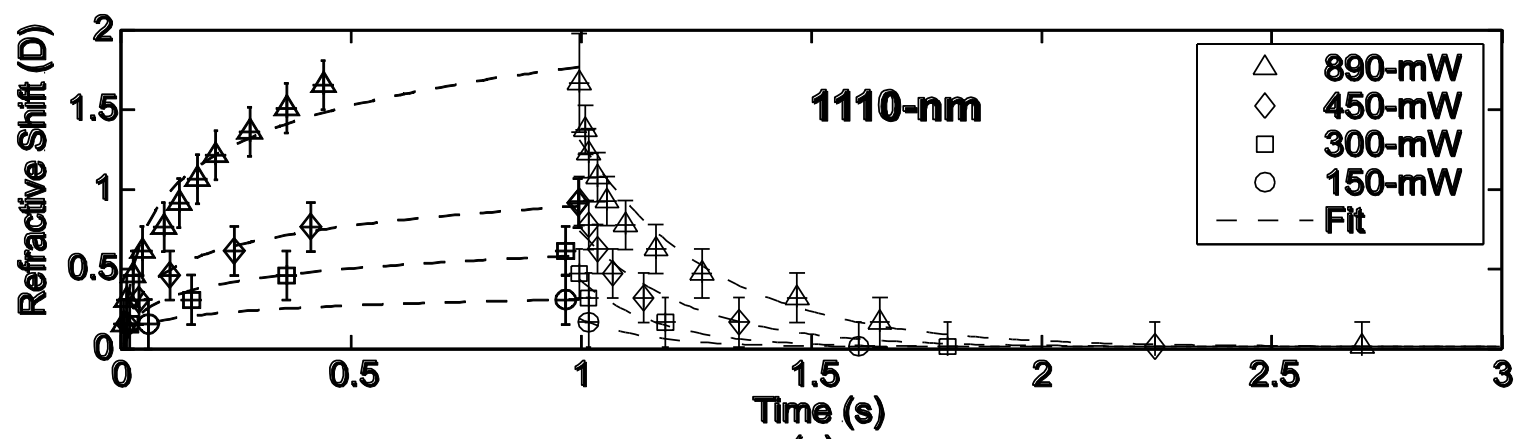

(a)

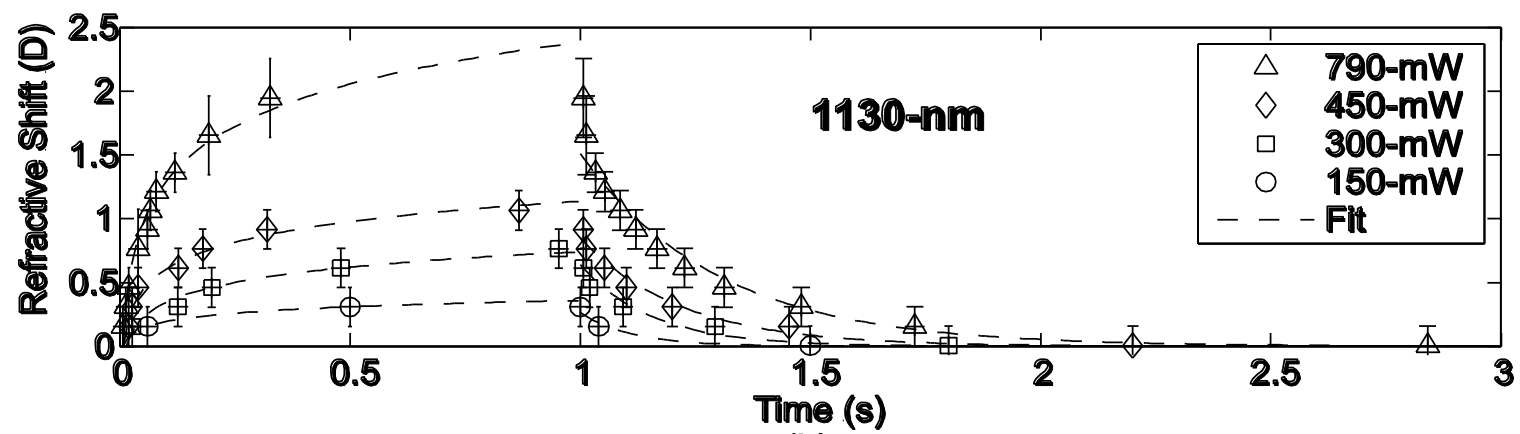

(b)

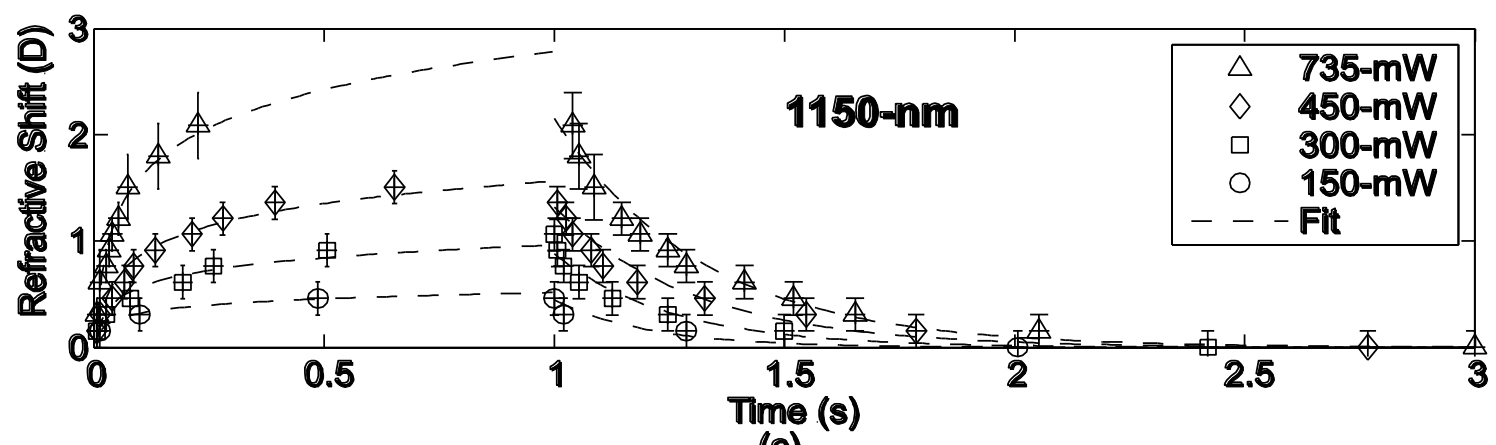

(c)

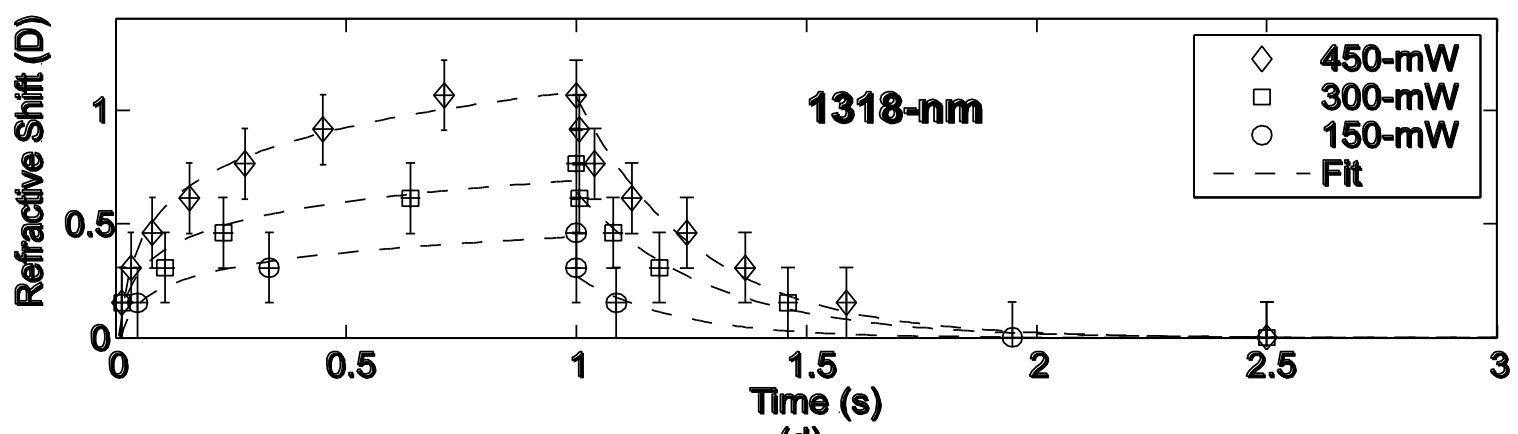

(d)

Figure 3.10: The excitation and relaxation of the thermal lens from the pump-laser radiation wavelengths of (a) 1110, (b) 1130, (c) 1150 and (d) $1318 \mathrm{~nm}$. 
The excitation data, for time $=0$ to $1 \mathrm{~s}$, in Fig. 3.10 were fit to a logarithmic function in Excel with the general form

$$
\mathrm{R}=\mathrm{A} \ln (\mathrm{t})+\mathrm{B} .
$$

$\mathrm{R}$ is the refractive error [D] and $\mathrm{t}$ is time $[\mathrm{s}]$. The thermal relaxation data, for time $=1$ to 3 seconds, in Fig. 3.10, were fit to an exponential decay in Excel with the general form

$$
\mathrm{R}=\mathrm{E}^{*} \exp (\mathrm{Ft}) .
$$

The values for the A, B, E and F coefficients for each data set are given in Table 3.3.

Table 3.3: The coefficients for the fits provided in Fig. 3.10. The coefficients A, B, E and $\mathrm{F}$ are described in Eq. 3.2 and 3.3. The logarithmic and exponential regression fit yielded $\mathrm{R}^{2}$ (the statistical correlation coefficient) values $>0.9$ for all data sets.

\begin{tabular}{|c|c|c|c|c|}
\hline \multicolumn{5}{|c|}{ Excitation (time $=0$ to $1 \mathrm{~s})$} \\
\hline$\lambda(\mathrm{nm})$ & $\begin{array}{c}\text { Power } \\
(\mathrm{mW})\end{array}$ & $\mathrm{A}$ & $\mathrm{B}$ & $\mathrm{R}^{2}$ \\
\hline \multirow{4}{*}{1110} & 890 & 0.350 & 1.767 & 0.95 \\
\cline { 2 - 5 } & 450 & 0.179 & 0.896 & 0.99 \\
\cline { 2 - 5 } & 300 & 0.117 & 0.585 & 0.96 \\
\cline { 2 - 5 } & 150 & 0.055 & 0.310 & 1.00 \\
\hline \multirow{4}{*}{1130} & 790 & 0.477 & 2.384 & 0.98 \\
\cline { 2 - 5 } & 450 & 0.233 & 1.137 & 0.97 \\
\cline { 2 - 5 } & 300 & 0.173 & 0.740 & 0.96 \\
\cline { 2 - 5 } & 150 & 0.073 & 0.359 & 1.00 \\
\hline \multirow{4}{*}{1150} & 735 & 0.517 & 2.783 & 0.97 \\
\cline { 2 - 5 } & 450 & 0.304 & 1.563 & 0.96 \\
\cline { 2 - 5 } & 300 & 0.173 & 0.963 & 0.95 \\
\cline { 2 - 5 } & 150 & 0.085 & 0.516 & 1.00 \\
\hline \multirow{4}{*}{1318} & 450 & 0.226 & 1.080 & 0.98 \\
\cline { 2 - 5 } & 300 & 0.136 & 0.691 & 0.93 \\
\cline { 2 - 5 } & 150 & 0.098 & 0.443 & 0.97 \\
\hline
\end{tabular}

\begin{tabular}{|c|c|c|c|c|}
\hline \multicolumn{5}{|c|}{ Relaxation (time $=1$ to $3 \mathrm{~s})$} \\
\hline \multirow{4}{*}{$\lambda(\mathrm{nm})$} & $\begin{array}{c}\text { Power } \\
(\mathrm{mW})\end{array}$ & $\mathrm{E}$ & $\mathrm{F}$ & $\mathrm{R}^{2}$ \\
\hline \multirow{4}{*}{1110} & 890 & 43.326 & -3.507 & 0.99 \\
\cline { 2 - 5 } & 450 & 59.378 & -4.400 & 0.99 \\
\cline { 2 - 5 } & 300 & 191.020 & -6.112 & 1.00 \\
\cline { 2 - 5 } & 150 & 137.820 & -6.660 & 1.00 \\
\hline \multirow{4}{*}{1130} & 790 & 43.381 & -3.358 & 0.99 \\
\cline { 2 - 5 } & 450 & 83.166 & -4.585 & 0.99 \\
\cline { 2 - 5 } & 300 & 411.060 & -6.467 & 0.98 \\
\cline { 2 - 5 } & 150 & 634008.694 & -8.916 & 1.00 \\
\hline \multirow{4}{*}{1150} & 735 & 48.090 & -3.106 & 0.98 \\
\cline { 2 - 5 } & 450 & 35.140 & -3.280 & 0.98 \\
\cline { 2 - 5 } & 300 & 45.433 & -3.944 & 0.99 \\
\cline { 2 - 5 } & 150 & 56.997 & -4.849 & 0.99 \\
\hline \multirow{3}{*}{1318} & 450 & 46.564 & -3.785 & 0.99 \\
\cline { 2 - 5 } & 300 & 21.540 & -3.519 & 1.00 \\
\cline { 2 - 5 } & 150 & 30.625 & -4.731 & 1.00 \\
\hline
\end{tabular}

The value for $\mathrm{R}$ at $\mathrm{t}=100$ and $250 \mathrm{~ms}$ was evaluated using Eq. 3.2 and the coefficients given in Table 3.3 to analyze $\mathrm{R}$ as a function of power, $\mathrm{P}$. These results are 
plotted in Fig. 3.11 and were fit to a linear regression line, with a zero-intercept, in Matlab.

(a)

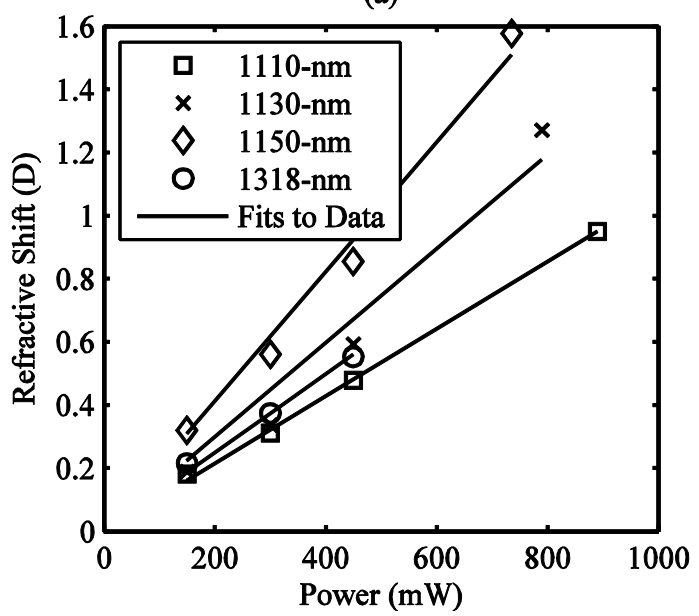

(b)

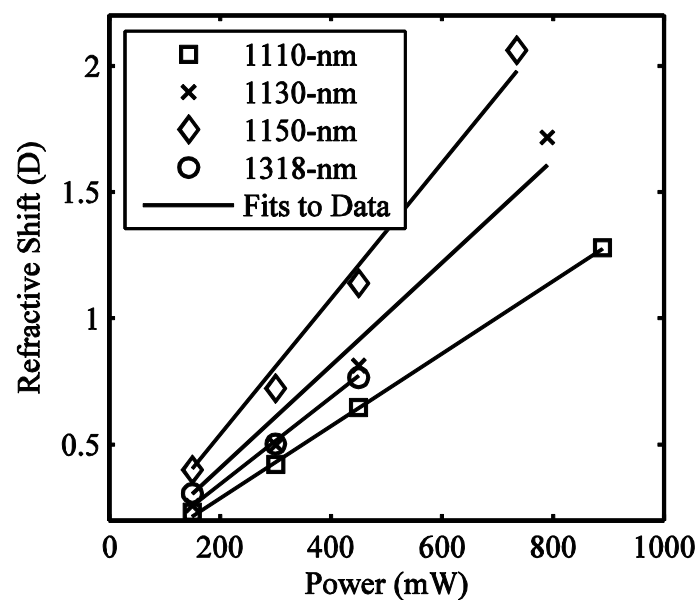

Figure 3.11: The relationship between refractive error and power for each wavelength evaluated at (a) 100-ms and (b) 250-ms exposures. The fits to data were generated by a linear fitting algorithm with a forced zero-intercept in Matlab.

The linear regression fits depicted in Fig. 3.11 follow the generic form of

$$
\mathrm{R}=\mathrm{mP} \text {, }
$$

where $R$ is the refractive shift $[D], m$ is the slope of the line $[\mathrm{D} / \mathrm{mW}]$ and $\mathrm{P}$ is power [W] delivered to the surface of the Cain-cell lens. The intercepts of the linear fits were forced to zero since there is no change in refractive error when no power is input into the system. Values for $\mathrm{m}$ are given in Table 3.4.

Table 3.4: The values for slope, $\mathrm{m}$, from the linear fits depicted in Fig. 3.11 fitting the generic form in Eq. 3.4 at time, $\mathrm{t}$, during the $\mathrm{CW}$ exposure.

\begin{tabular}{|c|c|c|c|c|}
\cline { 2 - 5 } \multicolumn{1}{c|}{} & \multicolumn{2}{c|}{$\mathrm{t}=100 \mathrm{~ms}$} & \multicolumn{2}{c|}{$\mathrm{t}=250 \mathrm{~ms}$} \\
\hline$\lambda(\mathrm{nm})$ & $\mathrm{m}(\mathrm{D} / \mathrm{mW})$ & $\mathrm{R}^{2}$ & $\mathrm{~m}(\mathrm{D} / \mathrm{mW})$ & $\mathrm{R}^{2}$ \\
\hline 1110 & 0.0011 & 1.00 & 0.0014 & 1.00 \\
\hline 1130 & 0.0015 & 0.96 & 0.0020 & 0.97 \\
\hline 1150 & 0.0021 & 0.99 & 0.0027 & 0.99 \\
\hline 1318 & 0.0012 & 0.98 & 0.0017 & 0.97 \\
\hline
\end{tabular}




\subsection{DISCUSSION}

At the end of 5-min of continuous exposure to $760-\mathrm{mW}$ of $1318-\mathrm{nm}$ laser radiation, the entire cuvette of vitreous was warm to the touch and measured up to $50.1^{\circ} \mathrm{C}$, but was determined to have no visible signs of whitening. Literature reports indicate temperatures above $54^{\circ} \mathrm{C}$ are needed to denature the proteins found in intraocular media (Swann, constable et al. 1972; Bailey, Sims et al. 1993). Based on literature reports and the observations made in this study, the vitreous did not denature from this exposure condition because the peak temperature remained sufficiently below $54^{\circ} \mathrm{C}$.

The density of the vitreous sample was determined to be $1.0149 \mathrm{~g} / \mathrm{mL}$, or approximately $5 \%$ greater than that of water. Vitreous is slightly more dense than water and contains some proteins in the matrix, but does not appear to denature very readily from near-infrared laser radiation. For the 1318-nm laser radiation wavelength the linear absorption coefficients of vitreous and water are 1.90 and $1.34 \mathrm{~cm}^{-1}$ respectively (Maher 1978).

Based on the results from Fig. 3.7, the thermal lens induced in the vitreous is nearly identical to that of a water sample for a CW 1313-nm laser radiation exposure. The beam-profiles displayed for the initial-state in Fig. 3.6a demonstrate the aberrations (spherical, coma, astigmatism) which are problematic with a short focal length geometry which is not optimally designed for mimicking the eye. However, the confocal imaging system allows for the tracking the approximate location of the beam-waist minimum without interference from optical aberrations. The excitation of the vitreous and water samples overlapped well within the error-bars of the experiment. The shape and size of the beam-diameter at each of the $\Delta \mathrm{z}$ imaging planes during the 1-s excitation was a near 
match between vitreous and water at 129-mW of 1313-nm laser radiation (Fig. 3.6 a \& b). The size and shape of the beam-diameters at each of the $\Delta \mathrm{z}$ imaging planes during the thermal relaxation for the vitreous and water also closely match. Based on observational analysis of the thermal relaxation data (Fig. $3.6 \mathrm{c} \& \mathrm{~d}$ ) of the vitreous compared to water, the vitreous appears to take slightly more time to dissipate the absorbed energy when relaxing back to initial-state, however, the difference falls within the uncertainties of the experimental design (see error bars in Fig. 3.7).

Given the observations that vitreous remains sufficiently below the denaturing temperature of $54^{\circ} \mathrm{C}$ even with high linear absorption for CW NIR laser radiation and the fact that the thermal lens effect in water and vitreous appears experimentally equivalent under the same conditions (Fig. 3.6), water is a suitable substitute for investigating the thermal lens. From this conclusion, I find the use of water in the Cain-cell artificial eye, which is designed to minimize initial-state optical aberrations, to be an excellent experimental tool for studying the thermal lens. This tool assists with reducing the need for animal experimentation and cost associated with studying the thermal lens in the eye.

The beam-profiles shown at initial-state in Fig. 3.9a appear to have an improvement in aberrations when using the Cain cell compared to the initial-state beam profiles seen in Fig. 3.6a which used a 25.4- $\mathrm{mm}$ lens and quartz cuvette as the artificial eye. Using the confocal imaging technique set at a series of $z$-space positions allows for information about the thermal lens through both time and space regardless of aberrations in the artificial eye. Using the Cain cell, data (Fig. 3.9) can be processed in terms of the $\Delta \mathrm{z}$ relative to time yielding information on the strength of the thermal lens in terms of a refractive shift, R, in Diopters, D (Fig. 3.10). The transient response of $\mathrm{R}$ is shown to be logarithmic in nature, reaching steady-state in a few $100 \mathrm{~ms}$. The data in Fig. 3.9 show 
that the $1150-\mathrm{nm}$ wavelength exhibits a stronger thermal lens over the other wavelengths as evident by the amount of z-shift or defocus for each of the wavelengths. Though the linear absorption coefficient of water is largest for the 1318-nm followed by 1150,1130 and finally $1110-\mathrm{nm}$, the strength of the thermal lens does not follow this trend.

The strength of the thermal lens depends on the generation of heat related to two key elements; 1 - the linear absorption coefficient and 2 - the energy density of the laserbeam's profile created at the focus. As a result, when the same input power is exposed to the artificial eye, the $1150-\mathrm{nm}$ wavelength $\left(\mu_{\mathrm{a}}=0.78 \mathrm{~cm}^{-1}\right.$ and $\left.\% \mathrm{~T}=22.5 \%\right)$ exhibits a stronger thermal lens, as quantified by refractive shift, compared to the 1318-nm wavelength $\left(\mu_{\mathrm{a}}=1.34 \mathrm{~cm}^{-1}\right.$ and $\left.\% \mathrm{~T}=3 \%\right)$. These two key elements are what drive the temperature rise in the sample media. Thus under identical input conditions, the 1150$\mathrm{nm}$ wavelength will result in more heat generated at the focal plane, and subsequently a stronger thermal lens, compared to the 1318-nm wavelength. Furthermore, the linear relationship depicted in Fig. 3.11 between $\mathrm{R}$ and power, $\mathrm{P}$, indicate that the thermal lens follows a first-order response. The trend in the slopes between wavelengths (Table 3.4) describes that thermal lensing from 1150-nm laser radiation is the greatest, followed by 1130,1318 and finally $1110 \mathrm{~nm}$. This trend is due to power density delivered in the Rayleigh range of the optical system (the Cain cell) and the linear absorption coefficient, $\mu_{\mathrm{a}}$, of the absorbing media (water), suggesting that under the same input conditions, the temperature rise is greatest for 1150-nm followed by 1130, 1318 and 1110-nm wavelengths. Mathematical models detailing this relationship for an optical configuration similar to the eye are detailed in Chapter 4.

Information on the distribution of the light intensity for a $\Delta \mathrm{z}$ position is also revealed using this technique, showing how the probe laser-beam's profile begins to form 
a donut in spatial positions for which the minimum-beam waist has moved through (Fig. 3.9), i.e. - notice the donut in Fig. $3.9 \mathrm{~b}$ for the $1150-\mathrm{nm}$ wavelength at spatial planes for $\Delta \mathrm{z}=-50,0$, and $50 \mu \mathrm{m}$. The pinhole-free confocal imaging technique overcomes the inherent difficulties produced by the formation of the donut and has proven to be an excellent analytical tool for understanding the thermal lens in an eye-like geometry.

\subsection{CONCLUSION}

A confocal imaging technique provides valuable insight into the formation of the thermal lens in an artificial eye including data for the transient response of spatial information in $\mathrm{x}, \mathrm{y}$ and $\mathrm{z}$ space. The thermal lens of vitreous and water appear to be the same, making the use of a low-cost water-filled Cain cell artificial eye an optimal choice for investigating the thermal lens. Though thermal lensing appears strongest for the 1150-nm wavelength in Fig. 3.9, Fig. 3.10, and Fig. 3.11, the linear absorption coefficient at 1318-nm is much larger. However, attenuation causes the heat generation from 1318$\mathrm{nm}$ to be less than the $1150-\mathrm{nm}$ when input conditions are equivalent. These data show thermal lensing occurs upon exposure to NIR laser radiation logarithmically in time, but linearly with power.

\subsection{ACKNOWLEDGEMENTS}

The author would like to thank Dr. Jeff Oliver from the Air Force Research Laboratory and Mr. Gary Noojin for their assistance with data collection for this chapter and the USAF School of Aerospace Medicine for their assistance with obtaining tissue samples. 


\section{CHAPTER 4}

\section{A FIRST-ORDER MODEL OF THERMAL LENSING IN A VIRTUAL EYE}

\subsection{ABSTRACT}

An ABCD beam-propagation method was used to build a first-order mathematical model of a thermal lens effect from a near-infrared laser beam in water and ocular media. The model was found to fit experimental z-scan data best when the thermo-optic

coefficient, $\mathrm{dn} / \mathrm{dT}$, of liquid water at $292 \mathrm{~K}$ was $-4.46^{*} 10^{-5} \mathrm{~K}^{-1}$. The physiological parameters of the human eye were simulated in a simple eye model using this fitted $\mathrm{dn} / \mathrm{dT}$ value. Conservative model simulations for 1150 and 1318-nm laser radiation include parameter sets used in experimental ocular exposures performed by Zuclich, Lund et al. 2007, to illustrate the transient response of the thermal lens approaching the limits of the retinal damage thresholds for equivalent laser radiation sources. The content in this chapter has been published in Vincelette, Thomas et al., Journal of the Optical Society of America A, 2009.

\subsection{INTRODUCTION}

A review of the retinal damage studies from 1980-2004 was detailed in Chapter 2 to explain how thermal lensing may have contributed to the difficulties encountered by researchers when trying to establish retinal damage thresholds from continuous-wave, $\mathrm{CW}$, near-infrared, NIR, laser radiation. I have hypothesized that thermal lensing plays a significant role in laser-tissue interactions influencing ocular damage for wavelengths 
from $1150-1350 \mathrm{~nm}$, where pre-retinal media strongly absorb laser radiation yet there is enough energy remaining to pose a threat to the sensitive retinal layers. In Chapter 3 , the thermal lens in a water-filled eye exposed to CW NIR laser radiation demonstrated that thermal lensing at $1150-\mathrm{nm}$ is stronger than the $1318-\mathrm{nm}$ wavelength. The strength of the thermal lens was determined to be related to the linear absorption of the exposed sample and the energy density formed in the medium. To best understand the laser-tissue interaction of thermal lensing in ocular media, a mathematical model describing the phenomena in a virtual eye was developed and tested against a simple thermal lens experiment for validation.

Thermal retinal damage models use laser induced heat generation, heat conduction and the Arrhenius damage integral to predict the formation of a laser induced lesion in the retina (Clark, Irvin et al. 2008). These models require an understanding of laser beam spot size and energy at the retina which depend upon optical properties of the pre-retinal media including dispersion, absorption, and physical dimensions of the eye. Similar fundamental understandings are necessary to estimate an action spectra used to make predictions on general trends by fitting available MVL data over the entire UVvisible-IR spectrum (Lund and Edsall 1999; Lund, Edsall et al. 2000; Zuclich, Lund et al. 2007; Vincelette, Rockwell et al. 2008). To date, thermal lensing has been a relatively unexplored laser-tissue interaction in the eye and has not been incorporated in any eye model used to predict retinal damage thresholds.

The discovery of thermal lensing by Gordon, Leite et al. in 1965 led to the development of numerous mathematical models. The phenomenon is the result of a temperature gradient, typically assumed to be radially symmetric, formed by the absorption of laser light in the medium. As the temperature, $\mathrm{T}$, of the medium increases, 
the local density, $\rho$, decreases. This leads to a decrease in the index of refraction, $n$, resulting in the formation of a negative lens. The temperature gradient is shaped by the beam profile and the thermal diffusivity of the media (Fang and Swofford 1979; St. John, Taheri et al. 1992; Kovsh, Yang et al. 1999; Kovsh, Hagan et al. 1999). The creation of a thermal lens in ocular media means the spot size formed at the retina changes dynamically as a function of the coupled transient response of heat generated by the beam profile and thermal diffusion.

Many thermal lensing models begin with a solution of the heat conduction equation in combination with an ABCD ray-tracing method (Fang and Swofford 1979; Yariv 1989; St. John, Taheri et al. 1992). The model assumes a radial symmetric temperature gradient in an absorbing medium where no heat is exchanged along the optical axis, z.

A closed aperture z-scan is one technique to correlate transmission data through an absorbing medium to a thermal lens gradient (Franko and Tran 1996). A single-beam closed-aperture z-scan using 1313-nm light provides an estimate of thermal lensing in water. The thermal characteristics of water are well documented and have similar linear absorption coefficients to ocular media at NIR wavelengths making the z-scan a basic method for initial evaluation of the thermal lensing model (Hale and Querry 1973; Maher 1978; Schiebener, Straub et al. 1990; Harvey, Gallagher et al. 1998; Wagner and Kruse 1998).

In this chapter, data from a single-beam closed-aperture z-scan using a continuous wave $(\mathrm{CW})$ 1313-nm laser radiation source are presented and compared to a first-order model for thermal lensing. Data from the z-scan are used as a means to determine an estimate for the value of the change in index of refraction with the change in temperature, 
$\mathrm{d} n / \mathrm{d} T$, for this region of laser radiation. Having demonstrated a reasonable fit to $\mathrm{z}$-scan data, the first-order model is then used as a tool for predicting trends of the thermal lensing effect in a virtual eye. Preliminary data relating to this work have been reported (Thomas, Vincelette et al. 2005; Vincelette, Thomas et al. 2006; Thomas, Vincelette et al. 2007; Vincelette, Thomas et al. 2007). The first-order model presented here does not include heat conduction along the z-axis nor the effects of convection in the absorbing media. The more viscous properties of ocular media compared to water mitigates significant convectional flow.

\subsection{BACKGROUND}

\subsubsection{Z-Scan Spectroscopy}

The Z-scan is a common analytical technique used to measure the thermal lens effect. The technique is a well established method in chemistry for chromatography and electrophoresis analyses and in electro-optics for testing the characteristics of optical limiters (Fang and Swofford 1979; Sheik-Bahae, Said et al. 1990; St. John, Taheri et al. 1992; Kozich, Hernandez et al. 1995; Franko and Tran 1996; Kovsh, Yang et al. 1999; Kovsh, Hagan et al. 1999).

A single-beam z-scan is the simplest of z-scan techniques, where a single laser is used as the pump and probe. The laser beam is collimated and aligned to be sent through a focusing lens. A chopper or shutter placed after the focusing lens is traditionally used as the control for the exposure duration (Franko and Tran 1996). The sample is placed at position, $z$, in the beam's path, after the shutter, at an angle just a few degrees off from the z-axis to prevent reflective interference from the cuvette windows. A detector is placed in the far-field from the focusing lens. An aperture is placed just in front of the 
detector, giving the nomenclature of a "closed-aperture z-scan." Irradiance data are collected as a function of time as the sample is held in a stationary position. The sample is allowed time to relax back to its initial thermal state, then moved to a new z-position before beginning the next exposure. Conventional z-scan data report the z-position of the sample in relation to the distance from the focal plane of the focusing lens. The absorption of laser radiation in liquid media causes the formation of a negative thermal lens. The irradiance measurements are analyzed at a selected time and normalized to a reference irradiance signal taken for the sample placed at a far distance, approximately one focal length, from the focus of the lens (Fig. 4.1). The z-scan data (Fig. 4.1d) depict an increase (Fig. 4.1b) and decrease (Fig. 4.1c) in relative irradiance for sample positions before and after the focus respectively, with an inflection point occurring at the focal plane of the lens (Fig. 4.1a).

Selections of the lens and sample thickness are important for the z-scan. The irradiance of the thermal lens is largely related to the generation of heat $[\mathrm{W} / \mathrm{m} 3]$ in the sample, making the choice of a short focal length lens seem desirable. However, since the thermal lens measurement is very sensitive to confocal distance, Franko and Tran 1996, describe using a sample thickness, $\mathrm{T}_{\mathrm{S}}$, which is at least one-third or less than the confocal distance expressed as

$$
T_{S} \leq \frac{1}{3} \frac{2 \pi \omega_{\min }^{2}}{\lambda}
$$

Where $\omega_{\min }$ is the minimum $1 / \mathrm{e}^{2}$ beam waist radius of the probe beam with wavelength, $\lambda$ (Franko and Tran 1996). The minimum waist can be approximated as $f \lambda / 2 d$ where $d$ is the beam's $1 / \mathrm{e}^{2}$ diameter at the focusing lens with focal length, $f$. As a result, the selected sample path length is proportional to the confocal distance, or twice the Rayleigh range, of the system design. 


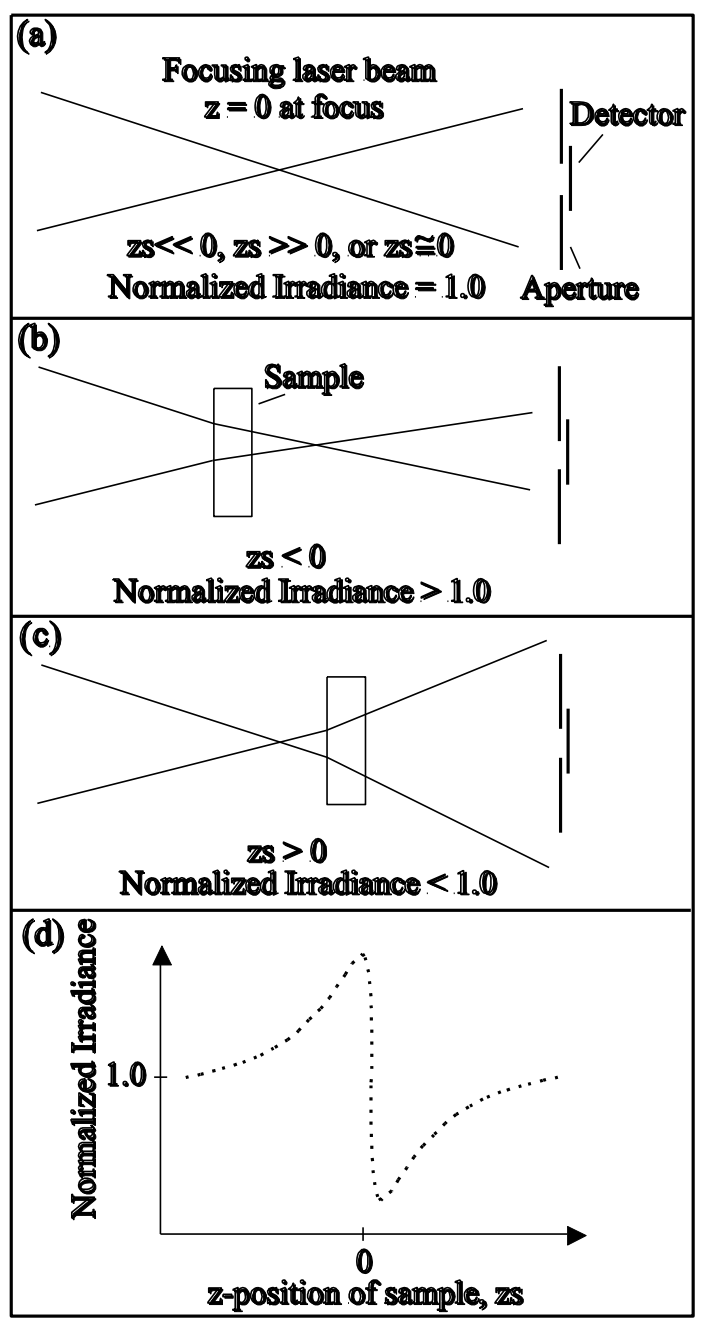

Figure 4.1: An example of a negative thermal lens z-scan. The case for the sample exposed at z-positions (a) very far from the focus of the lens and very near or at the focus resulting in unity normalized irradiance, (b) before the focus resulting in an increase in normalized signal irradiance, and (c) after the focus resulting in a decrease in signal irradiance. (d) The resulting z-scan data set.

A single-beam closed-aperture z-scan allows for only the transient information of the excitation of the sample (Franko and Tran 1996). When both the excitation and relaxation of the sample are desired, a dual-beam z-scan should be used. 


\subsubsection{Mathematical Model}

Using Yariv's approach for deriving the relationship of a media with a quadratic index of refraction to a ray, the index of refraction, $n$, can be described as a function of radial distance, $r$, from the optical axis, $z$, and temperature, $T$ (Yariv 1989). Using a Maclaurin series to expand an expression for $n(r, T)$ gives

$$
n(r, T)=n_{o}+r\left(\frac{\partial n}{\partial r}\right)_{r=0}+\frac{r^{2}}{2 !}\left(\frac{\partial^{2} n}{\partial r^{2}}\right)_{r=0} \ldots .
$$

Truncating the series and applying the chain rule gives

$$
n(r, T)=n_{0}+r\left(\frac{\partial n}{\partial T} \frac{\partial T}{\partial r}\right)_{r=0}+\frac{r^{2}}{2} \frac{\partial}{\partial r}\left(\frac{\partial n}{\partial T} \frac{\partial T}{\partial r}\right)_{r=0}
$$

The change in temperature with respect to radial distance at radial position $r=0$ can be set to 0 simplifying the expression to just the background index of refraction, $n_{o}$, and the last term containing the square of the radius, $r$. From this point, applying the product rule gives Eq. 4.4 which is then evaluated for $\partial T / \partial r \rightarrow 0$ at $r=0$, where the maximum irradiance of the beam is found, revealing the expression

$$
n(r, T)=n_{0}+\frac{r^{2}}{2}\left[\frac{\partial}{\partial r}\left(\frac{\partial n}{\partial T}\right)\right] \frac{\partial T}{\partial r}_{r=0}+\frac{r^{2}}{2}\left[\frac{\partial}{\partial r}\left(\frac{\partial T}{\partial r}\right)\right] \frac{\partial n}{\partial T}_{r=0}
$$

further reducing to

$$
n(r, T)=n_{0}+\frac{r^{2}}{2} \frac{\partial^{2} T}{\partial r^{2}} \frac{\partial n}{\partial T_{r=0}}
$$

The background index of refraction, $n_{0}$, must be selected for the corresponding media and wavelength of interest. The term $\partial^{2} T / \partial r^{2}$ is solved using a Green's function solution to the heat conduction equation explained shortly (Fang and Swofford 1979; St. John, Taheri et al. 1992; Kovsh, Hagan et al. 1999). For cases where the laser pulse is longer than the acoustic transit time, as is the case with $\mathrm{CW}$ laser exposures, the change 
in $n, \Delta n$, can be approximated by $d n / d T^{*} \Delta T$ (Kovsh, Yang et al. 1999). The term $d n / d T$ is referred to as the thermo-optic coefficient and is wavelength dependent (Lin 1997; Kovsh, Hagan et al. 1999).

An equivalent expression for $n(r, T)$ for a medium with a quadratic index of refraction is described by Yariv as (Yariv 1989)

$$
n=n_{0}\left(1-\frac{X r^{2}}{2}\right) .
$$

A solution for $X$ is determined by considering only $\mathrm{CW}$ cases and setting Yariv's equation for $n$, Eq. 3.6, equal to the solution for $n(r, T)$, Eq. 4.5, giving

$$
X=\frac{1}{n_{0}} \frac{d n}{d T} \frac{\partial^{2} T}{\partial r^{2}} .
$$

The term $X$ is needed to describe how a ray would move through a quadratic index medium using an $\mathrm{ABCD}$ propagation matrix. The $\mathrm{ABCD}$ matrix for propagating a beam through a negative-lens-like medium is given as (Yariv 1989)

$$
\left[\begin{array}{ll}
A & B \\
C & D
\end{array}\right]=\left[\begin{array}{cc}
\cosh \left(X^{\frac{1}{2}} \Delta z\right) & X^{\frac{1}{2}} \sinh \left(X^{\frac{1}{2}} \Delta z\right) \\
X^{\frac{1}{2}} \sinh \left(X^{\frac{1}{2}} \Delta z\right) & \cosh \left(X^{\frac{1}{2}} \Delta z\right)
\end{array}\right] .
$$

The term $\Delta z$ is the axial step or length of the step through which the beam is propagating. A complex beam parameter, $q(z)$, is used to describe the ray at position, $z$, anywhere along the $\mathrm{z}$-axis in a medium in as

$$
q(z)_{\text {new }}=\frac{A q(z)_{\text {old }}+B}{C q(z)_{\text {old }}+D} .
$$

The complex beam parameter is described by the real and imaginary portions of a wavefront by 


$$
\frac{1}{q(z)}=\frac{1}{R(z)}-i \frac{m^{2} \lambda}{\pi \omega(z)^{2}},
$$

where the real portion of the complex beam parameter contains information about the radius of curvature $R(z)$, while the imaginary portion contains the wavelength traversing the medium and the $1 / \mathrm{e}^{2}$ beam radius, $\omega(z)$. The value of $m^{2}$, or $m$-squared, describes the quality of collimation of the beam (Belanger 1991; Porras, Alda et al. 1993). Ideally, a Gaussian beam would have an $m^{2}$ value of 1.0, but realistically, the value is greater than 1.0 .

To solve for the $\partial^{2} T / \partial r^{2}$, the eye can be treated as a cylindrically symmetric medium with thickness $z$. For our first-order model, thermal diffusion along the optical axis is assumed to be zero $(\partial T / \partial z=0)$. Thus, we assume diffusion occurs along the radial direction. However, the heat-source term, $S(z)$, is attenuated in the z-direction according to Beer's Law, Eq. 4.15. The thermal diffusion equation,

$$
\frac{1}{r} \frac{\partial}{\partial r}\left[\kappa r \frac{\partial T}{\partial r}\right]+S(z)=\rho c_{p} \frac{\partial T}{\partial t},
$$

must be solved first by differentiating the heat equation with respect to $(\boldsymbol{r}, t)$, then integrated to find $\partial^{2} T / \partial r^{2}$. Where $t$ is time, $r$ is the magnitude of the radial vector described by position (x,y), $\eta$ is the thermal diffusivity in $\mathrm{cm}^{2} / \mathrm{s}$, and $S(z)$ is the source term. Note: $\eta=\kappa / \rho c_{p}$ where $\kappa$ is the thermal conductivity in $\mathrm{W} /(\mathrm{cm} \mathrm{K}), \rho$ is the density in $\mathrm{g} / \mathrm{cm}^{3}$ and $c_{p}$ is the specific heat at constant pressure in $\mathrm{J} /(\mathrm{Kim}$ and $\mathrm{Su})$. The eye was modeled as an infinite cylinder with heat generation originating from the Gaussian beam launched into the eye. Since the conduction of heat is assumed to be purely a radial process, the diffusion equation is independent of angle and axial position allowing the 
radial vector, $\boldsymbol{r}$, to reduce to a scalar radial magnitude, $r$ (Wyld 1976; Beck, Cole et al. 1992).

Applying the product rule and rearranging variables in Eq. 4.11 gives the heat equation

$$
\frac{\partial^{2} T}{\partial r^{2}}+\frac{1}{r} \frac{\partial T}{\partial r}+\frac{1}{\kappa} S(z)=\frac{1}{\eta} \frac{\partial T}{\partial t} .
$$

The Green's function for a virtual cylindrical eye with no diffusion along $z$ is taken as

$$
G\left(r, t ; r^{\prime}, t^{\prime}\right)=-\frac{1}{4 \pi \eta\left(t-t^{\prime}\right)} \exp \left[-\frac{\left(r^{2}+r^{\prime 2}\right)}{4 \eta\left(t-t^{\prime}\right)}\right] I_{0}\left[\frac{r r^{\prime}}{2 \eta\left(t-t^{\prime}\right)}\right],
$$

where $r^{\prime}$ describes the position of the cylindrical surface heat source and $I_{0}$ is the modified Bessel function of the first kind (Beck, Cole et al. 1992; St. John, Taheri et al. 1992). The units of this Green's function are in $\mathrm{cm}^{-2}$. $G\left(r, t ; r^{\prime}, t^{\prime}\right)=0$ for all positions where $r$ is on the surface of the cylindrical medium. Since only continuous wave (CW) cases are being considered here, $t^{\prime}=0$.

Applying a Green's function, Eq. 4.13, to the heat diffusion equation, Eq. 4.12, for a symmetrical Gaussian beam results in

$$
T(z, r, t)=\frac{1}{\rho c_{p}} \int_{0}^{t} d t^{\prime} \int_{0}^{2 \pi} d \theta \int_{0}^{\infty} r^{\prime} d r^{\prime} S\left(z, r^{\prime}\right) G\left(r, t ; r^{\prime}\right) .
$$

The expression

$$
S\left(z, r^{\prime}\right)=\frac{2 \mu_{a} P_{z}}{\pi \omega^{2}} \exp \left[\frac{-2\left(r^{\prime}\right)^{2}}{\omega^{2}}\right],
$$

describes the source term, $S\left(z, r^{\prime}\right)$, for the symmetrical Gaussian beam, for the eye in $\mathrm{W} / \mathrm{cm}^{3}$ can be found by applying Beer's law assuming a Gaussian beam (Fang and Swofford 1979; St. John, Taheri et al. 1992). In Eq. 4.15, $\mu_{a}$ is the linear absorption 
coefficient in $\mathrm{cm}^{-1}, \omega$ is the $1 / \mathrm{e}^{2}$ beam radius, and $r^{\prime}$ denotes the location of the heat source. $P_{z}$ is the power in Watts delivered at position, $z$, in the sample given by Beer's law as $P_{z}=P_{0} * \exp \left(-\mu_{a} z\right)$, with $P_{0}$ as the initial power delivered at the front surface of the sample. The power is made into an array of values by Beer's law through $P_{z+\angle z}=$ $P_{0} * \exp \left(-\mu_{a} z\right) * \exp \left(-\mu_{a} * \Delta z\right)$, where $z$ is the position along the z-axis within the sample and $\Delta z$ is the same spatial step described earlier in Eq. 4.8.

Using Mathematica ${ }_{\circledast} 5.2$, a solution for $\partial^{2} T / \partial r^{2}$ was verified to be

$$
\frac{\partial^{2} T}{\partial r^{2}}=\frac{-\eta 8 \mu_{a} P_{z}}{\pi \kappa \omega^{2}}\left[\frac{t}{8 \eta t+\omega^{2}}\right] .
$$

The beam and subsequent radial temperature profiles are assumed to be Gaussian over all time, t. The critical time is defined by $t_{c}=\omega^{2} / 8 \eta$, allowing the solution to reduce to

$$
\frac{\partial^{2} T}{\partial r^{2}}=\frac{-\mu_{a} P_{z}}{\pi \omega^{2} \kappa}\left[\frac{1}{1+t_{c} / t}\right] .
$$

Analysis of the problem can include observing the temperature rise along the optical axis. Given our assumption of $\partial T / \partial z=0$ and initial condition $\partial T / \partial t=0$, the temperature rise along the $\mathrm{z}$-axis is representative of the central temperature resulting from diffusion along the radius, $r$. The temperature rise along the z-axis at $r=0$ is then determined by evaluating the heat conduction equation at $r=0$, giving (this requires applying L'Hopital's Rule to the term $\left.(1 / r)^{*} \mathrm{~d} T / \mathrm{d} r\right)$

$$
\left.\left(\frac{\partial T}{\partial t}\right)\right|_{r=0}=\left.\eta\left(2 \frac{\partial^{2} T}{\partial r^{2}}+\frac{1}{\kappa} S(z)\right)\right|_{r=0} .
$$

The source term at $r=0$ simplifies to $S(z)=2 \mu_{a} P_{z} / \pi \omega^{2}$, where $P_{z}$ was previously described. 
Numerical integration, of Eq. 4.18, with respect to time can then be used to determine an approximate solution to the temperature, $\mathrm{T}$. A uniform temperature, $T_{0}$, is assumed when $t_{0}=0$ with an initial beam radius, $\omega_{0}$, created from the optical geometry of the system with no thermal lensing. Methods for solving this integral have been reported in Fang and Swofford 1979. Analysis of the temperature rise is reserved for Chapter 6.

\subsection{MATERIALS AND METHODS}

\subsubsection{Single-Beam, Closed-Aperture Z-Scan}

A 1313-nm IRCL-150-1313-P-L CrystaLaser was collimated and aligned (Fig. 4.2) to create a $1 / \mathrm{e}^{2}$ beam diameter at the focusing lens of $3.5 \mathrm{~mm}$ and $\mathrm{an}^{2}$ of 1.16 . A 400-mm BK7 planoconvex lens was used as the focusing lens. A knife-edge test found the 1313-nm laser focus to be $42 \mathrm{~cm}$ from the center of the lens.

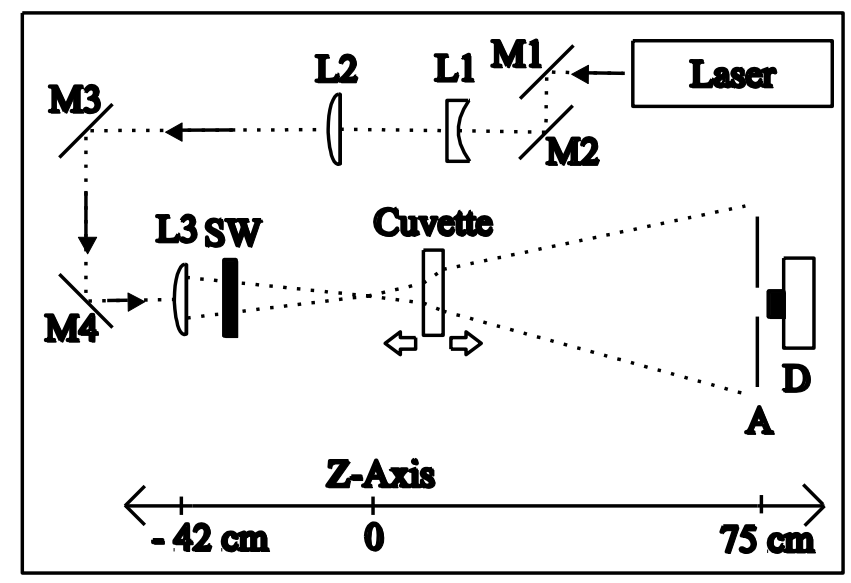

Figure 4.2: The closed-aperture, single-beam z-scan set up. The z-axis is referenced to the focal position of the lens found using a knife-edge technique in air, with no cuvette in the beam's path. The laser was a 1313-nm CrystaLaser IRCL-150-1313-P-L. M1-M4: IR-coated mirrors. L1 and L2: IR-coated lenses created a beam expander of $\sim 1.5 \mathrm{x}$. L3: 400-mm ThorLabs plano-convex 1-in lens. SW: 1-cm diameter Uniblitz shutter. Quartz Cuvette: from Starna Cells. A: Aperture set to 2.5-mm diameter. D: 818-IR Newport detector. 
The power of the 1313-nm laser was attenuated by using a Thorlab NEK01 neutral density (ND) filter set. A Uniblitz 10-mm shutter was used to control the delivery of the laser beam to the sample. The focusing lens and any ND filters were placed before the Uniblitz shutter to allow for any thermal lens in the optical components to remain in steady-state throughout the duration of the experiment.

All experiments were run at room temperatures between $17-20{ }^{\circ} \mathrm{C}$. A sample of ultra-pure deionized water was placed in a 10-mm path-length quartz cuvette purchased from Starna Cells Inc. Samples were run in sets at 100 and 1000-ms exposures for powers of 2.60 and $48 \mathrm{~mW}$. The linear absorption coefficient of water for 1313-nm was estimated to be $1.27 \mathrm{~cm}^{-1}$ (Hale and Querry 1973).

A Newport 818-IR detector was placed $117 \mathrm{~cm}$ down the optical path from the focusing lens. A 2.5-mm aperture placed in front of the detector was found to yield the largest dynamic range in normalized irradiance data for our z-scan system. The irradiance signal from the detector was sent to a Melles Griot large gain transimpedance amplifier before being recorded in Labview. A Stanford Research Systems signal delay generator was used to synchronize the data acquisition with the shutter. The sample was then placed on a stage along the optical path of the focusing lens ranging from -18 to 22 $\mathrm{cm}$ in 1-cm increments relative to the focal position of the lens. The irradiance values were normalized by irradiance values measured for a sample position at least $22 \mathrm{~cm}$ from the focus.

In earlier reports of the first-order thermal lensing model, I used constant thermal coefficients and discovered a strong sensitivity in the early model to small changes in these coefficients (Vincelette, Thomas et al. 2006). To model the z-scan experiments presented here, values for thermal conductivity, $\kappa$, specific heat at constant pressure, $c_{p}$, 
and density, $\rho$, as a function of temperature, $T$, were obtained from Wagner and Kruse 1998, at $0.1 \mathrm{MPa}$. Data between $10-90{ }^{\circ} \mathrm{C}$ were fit to a polynomial in Excel to obtain continuous temperature-dependent thermal properties. These polynomials are given in Table 4.1. Thermal diffusivity, $\eta$, was calculated from $\eta=\kappa / \rho c_{p}$, then fit to a polynomial. The linear regression $\mathrm{R}^{2}$ value was greater than 0.995 for all polynomial fits to the Wagner and Kruse 1998 data.

Table 4.1: Thermal conductivity, $\kappa$, density, $\rho$, specific heat at constant pressure, $\mathrm{c}_{\mathrm{p}}$, and thermal diffusivity, $\eta$, as a function of temperature, $T$, found to fit data for liquid water from Wagner et al. for 0.1 MPa between 283.15 to 363.15 K (Wagner and Kruse 1998).

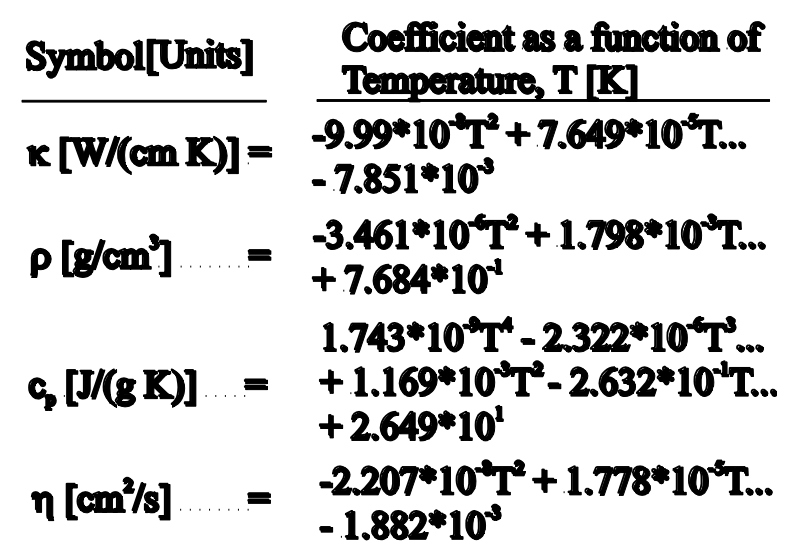

\subsubsection{Model of the Virtual Eye}

The eye is a complex optical system, but for our purposes in assessing trends of thermal lensing in an eye, we have simplified our model eye down to a basic compartmental system consisting of a single curved surface. Thermal lensing in a simple eye model was simulated for two wavelengths: 1318-nm; as this was the wavelength used in retinal $\mathrm{ED}_{50}$ studies by Zuclich, Lund et al. 2004, and 1150-nm; to contrast the variation on thermal lensing from a change in absorption coefficients. The eye was modeled as a four-chamber compartment broken into cornea, aqueous, lens and vitreous chambers with a radius of curvature existing only on the front surface of the air-cornea 
boundary. This radius of curvature is essential in the $\mathrm{ABCD}$ beam propagation for our thermal lensing model. Linear absorption coefficients for each of these ocular media for 1150 and $1318 \mathrm{~nm}$ are in Table 4.2. A review of literature found the average thickness of these compartments for a human to be $0.05,0.31,0.36$ and $1.72 \mathrm{~cm}$ for cornea, aqueous, lens and vitreous respectively (Fig. 2.4) (Li, Zwick et al. 2000; Atchison and Smith 2002). With a known refraction and physiological distance to a virtual retinal plane of $2.44 \mathrm{~cm}$, a radius of curvature, $\mathrm{r}_{\mathrm{c}}$, of $0.61 \mathrm{~cm}$ was found to set the $589-\mathrm{nm}$ wavelength to a minimum focus at $z=2.44 \mathrm{~cm}$, completing the model eye geometry shown in Fig. 4.3 .

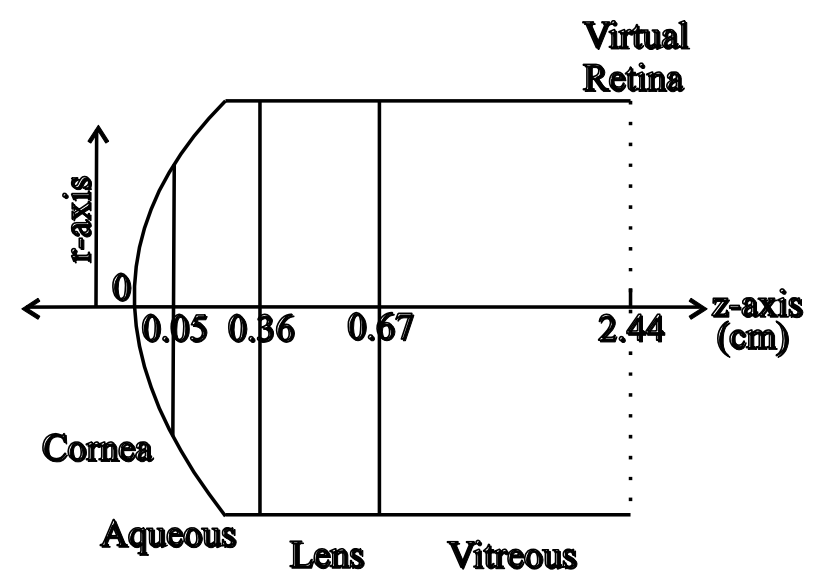

Figure 4.3: The simple eye model with a radius of curvature of $0.61 \mathrm{~cm}$ on the front surface of the cornea brings a collimated, 589-nm beam to a minimum at the virtual retinal plane $2.44 \mathrm{~cm}$ after the front surface of the eye. The vitreous layer is infinitely long, with beam trend observations made at the position of the virtual retina.

Table 4.2: Linear absorption coefficients for ocular media and water at 1150 and 1318 nm (Hale and Querry 1973; Maher 1978).

\begin{tabular}{|c|ccccc|}
\hline$\lambda$ & \multicolumn{4}{|c|}{ Absorption Coefficient, $\mu_{a}\left[\mathrm{~cm}^{-1}\right]$} \\
\cline { 2 - 6 }$[\mathrm{nm}]$ & Cornea & Aqueous & Lens & Vitreous & Water \\
\hline 1150 & 1.51 & 1.22 & 0.61 & 1.13 & 1.78 \\
1318 & 1.98 & 2.01 & 0.97 & 1.90 & 1.34 \\
\hline
\end{tabular}


Refractive error measurements for wavelengths beyond 900-nm in the eye are limited, thus chromatic dispersion for wavelengths greater than 900 -nm were based upon data trends in Fernandez, Unterhuber et al. 2005. Using these trends in refractive error, a bulk refractive index value as a function of wavelength was determined by a Sellmeier fit, as described in Chapter 2 and in an earlier publication by Vincelette, Rockwell et al., 2008, to account for chromatic dispersion in the eye model. For a given wavelength, the initial values of $\omega(\mathrm{z})$ in the virtual eye are calculated from the $\mathrm{ABCD}$ propagation method described by Yariv from Eq. 4.6-10 using the corresponding bulk refractive index from the Sellmeier fit reported in Chapter 2 (see Table 2.5) (Vincelette, Rockwell et al. 2008). The thermal conductivity, $\kappa$, specific heat at constant pressure, $c_{p}$, and density, $\rho$, of ocular media were held as constants reported in literature (Okuno, Kojima et al. 2005).

\subsubsection{First-Order Thermal Lensing Mathematical Model}

The mathematical model presented earlier was built in Matlab $_{\circledast}$ to predict the thermal-lensing effect in a single-beam, closed-aperture z-scan and in the reduced eye model (see Appendix A for documentation of code for z-scan simulations). The thermallensing model requires an initial seed of $1 / \mathrm{e}^{2}$ beam-waist values based upon no thermal lensing; these become the beam-waist values at $t=0 \mathrm{~s}$. Subsequent $1 / \mathrm{e}^{2}$ waist values for $\mathrm{t}$ $>0$ are then found through an iterative process using small time steps initially, gradually increasing the time step size as the thermal lens reached steady-state.

A value for $\mathrm{dn} / \mathrm{dT}$ was selected for the model based on a best fit to the $\mathrm{z}$-scan data. This value of $\mathrm{dn} / \mathrm{dT}$ was then used in modeling the thermal lensing effect in the simple eye model.

To determine the thermo-optic coefficient from literature, the index of refraction data at $0.1 \mathrm{MPa}(\sim 1 \mathrm{~atm})$ for laser radiation at 404, 589, 632, 1013 and $2325 \mathrm{~nm}$ in water 
in the liquid phase between 10 and $90{ }^{\circ} \mathrm{C}$ as reported in literature were fit to third-order polynomials in Excel to determine $\mathrm{n}(\mathrm{T})_{\lambda}$ (Schiebener, Straub et al. 1990; Harvey, Gallagher et al. 1998). The first-derivative was then taken from the fit to determine $\mathrm{dn} / \mathrm{dT}(\mathrm{T})$ for five different wavelengths as shown in Fig. 4.4.

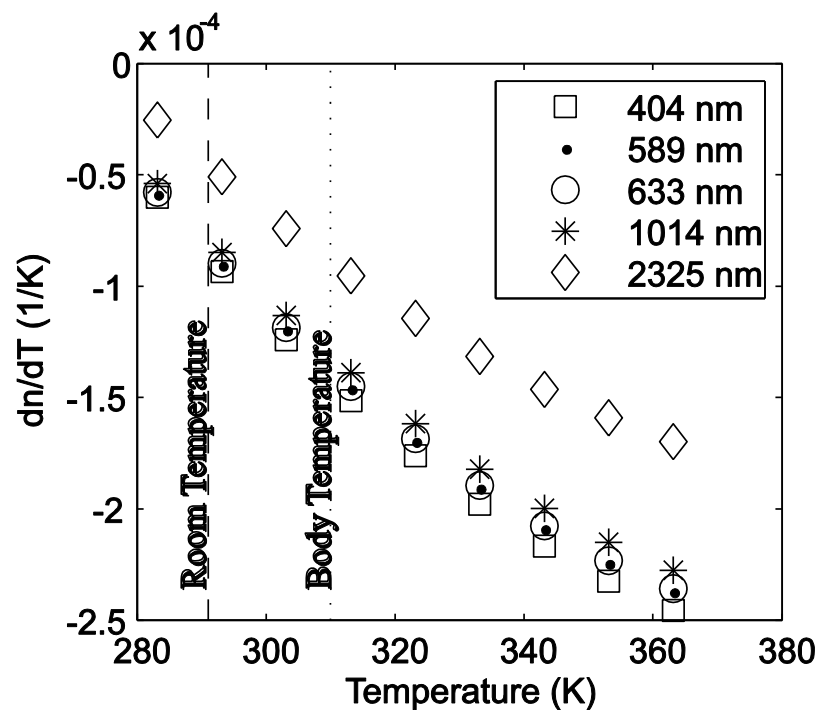

Figure 4.4: Values of $\mathrm{dn} / \mathrm{dT}(1 / \mathrm{K})$ as a function of temperature for water in the liquid phase for five different wavelengths. Values here were based upon taking the derivative of $n(\mathrm{~T})_{\mathrm{P}}$ at each of the five wavelengths as reported in literature at $0.1 \mathrm{MPa}$ (Schiebener, Straub et al. 1990; Harvey, Gallagher et al. 1998).

\subsection{RESULTS}

\subsubsection{Model Compared to Experimental Z-Scan Data}

By substituting Eq. 4.17 into Eq. 4.7 and using the $\mathrm{ABCD}$ beam-propagation method described in Eq. 4.8-4.10, the model was arranged to simulate the z-scan set up depicted in Fig. 3.2. A comparison of the results from this portion of the model to experimental z-scan data for varying input power and a 10-mm thick water-filled cuvette at room temperature can be found in Fig.'s 4.5 and 4.6. 
(a)

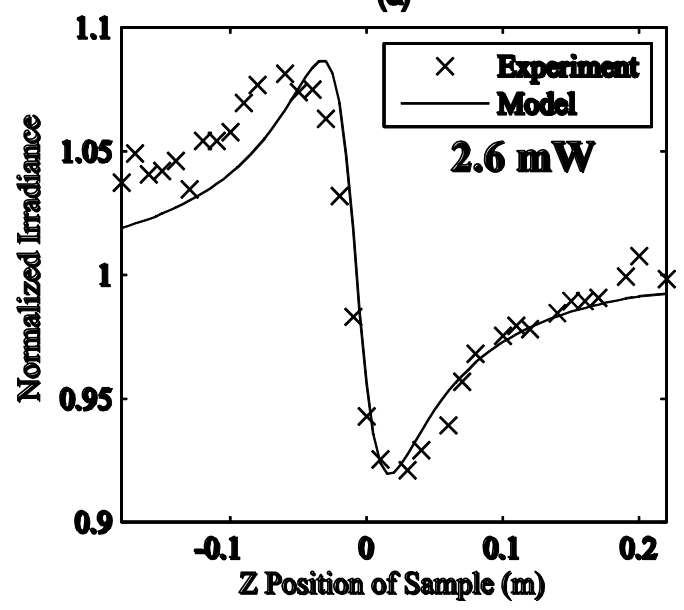

(b)

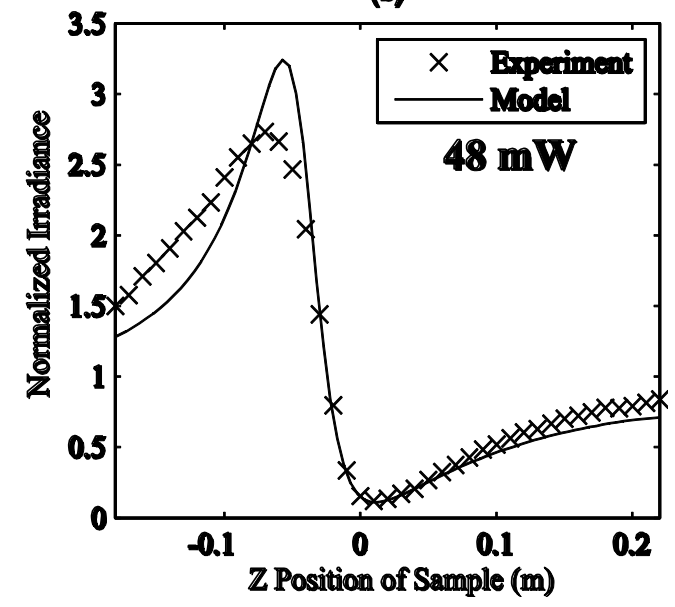

Figure 4.5: Z-scan data for a 10-mm water-filled cuvette exposed to 1313-nm CW laser source for $1 \mathrm{~s}$ at (a) $2.6 \mathrm{~mW}$ and (b) $48 \mathrm{~mW}$..

(a)

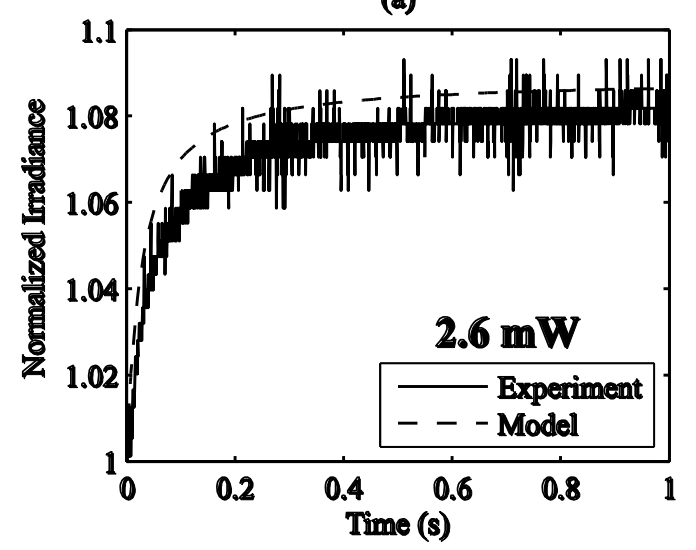

(c)

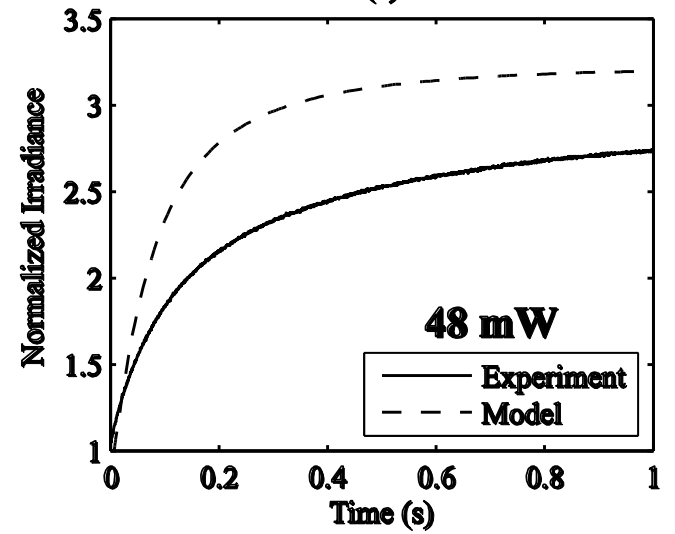

(b)

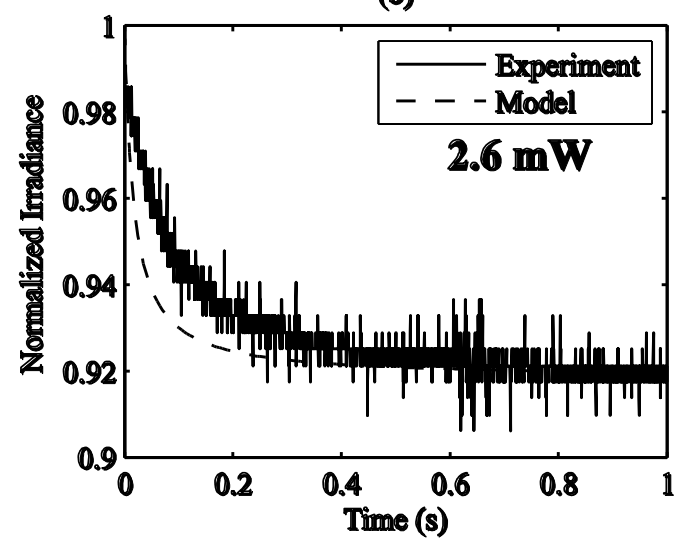

(d)

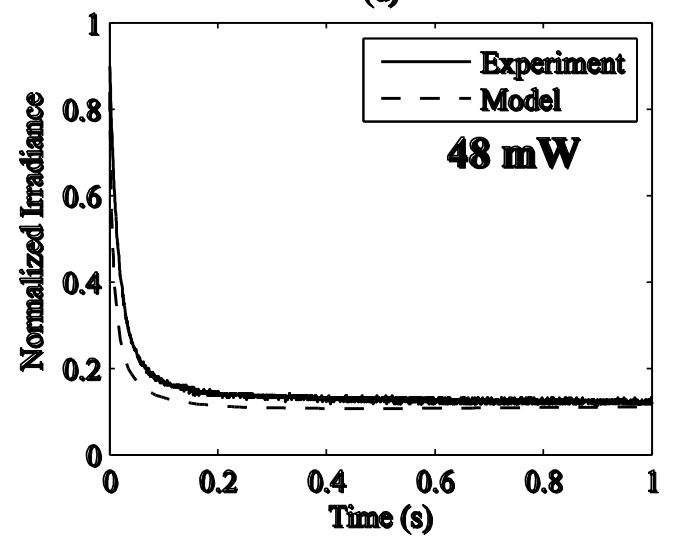

Figure 4.6: The transient response for the z-scan peaks from Fig. 4.5 for $2.6 \mathrm{~mW}$ (a) maximum and (b) minimum and $48 \mathrm{~mW}$ (c) maximum and (d) minimum. 
A bulk value of $-4.46^{*} 10^{-5} \mathrm{~K}^{-1}$ for $\mathrm{dn} / \mathrm{dT}$ was found to give the best fit to the experimental 1313-nm radiation z-scan data in terms of line width before and after the focal position at $\mathrm{z}=0$ and yielded the best approximation to the slope between the maxima and minima. A detailed discussion of the impact of the value of $\mathrm{dn} / \mathrm{dT}$ is reserved for the discussion.

\subsubsection{Thermal Lensing Model Trends in the Virtual Eye}

The $\mathrm{dn} / \mathrm{dT}$ value found to fit the z-scan data was used in the simple eye model presented in Fig. 4.3 to make predictions on the trends of thermal lensing in a human eye. Analysis of data from Schiebener, Straub et al. 1990, and Harvey, Gallagher et al. 1998, (Fig. 4.4) suggests that this value of $\mathrm{dn} / \mathrm{dT}$ will approximately double when the ambient temperature is increased from room to body temperatures. To test for the significance in this variation due to an increasing thermo-optic coefficient in our model, we used a constant $\mathrm{dn} / \mathrm{dT}$ value of $-4.46^{*} 10^{-5} \mathrm{~K}^{-1}$ for the results in Fig.'s $4.7-4.10$ and then doubled $\mathrm{dn} / \mathrm{dT}$ to $-8.92 * 10^{-5} \mathrm{~K}^{-1}$ for the results in Fig. 4.11. Again, Eq. 4.17 was substituted into Eq. 4.7 and the ABCD beam-propagation method described in Eq. 4.84.10 was used, only now the model was arranged to simulate the virtual eye (Fig. 4.3). All other values for the simple eye model were previously described.

The influence of power from a Gaussian, 1318-nm laser radiation source input into the eye on the beam's radius at the retina as a function of time is shown in Fig. 4.7. The results in Fig. 4.7 and Fig.'s 4.9 - 4.11 were normalized to the initial, $\mathfrak{t}=0$, beam radius at the retina in order to examine the magnitude of the increase in the beam's radius (i.e. - a factor of two, ten, etc.) due to thermal lensing. Where appropriate, the value for normalizing the results is given in the caption. The $20-\mathrm{W}$ case, shown in Fig. 4.7, 
represents the upper limit of the 1318-nm, CW laser radiation source which was used in CW non-human primate retinal damage studies conducted by Zuclich, Lund et al. 2004.

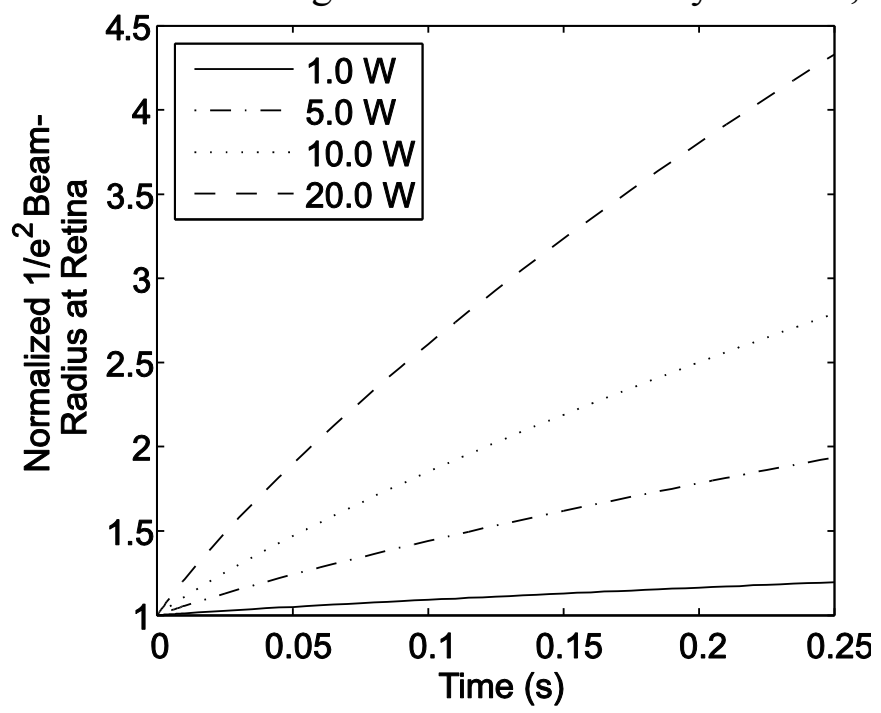

Figure 4.7: Increase in the beam radius at the retina as a function of time for selected powers delivered to the cornea. Parameters were for 1318-nm, CW laser radiation into a human eye, with a $1 / \mathrm{e}^{2}$ Gaussian beam radius of $2.12 \mathrm{~mm}$ at the virtual cornea. The yaxis was normalized to $98 \mu \mathrm{m}$, which was the beam radius at the virtual retina determined at time $=0 \mathrm{~s}$.

Keeping the power delivered to the cornea at $3 \mathrm{~W}$, the influence of the beam's diameter from a Gaussian, 1318-nm laser radiation source input at the cornea on the beam's radius at the retina as a function of time is shown in Fig. 4.8. Parameters were for a $3-\mathrm{W}, \mathrm{CW}, 1318-\mathrm{nm}$ laser radiation source delivering collimated, 1/e $\mathrm{e}^{2}$ Gaussian beam radii of $0.5,0.75,1.25,1.75$ and $2.25 \mathrm{~mm}$ at the virtual cornea. Note that Zuclich, Lund et al. 2004, reported the corneal $\mathrm{ED}_{50}$ for a CW, 1318-nm laser radiation delivered to the cornea for 0.28-s exposures is $0.56 \mathrm{~J}$. Zuclich, Lund et al. 2004, used a beam-radius of $0.5 \mathrm{~mm}$ at the cornea, giving an $\mathrm{ED}_{50}$ of approximately $72 \mathrm{~J} / \mathrm{cm}^{2}$. The point in time which each of the $0.5,0.75,1.25,1.75$ and 2.25 -mm beam-radii at the cornea begins to exceed the $72 \mathrm{~J} / \mathrm{cm}^{2}$ corneal $\mathrm{ED}_{50}$ is at $0.19,0.42,1.18,2.31$, and $3.82 \mathrm{~s}$ respectively. 


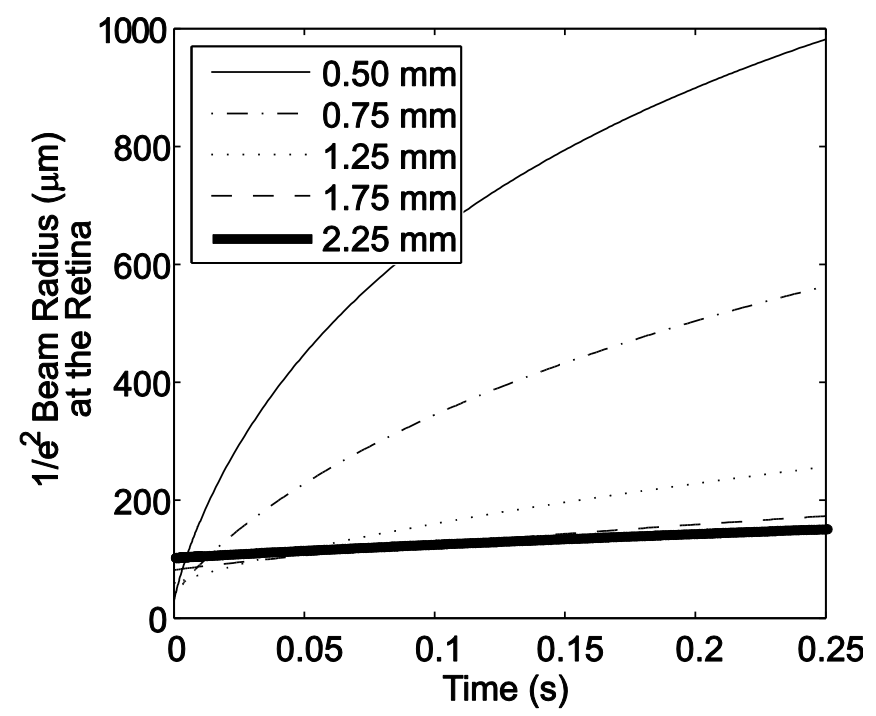

Figure 4.8: Increase in the beam radius at the retina as a function of time for selected input beam radii at the cornea.

Model results for short and long-time intervals are shown for 1318 and 1150-nm, CW laser radiation for various powers delivered to the cornea in Fig.'s $4.9-4.11$. Parameters were for 1318 and 1150-nm, CW laser radiation, in Fig.'s 4.9 - 4.11 and Fig. 4.10 respectively, into a human eye with a $1 / \mathrm{e}^{2}$ Gaussian beam-radius of $2.12 \mathrm{~mm}$ at the virtual cornea. Unless specifically stated in the caption, all parameters in the remaining model results were the same, save for the absorption coefficients and index of refraction due to dispersion. In Fig. 4.11, all parameters were identical to those used to produce the model results in Fig. 4.9, only the value of $\mathrm{dn} / \mathrm{dT}$ was doubled from $-4.46^{*} 10^{-5} \mathrm{~K}^{-1}$ to $8.92 * 10^{-5} \mathrm{~K}^{-1}$. 
(a)

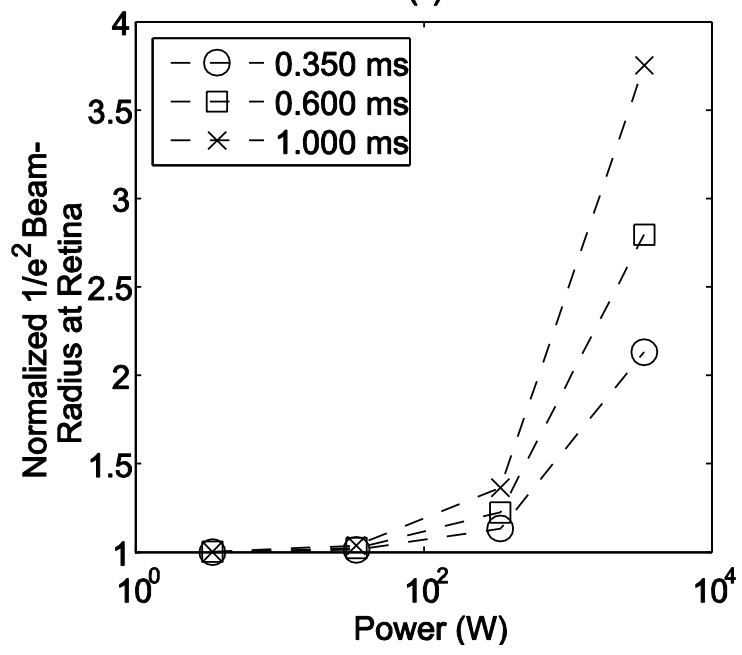

(b)

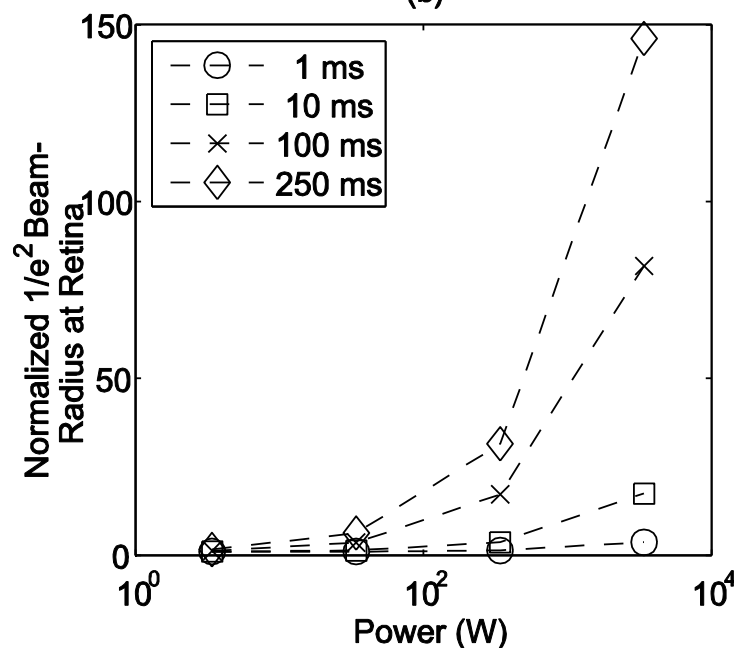

Figure 4.9: Increase in the beam-radius for 1318-nm laser radiation at the retina as a function of input power at the cornea for (a) short time $(\mathrm{t} \leq 1 \mathrm{~ms})$ and (b) long time (1 ms $<\mathrm{t} \leq 250 \mathrm{~ms}$ ). Note the $340-\mathrm{W}$ power at $0.350 \mathrm{~ms}$ is approximating the $\mathrm{ED}_{50}$ threshold level reported in Zuclich, Lund et al. 2004, for the equivalent wavelength. The y-axis was normalized to the initial beam radius of $98 \mu \mathrm{m}$.

(a)

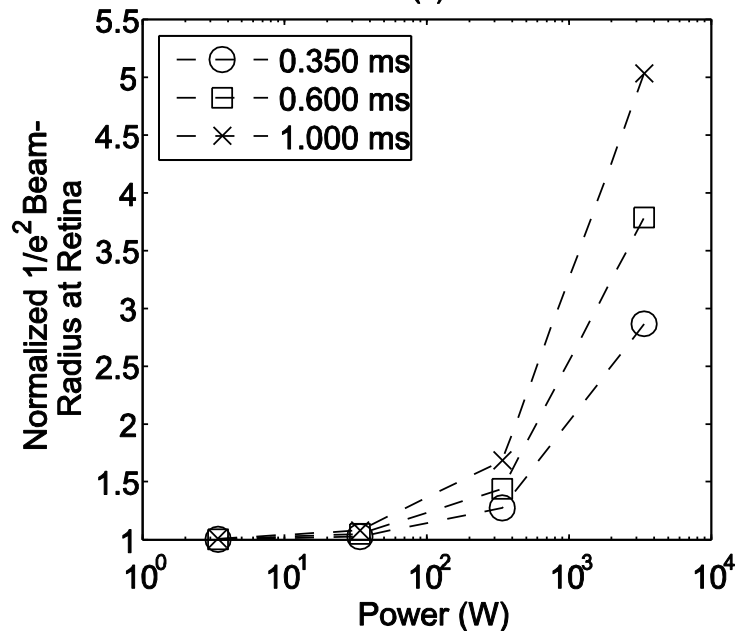

(b)

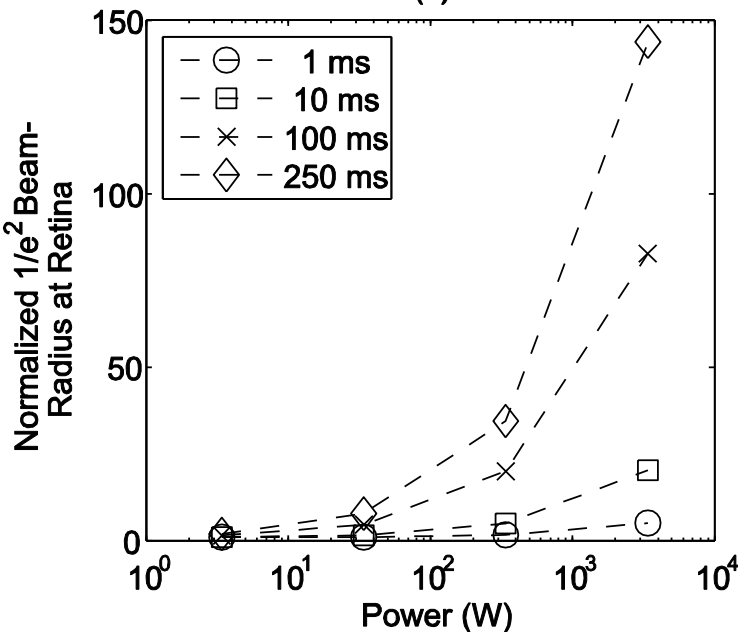

Figure 4.10: Increase in the beam-radius for 1150-nm laser-radiation at the retina as a function of input power at the cornea for (a) short time ( $\mathrm{t} \leq 1 \mathrm{~ms})$ and (b) long time (1 ms $<\mathrm{t} \leq 250 \mathrm{~ms}$ ). The $\mathrm{y}$-axis was normalized to the initial beam radius of $82 \mu \mathrm{m}$. 
(a)

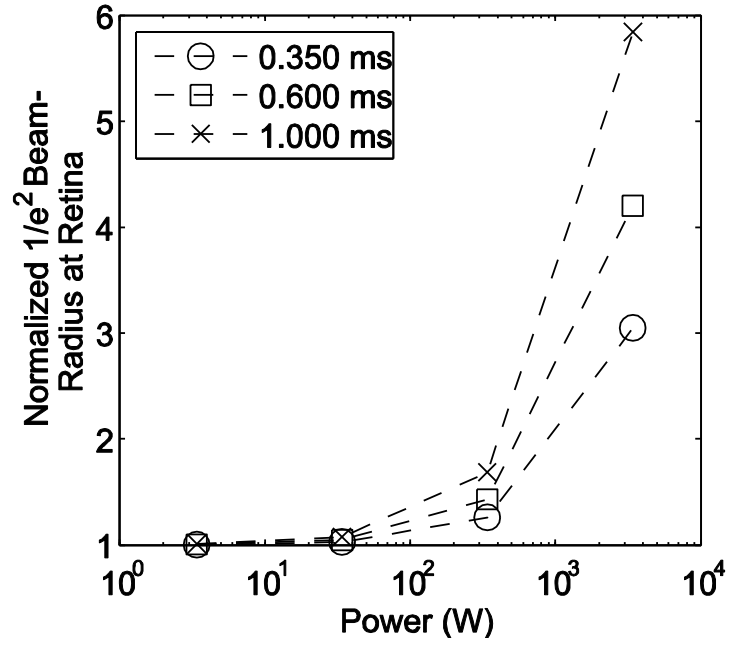

(b)

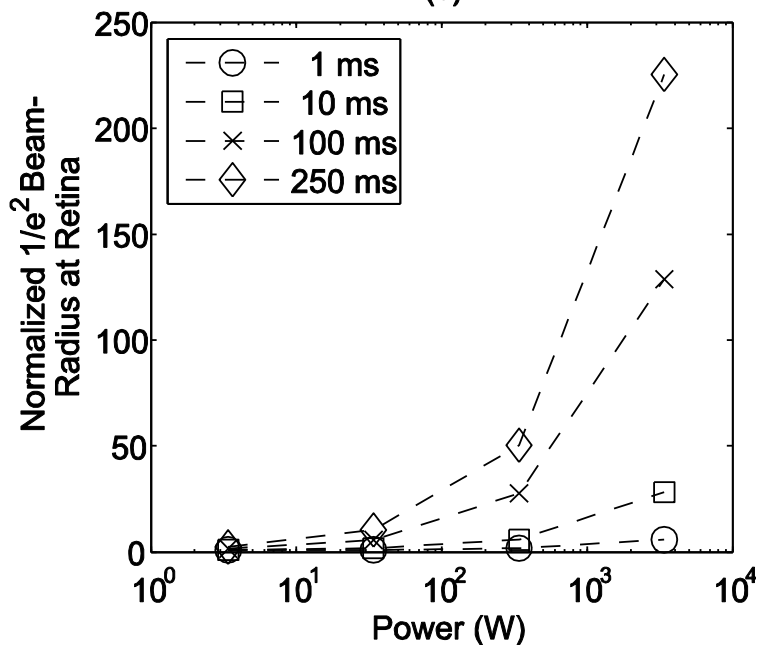

Figure 4.11: Increase in the beam-radius for 1318-nm laser radiation at the retina as a function of input power at the cornea for (a) short time ( $\mathrm{t} \leq 1 \mathrm{~ms})$ and (b) long time (1 ms $<\mathrm{t} \leq 250 \mathrm{~ms})$ having doubled the value of $\mathrm{dn} / \mathrm{dT}$ to $-8.92 * 10^{-5}\left(\mathrm{~K}^{-1}\right)$. The $\mathrm{y}$-axis was normalized to the initial beam radius of $98 \mu \mathrm{m}$.

\subsection{DISCUSSION}

Analysis of the Schiebener, Straub et al. 1990, and Harvey, Gallagher et al. 1998, data for $\mathrm{n}(\mathrm{T}, \lambda, \mathrm{P})$ revealed three trends for water in the liquid phase between 283 and $363 \mathrm{~K}$ at pressures from 0.1 to $0.2 \mathrm{MPa}$ (see Fig. 4.4 for $\mathrm{dn} / \mathrm{dT}$ at $0.1 \mathrm{MPa}$ ). First, the modulus of the thermo-optic coefficient, $|\mathrm{dn} / \mathrm{dT}|$, decreases with increasing wavelength for laser radiation between 400 and $2325 \mathrm{~nm}$. Second, the modulus of the thermo-optic coefficient $\mathrm{dn} / \mathrm{dT}$ increases as temperature increases for liquid water and finally, decreases very slightly as pressure increases from 0.1 to $0.2 \mathrm{MPa}$. This suggests the magnitude of $\mathrm{dn} / \mathrm{dT}$ for ocular media at body temperature and standard ocular pressures should be approximately two times greater than $\mathrm{dn} / \mathrm{dT}$ values for water held in a cuvette at room temperature, (see vertical lines in Fig. 4.4). Understanding these fundamental trends will lend insight into predicting thermal lensing trends in the eye. 
Using data from Schiebener et al. as a guide, an estimate for $\mathrm{dn} / \mathrm{dT}$ at a wavelength of $1313 \mathrm{~nm}$ between 290-293 K (room temperatures) and pressures near 1 atm $(\sim 0.1 \mathrm{MPa})$ was found to be between $-6.96 * 10^{-5}$ and $-7.86^{*} 10^{-5} \mathrm{~K}^{-1}$ (see Fig. 4.4). The $\mathrm{dn} / \mathrm{dT}$ value of $-4.46^{*} 10^{-5} \mathrm{~K}^{-1}$ determined to yield the best fit to $\mathrm{z}$-scan data comes close to this range of $\mathrm{dn} / \mathrm{dT}$ values. The error associated with the selected value for $\mathrm{dn} / \mathrm{dT}$ is very difficult to quantify given the sensitivity of the z-scan data being dependent upon the optical alignment of the system and aperture size. Misalignment in the optical system for the z-scan would introduce aberrations not accounted for in the first-order model which only considers a Gaussian wavefront. Further, it has been demonstrated that thermal lensing in water results in convective flow processes introducing non-linear aberrations into the steady-state reading (Buffett and Morris 1983; Motamedi, Welch et al. 1988; Alfonso, Revert et al. 1990; Vincelette, Thomas et al. 2007).

Though the standard deviation of the normalized intensity for the experimental zscan measurements was +/- 0.02 arbitrary units, there are limitations of the closedaperture z-scan. Measures were taken to minimize errors to include routine optical alignment checks before every data collection and careful knife edge measurements to characterize the laser-beam's profile. However meticulous these measures were, they cannot account for the uncertainty with the beam's deflection due to convectional effects projected onto a stationary aperture. Convection causes a non-linear disruption in the temperature profile, making mathematical solutions based upon radially symmetric profiles invalid. The strength of this non-linear process has been shown to be directly related to the exposure length, power of the radiation source and absorptivity of the exposed media. With enough heat generation the Gaussian beam loses its symmetry, which a closed-aperture z-scan cannot account for or detect (note the donut shapes 
observed in Chapter 3 after 1-s exposures in Fig. 3.9). For this reason, z-scan data were collected for low (2.6-mW) and high (48-mW) power. Alfonso, Revert et al. 1990, reported using a thin vertical slit in place of the iris aperture presented in Fig. 4.2 to overcome this problem, however, slit length is constrained by the physical dimensions of the selected laser-radiation detector.

The strength in the formation of the non-linear beam profile has been shown to be directly related to the exposure length, power of the radiation source and absorptivity of the exposed media (Buffett and Morris 1983; Motamedi, Welch et al. 1988; Alfonso, Revert et al. 1990; Vincelette, Thomas et al. 2007). For this reason, z-scan data were collected for low $(2.6-\mathrm{mW})$ and high $(48-\mathrm{mW})$ power. The condition for a symmetric beam profile is more likely to be maintained in the low power data set. Comparing the low to high power z-scan data (Fig. $4.5 \mathrm{a}$ and $\mathrm{b}$ respectively), it is evident the amount of over-estimation by the model in the low-power is much less than in the high-power data set. This is expected since the first-order thermal lensing model, FOTLM, uses the paraxial approximation which has been reported to be limited to cases where radial symmetry is maintained (Kovsch, Hagan et al. 1999). The model in the low-power transient result (Fig. 4.6 a) over estimated the z-scan irradiance data by less than 1\% compared to the more than $18 \%$ over estimated by the model in the high-power data set at 1 second (Fig. $4.6 \mathrm{c}$ ). It is likely that radial symmetry is not maintained experimentally in the higher power case, but the FOTLM assumes radial symmetry is maintained helping to explain some of this over-estimation. The low-power z-scan data appear noisier than the high-power data due to the fact the irradiance levels were dipping towards the lower limit of the detector. When the limitations of the single-beam closed-aperture z-scan are considered, the results from the first-order, thermal-lensing, z-scan model suggests our 
model provides a good estimate for the strength of the thermal lens effect. The $\mathrm{dn} / \mathrm{dT}$ value of $-4.46^{*} 10^{-5} \mathrm{~K}^{-1}$ found in the fit is a reasonable estimate to trends in thermal lensing effects in the eye. With the consideration of imperfections in the optical system and the formation of asymmetric beam profiles, it is prudent to discuss the uncertainty associated with our model's results.

The first-order model's limitations include that radial symmetry is assumed to be maintained throughout the exposure, neglect heat conduction along the z-axis and treat the eye as a simple system of homogenous slabs with a single curved surface placed on the cornea. The creation of the asymmetric temperature and non-linear beam profiles mean our mathematical solution based upon radially symmetric profiles becomes invalid. The use of the simple eye model affords a straight-forward propagation of the beam profile, reducing the complexity of an already difficult heat conduction problem in the creation of the thermal lens. The first-order thermal lensing results from the simple eye assist in approximating the magnitude the thermal lens may have on the size of the laserbeam's radius at the retina in an eye exposed to CW NIR laser radiation. It should be stressed that the eye is a more complex optical system than the simple model presented here. Advanced models of the eye can be found in literature (Artal, Benito et al. 2006; Fernandez, Unterhuber et al. 2006; Tabernero, Piers et al. 2006).

More advanced models of thermal lensing in ocular media to include heat conduction along the z-axis and the non-linear beam profile are currently underway at the Air Force Research Laboratory at Brooks City-Base. As the fidelity of the thermal lensing model is increased, the complexity and computational needs, to include resources and time, of the model are increased. The first-order model is computationally inexpensive and fits our z-scan data reasonably well, particularly for the low-power z- 
scan experimental data in Fig.'s 4.5a and 4.6a. Regardless of the first-order model's limitations, I find it suitable for assessing trends of thermal lensing in the eye caused by near-infrared laser radiation.

From model results shown in Fig. 4.7, it is evident the magnitude of thermal lensing increases with power. For strongly absorbed wavelengths, the fluence at the retina can vary significantly with input power and time of the exposure. The power levels presented in Fig. 4.7 were chosen to represent the range of power, up to the limit of, the CW 1318-nm laser source used in the retinal damage studies conducted by Zuclich, Lund et al. 2004. Note at $20 \mathrm{~W}$ under the simulated conditions, the beam's $1 / \mathrm{e}^{2}$ radius doubles from $98 \mu \mathrm{m}$ to $196 \mu \mathrm{m}$ at approximately $56 \mathrm{~ms}$ and is more than four times larger than initial state after $250 \mathrm{~ms}$. Retinal damage threshold studies, summarized in section 2.1, were never successful on primates for CW 1318-nm and it was never understood as to what the mechanisms were which circumvented damage (Vincelette, Rockwell et al. 2008). Model results from Fig. 4.7 suggest that the fluence rate delivered to the retina would decrease over time, potentially preventing damage to the retina.

The input beam diameter significantly increases the thermal lensing effect as illustrated in Fig. 4.8. The trend reveals as a constant power Gaussian input beam decreases in radius at the cornea the magnitude of the thermal lens greatly increases as a result of the increased heat-source along the optical axis. This is not surprising given the physics of the z-scan. The most dramatic impact of the thermal lens occurs inside the focus and is even described as the reason for creating z-scan systems where the sample is shorter than the depth of focus of the system (Franko and Tran 1996). Thus, as the depth of focus (DOF) increases, the magnitude of the thermal lens inside the media should 
increase as well. The DOF is given as $2 \pi \lambda *\left(\mathrm{f}_{\lambda} / 2 \mathrm{~d}\right)^{2}$, where $\mathrm{f}_{\lambda}$ is the focal length of the virtual eye for the respective wavelength, $\lambda$, and $d$ is the input diameter of the laser beam at the virtual cornea. Initially, at time $=0 \mathrm{~s}$, the smaller input radii delivered to the cornea produce the smallest laser-spot at the virtual retina. At some point in time, the conditions with the longer DOF at the virtual retina will give the larger thermal lens. This suggests that when calculations are made to increase the fluence delivered to the retina for strongly-absorbed laser radiation, the condition of decreasing the input laser beam radius may in fact be decreasing the delivered fluence due to the thermal lens.

Zuclich, Lund et al. 2004, and Zuclich, Lund et al. 2007, reported for a 1315-nm wavelength with a $350-\mu$ s retinal exposure, the $\mathrm{ED}_{50}$ is $0.844 \mathrm{~J} / \mathrm{cm}^{2}$ delivered at the retina. Taking this value and scaling it to the $1 / \mathrm{e}^{2}$ beam radius of $2.1 \mathrm{~mm}$ indicates that $0.119 \mathrm{~J}$ was delivered to the cornea. Dividing by an exposure time of $350 \mu$ s provides an average power $340 \mathrm{~W}$. A series of powers input at the cornea for four different magnitudes, including this $340-\mathrm{W}$ case, are shown in Fig. 4.9. It takes only $3 \mathrm{~ms}$ before the beam's radius at the virtual retina doubles in size. As power increases, the thermal lens will occur faster than for lower power. Zuclich, Lund et al. 2004, were able to determine retinal damage thresholds for short-pulsed $(<1 \mathrm{~ms}), 1318-\mathrm{nm}$ laser radiation since the time for the thermal lens to take hold is significantly longer than the pulse duration of the laser source.

In Fig.'s 4.9 and 4.10, the model was run for four different orders of magnitude in input power at the cornea for 1318 and 1150-nm laser radiation wavelengths respectively to demonstrate how the change in absorption and depth of focus impacts the thermal lens. The absorption coefficients for the components of the eye in Fig. 4.3 for 1150 and 1318$\mathrm{nm}$ laser radiation are given in Table 4.2. Less than $2 \%$ of the 1318 -nm radiation reaches 
the virtual retina compared to the near 7\% energy transmitted at $1150 \mathrm{~nm}$ (see Chapter 2) (Vincelette, Rockwell et al. 2008). Given the trends in literature from Schiebener, Straub et al. 1990, for $\mathrm{dn} / \mathrm{dT}$, the value of $\mathrm{dn} / \mathrm{dT}$ should be slightly greater for $1150 \mathrm{~nm}$ compared to $1318 \mathrm{~nm}$. Even with the value of $\mathrm{dn} / \mathrm{dT}$ being the same for both wavelengths, these results can lend information into the behavior of the thermal lens. Upon observation of Fig.'s 4.9 and 4.10, the 1150-nm radiation begins to thermally lens more strongly for points in time less than $250 \mathrm{~ms}$, but at the 250 -ms time point, the magnitude of the thermal lens is very close to the model results of the 1318-nm radiation case. Overall, the results in Fig.'s 4.9 and 4.10 suggest for these conditions (i.e. - input beam diameter and power at the corneal plane) there is no large variation in the degree of thermal lensing between 1318 and 1150-nm laser radiation.

Fig. 4.11 illustrates the impact in increasing the value of the $\mathrm{dn} / \mathrm{dT}$ modulus. The simulations run for Fig.'s 4.9 and 4.11 were identical save for the fact that $\mathrm{dn} / \mathrm{dT}$ was $4.46^{*} 10^{-5}$ and $-8.92 * 10^{-5} \mathrm{~K}^{-1}$ respectively. Doubling the magnitude of $\mathrm{dn} / \mathrm{dT}$ caused the $1 / \mathrm{e}^{2}$ beam-waist radius results in Fig. $4.11 \mathrm{~b}$ to be approximately $33 \%$ larger at $250 \mathrm{~ms}$ than the comparable results in Fig. 4.9b. These results demonstrate that, all things being equal, the magnitude of the thermal lens does increase, but is not proportional with the modulus of $\mathrm{dn} / \mathrm{dT}$.

\subsection{CONCLUSION}

The mathematical model was found to fit experimental z-scan data reasonably well for a dn/dT value of $-4.46^{*} 10^{-5}\left(\mathrm{~K}^{-1}\right)$ for water at room temperature exposed to 1313 $\mathrm{nm}, \mathrm{CW}$ laser radiation. This value is close to the predicted $\mathrm{dn} / \mathrm{dT}$ values based on analysis of the Schiebener, Straub et al. 1990, and Harvey, Gallagher et al., 1998, data 
(Fig. 4.4). The trends from Fig. 4.4 suggest the value of $\mathrm{dn} / \mathrm{dT}$ for water at body temperature are approximately two times greater than the $\mathrm{dn} / \mathrm{dT}$ value for water at room temperature. This conservative value of $\mathrm{dn} / \mathrm{dT},-4.46^{*} 10^{-5}\left(\mathrm{~K}^{-1}\right)$ lends information on trends of thermal lensing in the human eye exposed to CW laser radiation in the 11501350-nm region. The model provides a reasonable first-order approximation on the trends of the thermal lensing effect on the fluence rate delivered to the retina. The thermal lens causes the fluence rate delivered to the retina would decrease over time, potentially preventing damage to the retina for NIR exposures when sufficient time is allowed for thermal lensing to occur. Modeling results show the thermal lens take several ms to develop. Retinal damage studies conducted by Zuclich, Lund et al. 2004, on non-human primates were successful for exposure durations less than a ms, but could never cause a retinal lesion for exposures greater than $100 \mathrm{~ms}$. Based on the modeling results, the CW 1318-nm laser radiation retinal damage studies for exposures greater than $100 \mathrm{~ms}$ may have been impacted by a decreasing delivered retinal fluence rate precluding damage.

\subsection{ACKNOWLEDGEMENTS}

I would like to thank the following people for their assistance with the data collection and/or analysis presented in this chapter:

Dr. Robert J. Thomas from the Air Force Research Laboratory (AFRL), Optical Radiation Branch, and Capt. Dustin Mixon from AFRL, Radar Branch.

Mr. Gary Noojin and Mr. Clifton D. Clark with Northrop Grumman.

Dr. Taufiquar Khan, from Clemson University. 


\section{CHAPTER 5}

\section{TRENDS IN RETINAL DAMAGE THRESHOLDS FROM CONTINUOUS-WAVE NEAR-INFRARED LASER RADIATION: a study at 1110, 1130, 1150 and $1319 \mathrm{~nm}$}

\subsection{ABSTRACT}

In this chapter, the retinal damage thresholds for continuous wave, $\mathrm{CW}$, nearinfrared, NIR, laser radiation for wavelengths at 1110, 1130, and 1319-nm were obtained and then predicted for all NIR wavelengths between 1100-1350-nm. Using $1 / \mathrm{e}^{2}$ beam diameters of 4.8-5 $\mathrm{mm}$ at the cornea, the experimental retinal damage threshold, $\mathrm{ED}_{50}$, for 100-ms exposures for laser radiation wavelengths at 1110, 1130 and 1319-nm were determined to be 193,270 and $13713 \mathrm{~mW}$ delivered to the cornea respectively. The retinal damage threshold for the 1150-nm wavelength was statistically undetermined due to laser-power limitations, but was achieved in 1 out of 3 subjects tested. Using trends of transmitted energy to the retina, refractive error of the eye and linear absorption of the retina, a scaling factor (SF) method was created to fit the experimental data, predicting retinal damage thresholds over the 1100-1350-nm region. The content in this chapter has been accepted for publication in Vincelette, Rockwell et al., Lasers in Surgery and Medicine, 2009.

\subsection{INTRODUCTION}

As detailed in Chapter 2, continuous-wave, CW, near-infrared, NIR, (> 1100) laser radiation retinal damage thresholds have never been achieved in conventional rhesus subjects (see section 2.1). For review, the formation of a lesion from CW NIR 
laser radiation is related to three fundamental physical characteristics: 1 - the transmission of laser radiation to the retina, 2 - absorption of laser radiation by the retina and pigment epithelium and 3 - the diameter of the laser-spot formed at the retina. The diameter of the laser-spot formed at the retina is typically taken to be dependent on the natural chromatic dispersion properties of the eye, independent of absorption and thermal properties of the tissue.

This Chapter presents data from a retinal damage threshold study using 1110, 1130, 1150 and 1319-nm continuous-wave near-infrared laser radiation. The paramacula was exposed for laser radiation wavelengths of 1110, 1130 and 1150-nm, while the macula was used for the 1319-nm study. These data are compared to trends in the three fundamental criteria previously mentioned and contrasted to the NIR CW 1318-nm laser radiation results found in earlier retinal threshold studies.

\subsection{BACKGROUND}

In the CW 1318-nm NIR laser radiation retinal damage threshold studies conducted in rabbits by Zuclich and Lund et al., lesions were never apparent after onehour post exposure, but were seen after 24-hours (Zuclich, Lund et al. 2004; Zuclich, Lund et al. 2007). In other laser-radiation retinal damage studies, below $1100 \mathrm{~nm}$, a small number of lesions appeared at the 24-hour end point, but the majority $(>90 \%)$ of lesions were present at 1-hour (Cain, Noojin et al. 2003). Histology of the 1318-nm radiation lesions also revealed full-retinal thickness damage at threshold level energies, a trait seen in visible lesions only for suprathreshold exposures. Zuclich and Lund et al. 2004, also reported that lesions which appeared at 24-hours increased, even doubling, in size, stabilizing after 48-hours post exposure. Again, extensive growth in lesion size is 
not a characteristic trait when visible laser radiation is used to create retinal lesions. Though histology is not provided for the study presented here, these observations from Zuclich and Lund et al. 2004, provide the basis for understanding our data.

Lund, Edsall et al. 2008, have created an action spectrum to fit retinal damage threshold data using three fundamental criteria of transmission, absorption and the radius, subject to chromatic dispersion of the laser radiation reaching the retina. The transmission of NIR laser radiation from the cornea to the retina in a rhesus (Macaca mulatta) and human was predicted using one-dimensional Beer's law and linear absorption data from Maher 1978. Physical thicknesses for the cornea, aqueous humor, crystalline lens and vitreous humor were obtained from literature and were detailed in Fig. 2.5 (Westheimer 1980; Li, Zwick et al. 2000; Fernandes, Bradley et al. 2003). The percent transmission decreases sharply, as indicated in Fig. 5.1, for the three wavelengths of 1110,1130 and $1150-\mathrm{nm}$ from 57.3 to 42.2 and finally $12.4 \%$ respectively. The percent of laser radiation transmitted at $1319-\mathrm{nm}$ for a rhesus eye is $\sim 3 \%$. The total absorption of the pre-retinal media can be estimated by subtracting the percent transmission from $100 \%$ (this neglects reflection). 


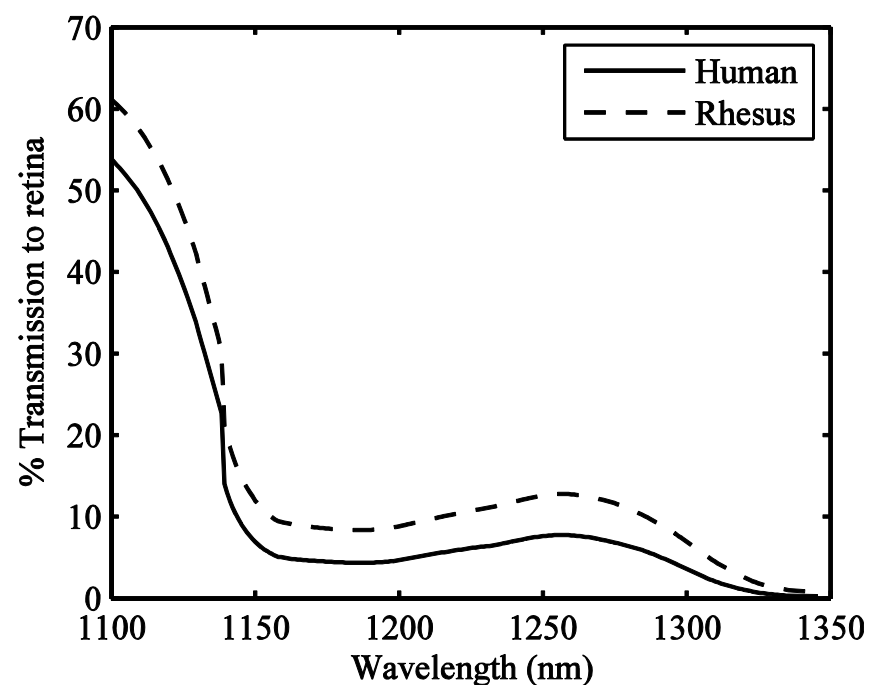

Figure 5.1: Transmission of laser radiation in a human and rhesus eye based on linear absorption data of Maher 1978. These results assumed physical thicknesses of the cornea, aqueous humor, crystalline lens and vitreous humor from literature (Westheimer 1980; Li, Zwick et al. 2000; Fernandes, Bradley et al. 2003).

Lund, Edsall et al. 2000, determined the retinal pigment epithelium, RPE, laser radiation absorption data which provided a best-fit to retinal damage threshold data were from Gabel, Birngruber, et al. 1976, Gabel, Birngruber et al. 1978, and Birngruber, Hillenkamp et al. 1985, where the percent of laser radiation reaching the retina is absorbed by the RPE, at 1110, 1130,1150 and 1319 -nm wavelengths is $3.87 \%, 3.55 \%$, $3.26 \%$ and $1.58 \%$ respectively. Note the variability between the 1110,1130 and $1150-$ nm wavelengths is less than $1 \%$, but the $1319-\mathrm{nm}$ wavelength is about a factor of 2.25 times smaller (Table 5.1). Birngruber, Hillenkamp et al. 1985 compared the histological sections of a monkey's retina for several subjects, reporting that the average thickness of the actual pigmented granules which are found in the cells of the RPE is approximately 5 $\mu \mathrm{m}$. Birngruber, Hillenkamp et al. 1985 reported finding equivalent pigmentations in the RPE and choroid with pigmentation distributed throughout the choroid (no pigmentation was reported in the choriocapillaris). From their histological findings, Birngruber, 
Hillenkamp et al. 1985 reported the absorption coefficient, $\mu_{\mathrm{a}}$, of the pigmented portion of the RPE to be the same for the pigmented portion of the choroid. Here, this is assuming the absorption coefficient for RPE and choroid reported by Birngruber, Hillenkamp et al. 1985 includes water absorption. In areas of the retina where Birngruber, Hillenkamp et al. 1985 report an absence of pigmentation, it is assumed that the absorption coefficient is the same as water. The $\mu_{\mathrm{a}}$ for RPE over wavelengths from 1000-1400 nm is shown in Fig. 5.2. The exponential fit to the absorption coefficients, $\mu_{\mathrm{a}}$ $\left[\mathrm{cm}^{-1}\right]$, for wavelength, $\lambda[\mathrm{nm}]$, in rhesus RPE is given as

$$
\mu_{\mathrm{a}}(\lambda)=15874 * \exp \left(-4.59 * 10^{-3} \lambda\right)
$$

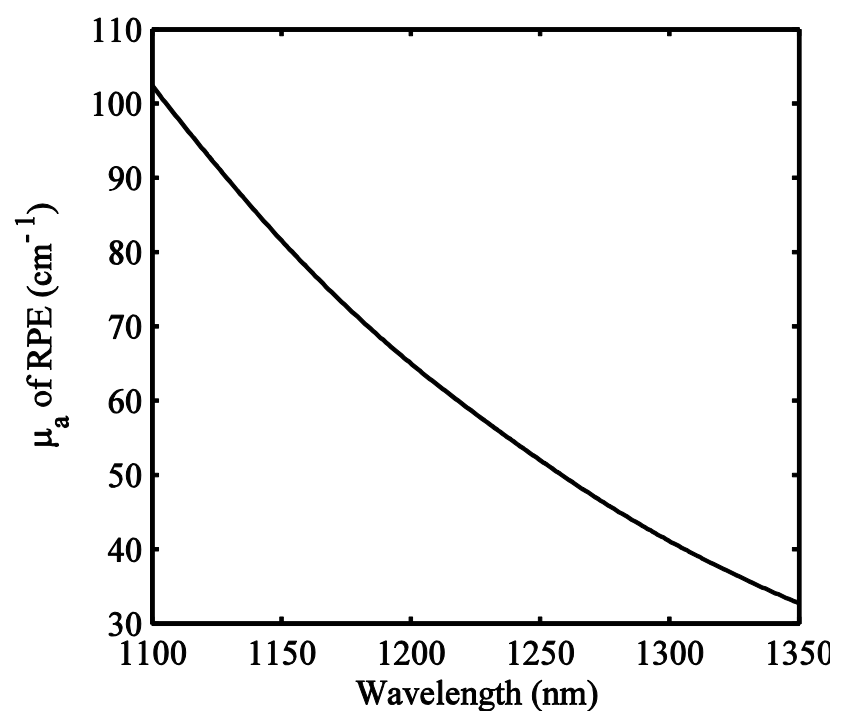

Figure 5.2: Absorption by retinal pigment epithelium, RPE, based on a fit to data from Birngruber, Hillenkamp et al. 1985.

Table 5.1: The linear absorption coefficient of the RPE, $\mu_{\mathrm{a}}$, for the laser radiation wavelengths in this study.

\begin{tabular}{|c|c|}
\hline$\lambda(\mathrm{nm})$ & $\mu_{\mathrm{e}}\left(\mathrm{cm}^{-1}\right)$ \\
\hline 1110 & 97.87 \\
1130 & 89.30 \\
1150 & 81.48 \\
1319 & 37.54 \\
\hline
\end{tabular}


When a collimated beam is normal to the cornea, it is focused by the cornea and lens. The $1 / \mathrm{e}^{2}$ laser-spot diameter formed on the retina (Fig. 5.3) by chromatic dispersion was determined by superimposing refractive error measurements of human eyes onto a simple rhesus eye model (see section 2.2.3) (Vincelette, Rockwell et al. 2008). The predicted diameter formed at the rhesus retina for laser-radiation wavelengths of 1110 , 1130 and $1150 \mathrm{~nm}$ is $146.5,150.6$, and $153.8 \mu \mathrm{m}$ respectively. The variability between these three wavelengths' laser-spot diameter due to chromatic dispersion is less than $5 \%$. In Fig. 5.3, the laser-spot diameter for the 1319-nm wavelength is approximately 184.6 $\mu \mathrm{m}$, or approximately $25 \%$ larger than the other three laser-radiation wavelengths in this study.

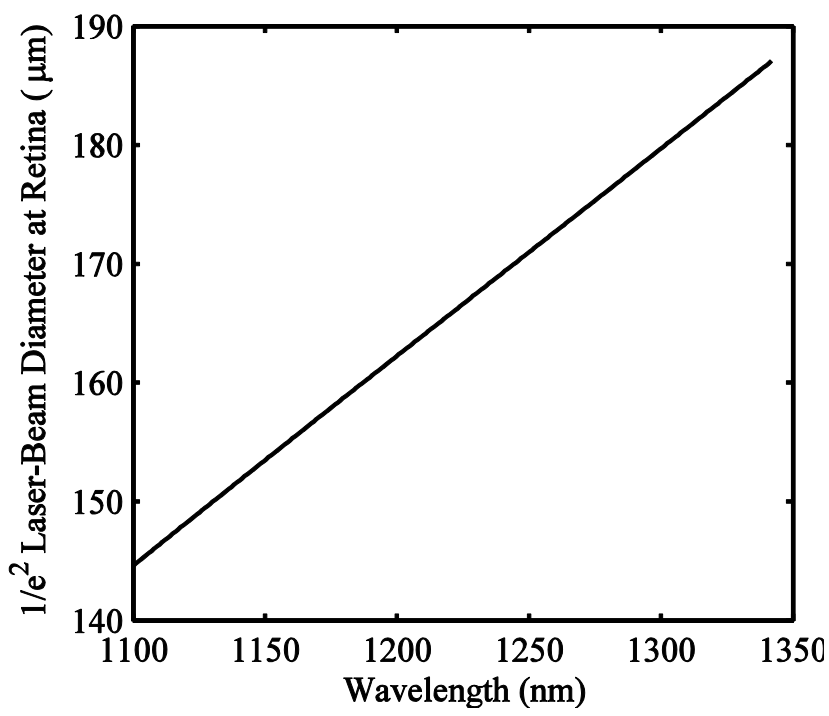

Figure 5.3: The 1/ $\mathrm{e}^{2}$ laser-spot diameter for NIR laser radiation wavelengths delivered to the rhesus retina with chromatic dispersion.

Of the three fundamental criteria leading to retinal damage from $\mathrm{CW}$ laser radiation presented in Fig.'s 5.1-5.3, the transmitted energy is by far the distinguishing variable between 1110, 1130 and 1150-nm wavelengths. For these three wavelengths, the absorption of the RPE and the laser-spot diameter delivered to the retina vary by less 
than 1 and 5\% respectively. However, the 1319-nm laser radiation wavelength is considerably different from the 1110,1130 and 1150-nm wavelengths with respect to each of the three fundamental criteria.

\subsection{MATERIALS AND METHODS}

\subsubsection{Experimental Set-Up}

The subjects in this study were four adult, male rhesus (Macaca mulatta), approximately 4-5 years in age. Animals used in this study were procured, maintained and used in accordance with the Animal Welfare Act and the "Guide for the Care and Use of Laboratory Animals" prepared by the Institute of Laboratory Animal Resources, National Research Council; and the ARVO Resolution on the Use of Animals in Research. Subjects were screened for any ocular defects prior to beginning the study. Subjects were chemically restrained with intramuscular injections of Telazol $(5-10 \mathrm{mg} / \mathrm{kg}$ body mass). Subjects then received a subcutaneous injection of $0.16 \mathrm{mg}$ atropine sulfate and eye drops; two drops each of proparacaine hydrochloride , $0.5 \%$, phenylephrine hydrochloride, $2.5 \%$ and tropicamide, $1 \%$, to dilate the eye. Bilateral saphenous catheters were used both for administering anesthetic (Propoflo ${ }^{\circledR} 1500 \mathrm{Mcg} / \mathrm{kg}$ bolus, then continuous rate infusion at $300 \mathrm{Mcg} / \mathrm{kg} / \mathrm{min}$ ) and warm intravenous fluids (lactated ringers $10 \mathrm{ml} / \mathrm{kg} / \mathrm{hr}$ ) throughout the procedure. Immediately after anesthesia was administered, the subject was intubated to assure the airway remained unobstructed throughout the experiment. Heart rate, respiration and oxygen saturation were monitored using a pulse oximeter. The body temperature of the subject was checked periodically and maintained with a Bair Hugger ${ }^{\circledR}$ (warm air circulating blanket). To minimize eye movements, a peribulbar injection of 1 cc Xylocaine, 4\%, was administered just before 
beginning the laser radiation exposures. The eye was held open by a wire lid speculum and irrigated with $0.9 \%$ saline solution every few seconds throughout the procedure. Subjects were securely restrained in the prone position on a custom animal stage designed to allow for careful movements of an intubated subject in front of a fundus camera (Fig. 5.4).

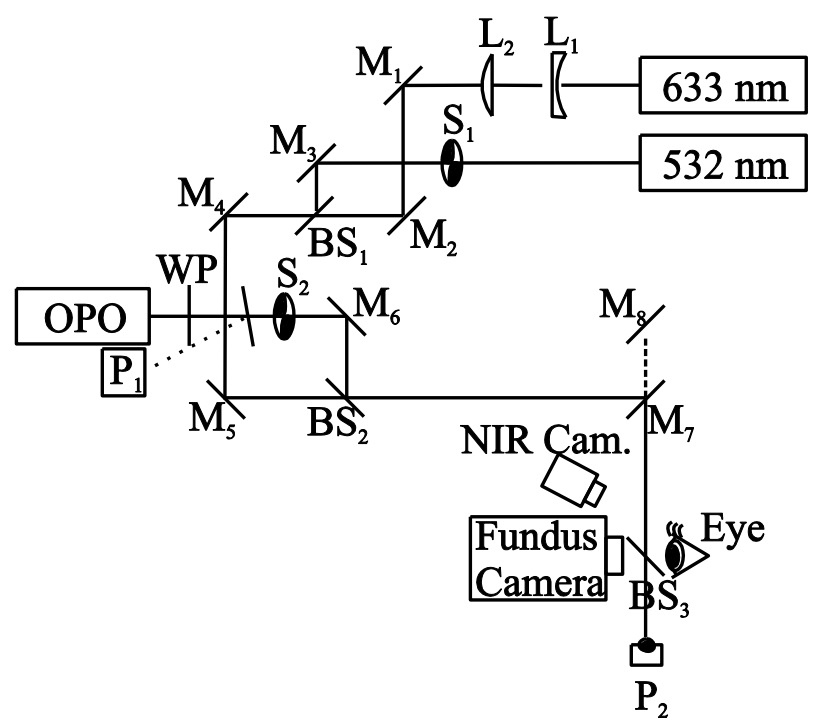

Figure 5.4: The optical set up. L1, L2 - lenses formed a 5x beam expander for the 633nm pointing laser. M1-5 - broad-band visible mirrors. M6-8 - silver mirrors. M7 and M8 formed a periscope to bring the beam up vertically to the viewing level of the fundus camera. BS1 - a beam splitter which combined the two visible lasers. BS2 and BS3identical beam splitters used to combine all three wavelengths. WP - waveplate to control power level sent down the optical train. S1 and S2 were two independently controllable electronic shutters set for exposure durations of $\sim 25$ and $100 \mathrm{~ms}$ respectively. P1 - reference power meter to determine the power to be delivered to the corneal plane. P2 - photodiode connected to an oscilloscope to verify the exposure duration of the laser-radiation. NIR Cam. - near-infrared camera used to ensure no clipping of the pupil was occurring during laser exposure.

A Coherent Chameleon laser set to 834-nm wavelength pumped the Coherent Mira-OPO tuneable laser used for the 1110, 1130 and 1150-nm wavelengths. A Lee laser was used for the 1319-nm wavelength. See Table 5.2 for a data-matrix with parameters of exposures. 
Table 5.2: The parameter sets for CW NIR exposures investigated in this study. P and $\mathrm{M}$ are for paramacula and macula respectively. A Mira-OPO laser was used for the 1110, 1130 and $1150-\mathrm{nm}$ wavelength $(\lambda)$ studies while a Lee laser was used for the 1319-nm study. The OPO and Lee laser sources had a $\theta$, full-angle beam divergence, of 1.5 and $13.3 \mathrm{mrad}$ respectively.

\begin{tabular}{|c|c|c|c|}
\hline$\lambda(\mathrm{nm})$ & $\begin{array}{c}\text { Exposure } \\
\text { Duration } \\
(\mathrm{ms})\end{array}$ & $\begin{array}{c}1 / \mathrm{e}^{2} \text { Diameter } \\
\text { at Corneal } \\
\text { Plane (mm) }\end{array}$ & $\begin{array}{c}\text { Exposure } \\
\text { Location }\end{array}$ \\
\hline 1110 & 100 & 4.8 & $\mathbb{P}$ \\
1130 & 100 & 4.8 & $\mathbb{P}$ \\
1150 & 100 & 4.8 & $\mathbb{P}$ \\
1319 & 80 & 5.0 & $\mathbf{M}$ \\
\hline
\end{tabular}

The bandwidth of the OPO laser ranged between 12 and $15 \mathrm{~nm}$ full-width at half max. A waveplate and an electronic shutter were used to control the power and exposure duration of the NIR laser at the corneal plane. A thin piece of glass placed slightly off axis just before the shutter was used to reflect a small portion of the NIR laser radiation to a Coherent power meter, PM3, read by a Coherent PowerMax 5000; this is referred to as the reference power meter. The OPO was confirmed to lase in $\mathrm{TEM}_{00}$ mode with its Gaussian beam having a full-angle divergence and $1 / \mathrm{e}^{2}$ diameter at the corneal plane of 1.5-mrad and $4.8-\mathrm{mm}$ respectively, within the specifications by the manufacturer. A Melles Griot 633-nm HeNe laser was expanded and attenuated for use as a pointer to allow for careful placement of retinal lesions. A Spectra Physics 532-nm Millennia laser was used to place marker lesions to map a grid on the retina. A second electronic shutter controlled the delivery of the 532-nm laser radiation to the ocular plane. All three laserwavelengths (NIR, 633 and 532-nm wavelengths) were co-aligned to deliver laser radiation to the ocular plane of the subject. The subject was placed such that its fundus was in focus and observable by a fundus camera by the scientific team (Fig. 5.4) with a 1in beam splitter placed just between the fundus camera's lens and the subject's eye. This beam splitter was highly transmissive in the visible allowing for a clear fundus image 
even during laser exposure. The total length of the optical train from the OPO laser head to the corneal plane was measured to be $261 \mathrm{~cm}$.

With the OPO tuned to the desired wavelength, the alignment of the system was checked 24-hours and 30-minutes before the arrival of the subject. At these times, another Coherent power meter was placed at the corneal plane and the ratio of the reference power meter to the delivered corneal power was recorded. The ratio of the 532$\mathrm{nm}$ laser radiation delivered to the corneal plane compared to the laser head setting was also obtained. These ratios were to compute the power delivered to the corneal plane and to ensure stability and repeatability of the experiments. To verify the proper exposure duration, a photodiode connected to an oscilloscope was placed perpendicular to the corneal plane. In addition to the photodiode, an Electro Physics NIR camera was placed off axis to zoom in on the corneal plane and recorded on a DVD. The NIR camera helped to confirm there was no clipping of the subject's iris when delivering the NIR laser radiation. The fundus camera was equipped with a digital camera mounted to view the fundus in addition to a $35-\mathrm{mm}$ film camera mounted in another position to take traditional photos. Fundus photographs were taken pre, 1-hr and 24-hr post NIR laser radiation exposure.

A set of 532-nm marker lesions using $48 \mathrm{~mW}$ at the corneal plane exposed for 20 ms, were made along the macula-paramacula boundary to map out a grid for referencing laser exposures in our data set. NIR laser radiation, all 100-ms, exposures were then placed along the grid map in the paramacula. Subject's maculas were not available to use in the study using the OPO laser. The paramacula grid location and reference power meter reading were recorded. Three observers inspected the subject's retina for lesions at 1-hr and 24-hr end points. These "lesion/no lesion" data were processed in Probit 
analysis software to determine the estimated dose which causes damage $50 \%$ of the time, called an $\mathrm{ED}_{50}$. Slit lamp photographs were taken to visually inspect for damage to the cornea and lens post-exposure. To check for any leakage from the placed lesions, fundus fluorescein angiography, FFA, was performed and recorded using 35-mm film immediately following the 1 and 24-hr retinal lesion reads. The dose levels for FFA were 0.5-1 $\mathrm{mg} / \mathrm{kg}$ IV (acepromazine) given $20 \mathrm{~min}$ prior to administering the Fluorescein $(0.4 \mathrm{ml} /$ subject $)$ dye.

The exposures for the 1319-nm laser radiation used a similar experimental technique, save for the laser source was a LEE-laser and the shutter was manufactured by nmLaser Products Inc. Exposures at 1319-nm were for 80-ms to the macula of the same subjects used in the OPO experiment. The 1319-nm beam characteristics at the corneal

plane were a $1 / \mathrm{e}^{2}$ diameter of $\sim 5 \mathrm{~mm}$ with a full-angle beam divergence of $13.3 \mathrm{mrad}$ and $\mathrm{m}^{2}$ of $\sim 50$.

During the course of collecting images from FFA, it was discovered that the fundus camera's filter had deteriorated, making for poor quality images taken of FFA for the $1110,1130,1150$ and some of the $1319-\mathrm{nm}$ subjects. The filter was replaced late in the 1319-nm wavelength study.

\subsubsection{Creation of the Scaling Factor}

An estimated fit of the damage threshold data was performed using a scaling algorithm developed with the three fundamental criteria previously described in Chapter 2 (Lund and Edsall 1999; Lund, Edsall et al. 2000; Lund, Edsall et al. 2005; Lund, Edsall et al. 2008; Vincelette, Rockwell et al. 2009). The scaling factor (SF) was created by breaking the fundamental criteria into a simple heat source term, $\mathrm{S}\left[\mathrm{W} / \mathrm{cm}^{3}\right]$, for a wavelength, $\lambda$, in the NIR based on 


$$
S(\lambda)=\frac{P}{\pi r^{2}}\left(\mu_{a}\right)
$$

where $\mathrm{P}$ is the power reaching the retina in $\mathrm{W}, \mathrm{r}$ is the $1 / \mathrm{e}^{2}$ radius of the beam at the retina (Fig. 5.3) in cm, and $\mu_{\mathrm{a}}$ is the linear absorption coefficients (assumed to include water) of $\mathrm{RPE}$ in $\mathrm{cm}^{-1}$ respectively (Fig. 5.2). If an input is assumed to be unity at the cornea, then the energy reaching the retina can be taken as the fraction of transmitted power, $\mathrm{P}_{\mathrm{T}}$, to the retina based on the results from Fig. 5.1.

The simple heat source term, S, can be calculated for the NIR region using Eq. 5.2, then divided by $\mathrm{S}(1110-\mathrm{nm})$ to create the scaling factor, SF, relative to the $1110-\mathrm{nm}$ damage threshold. Thus, the SF for the NIR is calculated by

$$
S F=\frac{\left(P_{T, 1110-n m} / r_{1110-n m}^{2}\right)}{\left(P_{T, \lambda} / r_{\lambda}^{2}\right)}\left(\frac{\mu_{a, 1110-n m}}{\mu_{a, \lambda}}\right) .
$$

A fit to $\mathrm{ED}_{50}$ data in the NIR can then be made by multiplying the SF by the experimental 1110-nm ED $\mathrm{E}_{50}$ value.

\subsection{RESULTS}

Probit analysis of the yes/no data at 1-Hr and 24-hr for each wavelength exposure for individual and grouped subjects is in Table 5.3. Probit analysis is the method of choice for analyzing lesion/no lesion data preferred by the Air Force Research Laboratory, Optical Radiation Branch (Cain and Noojin 1996). Probit results for combined subjects in Table 5.3 represent the complete Probit analysis performed on all the yes/no data obtained from the corresponding data set at the reported laser-radiation wavelength. Probit analysis should not be confused as an average over all subjects. Slit lamp photography always appeared negative for any laser effects in the cornea, aqueous 
and lens. The Gaussian near-infrared lasers had $1 / \mathrm{e}^{2}$ diameters of $4.8 \mathrm{~mm}$ and 5 for the OPO and LEE lasers, at the cornea, respectively. The OPO and LEE lasers' full-angle beam-divergence were 1.5 and $13.3 \mathrm{mrad}$ respectively.

Table 5.3: Summary of the data obtained from the retinal ( $\mathrm{P}$ for paramacula, $\mathrm{M}$ for macula) damage thresholds. The slopes of all Probit results were greater than 2, save for the $1150-\mathrm{nm}$ radiation cases for subjects 2, 3 and combined 1,2 \& 3 where N/A is entered. N/A entered for an $\mathrm{ED}_{50}$ indicates no lesions were observed, even though exposures were at $\sim 830-\mathrm{mW}$ (maximum power available for $1150-\mathrm{nm}$ ) for $100-\mathrm{ms}$. N/A entered in a fiducial limit (FL) indicates the FL was unattainable for the relevant data set.

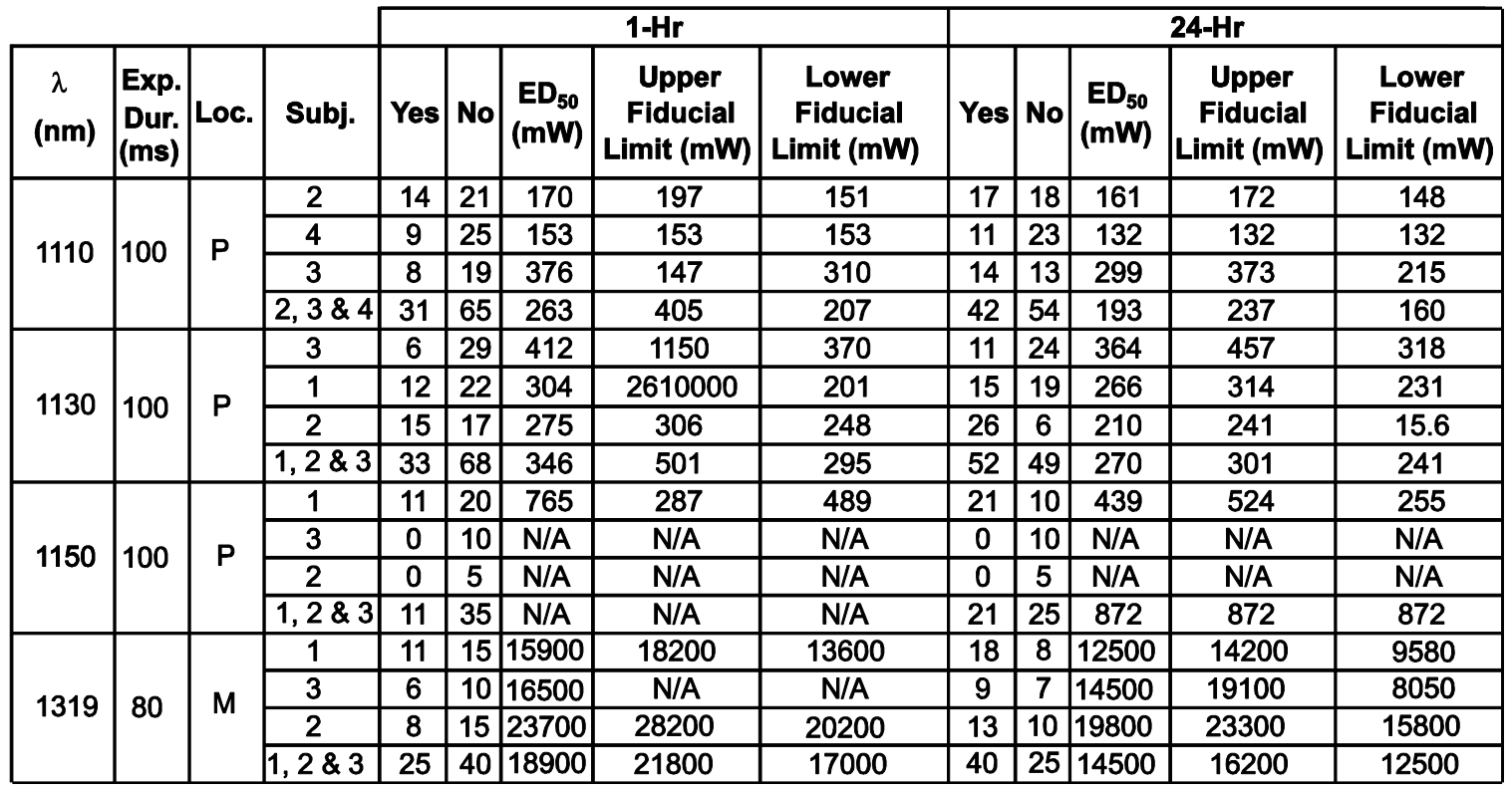

The 80-ms exposure durations for the 1319-nm laser radiation wavelength can be scaled to 100-ms exposures for comparison by (Henderson and Schulmeister 2004) (note - the $\mathrm{ED}_{50}$ 's must be converted to units of Joules to use Eq. 5.4)

$$
\mathrm{ED}_{50-100 \mathrm{~ms}}=\mathrm{ED}_{50-80 \mathrm{~ms}} *(100 / 80)^{(-1 / 4)} \text {. }
$$

From Eq. 5.2, the scaling factor, SF, for a variety of wavelengths is given in Table 5.4 . 
Table 5.4: The scaling factor, SF, from Eq. 5.2, for several wavelengths in the NIR. The $\mathrm{SF}$ is intended to be multiplied by the $1110-\mathrm{nm}$ laser radiation wavelength's $24-\mathrm{Hr} \mathrm{ED}_{50}$.

\begin{tabular}{|c|l|}
\hline$\lambda(\mathrm{nm})$ & \multicolumn{1}{|c|}{$\mathrm{SF}$} \\
\hline 1100 & 0.872 \\
1110 & 1.000 \\
1130 & 1.563 \\
1150 & 6.119 \\
1200 & 12.299 \\
1250 & 12.343 \\
1300 & 27.899 \\
1315 & 60.267 \\
1319 & 79.696 \\
1320 & 85.898 \\
\hline
\end{tabular}

The 24-hr ED $\mathrm{ED}_{50}$ data in Table 5.3 are plotted in Fig. 5.5 (all 1319-nm data were scaled to 100 -ms using Eq. 5.4). A fit to the $\mathrm{ED}_{50}$ data in Fig. 5.5 was made by multiplying the SF (Table 5.4), by the 24-hr $\mathrm{ED}_{50}$ value for combined subjects at 1110 $\mathrm{nm}$ laser radiation (193-mW delivered to cornea).

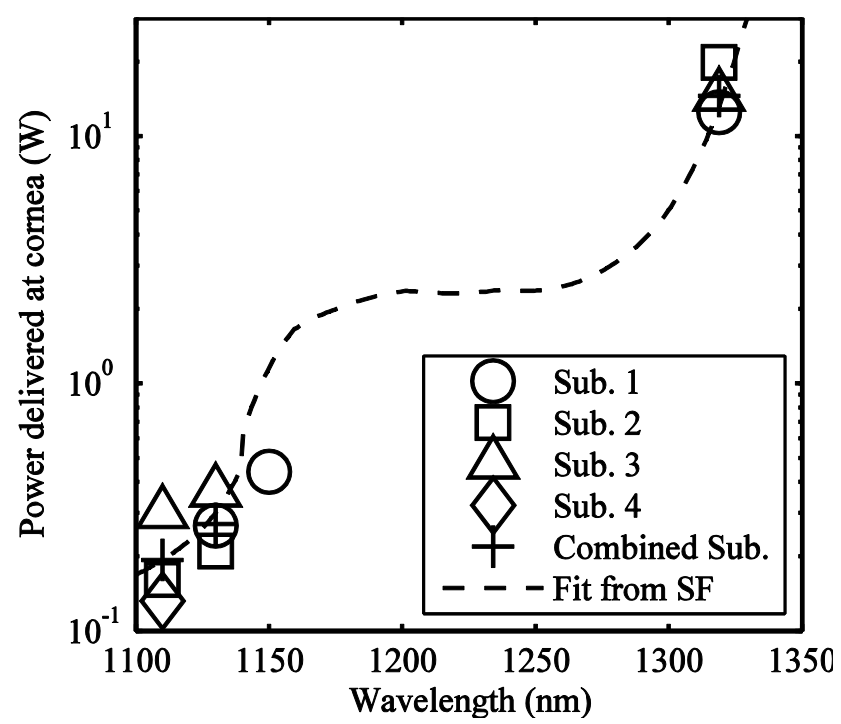

Figure 5.5: The 24-Hr, 100-ms, $\mathrm{ED}_{50}$ retinal damage thresholds from Table 5.3 (1319$\mathrm{nm}$ data were scaled to $100-\mathrm{ms}$ by Eq. 5.4) and the SF fit determined by mulitplying the SF values (Table 5.4) by the $1110-\mathrm{nm} \mathrm{ED}_{50}$ threshold for combined subjects. 
Subject 1 was exposed to $1150-\mathrm{nm}$ laser radiation in the paramacula on two separate occasions; 3-weeks apart. The first set of exposures resulted in very large lesions (large at 1-Hr, and even larger at 24-Hr) with an $\mathrm{ED}_{50}$ of 406-mW, see Fig. 5.6a, while the second set of exposures resulted in much smaller lesions (remained small at 1 and 24-Hr) with an $\mathrm{ED}_{50}$ of $567 \mathrm{~mW}$, see Fig. 5.6b. All exposure data from this subject for this wavelength were used in the Probit analysis to give the overall $\mathrm{ED}_{50}$ of $439 \mathrm{~mW}$ to obtain the value reported in Table 5.3 for subject 1 at $1150-\mathrm{nm}$.

(a)

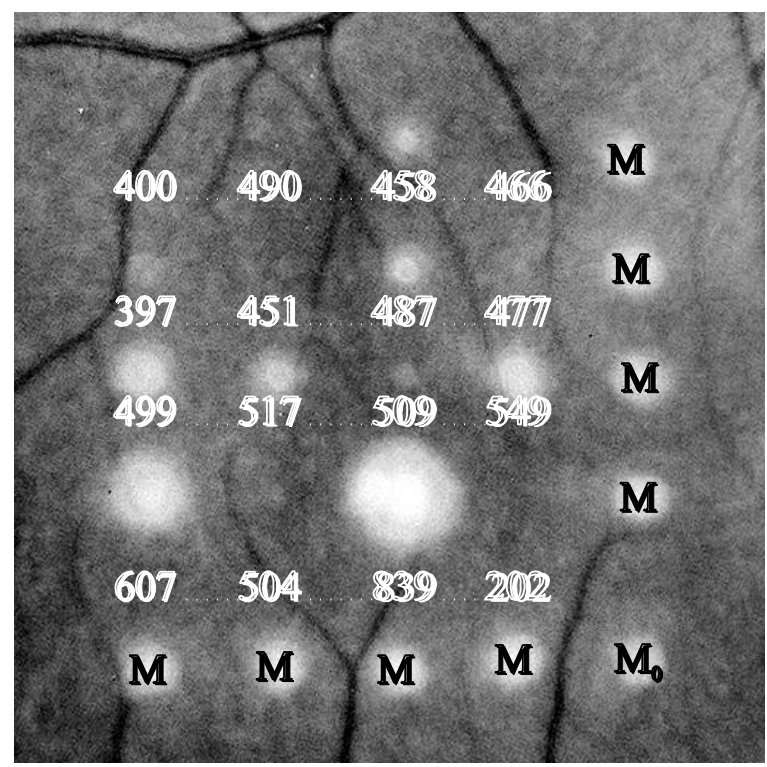

(b)

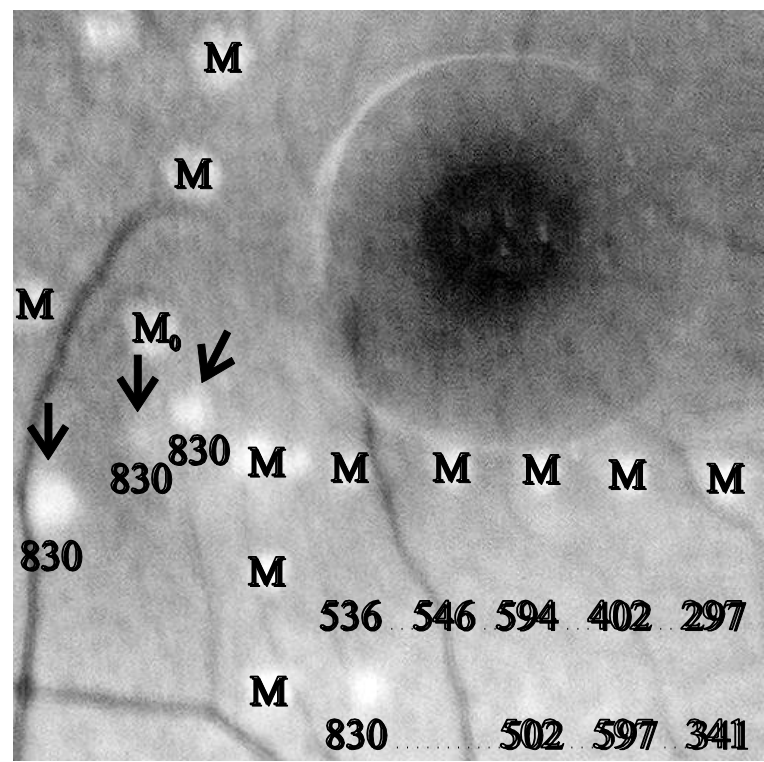

Figure 5.6: Fundus camera images captured of subject 1 at 24-Hr post-exposure to 1150$\mathrm{nm}$ laser radiation (field of view was $35^{\circ}$ for both images). The letter $\mathrm{M}$ denotes a marker lesion, with $\mathrm{M}_{0}$ acting as a reference marker between $\mathrm{a}$ and $\mathrm{b}$. The numbers marked below the grid locations represent the power, in $\mathrm{mW}$, delivered to the corneal plane for 100-ms. Lesions without numbers are marker lesions used to reference the grid location of each NIR radiation exposure on the fundus. (a) Displays the large lesions observed for the first 1150-nm exposure with a resulting $\mathrm{ED}_{50}$ of $406 \mathrm{~mW}$. (b) Displays lesions observed for the second 1150-nm exposure, conducted 3-weeks later, on the same eye resulting in an $\mathrm{ED}_{50}$ of $567 \mathrm{~mW}$. The arrows point to exposure areas off the marker grid in attempts to check experimental repeatability. Combining all yes/no data for both exposure sessions (data from both a and b) gave the resulting $\mathrm{ED}_{50}$ of $439 \mathrm{~mW}$ reported in Table 5.3. 


\subsection{DISCUSSION}

The way in which lesions are detected determines the damage endpoint. In this study, areas which were known to have been exposed to a preset dose of near-infrared, NIR, laser radiation were deemed positive for a lesion if the area appeared different from the baseline observation prior to exposure. Lesions were based only on fundus camera observation. The lesions in this study appeared as highly reflective spots on the retina. All NIR retinal lesions typically appeared larger than the marker lesions produced by the 532-nm wavelength laser. At higher NIR power exposures, lesions commonly appeared larger than lesions from lower power exposures (Fig. 5.6a). Lesions which were observed at 1-hr appeared to grow over the 24-hour period while many lesions only became apparent at the 24-hr endpoint (Table 5.3). These observations are similar to those made by Zuclich, Lund et al. 2004, and Zuclich, Lund et al. 2007 who also reported that the increased reflectivity is associated with inflammation in the retina. The growth of the reflective area is believed to be due to delayed onset inflammation; a characteristic of NIR laser-tissue interaction with the retina, believed to be related to the strong increase in water absorption at these laser-radiation wavelengths. This would result in a more uniformly distributed heating effect in the retina compared to a localized effect due to strong RPE absorption at visible laser radiation wavelengths. In the study for this chapter, power levels that produced lesions never resulted in a retinal hemorrhage.

In regards to the $100-\mathrm{ms}$ paramacular exposures to the 1150 -nm laser radiation, only one subject out of three tested, yielded any funduscopically observable lesions. This single subject's $1-\mathrm{Hr} \mathrm{ED}_{50}$ data were not statistically significant since the upper fiducial limit was greater than $50 \%$ of the corresponding $\mathrm{ED}_{50}$. However, the 24-Hr Probit of subject 1's 1150-nm wavelength data set maintained the upper and lower fiducial limits 
within $50 \%$ of the $\mathrm{ED}_{50}$. Subject 1's $24-\mathrm{Hr} \mathrm{ED}_{50} 1150$-nm laser radiation threshold value of $439 \mathrm{~mW}$ (delivered to the cornea) was a power level of approximately 2.27 times greater than the 193-mW 24-Hr $\mathrm{ED}_{50}$ from 1110-nm. Based upon Fig. 5.5, the 100-ms $\mathrm{ED}_{50}$ for $1150-\mathrm{nm}$ laser radiation is $1181 \mathrm{~mW}$, or a SF of $\sim 6$ times higher than the 1110$\mathrm{nm} 24-\mathrm{Hr} \mathrm{ED}_{50}$ (Table 5.4), but the OPO laser was only capable of delivering up to $\sim 870$ $\mathrm{mW}$, to the corneal plane. Subjects 2 and 3 were tested at the 1150 -nm laser radiation wavelength and yielded no funduscopically observable lesions on the retina. The $24-\mathrm{Hr}$ $\mathrm{ED}_{50}$ values followed the SF trend well for subjects 2 and 3 at 1110, 1130 and 1319-nm laser radiation wavelengths and for Subject 1 at 1130 and 1319-nm.

The SF method previously presented based on the three fundamental criteria creates a useful technique for fitting $\mathrm{ED}_{50}$ data to 100-ms exposures from NIR laser radiation. The SF method predicted the 1319-nm laser radiation $\mathrm{ED}_{50}$ for combined subjects to be $15.3-\mathrm{W}$ delivered at the cornea. This predicted value is only $\sim 12 \%$ larger than the $24-\mathrm{Hr} \mathrm{ED}_{50}$ experimental value of $13.7-\mathrm{W}$ in Table 5.3 (experimental value was scaled to the 100-ms exposure using Eq. 5.4). This error is within the upper and lower fiducial limits of the 24- $\mathrm{Hr} \mathrm{ED}_{50}, 15.3$ and 11.8-W respectively (fiducial limits here were scaled to 100-ms exposures using Eq. 5.4), from the experiment (Table 5.3). The action spectrum produced by Lund, Edsall et al. 2008, shows that the expected $\mathrm{ED}_{50}$ for $100-\mathrm{ms}$, $1110-\mathrm{nm}$ laser radiation is approximately a little less than $200-\mathrm{mW}$ power delivered to the cornea. Our experimental data from 3 subjects for 100-ms, 1110-nm laser radiation yielded an $\mathrm{ED}_{50}$ of $193-\mathrm{mW}$ power at the corneal plane, very close to the prediction made by Lund, Edsall et al. 2008. The SF values presented here are based on the 1110-nm reference of 193-mW delivered to the cornea. It should be stressed that the SF algorithm depends on relative values between wavelengths for percent transmission, absorption and 
beam-waist radii delivered to the retina and does not require absolute values. More advanced modeling methods are required to determine and understand absolute values and their relationship on retinal damage thresholds.

For the 1150-nm wavelength, subject 1 was exposed to this laser radiation early on in the study, and brought in a second time three weeks later to determine if the 1150$\mathrm{nm}$ lesions were repeatable. In the first 1150-nm exposure, at 1-Hr, subject 1 had large lesions even at 500-mW delivered to the cornea and an 1150-nm 24-Hr $\mathrm{ED}_{50}$ (from this first exposure set only) of $407 \mathrm{~mW}$ (see Fig. 5.6a). In the second 1150-nm exposure, the only $1-\mathrm{Hr}$ lesions were the $830-\mathrm{mW}$ power levels delivered to the cornea giving much smaller visible lesions than the first data set with a resulting 1150-nm 24-Hr ED ${ }_{50}$ (from this second exposure set only) of $567 \mathrm{~mW}$ (see Fig. 5.6b). The pigmentation of the two regions in the paramacula which were exposed to the 1150-nm laser radiation were noted to be fairly uniform and did not appear any lighter or darker than any other subject's paramacula.

The SF method predicts the $\mathrm{ED}_{50}$ of 1150 -nm laser radiation (100-ms exposure) should be $\sim 6$ times greater (Table 5.4) than the 1110-nm ED F0 $_{\text {(Fig. 5.5). Subject } 1}$ yielded 1130 and 1319-nm $\mathrm{ED}_{50}$ results consistent with the SF method (Table 5.3 and Fig. 5.5), suggesting absorptive properties of this subject's retina are not largely deviant from other subjects. Given these data trends and the fact that Subject 1 had two very different $\mathrm{ED}_{50}$ 's over a 3-week interval for the 1150 -nm exposure with remarkably smaller lesions on the second exposure, it is possible that subject 1's eye may have changed over the course of several weeks in between exposures. If the SF value is taken to be the first exposure set for subject 1 at the 24-hr read giving 406-mW 1150-nm ED 50 divided by the $193-\mathrm{mW} 1110-\mathrm{nm} \mathrm{ED}_{50}$, it then becomes a factor of 2.1 as opposed to the 
expected SF value of 6.1. Using $\mathrm{SF}=2.1$ and letting all other parameters remain the same (respective to wavelength), Eq. 5.3 can be used to solve for what $1 / \mathrm{e}^{2}$ beam-waist radius for the 1150-nm wavelength would be required to give this lower than expected SF value. Solving this, the beam-waist radius is determined to be $45.1-\mu \mathrm{m}$, compared to the expected 76.9- $\mu \mathrm{m}$ radius based on chromatic dispersion. All subject's were checked for refractive error several months prior to beginning the damage threshold studies, but are not routinely checked. In addition, pathlength is never measured at any point in the study. Perhaps the subject 1 changed over the 3-week period or a muscle, due to an imbalance in drug delivery, was pulling on the eye causing an unusual, and unpredictable, optical path in the eye. Another possibility is in the water content of subject 1's ocular tissues, though this variation would have to be fairly extreme to cause such a drastic change in $\mathrm{ED}_{50}$ thresholds.

\subsection{CONCLUSION}

The experimental $\mathrm{ED}_{50}$ retinal thresholds for 100-ms exposures to laser radiation wavelengths at 1110, 1130 and 1319-nm were determined to be 193, 270 and 13713-mW of power delivered to the corneal plane respectively. The experimental $\mathrm{ED}_{50}$ retinal threshold for $100-\mathrm{ms}$ exposures to laser radiation at the $1150-\mathrm{nm}$ wavelength was not achieved due to limitations on the availability of power from the laser source. The scaling factor, SF, method allows for trends in the fundamental criteria of transmitted energy, retinal absorption and refractive error to shape the fit, as opposed to exact values of these criteria which are difficult to determine. Given that the $\mathrm{ED}_{50}$ for 1150 -nm laser radiation was achieved on one subject, it may be worthwhile to routinely screen for refractive error and measure the ocular pathlength of subjects prior to beginning a NIR 
retinal threshold experiment. This would eliminate the uncertainty in the subject's pathlength or refractive error variability whether from growth or anesthesia.

If the 100-ms $\mathrm{ED}_{50}$ thresholds for 1110, 1130, 1150 and 1318-nm laser radiation wavelengths are used to calculate the refractive error induced in the eye by thermal lensing from the results in Chapter 3, specifically in Fig. 3.11a, then the refractive shift due to thermal lensing can be approximated as $0.22,0.44,1.12$, and $5.89 \mathrm{D}$ at the end of a 100-ms exposure for each wavelength respectively (note - the refractive shift values reported here were tabulated by scaling the input power needed for delivery to the Caincell artificial eye to achieve an equivalent fluence at the retina in the longer rhesus eye). The $100-\mathrm{ms} \mathrm{ED}_{50}$ thresholds were determined using $1 / \mathrm{e}^{2}$ beam diameters of 4.8 to $5-\mathrm{mm}$ delivered to the cornea, where-as, in Chapter 3 , the $1 / \mathrm{e}^{2}$ beam diameter delivered to the surface of the Cain-cell lens was 3.95-mm. In Chapter 4 analysis of the thermal lensing model indicates that the strength of the thermal lens induced in the eye is related to the beam-diameter delivered to the corneal plane; where the strength of the thermal lens increases with decreasing beam-diameter at the cornea (Vincelette, Thomas et al. 2009). The strength of the thermal lens for $1 / \mathrm{e}^{2}$ beam-diameters of $\sim 4-5-\mathrm{mm}$ delivered to the cornea is approximately the same, though a beam-diameter of $3.95-\mathrm{mm}$ will thermally lens only slightly more than a $4.8-\mathrm{mm}$ diameter (based on Fig. 4.8). The results from Chapter 3 are believed to provide a good approximation on the amount of thermal lensing induced in the eye. Thus, the amount of thermal lensing is greatest for the 1318-nm wavelength due to the fact more power is required to yield a lesion at the retina compared to the other wavelengths. However, the SF method does not consider thermal lensing, yet predicts the $\mathrm{ED}_{50}$ damage threshold for $1319-\mathrm{nm}$ very well. Thermal lensing is occurring in ocular media; it is a natural phenomenon, but there appears to be a disconnect in the 
way in which we have understood the creation of near-infrared laser lesions on the retina if a $5 \mathrm{D}$ shift at $1318-\mathrm{nm}$ is not circumventing retinal damage. This leads to the content of Chapter 6 which uses the first-order thermal lensing model to explore when thermal lensing may and may not be a significant laser-tissue interaction in the processes leading to retinal damage.

\subsection{ACKNOWLEDGEMENTS}

I would like to thank the following people for their assistance with the data collection and/or analysis presented in this chapter:

Ms. Aurora Shingledecker, Mr. Kurt Schuster, Mr. Gary Noojin, and Mr. Dave Stolarski, from Northrop Grumman

Dr. Ben Rockwell, Dr. Jeff Oliver and Dr. Semih Kumru, from the Air Force Research Laboratory, Brooks City-Base 


\section{CHAPTER 6}

\section{FIRST-ORDER THERMAL LENSING MODELING PREDICTIONS OF RETINAL DAMAGE THRESHOLD}

\subsection{ABSTRACT}

The retinal damage threshold study from Chapter 5 suggested the action spectrum based upon changes in the spot-size delivered to the retina using chromatic dispersion, neglecting thermal lensing, was sufficient to make retinal damage predictions for large beam-diameters ( $\sim 5-\mathrm{mm})$ delivered to the cornea for wavelengths between 1100-1319nm. In this study, data from Chapter 5, for 1110, 1130, 1150, 1310 and 1319-nm wavelengths were used as input parameters for the first-order thermal lensing model, presented in Chapter 4, to generate values for $\mathrm{dT} / \mathrm{dt}$ then integrated over time to predict the temperature rise at the retina for $\mathrm{CW}$ exposure durations. The temperature rise at the retina was calculated for a series of input beam-diameters and powers delivered to the corneal plane. The predicted damage threshold for each parameter set was then determined by calculating when the retinal temperature reached a selected threshold temperature damage condition. Analysis included comparing the predicted retinal damage threshold with and without thermal lensing. Using equivalent conditions, the first-order model was also compared to results from the BTEC model reported by Clark, Irvin et al. 2008 with and without axial conduction. The first-order modeling results predicted thermal lensing begins to inhibit retinal damage for wavelengths above 1300$\mathrm{nm}$ at higher power and smaller beam-diameters delivered to the corneal plane. Results are intended to be used as a guide for when thermal lensing becomes a significant lasertissue interaction in continuous-wave near-infrared laser radiation exposures to the retina. 


\subsection{INTRODUCTION}

Early in vivo retinal threshold studies using a continuous-wave (CW) 1318-nm laser source performed by Zuclich, Lund et al. 2004 were unable to produce retinal lesions in the conventional rhesus animal model. However, recent retinal damage threshold studies from CW near-infrared, NIR, laser radiation at wavelengths greater than 1100-nm have been determined (Chapter 5).

The scaling factor (SF) method, presented in Chapter 5, based on the action spectrum created by Lund, Edsall et al. 2008 was found to fit the 50\% estimated damage threshold $\left(\mathrm{ED}_{50}\right)$ data at 1110,1130 , and 1319-nm wavelengths within $10 \%$ of the experimental values. The SF method algorithm did not include time dependent processes such as thermal lensing. The fact that these data follow predicted time-independent trends suggested thermal lensing, a time-dependent process, did not afford protection of the retina from CW NIR laser radiation.

The first-order thermal lensing model was found to be suitable for analyzing trends of thermal lensing in a simple-eye model (Chapter 4) and predicted that thermal lensing was strongly related to the beam-diameter delivered to the corneal plane such that thermal lensing increases with decreasing corneal beam-diameter. For the $\mathrm{ED}_{50}$ threshold case of the 1319-nm wavelength with $14.5-\mathrm{W}$ of power and a $5-\mathrm{mm}$ beam diameter delivered to the corneal plane, the first-order model predicted the beam-waist radius at the retina would only change by no more than a factor of $\sim 2.25$ at the end of the $80-\mathrm{ms}$ exposure. It is possible that for a large beam-diameter delivered to the cornea, similar to those used in experimental retinal $\mathrm{ED}_{50}$ studies, the role of thermal lensing is minimized in the eye such that it afforded no protection to the retina. The CW NIR laser radiation damage threshold for beam-diameters, delivered to the cornea, smaller than 4.8 to $5 \mathrm{~mm}$, 
have yet to be tested in an animal model. Experiments to address these findings are being planned by the Air Force Research Lab.

In the first portion of this chapter, the temperature rise at the retina was estimated using the first-order model of thermal lensing in a simple eye (Chapter 4). Assuming a peak temperature for damage based on recent experiments at the Air Force Research Lab, $\mu_{\mathrm{a}}$ for the retinal pigment epithelium (PE) was computed given experimentally determined threshold powers: (data from Chapter 5) selected wavelengths were 1110, 1130, 1150, 1310 and 1319-nm. The input beam-diameter and/or power delivered to the cornea were then varied in the model, for comparison to the selected threshold temperature, and the exposure time needed to achieve a retinal lesion was computed. Data from this segment of the study are intended for use as an aid to predict under which conditions the thermal lens becomes a significant factor in the occlusion of retinal damage from CW near-infrared laser radiation.

Limitations of the first-order thermal lensing model were the absence of axial conduction and the retina and PE were modeled as a single layer. In the second portion of this chapter, the 1319-nm laser radiation wavelength was processed in the first-order thermal lensing model for a homogeneous, water-filled eye. The simulation was run for a set of input parameters for large and small beam diameters and at a high and low power for comparison to the Air Force Research Laboratory's BTEC model results with and without axial thermal conduction (Clark, Irvin et al. 2008). Data from this portion of the study were used to validate the temperature calculation implemented in the first-order thermal lensing model. 


\subsection{BACKGROUND}

The eye is a very complex optical system, but was reduced to a simple system for the first-order model (see Fig. 6.1). The simple eye used in this study was treated as a series of index-matched slabs with all the focusing power being performed by the singlecurved surface at the air-cornea boundary. The thermal and optical components of the model eye for each wavelength under investigation are presented in Table 6.1. The retina is also a very complex tissue comprised of many function-specific cells stratified into multiple layers. The retinal pigment epithelium (RPE) layer, found approximately 150 $\mu \mathrm{m}$ into the retina, contains pigment granules which are believed to be the primary absorber of laser radiation reaching the retina. Pigmented granules are present in both the RPE and choroid tissue behind the retina as reported in a histological study on the Macaca mulatta (common name - rhesus monkey) by Birngruber, Hillenkamp et al. 1985. Birngruber, Hillenkamp et al. 1985 stated that the choroid of the rhesus monkey has a homogenous pigmentation at the avascular areas similar to the pigment epithelium layer and is approximately $80-\mu \mathrm{m}$ thick. The area known as the choriocapillaris, approximately $20-\mu \mathrm{m}$, is a pigment-free layer between the RPE and the choroid. Given these histological findings, the absorption coefficients for PE and choroid can be approximated as being equal (Birngruber, Hillenkamp et al. 1985).

The first-order model presented in Chapter 4 includes a final step in the algorithm to compute the change in temperature, $\Delta \mathrm{T}$, for a given axial position in terms of the time dependent $1 / \mathrm{e}^{2}$ beam-waist radii reaching the retina with radial thermal diffusion. This is achieved by integrating 


$$
\left.\left(\frac{\partial T}{\partial t}\right)\right|_{r=0}=\left.\eta\left(2 \frac{\partial^{2} T}{\partial r^{2}}+\frac{1}{\kappa} S(z)\right)\right|_{r=0}
$$

over time, t. The term $\eta$ is the thermal diffusivity, $\kappa$ is the thermal conductivity, $z$ is the optical axis, and $\mathrm{r}$ is the radius perpendicular to $\mathrm{z}$. Notice this integration is evaluated for $\mathrm{r}=0$. The source term, $\mathrm{S}(\mathrm{z})$, was defined in Eq. 4.15 and $\partial^{2} \mathrm{~T} / \partial \mathrm{r}^{2}$ is defined in Eq. 4.17 from the first-order model presented in Chapter 4.

Typically, tissue damage calculations are based upon the Arrhenius integral, which assumes an isothermal event, given as

$$
\Omega(r, z)=A \int_{0}^{\infty} \exp \left(\frac{-E_{a}}{R T(r, z, t)}\right) d t
$$

where $E_{a}$ is the activation energy required to denature the tissue, $R$ is the universal gas constant, A is the molecular collision frequency factor and $\Omega$ is the damage parameter (Pearce and Thomsen 1995; Gerstman and Glickman 1999; Pearce and Thomsen 2003; Lund 2006; Chen 2007; Thomas, Vincelette et al. 2007; Clark, Irvin et al. 2008). The exact values for $E_{a}$ and $A$ are difficult to determine and the solution assumes the process is isothermal. A compilation of values have been reported for $\mathrm{A}$ and $\mathrm{E}_{\mathrm{a}}$ for the retina, ranging from $4.3 * 10^{64}$ to $3.1 * 10^{99} \mathrm{~s}^{-1}$ and $2.93 * 10^{5}$ to $6.7 * 10^{5} \mathrm{~J} /$ mole respectively (Pearce and Thomsen 1995). However, CW exposures to the retina are non-isothermal, which has recently lead into an investigation on cell death using microthermography funded by the Air Force (Denton, Foltz et al. 2009).

Using an in vitro retinal model, Denton, Foltz et al. 2009 recorded the temperature profiles of $\mathrm{PE}$ cells exposed to various 514-nm CW laser radiation exposures as a function of time. Damage to the cells was believed to be predominantly thermal in nature for the laser radiation exposures. Temperature profile data were overlayed with 
fluorescently labeled images to correlate with cell death to peak and average temperatures. Data revealed that cells in the in vitro experiment consistently died when an average peak temperature was achieved regardless if the exposure duration was 0.1 , 0.25 or $1.0 \mathrm{~s}$. This average peak temperature was determined by the temperature averaged over the rim, or perimeter, of dead cells. Denton, Foltz et al. 2009 suggested that a PE cell will die if the laser exposure causes the cell to reach the average peak temperature, $\mathrm{T}$, threshold of just below $\sim 50^{\circ} \mathrm{C}$. Furthermore, preliminary examination of their 0.1 and 0.25 -s data for damage rate processes did not follow the Arrhenius model. Denton, Foltz et al. 2009 are currently submitting these data for publication in Nature Methods. Other investigations into thermal damage in tissue from $\mathrm{CW}$ laser radiation have reported a temperature threshold relationship between acute damage and cell survival for laser exposures to osteocytes and prostate cells (Sugita, Osa et al. 2009; van Nimwegen, L'Eplattenier et al. 2009).

Pearce and Thomsen 1995, Fig. 17.17, reported damage processes in terms of temperature and exposure duration for skin, egg white and collagen. These data reported an estimated absolute temperature between $\sim 23$ and $\sim 50^{\circ} \mathrm{C}$ for 100 -ms exposures. Pearce and Thomsen 1995 data were for tissues and not cell cultures as in the Denton, Foltz et al. 2009 report. In facing the difficulty with selecting a damage temperature condition for the modeled data in this chapter, a bound was placed on the data such that the Denton, Foltz et al. $2009 \Delta \mathrm{T}$ of $13^{\circ} \mathrm{C}$ and the Pearce and Thomsen $1995 \Delta \mathrm{T}$ of $50^{\circ} \mathrm{C}$ represent the lower and upper limits of the bounds respectively. It is not unreasonable to select the Denton, Foltz et al. 2009 data for a lower bound since their damage end point was observable cell death for an in vitro model. For in vivo retinal lesion studies, the damage end point was taken to be the lesions which were observable by funduscopic observation 
which leaves undeterminable factors for this end point; the limit of resolution of the fundus camera and the lesion observer's ability to discern lesions.

Considering the cells constituting the neural retina are free of pigment granules, the retinal tissues anterior to the RPE layer, but are believed to be involved with the formation of retinal lesions, these cells must have an absorption coefficient similar to that of water. If the RPE is more highly absorbing than water, then the proximity of the neural retina layer means conduction from the RPE layer increases the peak average temperature in the neural retina. Given the results from Denton, Foltz et al. 2009 and the complex nature in selecting rate coefficients for the Arrhenius equation (Eq. 6.2), the damage end-point used in this chapter was taken to be when the $\mathrm{T}(\mathrm{r}=0, \mathrm{z}=$ anterior retinal surface, $\mathrm{t}_{0}$ ) calculated for a specific wavelength, input beam diameter and power was equal to the calculated $\mathrm{T}_{0}\left(\mathrm{r}=0, \mathrm{z}=\right.$ anterior retinal surface, $\left.\mathrm{t}_{0}\right)$ for a referenced input beam diameter and power which caused experimental threshold damage (see Chapter 5) ( $\mathrm{t}_{0}=80 \mathrm{~ms}$ for $1319-\mathrm{nm}$ and $100-\mathrm{ms}$ for all other wavelengths). This analysis assumed that the peak temperature rise at the retina was achieved at the end of the exposure for the experimentally determined input parameters allowing for $\mu_{\mathrm{a}}$ to be selected based upon wavelength. The selected $\mu_{\mathrm{a}}$ represented an average $\mu_{\mathrm{a}}$ for the retina assuming direct absorption of the PE layer plus water absorption which gave rise to the threshold peak temperature, with a discrete $\mu_{\mathrm{a}}$ for each lower and upper bound of $\Delta \mathrm{T}$ at each wavelength.

Since the assumption that the temperature rise is the result of direct heating of the PE plus water absorption is made and the values for $\mu_{\mathrm{a}}$ for each wavelength used for the retina were selected based upon the upper and lower bounds of the peak threshold temperature, it should be understood that the value of $\mu_{\mathrm{a}}$ for the retina has been simplified in this approach. The exact optical absorption of the neural retina, RPE, and choroid 
tissues are not known in the NIR wavelength region. These assumptions were made to simplify a complex issue such that thermal lensing could be compared within a single wavelength data set, where retinal absorption would be the same. This enabled data to be examined by when the thermal lens would begin to alter $\mathrm{T}(\mathrm{t})$ achieved at the retina significantly. The fact that a $\Delta \mathrm{T}_{0}$ was used to establish damage in this study also approximated the relative proportionality of the $\mu_{\mathrm{a}}$ between wavelengths (i.e. ratio between $\mu_{\mathrm{a}}$ 1110-nm: $\mu_{\mathrm{a}}$ 1319-nm, etc.) for the retina and PE which was found to be an important role in fitting retinal $\mathrm{ED}_{50}$ data in Chapter 5 (Eq. 5.3). This means $\mu_{\mathrm{a}}$ determined for the lower bound of $\Delta \mathrm{T}$ should approximately maintain proportionalities for all lower $\Delta \mathrm{T}$ data, and $\mu_{\mathrm{a}}$ determined for the upper bound $\Delta \mathrm{T}$ data should follow suit for all respective upper bound data. Note that Chapter 5 used values of $\mu_{\mathrm{a}}(\lambda)$ based upon a fit to data from Birngruber, Gabel et al. 1985 which resulted in unreasonably high peak $\Delta \mathrm{T}$ values on the order of $\sim 400^{\circ} \mathrm{C}$ as determined by the algorithm from the first-order themal lensing model in this study.

Both the first-order themal lensing and BTEC models assumed the retina had the same thermal and optical properties as the vitreous. The simulations stopped at the anterior surface of the retinal layer and neglected axial conduction. More advanced beam propagation models, like those at the Air Force Research Lab, will use a more advanced model where the neural-retina $(\sim 150-\mu \mathrm{m}$ pathlength) and PE (5- $\mu \mathrm{m}$ pathlength) are treated as separate layers and scattering effects on beam propagation can be incorporated into the model. Data for the ocular chamber pathlengths for the rhesus monkey are from Fernandes, Bradley et al. 2003. The refractive index of the simple eye model as a function of wavelength was reported previously in Chapter 2 in section 2.2.3 (Vincelette, 
Rockwell et al. 2008). The thermal and optical properties of each media can be found in Tables 6.1 and 6.2 respectively.

Figure 6.1: The simple eye model for a rhesus subject. The radius of curvature, $r_{c}$, on the cornea is $0.498 \mathrm{~cm}$, in order to achieve a minimum beam-waist for a collimated beam of 589-nm laser radiation at the front surface of the retina. The front-surface of the retina layer was placed along the $\mathrm{z}$-axis at $1.99 \mathrm{~cm}$ after the corneal surface.

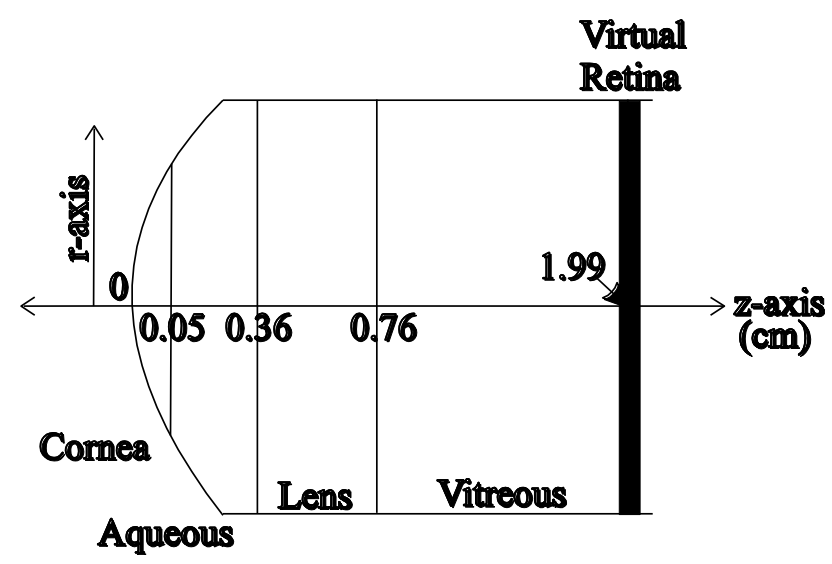

Table 6.1: The thermal coefficients of the simple rhesus eye needed for the thermal lensing model. The terms $\kappa, \rho$ and $c_{p}$ are the thermal conductivity, density and specific heat at constant pressure respectively. Values for the cornea, aqueous, lens and vitreous were obtained from Okonu, Kojima et al. 2005, and values for water were from Wagner and Kruse 1998. All parts of the retina were assumed to have the same thermal properties as the vitreous.

\begin{tabular}{|c|c|c|c|}
\cline { 2 - 4 } \multicolumn{1}{c|}{} & $\begin{array}{c}\kappa \\
{[\mathrm{W} / \mathrm{mK}]}\end{array}$ & $\begin{array}{c}\rho \\
{\left[\mathrm{kg} / \mathrm{m}^{3}\right]}\end{array}$ & $\begin{array}{c}\mathrm{c}_{\mathrm{p}} \\
{[\mathrm{J} / \mathrm{kgK}]}\end{array}$ \\
\hline Cornea & 0.500 & 1050 & 3470 \\
\hline Aqueous & 0.623 & 994 & 4180 \\
\hline Lens & 0.430 & 1080 & 3470 \\
\hline Vitreous & 0.623 & 994 & 4180 \\
\hline Retina & 0.623 & 994 & 4180 \\
\hline Water & 0.623 & 994 & 4180 \\
\hline
\end{tabular}


Table 6.2: The optical coefficients of the simple rhesus eye model. The terms $\mathrm{n}_{0}$ and $\mu_{\mathrm{a}}$ are the background index of refraction (all layers of the eye are treated as index matched) and the linear absorption coefficient respectively. The values for $\mathrm{n}_{0}$ were determined previously in Chapter 2 and Vincelette, Rockwell et al. 2008. The values for $\mu_{\mathrm{a}}$ were from Maher 1978, Hale and Querry 1973, and by fitting a curve to data from Birngruber, Hillenkamp et al. 1985. (C - cornea, A - aqueous, L - lens, V - vitreous, $\mathrm{W}$ - water, PE - pigment epithelium). Note the PE values reported here were not used for this study; they are presented merely for comparison and assumed to include water.

\begin{tabular}{|c|c|c|c|c|c|c|c|}
\hline \multirow{2}{*}{$\begin{array}{c}\lambda \\
{[\mathrm{nm}]}\end{array}$} & \multirow{2}{*}{$\mathrm{n}_{0}$} & \multicolumn{6}{|c|}{$\mu_{\mathrm{a}}\left[\mathrm{cm}^{-1}\right]$} \\
\cline { 3 - 8 } & & $\mathrm{C}$ & $\mathrm{A}$ & $\mathrm{L}$ & $\mathrm{V}$ & $\mathrm{W}$ & $\mathrm{PE}$ \\
\hline 1110 & 1.3187 & 0.945 & 0.103 & 0.223 & 0.316 & 0.345 & 97.900 \\
\hline 1130 & 1.3183 & 1.141 & 0.274 & 0.316 & 0.483 & 0.563 & 89.300 \\
\hline 1150 & 1.3179 & 1.511 & 1.221 & 0.607 & 1.131 & 0.777 & 81.500 \\
\hline 1310 & 1.3149 & 1.820 & 1.734 & 0.858 & 1.641 & 1.229 & 39.126 \\
\hline 1319 & 1.3148 & 2.002 & 2.048 & 0.982 & 1.940 & 1.360 & 37.500 \\
\hline
\end{tabular}

The first-order thermal lensing model, presented in Chapter 4, predicts that the strength of the thermal lens greatly increases as the beam diameter delivered to the corneal plane decreases in size (Vincelette, Thomas et al. 2009). The strength of the thermal lens (described as the ratio of the initial $1 / \mathrm{e}^{2}$ beam-waist radius at time $=0$ to the radius at time $=100 \mathrm{~ms}$ ) is given as a series of beam-diameters input into the cornea for 1110, 1150, and 1319-nm wavelengths in Table 6.3. The irradiated area at the retina does not change significantly for the larger beam-diameters according to the thermal lensing model trend. 
Table 6.3: The increase from initial beam diameter at the retinal plane for the retinal $\mathrm{ED}_{50}$ 's of 1319,1150 and 1110-nm wavelengths for varying input beam diameters at the corneal plane. These results were tabulated using the mathematical model presented in Chapter 4. The model's input parameters for power, exposure duration and wavelength were selected from the experimental results for the $\mathrm{ED}_{50}$ values from Chapter 5.

\begin{tabular}{|c|c|c|c|}
\cline { 2 - 4 } \multicolumn{1}{c|}{} & \multicolumn{3}{c|}{$\begin{array}{c}\text { Increase in Beam-Diameter at } \\
\text { Retinal Plane at End of CW } \\
\text { Exposure (multiplier) }\end{array}$} \\
\hline $\begin{array}{c}\text { 1/e Beam } \\
\text { Diameter Delivered } \\
\text { to Corneal Plane } \\
(\mathrm{mm})\end{array}$ & $\begin{array}{c}14.5-\mathrm{W}, \\
80-\mathrm{ms}, \\
1319-\mathrm{nm}\end{array}$ & $\begin{array}{c}1.178-\mathrm{W}, \\
100-\mathrm{ms}, \\
1150-\mathrm{nm}\end{array}$ & $\begin{array}{c}0.193-\mathrm{W}, \\
100-\mathrm{ms}, \\
1110-\mathrm{nm}\end{array}$ \\
\hline 5 & 2.26 & 1.22 & 1.03 \\
\hline 4 & 3.61 & 1.47 & 1.06 \\
\hline 3.5 & 4.96 & 1.72 & 1.10 \\
\hline 3 & 7.30 & 2.17 & 1.16 \\
\hline
\end{tabular}

The NIR lesions formed in the retina are believed to be caused by thermal damage which is directly related to the fluence rate produced at the retinal plane. Since the exact fluence rate at the retina is unknown, retinal damage is commonly reported as the amount of energy, J, per unit area delivered to the corneal plane. In this study, the predicted retinal damage conditions are reported as the power (in Watts) delivered to the retinal plane and was not scaled to the size of the beam at the cornea; rather data were evaluated in terms of varying the beam-size at the corneal plane. This was done due to the prediction that thermal lensing becomes increasingly more significant as the beam size is reduced at the corneal plane as illustrated in Table 6.3.

In Chapter 5, a scaling factor (SF) method was used to predict the damage endpoint for a series of wavelengths and exposure conditions. The scaling factor method was found to fit retinal $\mathrm{ED}_{50}$ data very well for wavelengths between 1100-1319-nm when referenced to the experimental continuous-wave $(\mathrm{CW})$ retinal $\mathrm{ED}_{50}$ for $1110-\mathrm{nm}$. The input parameters of beam-size and power delivered to the cornea and exposure 125 
duration are reported in Table 6.4. Since experimental ED ${ }_{50}$ 's for 1310 and 1150 -nm laser radiation wavelengths were not available, the SF method in Chapter 5 was used to establish reference conditions for these two wavelengths.

Table 6.4: The 24-hour $\mathrm{ED}_{50}$ from Chapter 5. ${ }^{1}$ Indicates set was determined experimentally. ${ }^{2}$ Indicates the set was estimated from the SF method.

\begin{tabular}{|c|c|c|c|}
\hline$\lambda(\mathrm{nm})$ & $\begin{array}{c}1 / \mathrm{e}^{2} \text { Beam } \\
\text { Diameter } \\
\text { Delivered to } \\
\text { Cornea } \\
(\mathrm{mm})\end{array}$ & $\begin{array}{c}\text { Exposure } \\
\text { Duration } \\
(\mathrm{ms})\end{array}$ & $\begin{array}{c}\text { Power } \\
\text { (mW } \\
\text { delivered } \\
\text { at cornea) }\end{array}$ \\
\hline $1110^{1}$ & 4.8 & 100 & 193 \\
\hline $1130^{1}$ & 4.8 & 100 & 270 \\
\hline $1150^{2}$ & 4.8 & 100 & 1178 \\
\hline $1310^{2}$ & 4.8 & 100 & 8483 \\
\hline $1319^{1}$ & 5.0 & 80 & 14500 \\
\hline
\end{tabular}

Given the fact the scaling factor method presented in Chapter 5 predicted the experimental retinal $\mathrm{ED}_{50}$ for the 1319-nm wavelength, the assumption that thermal lensing was not significant for large beam diameters delivered to the corneal plane was made. At first glance, this assumption may seem extreme given the results in Table 6.3 for the 1319-nm wavelength and the 5-mm diameter which grows in size by more than a factor of 2 in 80 -ms. The size of the beam-waist radius delivered to the retina at $t=0$ for wavelengths above $900-\mathrm{nm}$ were predicted using a linear fit to extrapolated data for chromatic dispersion. It is possible that the algorithm used to estimate chromatic dispersion in section 2.2.3 has accidentally accounted for a larger beam diameter at the retina for some of the wavelengths out in the NIR; particularly for longer wavelengths where the linear fit may have the greatest error as there are no data for chromatic dispersion in the eye above $900-\mathrm{nm}$. 
Assuming thermal lensing is occurring in the eye over the laser exposure, this implies the induced lens is not significant for the large beam diameters tested in the study for Chapter 5. By making this assumption, in the first portion of this study, the upper and lower bounds of $\mu_{\mathrm{a}}$ using the referenced retinal damage threshold temperature's upper and lower bounds of $50^{\circ} \mathrm{C}$ and $13^{\circ} \mathrm{C}$ for conditions with thermal lensing were determined. Since data are compared within a single wavelength data set, the absorption coefficient of the retina was the same between cases with and without thermal lensing. The required input power level was then determined for the case without thermal lensing to accommodate for the value of $\mu_{\mathrm{a}}$ used for the retina and PE at the tested wavelength such that the tested temperature threshold was reached in the allotted exposure time (an example of this process is presented in the results section). This allowed for two unique observations of the data: a - The time to reach the threshold temperatures could be compared independently between the cases evaluated with and without thermal lensing. $\mathrm{b}$ - The power setting required to reach the threshold temperature for the referenced $\mathrm{ED}_{50}$ exposure duration could be investigated as a function of input beam diameter. Both of these observations lend insight as to what conditions the thermal lens would protect the retina.

In the second portion of this study, the first-order thermal lensing model, FOTLM, was compared against temperature predictions compiled from the Air Force Research Laboratory's BTEC model, named after the four primary authors; Buffington, Thomas, Edwards and Clark (Clark, Irvin et al. 2008). The BTEC thermal model is a 1$\mathrm{D}$ and 2-D cylindrical coordinate system simulation of optical radiation and radio frequency thermal interaction with tissues. The code supports the illumination of tissues 
by sources, the temperature response from the linear absorption of optical radiation, and analysis of subsequent damage.

The BTEC model uses finite difference numerical methods for heat transfer in conjunction with an alternating-direction-implicit (ADI) finite difference method to solve the heat equation in 2-D and a Crank-Nicholson Method in 1-D. The BTEC model includes higher fidelity capabilities including terms for varying the surface boundary conditions and examining the single-rate process model of thermal injury (the Arrhenius equation). The BTEC model required values for the beam-waist radius, $\omega$, as a function of optical axis position, $\mathrm{z}$, and time, $\mathrm{t}$, be generated by the first-order thermal lensing model. The second portion of this study examined the temperature rise calculations at the retinal plane in both the first-order thermal lensing model, which assumed no axial conduction, and BTEC model, with and without axial conduction, for cases with and without thermal lensing. The eye model geometry, optical and thermal parameters were the same between models for equal comparison.

\subsection{MATERIALS AND MethodS}

\subsubsection{Selection of $\mu_{\mathrm{a}}$ and Predicting Damage Thresholds as a Function of Input Beam Diameter}

The first-order thermal lensing model created in Matlab from Chapter 4 was used to simulate a series of conditions for each wavelength using a variety of input parameters. First, the input parameter conditions given in Table 6.4 were used to generate the $1 / \mathrm{e}^{2}$ beam-waist radii at the retinal plane using the eye model from Fig. 6.1. The temperature rise at the retinal plane was calculated by integrating Eq. 6.1 over time using Matlab's fittype command. The thermal and absorption coefficients for pre-retinal media used in 
this portion of the study can be found in Tables 6.1 and 6.2. The thermal coefficients for the retina were assumed to be the same as vitreous. The optical coefficients, $\mu_{\mathrm{a}}$, for the retina at each wavelength were determined by finding the upper and lower limit for $\mu_{\mathrm{a}}$ which gave the $\Delta \mathrm{T}_{0}$ of $13^{\circ} \mathrm{C}$ and $50^{\circ} \mathrm{C}$ for the referenced input damage condition. Since modeling results were explored to test the hypotheses that 1 - thermal lensing happens and 2 - thermal lensing does not happen, there is a power which corresponded to a case with and without thermal lensing for each wavelength. The retina's $\mu_{\mathrm{a}}$ values for each threshold parameter set at each wavelength with thermal lensing are reported in the results section.

For example, the 1319-nm laser radiation wavelength study in Chapter 5 used a 5$\mathrm{mm}$ beam diameter at the corneal plane and $80-\mathrm{ms}$ exposures, yielding a $24-\mathrm{hr}$ retinal $\mathrm{ED}_{50}$ of $14.5-\mathrm{W}$. Thus, $\Delta \mathrm{T}(\mathrm{t})$ for the $1319-\mathrm{nm}$ cases were calculated using these input parameter conditions and the temperature value at $80-\mathrm{ms}$. The $\mu_{\mathrm{a}}$ used for the retina which gave a $\Delta \mathrm{T}_{0}$ of $13^{\circ} \mathrm{C}$ at the end of the 80 -ms exposure when thermal lensing on was $1.421 \mathrm{~cm}^{-1}$, while the case for $\Delta \mathrm{T}_{0}$ of $50^{\circ} \mathrm{C}$ determined a $\mu_{\mathrm{a}}$ of $5.463 \mathrm{~cm}^{-1}$ was needed. Thus, for all other simulations at 1319-nm, this respective $\mu_{\mathrm{a}}$ for the retina was used for cases with and without thermal lensing. When relevant, each case in the results section explicitly states the $\mu_{\mathrm{a}}$ used in the computation. Each simulation then examined when the damaging peak threshold temperature was achieved to determine which power setting was needed to create $\Delta \mathrm{T}_{0}$ at the end of an 80 -ms exposure with and without thermal lensing independently.

Using these $\mu_{\mathrm{a}}$ values for the retina, two specific cases for 1319-nm laser radiation for two different input beam diameters and equivalent input powers are presented in sufficient detail to juxtapose the results with and without thermal lensing from the upper 
and lower extremes on the influence of thermal lensing in this study. For each case, the computational beam-waist radius at the retina, source term, $\mathrm{dT} / \mathrm{dt}$, and temperature rise at the retina as a function of time with and without thermal lensing were graphed. These presented cases used the input parameter conditions for the experimental retinal $\mathrm{ED}_{50}$ thresholds for respective wavelengths given in Table 6.4.

The thermal lensing code was run for a series of other input parameter sets for each wavelength to determine the relationship between input beam-diameter and time to reach the $\Delta \mathrm{T}_{0}$. Data in this segment were run for both the upper and lower values of $\mu_{\mathrm{a}}$ at each wavelength. The time to reach the damage threshold temperature for each parameter set was then used to generate the predicted $\mathrm{ED}_{50}$ as a function of beamdiameter at each wavelength for comparison to the referenced parameter condition using equivalent exposure durations (80-ms for the 1319-nm wavelength and 100-ms for all other wavelengths). All simulated parameter sets are given in Table 6.5 and were run with and without thermal lensing. 
Table 6.5: The parameter sets which were simulated in this study. The temperatures of each set were calculated. The time to reach the damage threshold temperature in each set was recorded for conditions with and without thermal lensing. * - Denotes the input parameter set used to establish the damage threshold temperature for the respective wavelength.

\begin{tabular}{|c|c|c|c|}
\hline$\lambda(\mathrm{nm})$ & \multicolumn{3}{|c|}{1110} \\
\hline $\begin{array}{c}1 / \mathrm{e}^{2} \\
\text { Beam- } \\
\text { Diameter } \\
\text { at Cornea } \\
(\mathrm{mm})\end{array}$ & 4.8 & 3.5 & 3 \\
\hline $\begin{array}{c}\text { Input } \\
\text { Power at } \\
\text { Corneal }\end{array}$ & 0.100 & 0.100 & 0.100 \\
\cline { 2 - 4 } Plane (W) & 0.250 & 0.200 & 0.193 \\
\cline { 2 - 4 } & - & 0.250 & 0.250 \\
\hline
\end{tabular}

\begin{tabular}{|c|c|c|c|}
\hline$\lambda(\mathrm{nm})$ & \multicolumn{3}{|c|}{1130} \\
\hline $\begin{array}{c}1 / \mathrm{e}^{2} \\
\text { Beam- } \\
\text { Diameter } \\
\text { at Cornea } \\
(\mathrm{mm})\end{array}$ & 4.8 & 3.5 & 3 \\
\hline Input & 0.150 & 0.150 & 0.150 \\
\cline { 2 - 4 } $\begin{array}{c}\text { Power at } \\
\text { Corneal }\end{array}$ & 0.200 & 0.200 & 0.200 \\
\cline { 2 - 4 } Plane (W) & $0.270^{*}$ & 0.250 & 0.300 \\
\cline { 2 - 4 } & 0.300 & 0.300 & - \\
\hline
\end{tabular}

\begin{tabular}{|c|c|c|c|}
\hline$\lambda(\mathrm{nm})$ & \multicolumn{3}{|c|}{1150} \\
\hline $\begin{array}{c}1 / \mathrm{e}^{2} \\
\text { Beam- } \\
\text { Diameter } \\
\text { at Cornea } \\
(\mathrm{mm})\end{array}$ & 4.8 & 3.5 & 3 \\
\hline Input & 0.750 & 0.750 & 0.750 \\
\cline { 2 - 4 } $\begin{array}{c}\text { Power at } \\
\text { Corneal }\end{array}$ & $1.178^{*}$ & 0.950 & 0.900 \\
\cline { 2 - 4 } Plane (W) & 1.500 & 1.000 & 1.000 \\
\cline { 2 - 4 } & 2.000 & 1.500 & 1.500 \\
\hline
\end{tabular}

\begin{tabular}{|c|c|c|c|}
\hline$\lambda(\mathrm{nm})$ & \multicolumn{3}{|c|}{1310} \\
\hline $\begin{array}{c}1 / \mathrm{e}^{2} \\
\text { Beam- } \\
\text { Diameter } \\
\text { at Cornea } \\
(\mathrm{mm})\end{array}$ & 4.8 & 3.5 & 3 \\
\hline \multirow{2}{\text{Input}}{$\begin{array}{c}\text { Int } \\
\text { Power at } \\
\text { Corneal } \\
\text { Plane (W) }\end{array}$} & 5.000 & 5.000 & 5.000 \\
\cline { 2 - 4 } & $8.483 *$ & 8.000 & 8.000 \\
\cline { 2 - 4 } & 9.000 & 9.000 & 9.000 \\
\hline
\end{tabular}

\begin{tabular}{|c|c|c|c|c|}
\hline$\lambda(\mathrm{nm})$ & \multicolumn{4}{|c|}{1319} \\
\hline $\begin{array}{c}1 / \mathrm{e}^{2} \\
\text { Beam- } \\
\text { Diameter } \\
\text { at Cornea } \\
(\mathrm{mm})\end{array}$ & 5 & 4 & 3.5 & 3 \\
\hline \multirow{9}{*}{$\begin{array}{c}\text { Input } \\
\text { Power at } \\
\text { Corneal } \\
\text { Plane (W) }\end{array}$} & 8.00 & 8.00 & 8.00 & 8.00 \\
\hline & 10.00 & 10.00 & 10.00 & 10.00 \\
\hline & 11.00 & 12.00 & 11.20 & 14.00 \\
\hline & 12.00 & 14.00 & 12.00 & 16.00 \\
\hline & 13.00 & 16.00 & 14.00 & 18.00 \\
\hline & 14.00 & 18.00 & 16.00 & 19.50 \\
\hline & $14.50 *$ & - & 18.00 & - \\
\hline & 16.00 & \multirow{2}{*}{\multicolumn{3}{|c|}{ - }} \\
\hline & 18.00 & & & \\
\hline
\end{tabular}

\subsubsection{Comparing First-Order Model to BTEC Model Results}

The first-order thermal lensing model was run for another series of computations to populate a matrix of values for the beam-waist radius, $\omega$, over all time, $t$ and space, $z$, $\omega(\mathrm{z}, \mathrm{t})$ for $1319-\mathrm{nm}$ laser radiation for 5 and 2.5-mm input beam diameters input into a 
simulated rhesus eye. In the eye model used for this portion of the study, the absorption coefficient of the pre-retinal media was taken to be uniform and equivalent to water $\left(\mu_{\mathrm{a}}=\right.$ $1.3598 \mathrm{~cm}^{-1}$ used for cornea, aqueous, lens and vitreous). This was done merely for convenience in configuring the two models for comparison. All thermal coefficients for the ocular chambers in this portion of the study were reported in Table 6.1.

The first-order thermal lensing model was run for each of the 2.5 and $5-\mathrm{mm}$ at 0.25 and $1-\mathrm{W}$ input cases for a $1319-\mathrm{nm} \mathrm{CW}$ laser radiation source to populate a matrix of values for $\omega(\mathrm{z}, \mathrm{t})$. The first-order thermal lensing model directly computes the temperature $\mathrm{T}(\mathrm{r}=0, \mathrm{z}=1.99 \mathrm{~cm}, \mathrm{t})$, using Matlab's fittype command and Eq. 6.1. Because the BTEC model does not include the same beam-propagation method used in the first-order thermal lensing model and the objective was to compare computational temperatures for equivalent conditions, the BTEC model used a fitting algorithm to approximate the beam-radius at the retinal plane based upon the movement of the minimum beam-waist, $\omega_{\min }$, propagated in the first-order model as a function of $\mathrm{t}$ (note both models allow for chromatic dispersion such that the minimum beam-waist for a 1319-nm wavelength resides behind the retina). This required the first-order model results for $\omega_{\min }(\mathrm{z}, \mathrm{t})$ to be fed into the BTEC model. Once fed into BTEC, the BTEC model approximated the beam-waist radius at the retinal plane. Though not presented here, this approximation was found to breakdown for stronger thermal lensing cases such that the beam-waist value generated at the retinal plane was no longer comparable to the original $\omega$ from the first-order thermal lensing model. It should be understood that this error in approximating $\omega(t)$ for any other $z$ position other than the z-location of $\omega_{\min }(t)$ in the BTEC model will lead to a discrepancy between the two models. To minimize this error, weaker thermal lensing cases were used for comparing models. 
In this segment of the study, the absorption coefficient for the retina was run with $\mu_{\mathrm{a}}=1.3598 \mathrm{~cm}^{-1}$ equivalent to water. To validate the algorithm used to calculate $\Delta \mathrm{T}$ in the first-order thermal lensing model, the BTEC model was run for cases with and without axial conduction and compared to comparable simulated conditions.

\subsection{RESULTS}

\subsubsection{Predicting Damage Threshold as a Function of Input Beam Diameter Using the First-Order Thermal Lensing Model}

\subsubsection{Selection of $\mu_{a}$ for the Retina}

The temperature rise at the retina was calculated for a series of retinal $\mu_{\mathrm{a}}$ values using the input parameter sets from Table 6.4 (model geometry given in Fig. 6.1). The value of $\mu_{\mathrm{a}}$ for the retina for all remaining computations in the study were then chosen by analyzing the linear relationship between $\mu_{\mathrm{a}}$ and $\Delta \mathrm{T}_{0}$ and solving for the $\mu_{\mathrm{a}}$ which gave the approximate $\Delta \mathrm{T}_{0}$ threshold of $50^{\circ} \mathrm{C}$. These values for $\mu_{\mathrm{a}}$ for each wavelength, computed with thermal lensing, are given in Table 6.6. There is a linear relationship between $\Delta \mathrm{T}$ threshold and $\mu_{\mathrm{a}}$ unique to each wavelength (when using the input parameters in Table 6.4). 
Table 6.6: The values for $\mu_{\mathrm{a}}$ used for the homogenous retinal slab, assumed to have equal water and pigment distributed uniformly throughout, which gave the computational upper and lower limit of $\Delta \mathrm{T}\left(50^{\circ} \mathrm{C}\right.$ and $\left.13^{\circ} \mathrm{C}\right)$. All cases were computed with thermal lensing on in the first-order model. Unless stated otherwise, the $\mu_{\mathrm{a}}$ values for the upper limit were used for the remainder of the computations in this chapter. The $\mu_{\mathrm{a}}$ values for water are given for comparison and were obtained from Hale and Querry 1973. Input parameters for power and beam-diameter at each wavelength were given in Table 6.4.

\begin{tabular}{|c|c|c|c|}
\hline \multirow{2}{*}{$\lambda(\mathrm{nm})$} & \multicolumn{2}{|c|}{$\mu_{\mathrm{a}}\left(\mathrm{cm}^{-1}\right)$} & \multirow{2}{*}{\begin{tabular}{c}
$\Delta \mathrm{T}$ \\
\cline { 2 - 3 }
\end{tabular}} \\
$\begin{array}{c}\text { Threshold } \\
=13^{\circ} \mathrm{C}\end{array}$ & $\begin{array}{c}\Delta \mathrm{T} \\
\text { Threshold } \\
=50^{\circ} \mathrm{C}\end{array}$ & $\begin{array}{c}\mu_{\mathrm{a}}\left(\mathrm{cm}^{-1}\right) \\
\text { Water }\end{array}$ \\
\hline 1110 & 3.211 & 12.350 & 0.345 \\
\hline 1130 & 3.175 & 12.211 & 0.563 \\
\hline 1150 & 2.575 & 9.905 & 0.777 \\
\hline 1310 & 1.165 & 4.481 & 1.229 \\
\hline 1319 & 1.421 & 5.463 & 1.360 \\
\hline
\end{tabular}

The value of $\mu_{\mathrm{a}}$ used for the retina was tested to check if it had any influence on the time a parameter set was computed to reach the $\Delta \mathrm{T}_{0}$. This check determined that the time to achieve $\Delta \mathrm{T}_{0}$ threshold was independent of $\mu_{\mathrm{a}}$ as long as $\Delta \mathrm{T}_{0}$ was adjusted accordingly to the referenced condition.

\subsubsection{Computing T(t) Using the First-Order Thermal Lensing Model}

The cases for 1319-nm, 14.5-W, $80-\mathrm{ms}$ exposure for $1 / \mathrm{e}^{2}$ input beam-diameters of 3 and $5-\mathrm{mm}$ in the Fig.'s $6.2-6.5$ describe the step-by-step computational methods employed in this study. Using the model from Chapter 4, the first step is to generate a series of beam-waist radii at the retina as a function of time (Fig. 6.2). 
(a)

(b)

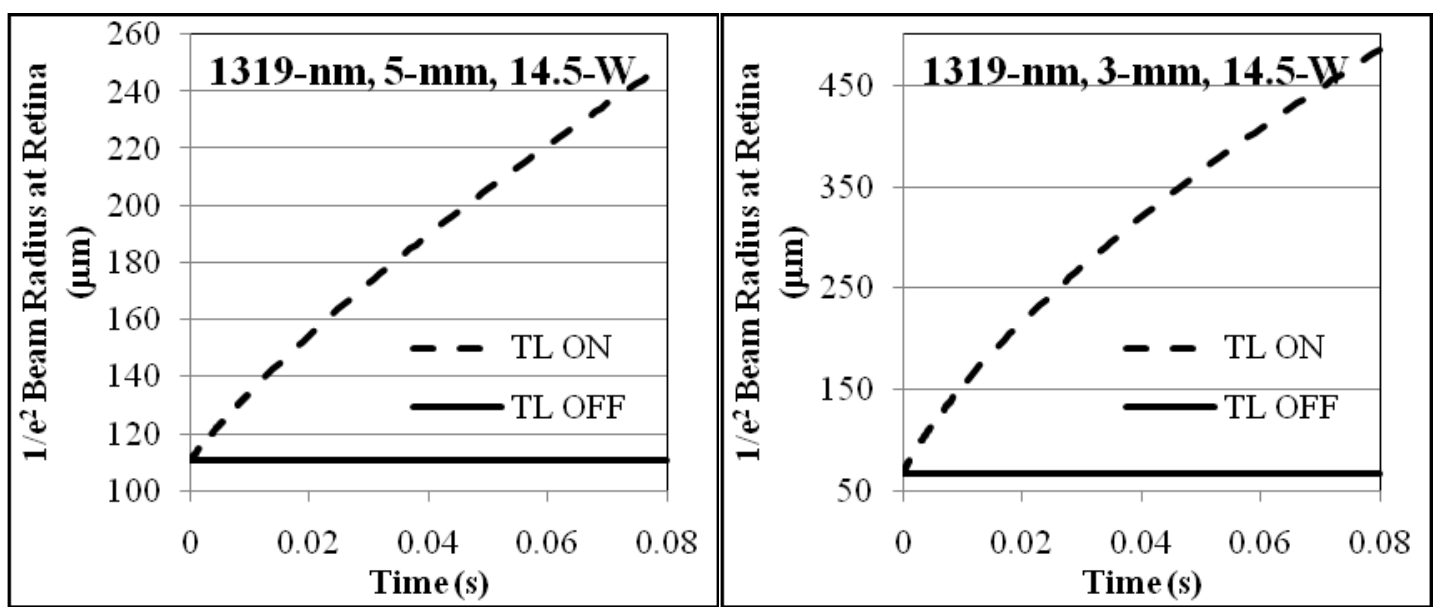

Figure 6.2: The beam-waist radius as a function of time for a collimated (a) 5-mm and (b) $3-\mathrm{mm} 1 / \mathrm{e}^{2}$ diameter delivered to the corneal plane. These data were used as inputs into the computations to determine the source term and temperature rise as the retina. (Note that this step does not require $\mu_{\mathrm{a}}$ of the retina.)

The beam-waist data in Fig. 6.2 were then used to compute the source term at the retina for cases with and without thermal lensing using the $\mu_{\mathrm{a}}$ value, respective to the 1319-nm wavelength, reported in Table 6.6 for the $\Delta \mathrm{T}_{0}$ case of $50^{\circ} \mathrm{C}$, of $5.463 \mathrm{~cm}^{-1}$. The fluence rate behaved similarly to the source term, where the term is constant over time without thermal lensing, but decreased over time with thermal lensing due to the expanding beam-waist at the retina. 
(a)

(b)

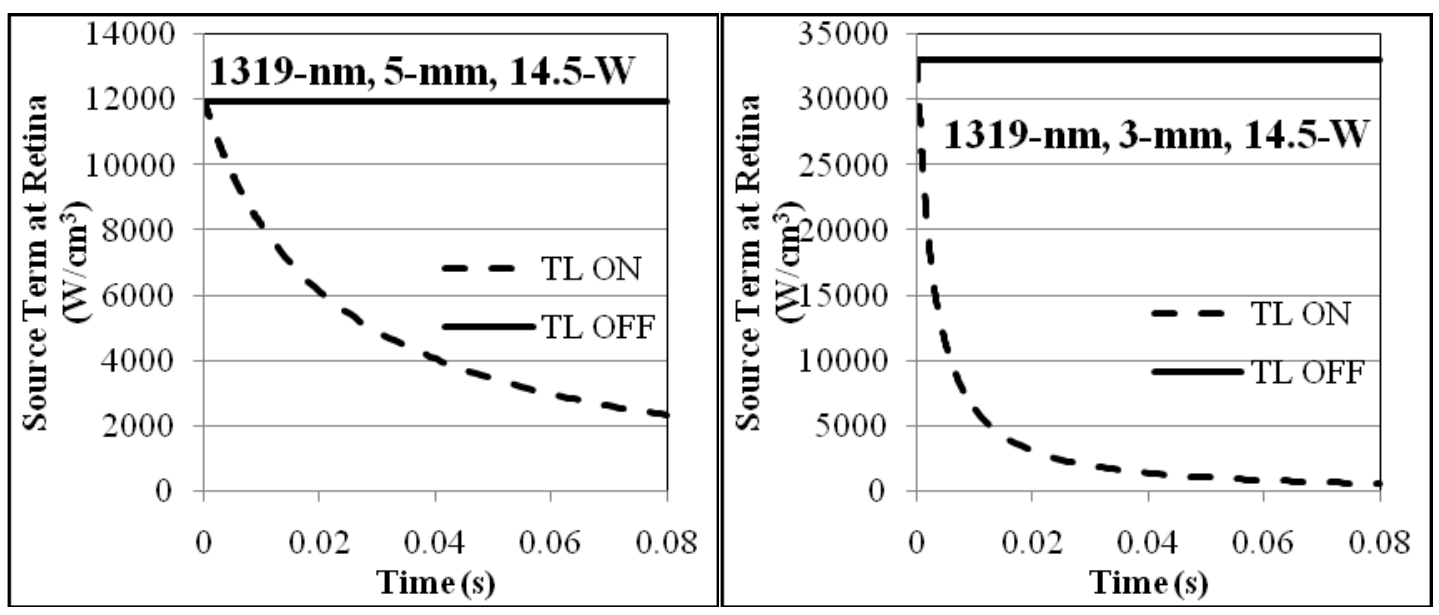

Figure 6.3: The source terms generated at the retina at $r=0$, using the $\omega(\mathrm{z}=$ retina, $\mathrm{t})$ from Fig. $6.2\left(\mu_{\mathrm{a}}\right.$ values given in Table 6.6 for $\Delta \mathrm{T}_{0}$ of $\left.50^{\circ} \mathrm{C}\right)$.

The terms for $\mathrm{d}^{2} \mathrm{~T} / \mathrm{dr}^{2}$ were then computed for each case with and without thermal lensing then combined with the source term using Eq. 4.18 which gave $\mathrm{dT} / \mathrm{dt}$ as a function of time (Fig. 6.4).

(a)

(b)

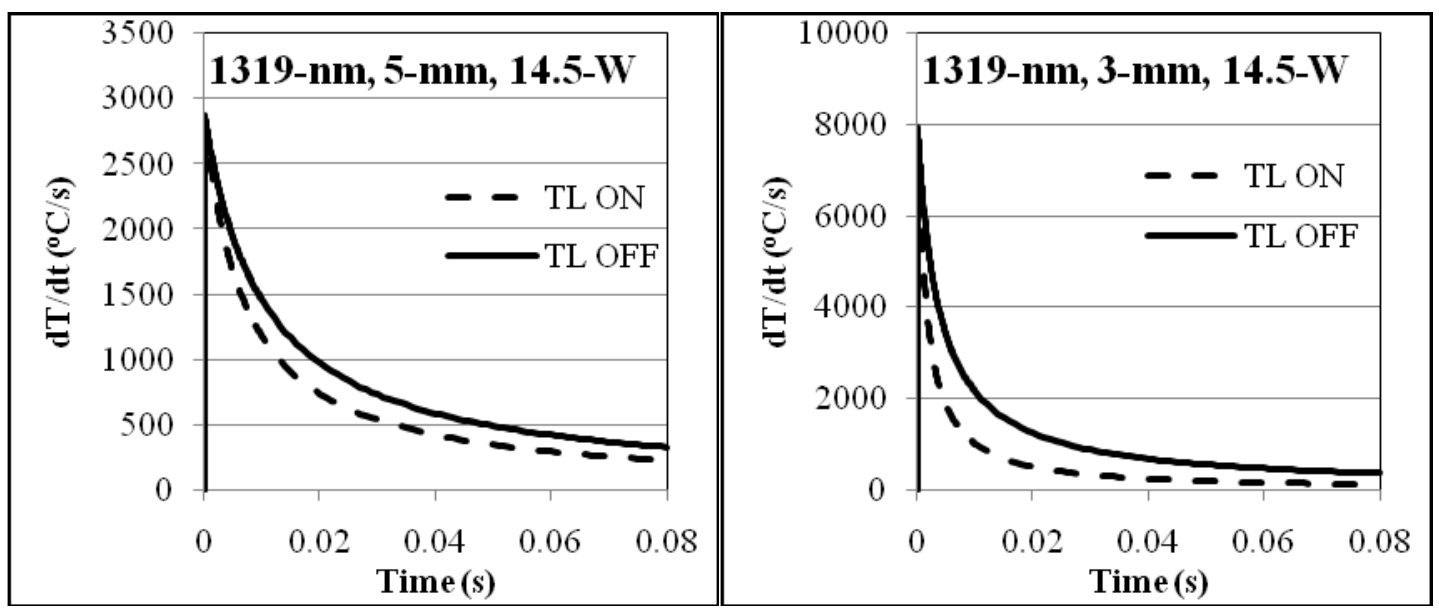

Figure 6.4: The result of Eq. 6.1 for the two specified cases with and without thermal lensing for the collimated $1 / \mathrm{e}^{2}$ input beam diameters of (a) 5-mm and (b) 3-mm. 
The results from dT/dt were then integrated over time using Matlab's fittype command to give the results in Fig. 6.5.

(a)

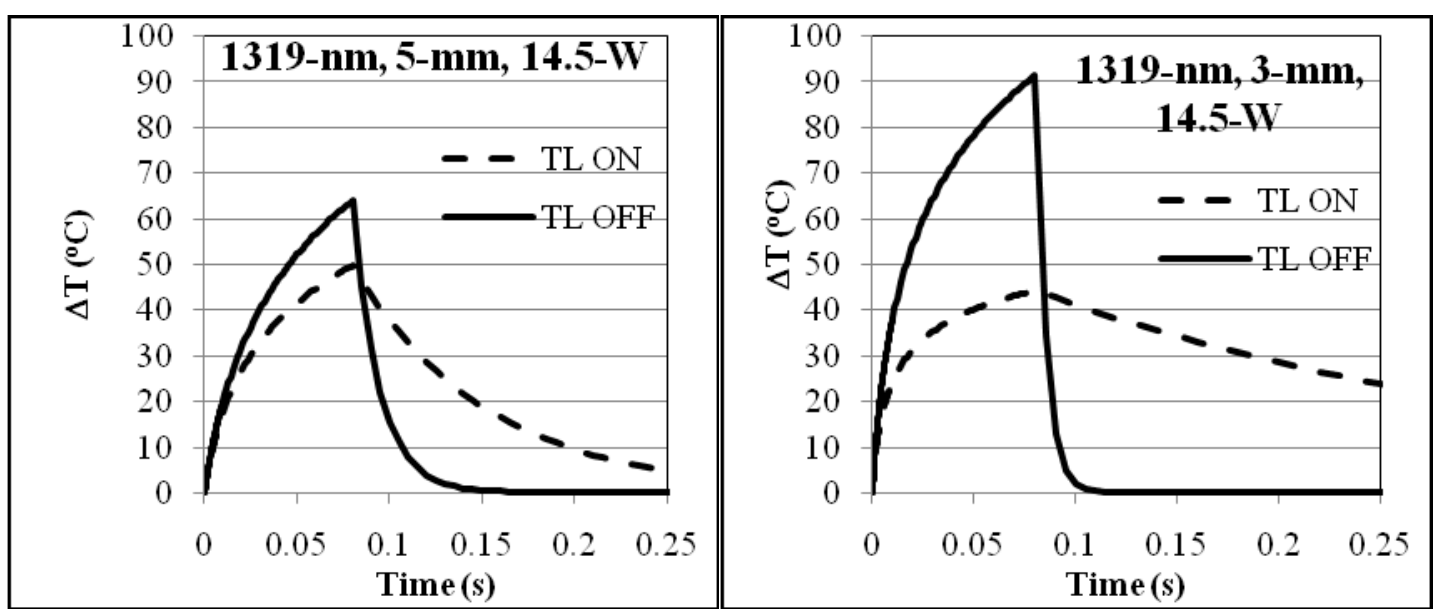

Figure 6.5: The resulting $\Delta \mathrm{T}$ at the anterior retinal surface at the $\mathrm{r}=0$ position as a function of $t$ for the two specified cases with and without thermal lensing (assuming no axial diffusion). The threshold temperature, $\Delta \mathrm{T}_{0}$, was selected to be $50^{\circ} \mathrm{C}$. The (a) $5-\mathrm{mm}$ case shows some variation in the temperature rise between the cases with and without thermal lensing. The (b) 3-mm case shows a substantial difference in the temperature rise during an 80-ms exposure.

\subsubsection{Predicted retinal damage threshold for various input beam diameter}

The simulation set given in Table 6.5 were computed then analyzed to determine the power which was needed to reach the damage threshold, $\Delta \mathrm{T}_{0}$ of $50^{\circ} \mathrm{C}$, at 80 -ms for the 1319-nm wavelength and 100-ms for all other wavelengths for each input beam-diameter at the corneal plane. All $\mu_{\mathrm{a}}$ values used for this segment were reported in Table 6.6 for the $\Delta \mathrm{T}_{0}$ condition of $50^{\circ} \mathrm{C}$. These input powers provided the estimated $\mathrm{ED}_{50}$ as a function of input beam diameter for each wavelength. It is worth reiterating the two assumptions made in regards to this approach: 1 - For the case of thermal lensing, it was assumed that there was no thermal lensing and that damage only occurred when the temperature 
reached $\Delta \mathrm{T}_{0}$. This means variations in time to reach $\Delta \mathrm{T}_{0}$ for various parameter sets are due to radial thermal diffusion at the retina, input-beam diameter and power at the corneal plane and the thermal lens induced in the ocular media. 2 - For the case without thermal lensing, it was assumed that thermal lensing was not occurring for any of the input beam-diameter conditions and that damage was reached when the power was sufficiently high enough to reach $\Delta \mathrm{T}_{0}$. This means any variation in time to reach $\Delta \mathrm{T}_{0}$ for various parameter sets was due only to radial thermal diffusion at the retina, input-beam diameter and power at the corneal plane. The results from this analysis were plotted in Matlab (Fig. 6.6) and used the absorption coefficients for the retina at each wavelength were reported in Table 6.6 for $\Delta \mathrm{T}_{0}$ of $50^{\circ} \mathrm{C}$. 
(a) 1319-nm

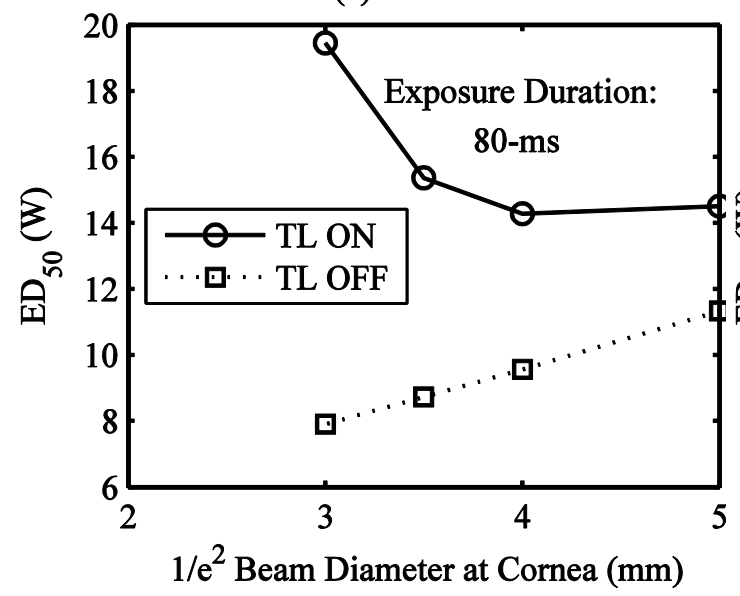

(c) $1150-\mathrm{nm}$

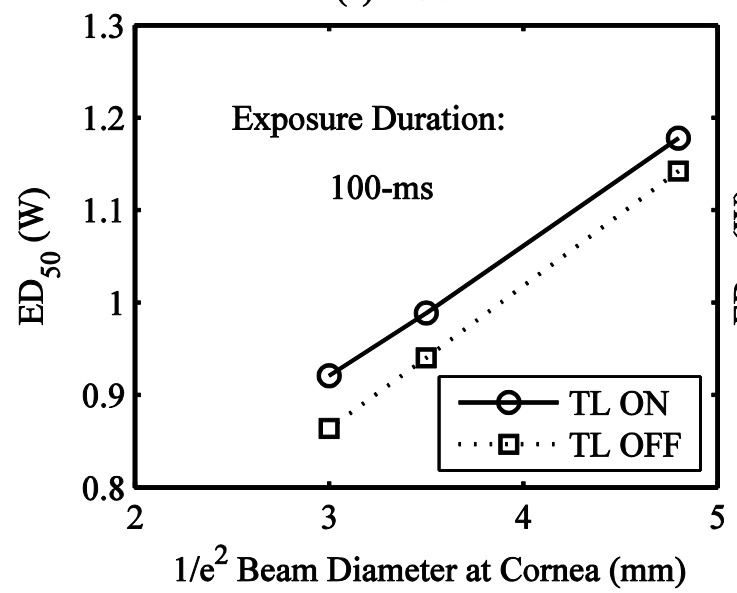

(e) $1110-n m$

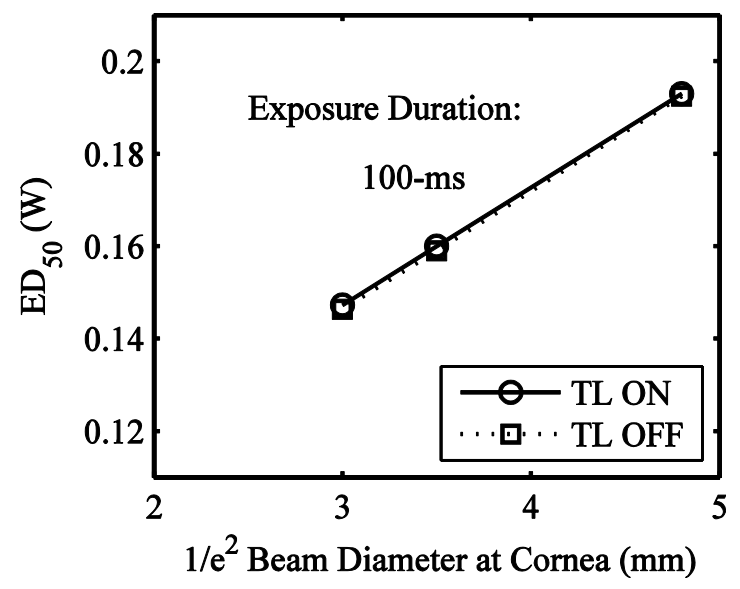

(b) 1310-nm

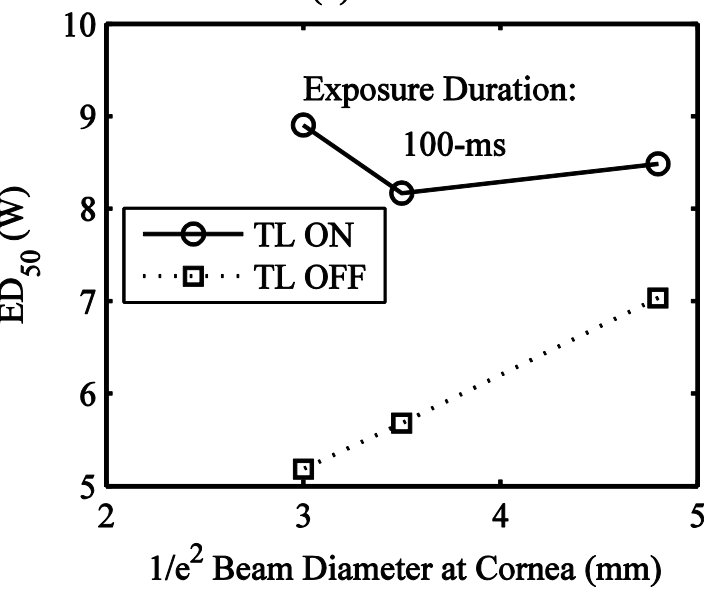

(d) $1130-\mathrm{nm}$

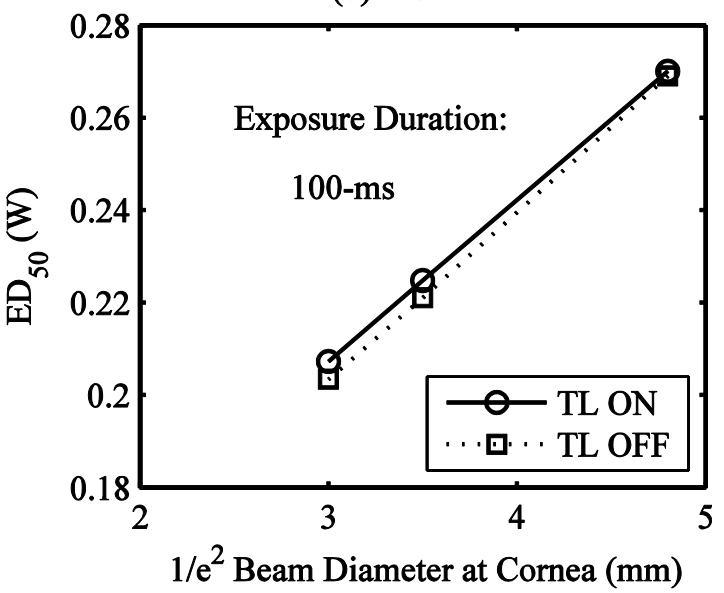

Figure 6.6: The predicted $\mathrm{ED}_{50}$ as a function of the input beam-diameter at the corneal plane. Values were determined by when the condition was able to reach $\Delta \mathrm{T}_{0}$ of $50^{\circ} \mathrm{C}$. 


\subsubsection{BTEC vs. First-Order Thermal Lensing Model}

The first-order model was compiled for cases of a 2.5 and $5-\mathrm{mm}$ beam using 0.25 and 1-W power of CW 1319-nm laser radiation delivered to the corneal plane to populate a matrix of beam-waist radii, $\omega$, as a function of time, $t$ and space, $z$. The resulting temperature rise, $\Delta \mathrm{T}$, at the surface of a retinal slab situated $1.99-\mathrm{cm}$ behind the front surface of the cornea is reported in Table 6.7 for each model and examined case.

Table 6.7: The side-by-side results from the first-order thermal lensing (FOTL) and BTEC models with and without axial conduction. The temperature rise is taken at the observational time point for a $\mathrm{CW}$ laser exposure (the laser source never turns off). The simulated eye in these computations used a homogenous $\mu_{\mathrm{a}}=1.3598 \mathrm{~cm}^{-1}$ (the value for water) and compartmentalized thermal coefficients from Table 6.1.

\begin{tabular}{|c|c|c|c|c|c|c|c|c|}
\hline \multirow[b]{2}{*}{$\begin{array}{c}\lambda \\
(\mathrm{nm})\end{array}$} & \multirow[b]{2}{*}{$\begin{array}{c}\mu_{\mathrm{a}} \\
\text { retina } \\
\left(\mathrm{cm}^{-1}\right)\end{array}$} & \multirow[b]{2}{*}{$\begin{array}{l}t_{\text {Observation }} \\
(\mathrm{s})\end{array}$} & \multirow[b]{2}{*}{$\begin{array}{l}\text { Power } \\
\text { (at } \\
\text { Cornea) } \\
\text { (W) }\end{array}$} & \multirow[b]{2}{*}{$\begin{array}{c}1 / \mathrm{e}^{2} \\
\text { Diameter } \\
\text { at } \\
\text { Cornea } \\
(\mathrm{mm})\end{array}$} & \multirow[b]{2}{*}{$\begin{array}{c}\text { Thermal } \\
\text { Lens } \\
\text { ON or } \\
\text { OFF }\end{array}$} & \multicolumn{3}{|c|}{$\begin{array}{l}\text { Retinal Temperature Rise }(\Delta \mathrm{T}) \text { from a } \\
37^{\circ} \mathrm{C} \text { Body Temp. }\end{array}$} \\
\hline & & & & & & $\begin{array}{c}\text { FOTL } \\
\text { Model (no } \\
\text { axial } \\
\text { conduction) }\end{array}$ & $\begin{array}{c}\text { BTEC } \\
\text { Model } \\
\text { axial } \\
\text { conduction } \\
\text { ON }\end{array}$ & $\begin{array}{c}\text { BTEC } \\
\text { Model } \\
\text { axial } \\
\text { conduction } \\
\text { OFF }\end{array}$ \\
\hline \multirow{8}{*}{1319} & \multirow{8}{*}{1.3598} & \multirow{8}{*}{1} & 1 & \multirow{2}{*}{5} & \multirow{4}{*}{ ON } & 5.22 & 4.12 & 4.12 \\
\hline & & & 0.25 & & & 1.33 & 1.24 & 1.23 \\
\hline & & & 1 & \multirow{2}{*}{2.5} & & 6.28 & 2.94 & 2.94 \\
\hline & & & 0.25 & & & 1.70 & 1.24 & 1.20 \\
\hline & & & 1 & \multirow{2}{*}{5} & \multirow{4}{*}{ OFF } & 5.33 & 5.32 & 5.33 \\
\hline & & & 0.25 & & & 1.33 & 1.33 & 1.33 \\
\hline & & & 1 & \multirow{2}{*}{2.5} & & 6.93 & 6.94 & 6.92 \\
\hline & & & 0.25 & & & 1.73 & 1.73 & 1.73 \\
\hline
\end{tabular}

\subsection{DISCUSSION}

The first-order thermal lensing model presented in Chapter 4 predicted the strength of the thermal lens increases with decreasing input beam-diameters at the corneal 
plane. Additionally, the experimental data of the wavelength dependency on thermal lensing presented in Chapter 3 indicated for all things being equal (equal input beamdiameter and power at the corneal plane), the 1150-nm laser radiation wavelength had the strongest thermal lens followed by 1130, 1318 and then 1110-nm. The CW NIR retinal $\mathrm{ED}_{50}$ study presented in Chapter 5 determined a scaling factor (SF) method, which neglected thermal lensing, fit experimental data very well suggesting thermal lensing was not affording protection of the retina under tested experimental conditions. With these data in consideration, the 1110-1319-nm laser radiation wavelengths were investigated in the first-order model for a series of varying input beam-diameters at the corneal plane. A brief discussion surrounding the algorithm implemented for estimating chromatic dispersion should be revisited before discussing the results from this study.

The method used for estimating chromatic dispersion required superimposing dispersion measurements between 700-900 nm wavelengths from Fernandez, Unterheuber et al. 2005 onto the model from Liou and Brennan 1997 for wavelengths less than 700-nm (see section 2.2.3 for a complete description). For this study, the linear fit to the Fernandez, Unterheuber et al. 2005 data was extended out to wavelengths as far as $1400-\mathrm{nm}$. At this time, no data exist on chromatic dispersion measurements beyond the $900-\mathrm{nm}$ wavelength in the eye. It is possible that this method overestimated the size of the beam-waist at the retina for wavelengths in the NIR such that thermal lensing had already been worked into the formula for creating the action spectra by Lund, Edsall et al. 2008, and scaling factor algorithm in Chapter 5, by pure coincidence. Given that thermal lensing is a natural phenomenon arising from the interaction of a media exposed to laser radiation, it should be stressed that it is assumed that thermal lensing occurred in the eye, to some degree, for each wavelength explored in the experimental study in 
Chapter 5. Overestimated values for the beam-waist radii delivered to the retina at $t=0$ would explain why the scaling factor method in Chapter 5 predicted the retinal $\mathrm{ED}_{50}$ data within $10 \%$ of the experimental values. However, the modeling study in this chapter has explored thermal lensing in sufficient detail by examining the temperature rise at the retina for a multivariate analysis of the phenomenon in a model eye.

Damage models which rely on the Arrhenius equation require time-temperature history to predict damage, suggesting lower peak temperatures could achieve damage given sufficient time for exposure. However, an in vitro retinal study by Denton, Foltz et al. 2009 found it is the average peak temperature achieved from the laser exposure which dictated the fate of a cell in a cell-culture exposed to $\mathrm{CW}$ laser radiation (average peak temperature was determined by the temperature averaged over the rim, or perimeter, of dead cells). The Denton, Foltz et al. 2009 data provided a lower limit (taken to be $\sim 13^{\circ} \mathrm{C}$ ) while data from Pearce and Thomsen 1995 provided an upper limit (taken to be $\left.\sim 50^{\circ} \mathrm{C}\right)$ for the threshold temperatures $\left(\Delta \mathrm{T}_{0}\right)$ used to establish the damage endpoint used in this study. The experimental input parameter conditions from Table 6.4 were then implemented in the first-order thermal lensing model (with thermal lensing on) and the values for $\mu_{\mathrm{a}}$, used for the retina, corresponding to the upper and lower limits of $\Delta \mathrm{T}_{0}$ were determined for each wavelength (Table 6.6). There is a $\mu_{\mathrm{a}}$ for the retina for each wavelength to allow for independent analyses of the two opposing hypotheses; 1 - that thermal lensing occurred, but was not significant enough to protect the retina for large beam-diameters delivered to the cornea and 2 - that thermal lensing did not occur for large or small beam-diameters delivered to the cornea.

Section 2.2.1 detailed how the $\mu_{\mathrm{a}}$ of the retinal pigment epithelium, RPE, was expected to be on the order of that of water such that the absorption of water would 
contribute significantly to the absorption of laser radiation at this wavelength in the retina. The value for $\mu_{\mathrm{a}}$ of the RPE, found by fitting an equation to data from Birngruber, Hillenkamp et al. 1985 was 97.9 and $37.5 \mathrm{~cm}^{-1}$ for 1110 and 1319-nm laser radiation respectively, substantially larger than that of water; $\mu_{\mathrm{aw}}=0.345$ and $1.36 \mathrm{~cm}^{-1}$ for respective wavelengths. The ratio of RPE $\mu_{\mathrm{a} 1110 \mathrm{~nm}}: \mu_{\mathrm{a} 1319 \mathrm{~nm}}$ is $2.6: 1$ from the values used in Chapter 5 (based on Birngruber, Hillenkamp et al. 1985) and 0.25:1 for water (based on Hale and Querry 1973). In this study, the retina was assumed to be uniformly absorbing, due to both water and pigmentation, and $\mu_{\mathrm{a}}$ was determined upon achieving $\Delta \mathrm{T}_{0}$ for an experimental input reference. This gave a ratio of $\mu_{\mathrm{a} 1110 \mathrm{~nm}}: \mu_{\mathrm{a} 1319 \mathrm{~nm}}$ of $2.3: 1$ with thermal lensing on for both the upper and lower limits placed on $\Delta \mathrm{T}_{0}$ (see Table 6.6). These ratios suggest the scaled $\mu_{\mathrm{a}}$ values for each wavelength were reasonable relative to one another. The time to reach $\Delta \mathrm{T}_{0}$ and $\mu_{\mathrm{a}}$ used for the retina were found to be independent as long as the threshold $\Delta \mathrm{T}_{0}$ was adjusted accordingly. This independent relationship means that the data presented after Table 6.6 which were based upon the upper limit of $\Delta \mathrm{T}_{0}\left(50^{\circ} \mathrm{C}\right)$ would yield the same results if they had been evaluated using the lower limit of $\Delta \mathrm{T}_{0}$, just scaled down accordingly (input power and exposure time were independent of the selected $\Delta \mathrm{T}_{0}$ and corresponding $\mu_{\mathrm{a}}$ ).

Data in the study for this chapter were evaluated under two hypotheses: 1 Thermal lensing occurred in the ocular media, but did not afford protection of the retina for the experimental condition tested in Chapter $5\left(\mathrm{dn} / \mathrm{dT}=-8.92 \mathrm{~K}^{-1}\right)$. This afforded an analysis from the perspective on how the time-temperature dependency was related to radial thermal diffusion at the retina, input beam-diameter and power delivered to the cornea and the subsequent thermal lens. 2 - Thermal lensing was not occurring in the ocular media $(\mathrm{dn} / \mathrm{dT}=0)$. This afforded an analysis from the perspective on how the 
time-temperature dependency was related only to radial thermal diffusion at the retina, and input beam-diameter and power delivered to the cornea.

The first-order thermal lensing model shows how thermal lensing caused the beam-waist radius, $\omega$, to increase with time (Fig. 6.2) and the source term to decrease in time at the retina (Fig. 6.3) for large (5-mm) and small (3-mm) beam diameters delivered to the corneal plane, whereas, cases without thermal lensing maintained a constant $\omega$ and source term value at the retina over time. Fig. 6.5a showed how for equivalent absorption at the retina and damaging threshold endpoints, the 5-mm beam-diameter case reached $\Delta \mathrm{T}_{0}$ in $0.08 \mathrm{~s}$ and $\sim 0.05 \mathrm{~s}$ for the cases with and without thermal lensing respectively. In fact, the power would have to be reduced from $14.5 \mathrm{~W}$ to approximately $11.3 \mathrm{~W}$ in order for the $5-\mathrm{mm}$ case without thermal lensing to reach $\Delta \mathrm{T}_{0}$ at $0.08 \mathrm{~s}$ (this is depicted in Fig. 6.6). Though the temperature rise was faster for cases without thermal lensing at 1319$\mathrm{nm}$, once the source term was turned off, the temperature dissipated more slowly when the thermal lens was on compared to when it was turned off. This is due to the fact that the temperature was taken to cool at a rate of

$$
\Delta T_{\text {Cooling }}=\Delta T_{\text {Peak }} \exp \left(-\eta \Delta t / \omega^{2}\right) \quad \text { Eq. } 6.3
$$

where $\Delta \mathrm{t}$ is the time since the laser source turned off and $\omega$ is the beam-waist radius at the retina at the time the laser radiation source turned off (van Gemert and Welch 1995). Obviously, the value of $\omega$ increases the longer the source is left on, requiring more time to dissipate heat, or longer for $\Delta \mathrm{T}$ at $\mathrm{r}=0$ to relax back to initial state, compared to a case with no thermal lensing where $\omega$ remains constant over time. In the case of the 3-mm beam diameter in Fig. 6.5b, the case with no thermal lensing reached the threshold temperature in $\sim 17-\mathrm{ms}$, whereas the case with thermal lensing never reached the threshold temperature in the $80-\mathrm{ms}$ exposure, but took much longer to dissipate the 
thermal energy. The 3-mm case depicted in Fig, 6.5b would require a $\mathrm{CW}$ exposure of $\sim 150$-ms to reach threshold temperature.

The predicted retinal thresholds as a function of input beam diameter at the cornea presented in Fig. 6.6 were determined by when, in time, the peak temperature was achieved in a $\mathrm{CW}$ exposure. The data in Fig. 6.6 show a linear relationship between the input-beam diameter delivered to the corneal plane and $\mathrm{ED}_{50}$, for all wavelengths, when the thermal lens was turned off. With the thermal lens on, the predicted $\mathrm{ED}_{50}$ values only began to significantly separate out from the cases without thermal lensing for the 1310 and 1319-nm wavelengths.

From Chapter 5, the experimental upper and lower fiducial limits of the 1319-nm wavelength, 80-ms exposure, 5-mm diameter were 16.2 and $12.5-\mathrm{W}$ respectively. The single highest and lowest input powers for the 1319-nm, 5-mm, 80-ms exposures in Chapter 5 which yielded no lesion and a lesion respectively (i.e. the highest exposure which resulted in no lesion, and the lowest exposure which resulted in a lesion) were 22 and $11.65 \mathrm{~W}$ respectively. This range covered by the experimental $1319-\mathrm{nm}$ retinal $\mathrm{ED}_{50}$ data represents the variation imposed on threshold data due to biological variability such as eye length. No two subject's retinas or the two eyes in a single subject are identical. In lieu of these facts, the 1319-nm wavelength, 80-ms exposure, 5-mm diameter simulation results in Fig. 6.6a suggested that the 1319-nm, 5-mm input beam diameter case with thermal lensing needed $\sim 28 \%$ more power delivered to the retina in order to achieve the same damage end point for the case of no thermal lensing tested under equal conditions. Knowing that the 1319-nm wavelength, 80-ms exposure, 5-mm diameter experimental $\mathrm{ED}_{50}$ was $14.5-\mathrm{W}$ and assuming thermal lensing was occurring for this experimental measurement, then only $\sim 11.3-\mathrm{W}$ would be needed to create retinal damage 
without thermal lensing. This $\mathrm{ED}_{50}$ value of $11.3-\mathrm{W}$ is little more than $1-\mathrm{W}$ lower than the lower fiducial limit $(12.5-\mathrm{W})$ of the experimental results from the respective data set. Though 11.3-W is outside, it is not significantly outside the range of experimental retinal lesions for this tested case. It is possible that a $28 \%$ difference in power is not sufficient to separate out thermal lensing effects from biological variability at these wavelengths. Sorting out thermal lensing effects from the experimental in vivo results is further complicated by the uncertainty in the initial beam-waist delivered to the retina due to chromatic dispersion. However, examining cases where the thermal lens was predicted to be maximized may allow for separation of these uncertainties from thermal lensing effects.

The strongest thermal lens case for the 1319-nm wavelength was the 3-mm beam diameter, Fig. 6.6a, which required an input power of $\sim 20-\mathrm{W}$ to achieve threshold temperature for an 80-ms exposure. All other power levels for the remaining tested input beam diameters for the $1319-\mathrm{nm}$ wavelength fell in between the $14.5-\mathrm{W}$ retinal $\mathrm{ED}_{50}$ threshold and this upper limit of $\sim 20-\mathrm{W}$. The 1319-nm, 3-mm beam diameter and $80-\mathrm{ms}$ results predicted a retinal $\mathrm{ED}_{50}$ of 20 and $7.9-\mathrm{W}$ with and without thermal lensing respectively (see Fig. 6.6a). Given biological variability between subjects and the fact

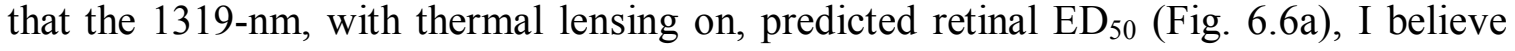
thermal lensing would afford protection of the retina by no more than a factor of $\sim 2-3$ for exposure durations between 80-100-ms for wavelengths between 1300-1340-nm for smaller beam-diameters delivered to the corneal plane. Without thermal lensing, a CW NIR laser radiation exposure is predicted to become more dangerous (decreasing $\mathrm{ED}_{50}$ ) to the retina with decreasing input-beam diameter delivered to the cornea. Results in Fig. 6.6 c-e suggested that biological variability would overshadow any thermal lensing effect 
in the eye for wavelengths less than 1300-nm. The parameter sets which exhibited significant thermal lensing in this study should be compiled in higher fidelity models for validation. All data sets in this study were compiled for a relaxed, unaccommodated rhesus eye with chromatic dispersion.

In comparing the BTEC model computations for $\mathrm{T}(\mathrm{t})$ to the first-order thermal lensing model, it is evident that the temperature calculation used in the first-order model gave similar results to the higher fidelity BTEC model with or without axial conduction under the simulated conditions (Table 6.7). This validated the algorithm employed to calculate $T(t)$ in the first-order thermal lensing model. Differences in the results with thermal lensing on between the BTEC model and the first-order thermal lensing model arise from inaccuracies between the beam-waist radii, $\omega$, as a function of time at the retina. The BTEC model estimated the values of $\omega_{\text {retina }}$ based upon $\omega_{\min }$ as a function of time. The BTEC model assumed a linear relationship between the input beam at the cornea and the focus at $\omega_{\min }$. However, the thermal lens is strongly non-linear, not only shifting the focus in the z-direction, but expanding the beam profile at every step along the z-axis as a function of power density delivered to the corresponding z-position. Thus, the BTEC model used an independent fitting algorithm to estimate the $\omega_{\text {retina }}(t)$ lending to some of the differences between the two models results. Notice the cases in Table 6.7 without thermal lensing, when the beam-waist radii were constant, demonstrated that the two models obtained the same results. The cases in Table 6.7 ultimately illustrated how axial conduction does not greatly contribute to the temperature achieved at the retina if the thermal and optical properties of the retina and vitreous are very close or the same. If the thermal coefficients and linear absorption coefficients between the vitreous and retina 
began to differ significantly, then axial conduction did begin to play a larger role in the temperature achieved at the retinal surface.

Results from comparing the two models suggest the BTEC model should use full propagation methods to more accurately predict the $\omega(\mathrm{z}=$ retina, $\mathrm{t})$ and the subsequent $\Delta \mathrm{T}$. Full beam-propagation methods are currently being incorporated into the BTEC model at the Air Force Research Lab (Thomas, Vincelette et al. 2007; Zohner, Clark et al. 2008).

\subsection{CONCLUSION}

Assuming no thermal lensing, and that damage occurred when a threshold temperature increase of $50^{\circ} \mathrm{C}$ was achieved, model results predict a linear relationship between the $\mathrm{ED}_{50}$ (Watts delivered to the corneal plane) and $1 / \mathrm{e}^{2}$ beam-diameter for equivalent exposure durations. Assuming thermal lensing occurred in ocular media for CW NIR retinal exposures, the modeling and simulation efforts from this study predict that thermal lensing would cause this linear relationship to break for smaller $1 / \mathrm{e}^{2}$ beam diameters $(<\sim 4-\mathrm{mm})$ at weakly transmitted wavelengths $(<\sim 5 \%)$ and $\mathrm{ED}_{50}$ 's would be expected to be higher than the linear estimate.

Modeling results for CW NIR retinal damage thresholds for large beam diameters (5-mm) delivered to the corneal plane predicted that power inputs would differ by as much as $28 \%$ between cases with and without thermal lensing. Though $28 \%$ appears to be a large increase, it may not be sufficient to significantly overshadow the uncertainty with the retinal threshold $\mathrm{ED}_{50}$ in regards to biological variability under this input beam condition (5-mm 1/ $\mathrm{e}^{2}$ beam diameter). Furthermore, the chromatic dispersion models used to fit CW NIR retinal threshold data may unknowingly account for some thermal 
lensing by overestimating the beam-waist radius at $t=0$ for NIR wavelengths. As the $1 / \mathrm{e}^{2}$ input-beam diameter delivered to the cornea was decreased, the variation between the predicted damage threshold for cases with and without thermal lensing began to become substantially more pronounced by as much as a factor of 2.5 fold increase in the power needed to reach the damage threshold between equivalent cases with and without thermal lensing. Regardless of the uncertainties with respect to biological variability and chromatic dispersion estimates, results from this study have suggested that the retinal $\mathrm{ED}_{50}$ for smaller beam-diameters delivered to the corneal plane will be greater than 14.5W given the nature of the thermal lens for the 1319-nm wavelength (80-ms exposure durations).

The first-order thermal lensing model can be used to predict the temperature rise at the retina for a given parameter set using integration methods from Matlab. If the thermal and linear absorption coefficients of the retina and vitreous are assumed to be fairly uniform, and the damage end-point is assumed to be a temperature threshold condition then the first-order model does not significantly differ from the higher fidelity models. The first-order thermal lensing model cannot use a multi-layer retinal model at this time. Higher fidelity models may be more robust than the first-order thermal lensing model, but they require more computational power and time. The first-order thermal lensing model is a good method for examining a large range of parameter sets for $\mathrm{CW}$ NIR laser radiation in an eye to guide specific sets for examination using higher fidelity models. 


\subsection{ACKNOWLEDGEMENTS}

The author would like to thank Dr. Robert Thomas, with the Air Force Research Lab, and Dr. Michael Denton, with Northrop Grumman, for their assistance with this chapter. 


\section{CHAPTER 7}

\section{OBSERVATIONAL METHOD FOR QUANTIFYING THERMAL LENSING USING ADAPTIVE OPTICS}

\subsection{ABSTRACT}

Adaptive optics has become a useful technology to improve retinal imaging by correcting ocular aberrations found in the wavefront returning from the retina. This technology is suited for describing the aberration types existing on a wavefront. An adaptive optics system built by Iris AO, Inc., is used to examine the aberrations induced in a water-filled Cain-cell artificial eye by a thermal lens from a $500-\mathrm{mW}$, continuouswave, 1150-nm laser radiation wavelength source. Data show how thermal lensing in the

eye involves several aberrations: defocus, astigmatism, $3^{\text {rd }}$ order coma, $3^{\text {rd }}$ order sphere and trefoil.

\subsection{INTRODUCTION}

The formation of the thermal lens was demonstrated in a water-filled artificial eye in Chapter 3. The same chapter included a presentation of data to illustrate the thermal lens in both vitreous and water-filled samples exposed to identical optical conditions; finding the thermal lens in each of the two samples were remarkably similar. Data from Chapter 3 have answered several questions surrounding the strength of the thermal lens with wavelengths in the near-infrared as a function of power and time. Modeling data from Chapters 4 and 6 provided information on how the thermal lens was also dependent upon the input geometry of the beam at the corneal plane. Together, these data assisted 
in understanding the laser-tissue interaction of thermal lensing in an eye; however, they did not describe the types of aberrations involved with the thermal lens or demonstrate the phenomenon in vivo.

The eye is a very complex structure, capable of accommodating as much as 10 Diopters (D) (Wald and Griffen 1947; Westheimer 1980; Atchison and Smith 2002; Roorda 2002; Artal, Benito et al. 2006). The muscles supporting the eye in the occipital socket provide the structure for the optical configuration needed to observe the thermal lensing effect. Placing a confocal imaging system at the retinal plane of an animal model is not only difficult, but highly invasive. When the eye is removed from the socket, it is susceptible to gravitational pull; rendering the eye useless without sophisticated mechanical support to reconstruct the eye's physiological conditions. Given the inherent difficulties with imaging the thermal lensing effect in vivo, it is worthwhile to propose a non-invasive method for observing the thermal lens in an animal model.

Adaptive optics (AO) is a growing field of technology designed to improve the imaging capabilities of optical systems. AO refers to an optical systems capability to "adapt" to optical aberrations through a feedback loop to correct for induced wavefront aberrations. In the field of biomedical engineering, the wavefront aberrations are typically introduced from a biological sample. Liang et al. used an AO system to examine the wavefront aberrations in a human eye in 1994 and Mansell et al. used an AO system to examine the thermal lensing effect in optical glasses, but an AO system has never been used to quantify the thermal lensing effect in an eye (Liang, Grimm et al. 1994; Mansell, Hennawi et al. 2001).

In this chapter, an adaptive optics system is used to demonstrate how the thermal lensing effect can be quantified in a system designed for observing the wavefront in a 
rhesus eye. AO data from the Zernike polynomials describing the types of aberrations in the sample are presented for thermal lensing in a water-filled Cain-cell artificial eye exposed to a 500-mW continuous-wave, CW, 1150-nm laser radiation wavelength source. These data are used to demonstrate how the technique can be extended to an animal model; observing the thermal lens in real-time in vivo without injury to the subject.

\subsection{BACKGROUND}

The history of adaptive optics can be traced back with ties to both astronomy and National Defense research efforts (Marquet and Hardy 1978). A typical AO system uses a Shack-Hartmann wave-front sensor. The Shack-Hartmann system is a well known optical wavefront measurement technique which employs the use of a lenslet array, constructed from the Scheiner disc, and a CCD camera (Liang, Grimm et al. 1994; Thibos 2000; Mansell, Hennawi et al. 2001; Thibos 2001). A beam of light is brought to a focus at the fundus of the eye; the reflected wavefront from the fundus is measured by the Shack-Hartmann aberrometer which captures aberrations of the eye (Thibos 2000). In 1619, Scheiner discovered by placing two pinholes, one centered and one on the peripheral, on a disc in front of the eye, a point source of light, such as a star, will form two images at the retina if the person's eye is imperfect. Scheiner recognized that a lens with the appropriate power could correct the double image problem. In 1900, Hartmann expanded the Scheiner disc concept by creating a screen of pinholes. Each pinhole in the Hartmann screen isolates a small portion of the impending wavefront emerging from the eye. The local slope of each wavefront relative to the known position of the pinhole from the Hartmann screen can then be determined (see Fig. 7.1). In 1972, Shack further built 
upon the Hartmann-Scheiner concept by changing the screen of pinholes into an array of tiny lenses. This way, a CCD sensor could be positioned at a known distance from the lenslet array and each lenslet could focus an isolated portion of the emerging wavefront onto the sensor. Shack's contribution to the aberrometer improved the signal to noise ratio. Each lens creates a Fraunhofer diffraction pattern at the sensor. If the impending wavefront were perfect, the series of diffraction patterns created by the lenslet array appears as a matrix of spots (Fig. 7.1a). Aberrations from the eye distort the impending wavefront causing the matrix of spots to become distorted (Fig. 7.2b). The slope from each ray of light from a lens within the array determines the spot position on the CCD sensor, also deemed the Shack-Hartmann Wavefront Detector (SHWD).
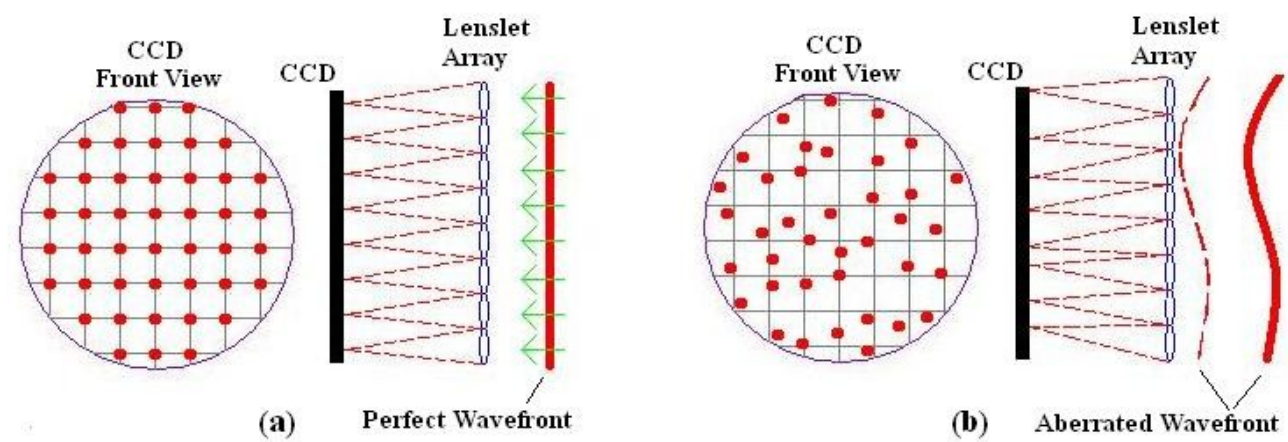

Figure 7.1: (a) A perfect wavefront passes through the lenslet array in a Shack-Hartmann system. The result is an ideal configuration of spots on the CCD sensor. (b) An imperfect wavefront passes through the lenslet array. Notice the slope of the cone angles depicted in a and b; the location of the spot on the CCD grid helps to determine the incoming angle (translated into a slope) of the beam-segment from the distorted wavefront. These slopes are used to reconstruct the wavefront at the image plane. The segmented wavefront represents how each lens in the array takes in only a small segment of the wavefront and brings that portion of the wavefront to focus at some position on the CCD sensor. The position where the wave segment is focused on the CCD is relative to the slope the light segment forms as it exits the individual lens.

The purpose of the relay lenses depicted in Fig. 7.2, is to focus the lenslet array onto the pupil (Thibos 2000). These lenses allow for pupil-plane to be imaged directly onto the lenslet array. This is done to cause the wavefront to immediately subdivide into 
segments as light leaves the eye. The shape of the wavefront describes the optical limitations of the eye in terms of forming a perfect image.

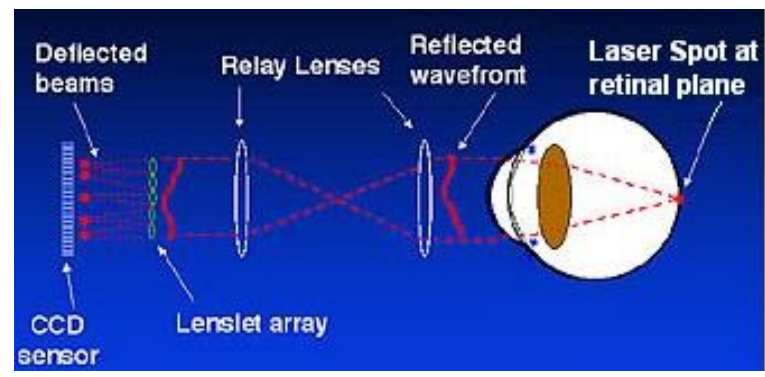

Figure 7.2: Basic optical configuration of a Shack-Hartmann system to measure the wavefront aberrations associated with the eye (Thibos 2000).

In order to dynamically correct a distorted wavefront coming from the eye, a software algorithm must interpret the aberrations of the wavefront detected on the CCD sensor. Once the aberrations are determined, the software provides feedback to an optical element to dynamically correct the aberrated wavefront; this is commonly referred to as "closing the loop" in the AO system. An optical element capable of this dynamic interaction is a deformable mirror (DM) with a response time tied in with the control loop of the system design (typically 15-30 Hz). To achieve this, the DM must first be calibrated to a reference wavefront, correcting for any aberrations in the system before placing a sample into the path, typically a calibration optic is used to establish a baseline. Once the system is calibrated, an eye can be placed into the system. The wave-front reflected off the retina will be distorted relative to the wave-front launched into the eye at the corneal plane. The wave-front sensor must interface with software to calculate the necessary parameters to correct for the aberrations induced by the sample. This is the wave-front feedback loop as shown in Fig. 7.3. 


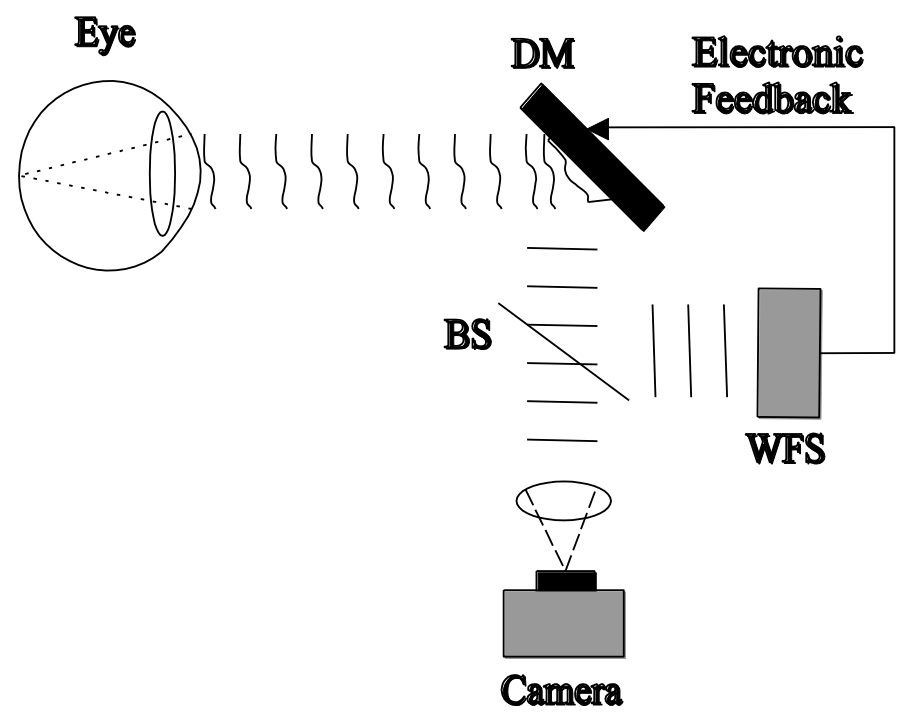

Figure 7.3: A typical wave-front feedback loop for an adaptive optics (AO) ocular imaging system. (DM - Deformable Mirror, BS - Beam Splitter, WFS - Wavefront Sensor). Currently, AO feedback loops update the image and DM position at a rate from $15-30 \mathrm{~Hz}$.

The types of aberrations which are detected by the wavefront sensor are described by the Zernike polynomial; a set of orthogonal polynomials used to describe the wavefront function arising from optical systems with a circular aperture (pupil) (Yeh and Azar 2005; Weisstein 2009). Typical AO systems for the eye calculate as many as 21 of the coefficients used to comprise the Zernike polynomial, also called the mode, which describes the wavefront detected by the wavefront sensor at a point in time. Each Zernike polynomial coefficient, or mode, correlates with a specific aberration type such as defocus, astigmatism, and trefoil. The Zernike polynomial associated with its specific aberration type has become commonly represented in the "Periodic Table of Zernikes" (Thibos 2001). For example, modes 1-5 represent the aberration types of piston, tip, tilt, astigmatism ( $45^{\circ}$ axis), and defocus respectively. The Zernike polynomial coefficient associated with each of the aberration types is given in Table 7.1. The wavefront shape corresponding to each Zernike polynomial, or mode, is shown in Fig. 7.4. 


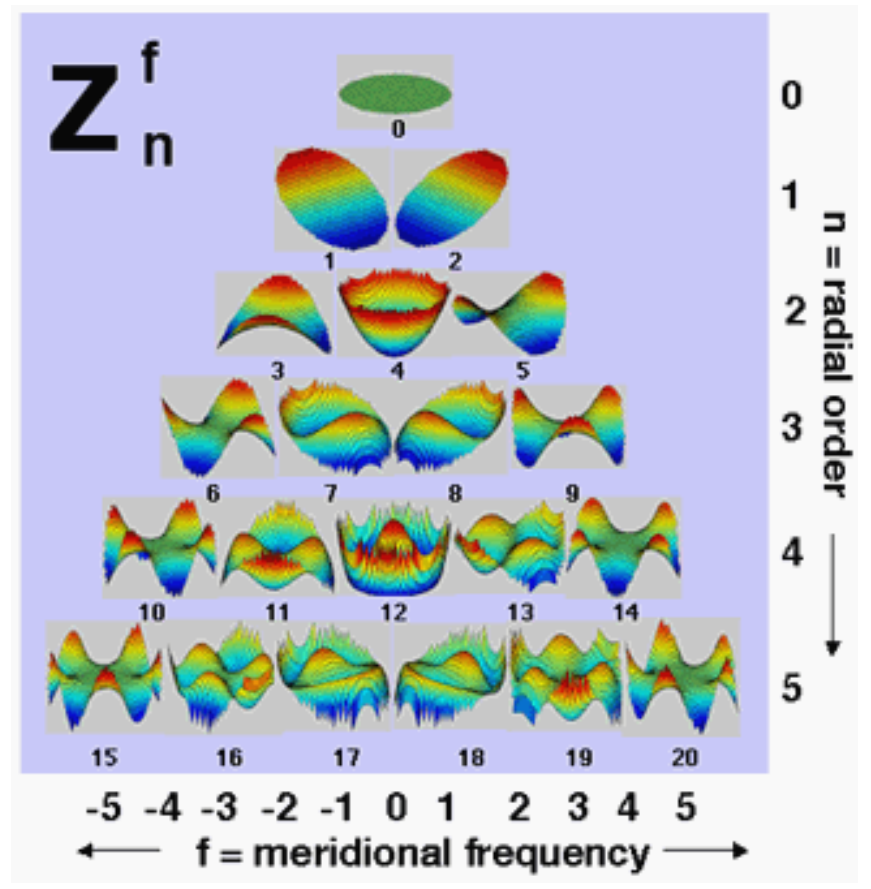

Figure 7.4: The periodic table of Zernike polynomials represented as respective wavefront shapes. See Table 7.1 for the type of aberration corresponding with each mode. Figure from Thibos 2001.

In a large population study on the optical quality of the human eye, Thibos et al. reported the average human eye is not free of aberrations (Thibos, Hong et al. 2002). The study was based upon 200, ocular disease-free subjects. It is common for an eye to have pre-existing lower-order aberrations (defocus and astigmatism) which can be corrected with glasses or contact lenses. Higher-order aberrations (mode $>2$ ) occurred in several subjects. Thibos, Hong et al. 2002 reported it was rare to find a single, individual eye where any aberration coefficient was zero at any of the Zernike modes. 
Table 7.1: The aberration types representing the Zernike polynomial coefficients (beginning with 0) listed in their order of appearance in the Iris AO system's algorithm.

\begin{tabular}{|c|c|c|c|}
\hline $\begin{array}{c}\text { Mode } \\
\text { (Order of } \\
\text { Appearance } \\
\text { in Iris AO } \\
\text { File) }\end{array}$ & $\begin{array}{c}\mathrm{n} \\
\text { (Radial } \\
\text { Order) }\end{array}$ & $\begin{array}{c}\mathrm{f} \\
\text { (Meridional } \\
\text { Frequency) }\end{array}$ & $\begin{array}{c}\text { Zernike } \\
\text { Coefficient's } \\
\text { Aberration } \\
\text { Type }\end{array}$ \\
\hline 0 & 0 & 0 & Piston \\
\hline 1 & 1 & -1 & Tip \\
\hline 2 & 1 & 1 & Tilt \\
\hline 3 & 2 & -2 & $\begin{array}{c}\text { Astigmatism, } \\
45^{\circ} \text { Axis }\end{array}$ \\
\hline 4 & 2 & 0 & Defocus \\
\hline 5 & 2 & 2 & $\begin{array}{c}\text { Astigmatism, } \\
90^{\circ} \text { Axis }\end{array}$ \\
\hline 6 & 3 & -3 & Trefoil \\
\hline 7 & 3 & -1 & $\begin{array}{c}3^{\text {rd }} \text { Order } \\
\text { Vertical } \\
\text { Coma }\end{array}$ \\
\hline 8 & 3 & 1 & $\begin{array}{c}3^{\text {rd }} \text { Order } \\
\text { Horizontal } \\
\text { Coma }\end{array}$ \\
\hline 9 & 3 & 3 & Trefoil \\
\hline
\end{tabular}

\begin{tabular}{|c|c|c|c|}
\hline $\begin{array}{c}\text { Mode } \\
\text { (Order of } \\
\text { Appearance } \\
\text { in Iris AO } \\
\text { File) }\end{array}$ & $\begin{array}{c}\mathrm{n} \\
\text { (Radial } \\
\text { Order) }\end{array}$ & $\begin{array}{c}\mathrm{f} \\
\text { (Meridional } \\
\text { Frequency) }\end{array}$ & $\begin{array}{c}\text { Zernike } \\
\text { Coefficient's } \\
\text { Aberration } \\
\text { Type }\end{array}$ \\
\hline 10 & 4 & -4 & Quadrafoil \\
\hline 11 & 4 & -2 & $\begin{array}{c}2^{\text {nd }} \text { Order } \\
\text { Astigmatism }\end{array}$ \\
\hline 12 & 4 & 0 & $\begin{array}{l}3^{\text {rd }} \text { Order } \\
\text { Spherical }\end{array}$ \\
\hline 13 & 4 & 2 & $\begin{array}{c}2^{\text {nd }} \text { Order } \\
\text { Astigmatism }\end{array}$ \\
\hline 14 & 4 & 4 & Quadrafoil \\
\hline 15 & 5 & -5 & Pentafoil \\
\hline 16 & 5 & -3 & $\begin{array}{c}\text { 2nd Order } \\
\text { Trefoil }\end{array}$ \\
\hline 17 & 5 & -1 & $\begin{array}{c}2^{\text {nd }} \text { Order } \\
\text { Coma }\end{array}$ \\
\hline 18 & 5 & 1 & $\begin{array}{c}2^{\text {nd }} \text { Order } \\
\text { Coma }\end{array}$ \\
\hline 19 & 5 & 3 & $\begin{array}{l}\text { 2nd Order } \\
\text { Trefoil }\end{array}$ \\
\hline 20 & 5 & 5 & Pentafoil \\
\hline
\end{tabular}

The aberrations of the average eye have been explored, but the aberrations arising from a thermal lens in an ocular geometry have never previously been reported. In this chapter, an adaptive optics, confocal laser scanning ophthalmoscope developed by Iris, $\mathrm{AO}$, Inc., is used to image the wave-front distortions in a water-filled Cain cell artificial eye exposed to near-infrared laser radiation. The Zernike polynomial coefficients' 
transient and stead-state responses to the thermal lens are presented to provide information on the aberration types which arise due to continuous-wave near-infrared laser radiation.

\subsection{MATERIALS AND METHODS}

The AO system developed for the Air Force Research Laboratory, Optical Radiation Branch, by Iris AO, Inc., Berkley, CA, under contract number FA8650-05-C6551 was used in this experiment. Though some of the details for this system are proprietary, the general functionality of the system's specifications and performance are presented in sufficient detail. Only the AO portion of this imaging system is needed for this experiment.

The Iris AO system was first calibrated using MEB-3 and MEB-1 (there are four calibration boards, called MEB-X, specific to the system's design). MEB-3 consists of a simple target mounted at a specific distance on the board such that it coincides with the pupil plane position to check the systems alignment and ensure that a 7.2-mm diameter spot of light is created at the pupil plane. MEB-1 consists of an SF11 lens at the pupil plane and target at the "retinal" plane, and is designed for a rhesus eye configuration to assist with checking the system's alignment at the retinal plane. Since MEB-1 is dry and cannot hold a liquid sample, MEB-1 was used only for calibration purposes and should not be confused with the sample holder, the Cain-cell artificial eye, which also mimics the rhesus eye, but is designed to hold water.

Once calibrated, a water-filled Cain-cell artificial eye (Fig. 3.2) was placed such that the o-ring forming the seal on the Cain-cell lens mount was in focus (the pupil plane position). A 635-nm laser diode was coupled into a single mode optical fiber, then 
collimated and sent into the optical system for delivery to the pupil plane. An artificial retina was made from a glass-cover slip, sprayed with artists-glass white frosting to create a textured surface, and then sprayed with flat-black paint and finally another dusting of white frosting. The artificial retina was placed into the Cain-cell and carefully positioned inside the water-filled chamber such that its textured surface was in focus (the retinal plane). With the Cain-cell lens' o-ring and artificial retina in focus (remember these focal planes were determined by the MEB calibration boards), the sample was determined to be aligned with the system sufficiently for data acquisition. The reflected wave-front returning from the eye was then read by the WFS off of the DM. The DM (manufactured by Iris AO) was an S37-X with 37 segments controlled by piston, tip, and tilt commands. The wave-front sensor (WFS) was made of two components; a Uniqvision UP-680-CL-CCD and a lenslet array AOA 0600-19-H. The WFS interfaced with a PC workstation (Dell, Precision 670) equipped with a Matrox, SOLIOS CL frame grabber card to capture the data from the WFS. Data were acquired at 30 frames per second; a typical acquisition rate for AO systems. WFS data were then processed on the PC with an adaptive optics control algorithm. The results from this algorithm were sent to a high speed National Instruments PCI-DIO-32HS I/O board to interface with the driver electronics (SmartDriver developed by Iris AO) to control the DM.

In the AO loop controller algorithm, the 21 Zernike coefficients were recorded in a text file at a rate of $30-\mathrm{Hz}$. This means the thermal lensing effect could be recorded at a rate of 30 frames per second (fps). Given the fast response time of the thermal lens results in Chapter 3, on the order of $100-\mathrm{ms}$, and the limited $30-\mathrm{Hz}$ sampling rate of the AO system, the data acquired for this study are exposed sufficiently long to reach steadystate thus minimizing risks for aliasing. The Zernike coefficients were saved and 
imported into a Matlab script (developed by Iris AO) to reconstruct the wave-front as a function of time. The Matlab script re-created the wave-front and respective point-spread function (PSF) in 2-D and 3-D from the pupil plane for a desired point in time. The 21 Zernike polynomial coefficients recorded in the Iris AO system represented the wavefront aberration types in Table 7.1.

A Coherent-OPO laser was tuned to $1150-\mathrm{nm}$ and co-aligned with the 633-nm Iris AO system's source with a beam-splitter (BS1-1240-98-1012-45UNP) to deliver a nearinfrared laser to the sample while imaging. A 1-cm diameter Uniblitz shutter was used to control the delivery of the near-infrared laser beam to the sample.

With the OPO-laser beam blocked, the AO system was activated and left open (meaning the loop was not correcting for aberrations), initiating the acquisition of Zernike coefficients over time at a rate of $30-\mathrm{Hz}$. The first few frames correspond to the initial-state of the water-filled Cain-cell artificial eye; demonstrating the aberrations introduced into the system from the Cain-cell sample. The AO system loop was then closed to correct for the wave-front aberrations at initial-state (without any thermal lensing), causing the wave-front to flatten to a non-aberrated form. The DM was then locked into this position, allowing for any further changes in the wave-front to be attributed to the thermal lens, and not the optical components of the system or sample. With the DM locked at the initial-state position, the 1150-nm laser beam was opened and exposed to the Cain cell for several seconds to reach steady-state. Deformations of the wave-front under this condition are due to the thermal energy induced in the sample giving way to the thermal lens and convectional flow. Data are reported as figures depicting the 2D and 3D wave-fronts and point-spread functions (PSF) from the pupil plane. 


\subsection{RESULTS}

The reconstructed wavefronts from the Cain-cell artificial eye, reflected from the initial state with corrected aberrations (DM activated then locked in this position), and at steady-state from thermal lensing (thermal lens induced by a CW 1150-nm wavelength with $\sim 500-\mathrm{mW}$ ) are shown in Fig. $7.5 \mathrm{a} \& \mathrm{~b}$, and $\mathrm{c} \& \mathrm{~d}$ respectively. Initial state means the near-infrared laser source is blocked.
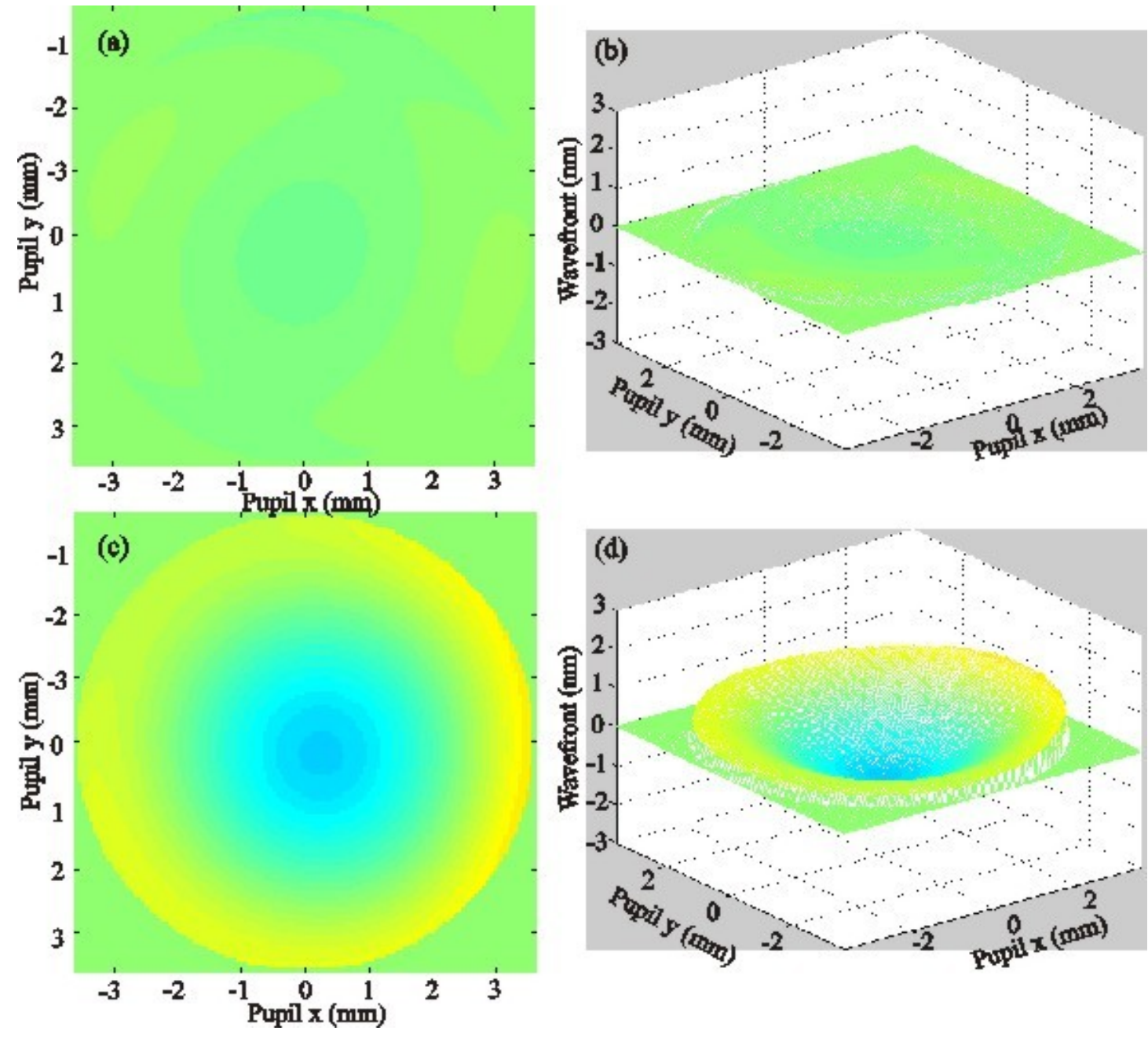

Figure 7.5: The reconstructed wavefront for the Cain-cell sample with (a \& b) the wavefront corrected, locked in place and no thermal lensing, and (c \& d) the aberrations 
resulting purely from the induced thermal lens (shown after the 1150-nm laser has been continuously exposed to the sample for $\sim 5.3 \mathrm{~s}$. The laser was blocked after $8.25 \mathrm{~s}$ ).

The Iris AO system also reconstructs the point-spread function (PSF) for each respective wavefront reconstructed from the Zernike polynomials. The corresponding PSF frames to the wavefronts shown in Fig. 7.5 are shown in Fig. 7.6.
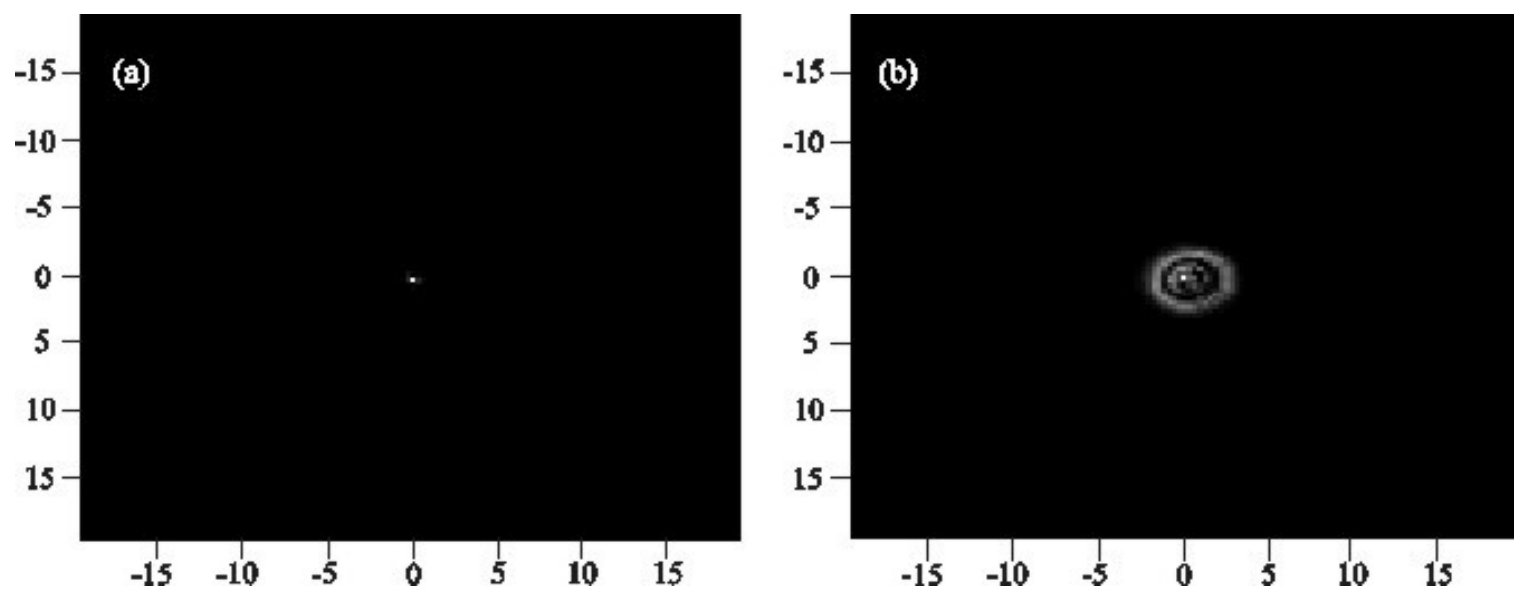

Figure 7.6: The corresponding point-spread function (PSF) for the reconstructed wavefronts depicted in Fig. 7.5. In (a) - the initial-state PSF, (b) - the steady-state, thermal lens induced PSF.

The Cain-cell lens was not free from aberrations at initial-state with a flat DM. A flat DM means the Iris AO system was not correcting for any optical aberrations in the sample. The DM was locked to at initial-state to correct for aberrations from the Caincell lens before collecting thermal lensing data; the aberrations in Fig. $7.5 \mathrm{c} \& \mathrm{~d}$ are only from the thermal lens. Aberrations from the Cain-cell lens, without thermal lensing, are shown in Fig. 7.7. 

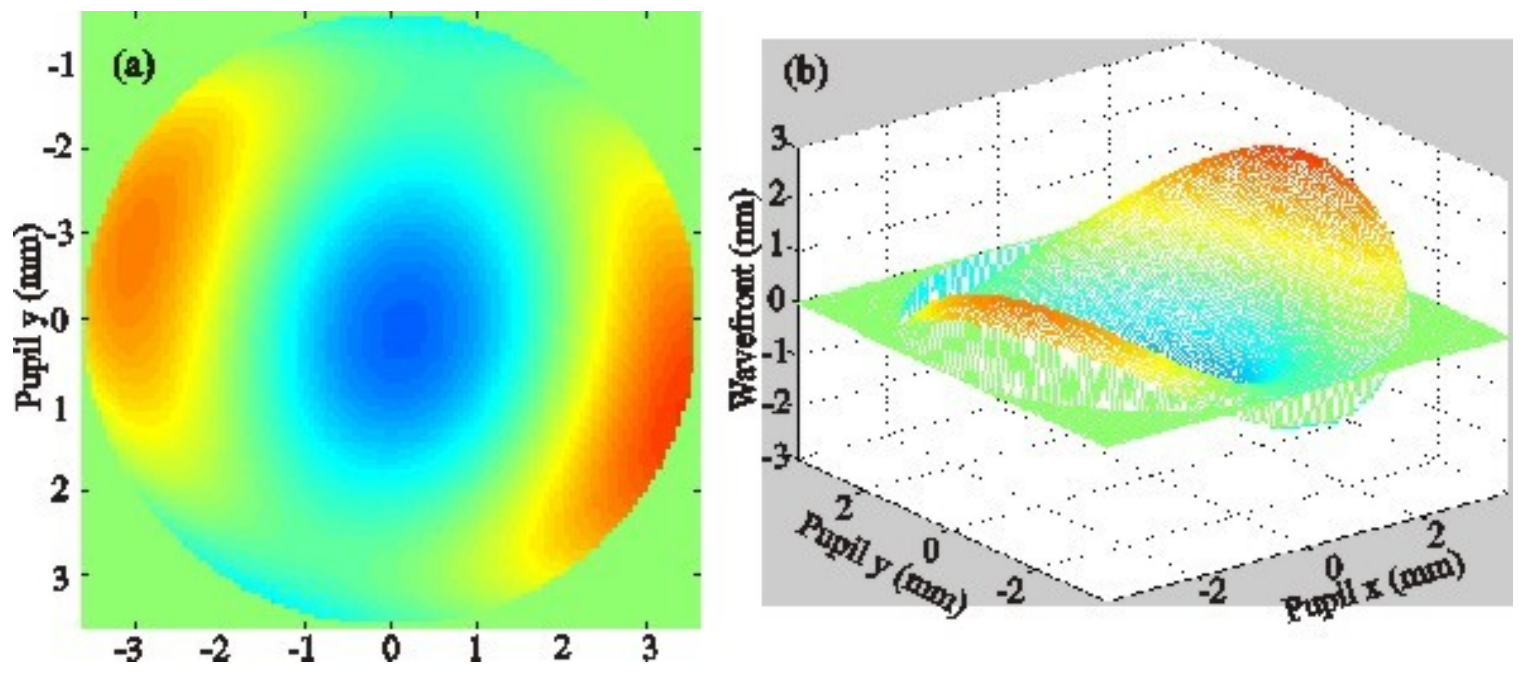

Figure 7.7: The uncorrected (flat DM) wavefront recorded from the Cain-cell lens in (a) 2D and (b) 3D, without thermal lensing (the NIR beam was blocked).

The corresponding PSF to the wavefront shown in Fig. 7.7 is shown in

Fig. 7.8.

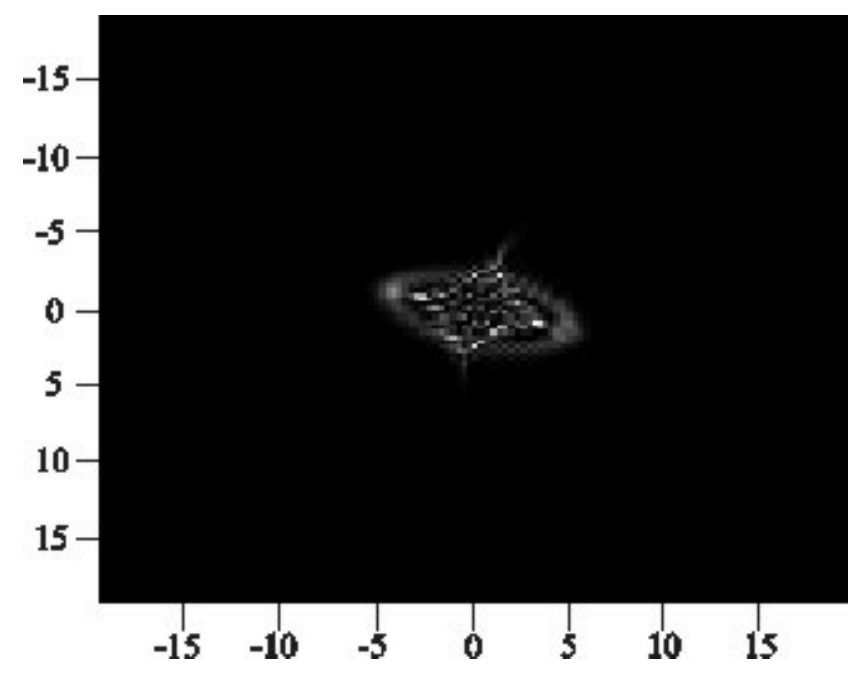

Figure 7.8: The PSF corresponding to the wavefront in Fig. 7.7.

Given the experimental procedure the aberrations induced by the thermal lens depicted in Fig. $7.5 \mathrm{c} \& \mathrm{~d}$ and Fig. 7.6b are due only to the thermal lens and not the Cain- 
cell. The significance of the aberrations for the uncorrected initial state Cain-cell is reserved for the discussion.

The five strongest Zernike polynomial coefficients, strength chosen based upon peak magnitude during the steady-state response during the thermal lensing exposure, were plotted as a function of time in Fig. 7.9. Each vector of coefficients was offset relative to their respective values at initial-state with the corrected wavefront (Fig. 7.5a \& $\mathrm{b}$ and Fig. 7.6a). A noise signal of $\sim 1.46 \mathrm{~Hz}$ was observed throughout the data set (meaning the noise was present regardless of the DM being on or off and regardless of the thermal lens). Using Matlab, a $5^{\text {th }}$-order lowpass Butterworth digital filter was applied to the vector of coefficient values with a $1.2-\mathrm{Hz}$ cut-off frequency in order to remove this noise source.

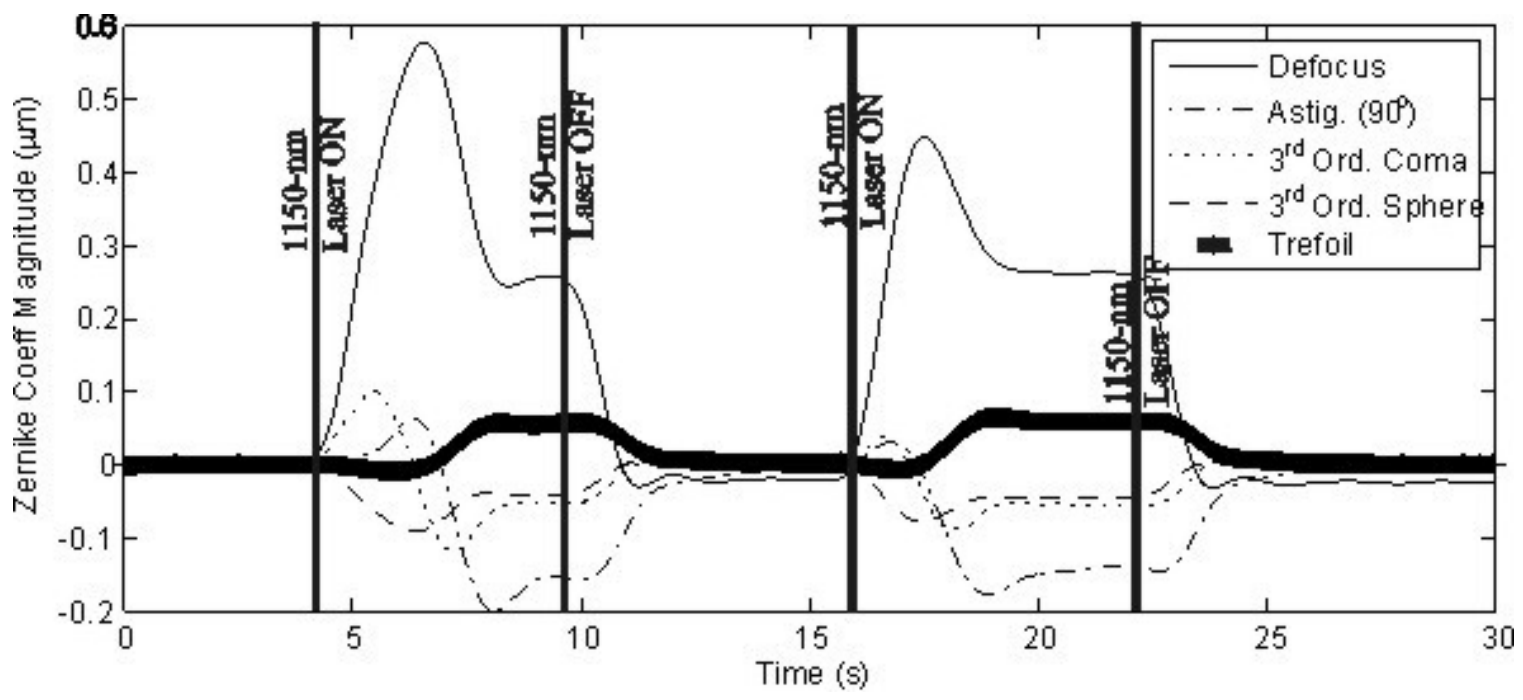

Figure 7.9: The transient response to the thermal lens for five Zernike polynomial coefficients. The $1150-\mathrm{nm}$ exposures were approximately 7.5 -s and 8.25 -s for the first and second exposures respectively. 


\subsection{DISCUSSION}

At first glance, the aberrations induced in the wavefront for the initial-state uncorrected (DM off) Cain-cell in Fig. 7.7 and 7.8 seem to suggest that the aberrations induced by the thermal lens in Fig. $7.5 \mathrm{c} \& \mathrm{~d}$ and Fig. $7.6 \mathrm{~b}$ are weaker than the natural state of the "eye." However, based on the appearance of the PSF in Fig. 7.8, the aberrations found in the Cain-cell lens at initial-state with a flat DM were significantly stronger than the average eye as reported in Thibos, Hong et al. 2002. The artificial retina placed into the Cain-cell at the focal plane was decoupled from the Cain-cell lens position and may not have been perfectly normal to the incoming wavefront. If the artificial retina was skewed from the optimal position of the Cain-cell lens, such as tilted and/or rotated, it may have contributed to these initial-state aberrations. Thus the aberrations shown for the Cain-cell at initial-state Fig. 7.7 and 7.8 should not be confused with the aberrations in a perfect eye.

Though there were strong aberrations present in the initial-state of the artificial eye (Fig. $7.7 \mathrm{a} \& \mathrm{~b}$ ) the Iris $\mathrm{AO}$ system was able to correct these initial-state aberrations (Fig. 7.5 a \& b), flattening the wavefront. This allowed for the aberrations induced by the thermal lens to be observed independently from the optical aberrations of the system (Fig. $7.5 \mathrm{c} \& \mathrm{~d})$. The Iris AO, Inc. system's software further provided the capability to observe the PSF (Fig. 7.6) correlating with each reconstructed wavefront.

A unique advantage of using an AO system to observe the thermal lens is the ability to track the Zernike polynomial coefficients as a function of time (rate of $30-\mathrm{Hz}$ ) (Fig. 7.9). This allowed for investigating the types of aberrations induced by the thermal lens in an artificial eye and could be extended into an animal model for an in vivo analysis. This analysis revealed that the thermal lens causes several aberrations with the 
Zernike polynomial (Table 7.1) associated with the defocus (mode 4) term being the strongest, followed by astigmatism ( 0 and $90^{\circ}$ axis) (mode 5$), 3^{\text {rd }}$ order horizontal coma (mode 8), $3^{\text {rd }}$ order spherical (mode 12) and trefoil (mode 9). The 6-10 strongest Zernike coefficients (at steady-state) were not presented, but rank in order of next strongest to weakest as astigmatism $\left(45^{\circ}\right.$ axis), $3^{\text {rd }}$ order vertical coma, $2^{\text {nd }}$ order astigmatism, quadrafoil, and trefoil which are associated with Zernike polynomial modes 3, 7, 13, 14, and 6 respectively.

In Fig. 7.9 it is interesting to note when higher-order aberrations appear in the thermal lens. Upon exposure to the CW 1150-nm source, the defocus term immediately begins to rise. The trefoil term appears to dip slightly at the start of the thermal lens, and then begins to rise $\sim 2.2 \mathrm{~s}$ and $\sim 1.8 \mathrm{~s}$ after the start of response from the defocus term in the first and second exposures respectively. The trefoil term appears to persist longer, after the 1150-nm laser source is turned off, compared to the defocus term. The other three aberration types (astigmatism, coma and spherical) depicted in Fig. 7.9 appear to respond immediately upon exposure to the 1150-nm laser radiation source without delay.

The defocus term in Fig. 7.9 rises steadily for $\sim 2.5$ and $\sim 1.8$ seconds for the first and second 1150-nm exposure respectively. After this time, each of the defocus terms drop to a knee until the laser source turns off. At this time, it is believed this knee shape arises due to small convectional flows occurring in the water sample caused by volumetric heating at steady-state.

The magnitude and transient response of each Zernike polynomial coefficient is expected to change according to the CW NIR wavelength, and beam-diameter and power delivered to the corneal plane. The strength and transient response of the aberrations induced in the artificial eye by the thermal lens is also expected to follow similar trends 
presented in Chapter 3. However, unlike the technique used in Chapter 3, the technique used in this chapter can be extended to analyze the thermal lens for an in vivo application. Measurements to obtain qualitative data on aberrations induced by thermal lensing for an in vivo experiment will be conducted by the Air Force Research Lab.

\subsection{CONCLUSION}

Adaptive optics is a developing technology typically used for correcting wavefront aberrations in optical imaging systems. This technology can be used to analyze a thermal lensing event in the eye allowing for analysis of individual aberration types. This analysis shows evidence that thermal lensing involves higher order aberrations, beyond the expected defocus and astigmatism, to include $3^{\text {rd }}$ order horizontal coma, $3^{\text {rd }}$ order spherical and trefoil aberration types. As long as exposure conditions are kept below damage thresholds, this technique could be used in an animal eye to investigate the thermal lens in vivo.

\subsection{ACKNOWLEDGEMENTS}

The author would like to thank Iris AO, Inc., Dr. Jeff Oliver of the Air Force Research Laboratory, and Mr. Gary Noojin from Northrop Grumman for their assistance with data in this chapter. 


\section{CHAPTER 8}

\section{CONCLUSION AND FUTURE STUDIES}

\subsection{SUMMARY AND CONCLUSION}

This research was conducted in relation to basic laser-tissue effects studied at the Air Force Research Laboratory's (AFRL) Human Effects, Optical-Radiation Branch. The efforts of these studies have lead to a better understanding of the laser-tissue interaction between continuous-wave, CW, near-infrared, NIR, laser radiation and ocular media. These data assist with the development of laser safety parameters for the ANSIZ136 series in addition to computational modeling testing and validation at AFRL.

Thermal lensing is a natural phenomena arising in a material, biological or otherwise, which absorbs laser radiation. The primary goals of this dissertation were to quantify to what extent thermal lensing occurs in ocular media and relate how the natural phenomenon impacts the retinal damage parameters. These goals were achieved using a variety of methods to include mathematical modeling, experimental observation and quantification, and establishing the CW NIR retinal damage threshold.

Laser radiation from NIR wavelengths is capable of inducing a thermal lens which defocuses a beam by several diopters in the eye depending upon the power and linear absorption coefficient of the laser's wavelength in the ocular media (Chapter 3). These data demonstrated the multidimensional dependency of the thermal lens in the eye in terms of power, time, and the linear absorption coefficient by wavelength. In Chapter 4, a mathematical model described the relationship of the thermal lens in a simple eye's

optical geometry, finding that the beam-waist radius could change by as much as a factor 
of 2 (see Chapter 4 Fig. 4.7) within the Chapter 5 ED $_{50}$ for 1319-nm (an 80-ms exposure at $14.5-\mathrm{W})$. Though all evidence indicates that the beam-waist radius is growing as a function of time due to the thermal lens, retinal damage is still possible from a 1319-nm, 80-ms laser radiation exposure.

Thermal lensing does not protect the retina unconditionally from weakly transmitted, highly absorbed CW NIR laser radiation wavelengths between 1150-1350$\mathrm{nm}$ as evident by the retinal-damage study results in Chapter 5. In fact, the data were found to scale remarkably well using the scaling factor (SF) method which neglects thermal lensing entirely. Thermal lensing is a natural phenomenon and occurs, to some extent, in an eye exposed to laser radiation. The SF method used to fit retinal threshold data in Chapter 5 may unknowingly account for some portions of thermal lensing in the algorithm used to predict chromatic dispersion for wavelengths in the NIR. However, the modeling study in Chapter 6, predicts that thermal lensing protects the retina from $\mathrm{CW}$ NIR damage for smaller beam-diameters $(<\sim 4 \mathrm{~mm})$ delivered to the corneal plane for wavelengths where less than $\sim 5 \%$ energy is transmitted to the retina. The algorithm to calculate the temperature rise in Chapter 6 was also found to be comparable to the temperature calculation by the AFRL BTEC model (for equivalent input conditions of optical and thermal coefficients and beam-waist radii for a given time step).

\subsection{FUTURE STUDIES}

\subsubsection{Retinal ED $_{50}$ as a Function of Delivered Corneal Spot-size}

The CW NIR retinal $\mathrm{ED}_{50}$ has not been determined in terms of delivered corneal spot-size. Currently, the AFRL does not possess the capability to reduce the spot-size of the 1319-nm laser-beam delivered to the corneal plane due to limitations of the laser's 
performance. The retinal $\mathrm{ED}_{50}$ could be determined for the 1110 -nm wavelength for a 3$\mathrm{mm}$ beam diameter delivered to the cornea. This would act as a control to establish the expected reduction in retinal $\mathrm{ED}_{50}$ for a smaller beam-diameter for CW NIR wavelengths. The same experiment could then be conducted for the 1319-nm wavelength to determine if the two wavelengths follow the same trend. If the trend between delivered corneal spot-size and retinal $\mathrm{ED}_{50}$ from 1319 -nm laser radiation were found to be linear, then this would suggest thermal lensing does not protect the retina. If the $1319-\mathrm{nm}$ retinal $\mathrm{ED}_{50}$ were determined to increase with decreasing corneal spot-size, then this would confirm the results found in Chapter 6 which predict that the thermal lens does afford some protection of the retina for specific conditions.

\subsubsection{Quantifying Aberrations Induced by Thermal Lensing in vivo}

The adaptive optics (AO) technique used in Chapter 7 should be used on a real primate eye. Given the limitation of the $30-\mathrm{Hz}$ operation of the AO system, it would be beneficial to reduce the power from the NIR laser source exposed to the eye, permitting a

longer exposure, but should be kept below retinal damage threshold. These data would provide valuable information on the aberration modes which arise from the thermal lens and could be analyzed to estimate the strength of the thermal lens in terms of defocus for a particular exposure time, power, wavelength and input beam diameter.

\subsubsection{Histology and Advanced Imaging}

Chapter 2 described how Zuclich, Lund et al. 2004 determined that threshold NIR lesions appeared larger than visible wavelength lesions and involved the full-retinal thickness. Damage involving the full-retinal thickness is a trait normally only seen at visible wavelengths for suprathreshold exposures, exposures which are greater than the $\mathrm{ED}_{50}$ threshold. Zuclich, Lund et al. 2004 also reported subthreshold NIR exposures 
which resulted in lesions that were only visible by a laser-scanning ophthalmoscope (LSO). These subthreshold lesions were verified by histology, but because they were not visible by conventional fundus camera observation, they are not considered to be a true retinal lesion. The endpoint in determining a retinal lesion for a conventional $\mathrm{ED}_{50}$ study is by visual observation of the anterior retina through a fundus camera, but it may be that lesions are present which do not appear in this conventional method. Histology and advanced imaging modalities such as optical coherence tomography (OCT) and linescanning laser ophthalmoscope (LSLO) would assist in determining if subthreshold lesions are present and persistent in deeper layers in the retina.

\subsubsection{Advanced Retinal Damage Modeling}

The model used in Chapter 6 is based upon a straight forward ABCD beam propagation algorithm with the retina represented as a simple homogenous slab. The $\mathrm{ABCD}$ beam propagation method should not be used to estimate beam geometries in highly scattering tissues, where the laser-beam's profile begins to vary significantly with depth into the tissue. Given the complexity of the tissues composing the retina, a more advanced retina model would be beneficial to provide insight about the origin of retinal damage (more posterior or anterior), but requires a more complex mathematical model due to the scattering properties associated with the retinal tissue. Advanced models of thermal lensing through the eye are being developed at AFRL's Optical Radiation Branch which incorporate optical scattering, photon distributions in tissue, axial thermal diffusion and finite element analysis. These higher fidelity mathematical models go hand-in-hand with a histological study of the damage from CW NIR laser radiation and studies determining the optical properties of tissues. 


\subsubsection{Measuring Thermal and Optical Properties of Tissues}

Full-animal models are costly and are inherently difficult to isolate specific properties of a tissue without invasive measures. In vitro experimental models have been an increasing interest in laser-tissue research (Denton, Foltz et al. 2007; Denton, Foltz et al. 2008; Denton, Foltz et al. 2009). These models use cell cultures designed to simulate a target tissue in the body. Studies by Denton, Foltz et al. 2007-2009 investigating the role of melanin in laser-tissue interaction use extracted melanin granules from the retinas of bovine eyes which are fed to a live cell culture. The cells ingest the melanin granules, causing them to optically mimic the pigment epithelium layer of the retina. Cell cultures can then be selectively exposed to laser radiation, then fluorescently labeled to search for cell death using fluorescence microscopy. Denton, Foltz et al. 2009 have recently reported using a microthermograpy technique which uses an infrared camera to record the temperature profiles on the surface of the cell cultures during laser exposure, allowing for temperature distributions to be compared to fluorescence microscopy results correlating with cell death.

Microthermography in vitro studies of pigment epithelium will provide an excellent analysis to determine absorptive properties of PE and thermal properties as a function of wavelength in the NIR. These studies will determine the magnitude of the absorption coefficient and thermal conductivity of PE to be used in advanced laser-tissue damage models of the retina. 


\section{Appendix A}

\section{DOCUMENTATION OF CODE FOR CHAPTER 4}

\section{Definition of Variables: \\ User Input Variables}

lambda $=$ Wavelength $(\mathrm{m})$

$\mathrm{P}=$ Power $(\mathrm{W})$ reaching the sample

$\mathrm{n} \_$air $=$Index of refraction of air for the wavelength being tested

$\mathrm{dz}=$ Step size to take through sample $(\mathrm{m})$

$\mathrm{dt}=$ Initial time step to take (s)

dn_dT $=$ Thermo-Optic Coefficient $\left(\mathrm{K}^{-1}\right)$

Thick $=$ Sample path-length

total $\mathrm{t}=$ Total time the sample is exposed to the radiation source

$\mathrm{T}=$ Initial temperature of the sample, taken to be room temperature $(\mathrm{K})$

$\mathrm{d}=1 / \mathrm{e}^{2}$ beam-diameter delivered to the front-face of the focusing lens $(\mathrm{m})$

$\mathrm{f}=$ Focal length of the focusing lens $(\mathrm{m})$

z_positions_of_sample = Array of values containing the z-positions of the front-face of the sample cuvette relative to the initial focus of the focusing lens $(\mathrm{m})$

far_field $=$ Distance from the focal plane to the far-field plane $(\mathrm{m})$

aperture radius $=$ Radius of the aperture placed at the far-field $(\mathrm{m})$

z_scan_data $=$ Normalized z-scan data array containing the z-positions of the sample in the first column, and the normalized irradiance at the end of the exposure time for each z-position of the sample in the second column

\section{Remaining Variables:}

eta $=\eta$, Thermal Diffusivity of sample $\left(\mathrm{m}^{2} / \mathrm{s}\right)$

kappa $=\kappa$, Thermal Conductivity of sample $(\mathrm{W} /(\mathrm{m} * \mathrm{~K}))$

rho $=\rho$, Density of sample $\left(\mathrm{kg} / \mathrm{m}^{3}\right)$

$\mathrm{cp}=\mathrm{c}_{\mathrm{p}}$, Specific Heat at Constant Pressure $(\mathrm{J} /(\mathrm{kg} * \mathrm{~K}))$

muaw $=\mu_{\mathrm{a}}$, Linear Absorption Coefficient of Water $\left(\mathrm{m}^{-1}\right)$

$\mathrm{n}=$ Initial/background index of refraction of the sample

$\mathrm{q}=$ Complex Beam Parameter

$\mathrm{W}=\omega$, the $1 / \mathrm{e}^{2}$ beam-waist radius which is a function of time $(\mathrm{m})$

$\mathrm{t}=$ time $(\mathrm{s})$

$\% \% \% \% \% \% \% \% \% \% \%$ START MAIN PROGRAM for Z-Scan $\% \% \% \% \% \% \% \% \% \% \% \%$

$\% \% \% \% \% \% \% \% \% \% \% \% \% \% \quad$ TO BE RUN IN MATLAB $\quad \% \% \% \% \% \% \% \% \% \% \% \% \% \%$

$\%$ This program was created to simulate the original z-scan data from

\%December of 2005 (published in SPIE 2006) for a long focal length "thin" lens

\%Outputs: 
$\%$ z_scan_data $=2 \mathrm{D}$ array containing the classic normalized z-scan data array containing the z$\%$ positions of the sample in the first column, and the normalized irradiance at the end of the $\%$ exposure time for each z-position of the sample in the second column.

$\%$ w_t_z_normalized $=2 \mathrm{D}$ array z-positions of the sample go across in the cols while the time steps go down in the rows.

\%This code calls the sub-functions:

$\%$ Z_Scan_Parameters.m

\%ThermalLens_Basic_Z_Scan.m

\%Created by Rebecca Vincelette

format compact;

clear all; close all; clc;

z_positions_of_sample $=[-0.305: 0.01: 0.305] ; \%(\mathrm{~m})$

far_field $=0.7 \overline{7} ; \%(\mathrm{~m})$

aperture_radius $=1.25 * 10^{\wedge}-3 ; \%(\mathrm{~m})$

$\%$ Get variables

$\mathrm{y}=\mathrm{Z} \_$Scan_Parameters;

$\mathrm{P}=\mathrm{y}(1)$

lambda $=\mathrm{y}(2)$;

$\mathrm{n}$ _air $=\mathrm{y}(3)$;

eta $=y(4)$

kappa $=\mathrm{y}(5)$;

$\mathrm{dn} \_\mathrm{dT}=\mathrm{y}(6)$;

$\mathrm{dt}=\mathrm{y}(7)$;

total_t $=y(8)$;

total_length $=\mathrm{y}(9)$;

$\mathrm{dz}=\mathrm{y}(10)$;

mua $=\mathrm{y}(11)$;

$\mathrm{f}=\mathrm{y}(13)$;

$\mathrm{d}=\mathrm{y}(14)$;

$\mathrm{n}=\mathrm{y}(15)$;

Sample_Thickness $=\mathrm{y}(16)$;

\%Initialize variables

$\mathrm{z}=$ zeros((length(z_positions_of_sample)),2500);

$\mathrm{w}=\mathrm{z}$;

$\%$ Now consider thermal lensing effect applied to the results from $\mathrm{ABCD}$

$\%$ Propagation

for index $=1$ :length(z_positions_of_sample),

evaluating $z=z$ positions_of_sample(index)

position_of_sample $=\mathrm{z}$ _positions_of_sample(index);

[output, w_transient, $\mathrm{t}]=$ ThermalLens_Basic_Z_Scan(lambda, P, position_of_sample, far_field,sample, 'y', transient); 
[reference, not_important, not_imp] $=$ ThermalLens_Basic_Z_Scan(lambda, P, position_of_sample, far_field,sample, ' $n$ ', ' $n$ ');

temp1 $=$ output $(1,:)$;

temp2 = output $(2,:)$;

temp3 = output $(3,:)$;

$\mathrm{z}($ index, $1:$ length $($ temp 1$))=$ temp 1 ;

$\mathrm{w}($ index, 1 :length $($ temp2) $)=$ temp2; \% This should be a 2-D matrix containing the beam waist

values over all z-space

$\%$ for all z-positions of the sample. Z-positions of the sample are

$\%$ represented going down the rows of this matrix.

get_last_w_infarfield $=$ find $($ temp $2 \sim=0)$;

temp4 = temp2(get last_w_infarfield);

far_field_w(index $)=$ temp $\overline{4}$ (end); \% Want to save the beam-waist radius calculated at the farfield plane

w_t_z(:,index $)=$ w_transient'; \%z-positions of the sample go across in the cols while the

time steps go down in the rows

clear output; clear w_transient; clear not_important; clear not_imp;

end

ratio $=((2 *$ aperture_radius $) . /(2 . *$ far_field_w $)) \cdot \wedge 2$;

w_output $=(1-\exp (-($ ratio $)))$;

refw_output $=$ w_output $(1) ; \%$ This is the reference for which data will be normalized

w_output $=($ w_output./refw_output $) ; \%$ This normalizes the data to the reference

tempy $=\left((2 *\right.$ aperture_radius $\left.) \cdot /\left(2 \cdot{ }^{*}{ }_{\mathrm{w} \_} \mathrm{t} \_\mathrm{z}\right)\right) \cdot{ }^{\wedge} 2$;

w_t_z_normalized $=\overline{(1-\exp (-(\text { tempy }))})$;

vector $=\mathrm{t}$;

for count $=1$ :length(vector), $\%$ Execute for the length of the time vector

tempx $=$ w_t_z_normalized(count, : );

refx $=$ tempx $(1,1)$;

w_t_z_normalized(count, :) = tempx./refx;

end

z_scan_data $(:, 1)=$ z_positions_of_sample';

Z_scan_data $(:, 2)=$ w_output';

\% $\% \% \% \% \% \% \% \% \% \% \%$ END MAIN PROGRAM for Z-Scan $\% \% \% \% \% \% \% \% \% \% \% \% \% \%$

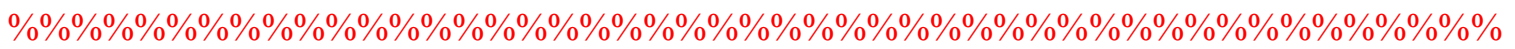

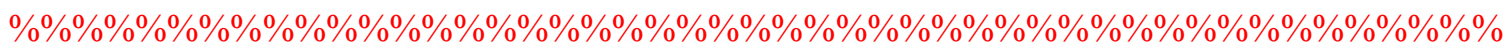

$\% \% \% \% \% \%$ START SUBFUNCTION Z_Scan_Parameters.m \% \% \% \% \% \% \% \% \% \% \% \% \% $\%$

function $Y=$ Z_Scan_Parameters;

$\%$ This function is intended to be used as the file to make changes to

\%user input variables.

$\%$ This function calls the sub-functions 


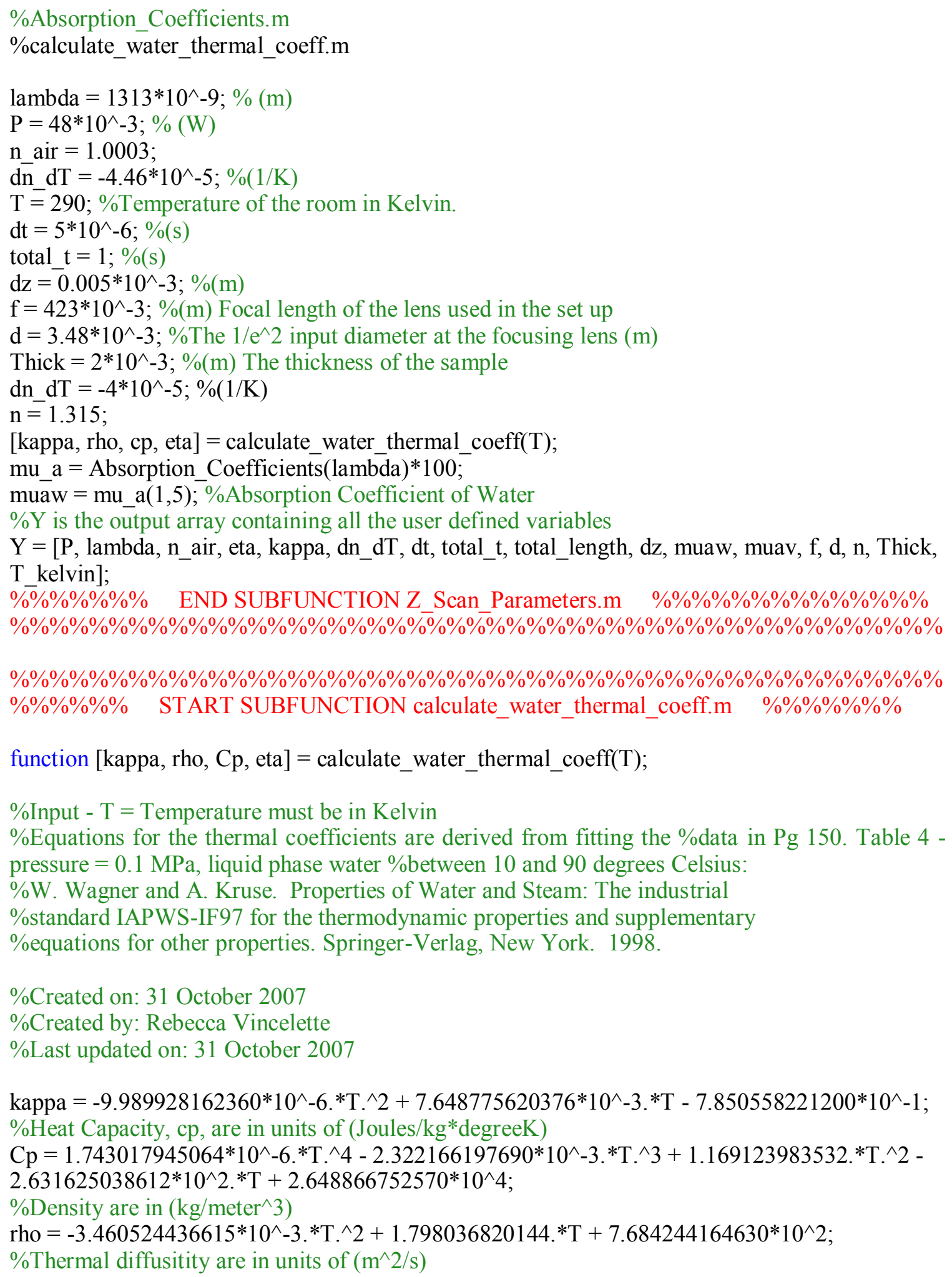


eta $=$ kappa. $/\left(\right.$ rho.$\left.{ }^{*} \mathrm{Cp}\right)$;

$\% \% \% \% \% \%$ END SUBFUNCTION calculate water thermal coeff.m $\% \% \% \% \% \% \% \%$

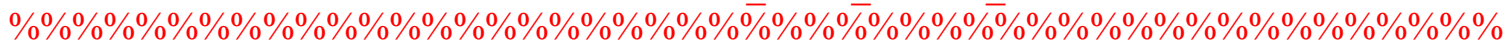

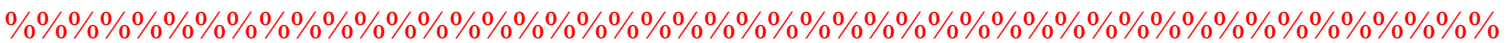

$\% \% \% \% \% \%$ START SUBFUNCTION Absorption_Coefficients.m \% \%\%\%\%\%\%\%\%\%\%\%\%

function output $=$ Absorption_Coefficients(lambda_o $)$;

$\%$ lambda_o = the input wavelength (m)

$\%$ This function uses a piece-wise function algorithm to fit to the Maher

$\%$ absorption data for Rhesus monkey eyes. Where piece-wise was too

$\%$ difficult, linterp functions were used on the Maher data.

\% The input, lambda_o, must be in $\mathrm{m}$.

$\%$ The piece wise functions are valid from 400-1340 nm for cornea, \%aqueous, humor, lens and vitreous humor and water in $1 \mathrm{~nm}$ increments.

$\%$ Output $=$ [muac, muaa, mual, muav, muaw $]$

$\%$ E. F. Maher, "Transmission and absorption coefficients for the ocular $\%$ media of the Rhesus

monkey," in SAM -TR-78-32, pp. 1-104, USAF School \% of Aerospace Medicine (1978)

$\%$ Hale and Querry, "Optical constants of water in the 200-nm to $200-\mathrm{mm} \%$ region," Applied Optics, 12(3). pp. 555-563, (1973).

\%Last modified on: November 26, 2007

$\%$ Created on: September 5, 2007

$\%$ Created by: Rebecca Vincelette

lambda_o $=$ round(lambda_o*10^9); \%Convert to $\mathrm{nm}$

data $=\overline{\text { load}}($ 'absorption_coeff.txt');

$\%$ The units of the text file loaded here are nm on wavelength and $1 / \mathrm{m} \%$ for the absorption

coefficients

lambda $=$ data $(:, 1)$;

muac $=$ data $(:, 2) ; \%$ Absorption coefficient for cornea

muaa $=$ data $(:, 3) ; \%$ Absorption coefficient for aqueous

mual $=$ data $(:, 4) ; \%$ Absorption coefficient for lens

muav $=$ data $(:, 5) ; \%$ Absorption coefficient for vitreous

muaw $=$ data(:,6); \%Absorption coefficient for water

index $=$ find $($ lambda $==$ lambda_o $)$;

output $=[$ muac(index $)$, muaa(index), mual(index), muav(index), muaw(index) $]$;

$\% \% \% \% \% \%$ END SUBFUNCTION Absorption_Coefficients.m $\quad \% \% \% \% \% \% \% \% \% \% \%$

\%\%\%\%\%\%\%\%\%\%\%\%\%\%\%\%\%\%\%\%\%\%\%\%\%\%\%\%\%\%\%\%\%\%\%\%\%\%\%\%\%\%\%\%\%\%\%

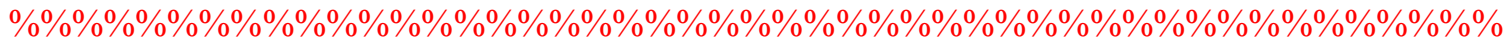
$\% \% \% \% \% \%$ START SUB-FUNCTION ThermalLens_Basic_Z_Scan.m \%\%\%\%\%\%\%\%\%\%

function [wzT, w_transient, time_vector] $=$ ThermalLens_Basic_Z_Scan(lambda_0, P, sam_pos, far_field, no_sample, thermal_lens, transient_analysis); 
\% This function evaluates an ABCD model for z-scan for a given \%wavelength.

$\%$ The user must describe the input parameters in a file \%z_scan_parameters.m

\%no sample - Make this value 'no' if you want to just check case for no

$\%$ sample in the beam path and just check the beam waist traversing \%through the z-scan optical configuration

\%transient_analysis - should be 'y' if you want to check the time \%response of the beam in the far-field

$\%$ This function calls the functions:

$\%$ ABCD.m

$\%$ waist.m

\%beamparameter_q.m

$\%$ calculate water_dndT.m

$\%$ get time.m

$\%$ Calculate_Temperature_Rise.m

\%ThermalLens_through_Z_ZSCan_sample.m

\%Last updated on: 13 Feb., 2008 - Updated the calculation of the temperature rise (T_vector)

$\%$ Created on: 15 Oct., 2007

$\%$ This is the 4th Generation of code - this one came from

$\%$ ThermalLens_Reduced_Chromatic_eye.m which was the 3rd generation of code

$\% 2$ nd Generation of code: Originally created from Chromatic_eye.m which was created on 27

Sept 2006

\%and ThermalLens simulated eye.m which was created on 7 Sept 2006

$\%$ Created by Rebecca Vincelette

$\%$ Get the input parameters for the z-scan

account_for_cell_wall = 'y'; \%Set this to 'y' if you want to account for the deformation of q from the cuvette walls

$\mathrm{y}=\mathrm{Z} \_$Scan_Parameters;

Po $=\mathrm{y}(1)$

lambda $0=\mathrm{y}(2)$;

$\mathrm{n} \_$air $=\mathrm{y}(3)$;

eta $=y(4)$

kappa $=\mathrm{y}(5)$;

$\mathrm{dn} \_\mathrm{dT}=\mathrm{y}(6)$;

$\mathrm{dt}=\mathrm{y}(7)$;

total_t $=y(8)$;

total_length $=\mathrm{y}(9)$;

$\mathrm{dz}=\mathrm{y}(10)$;

mua $=\mathrm{y}(11)$;

$\mathrm{f}=\mathrm{y}(13)$;

$\mathrm{d}=\mathrm{y}(14)$;

$\mathrm{n}=\mathrm{y}(15)$;

thick $=\mathrm{y}(16)$;

$\mathrm{T}=\mathrm{y}(17)$;

$\mathrm{t}=0 ; \%$ Initialize the time 


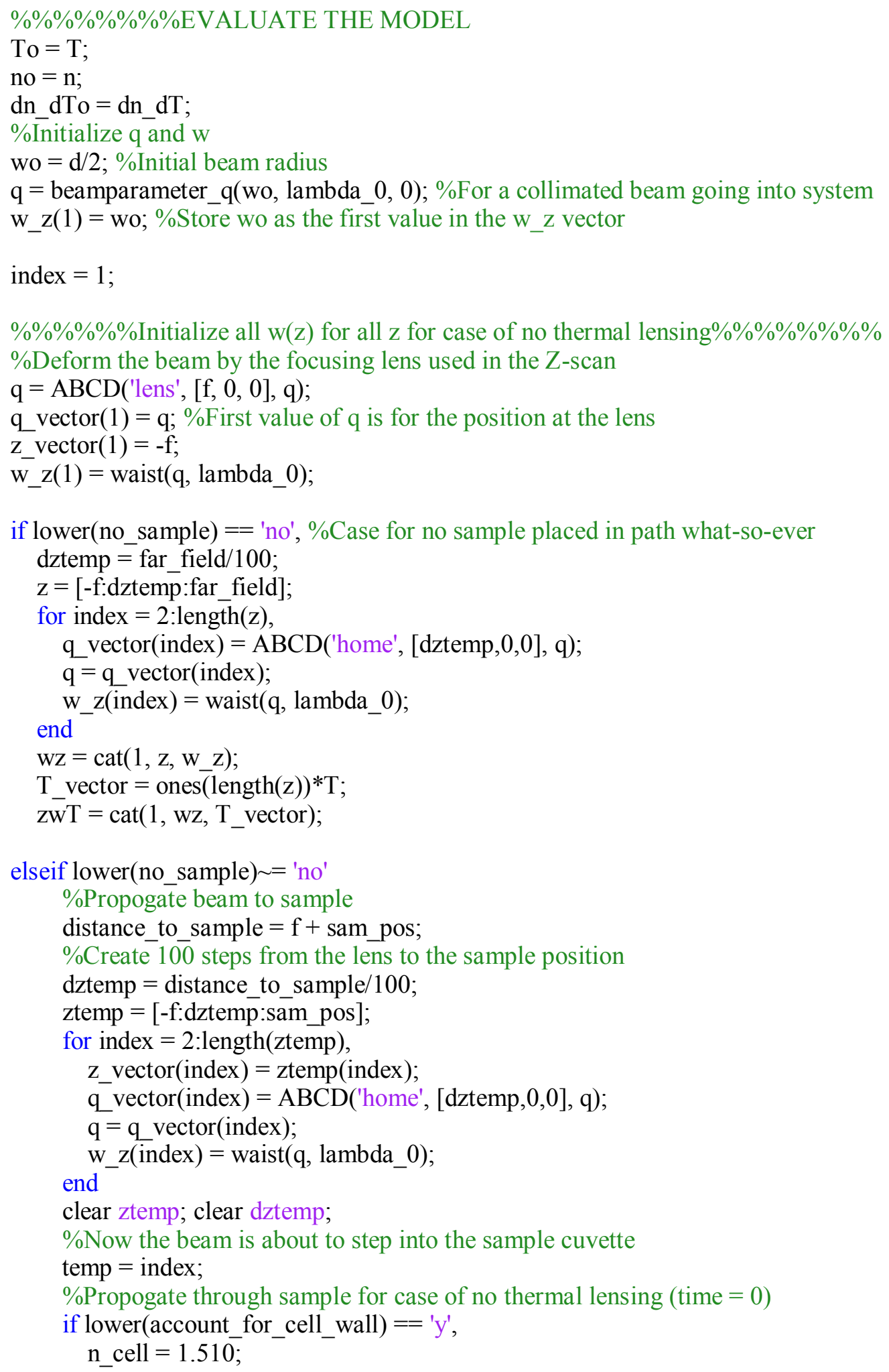


cell_wall_thickness $=1.25^{*} 10^{\wedge}-3 ; \%$ This is the thickness of the cuvettes wall

$\mathrm{q}=\overline{\mathrm{ABCD}}($ 'diin', [n_air, n_cell, 0], q);

$\mathrm{q}=\mathrm{ABCD}($ 'home', [cell_wall_thickness, 0,0$], \mathrm{q})$;

$\mathrm{q}=\mathrm{ABCD}($ 'diin', [n_cell, $\mathrm{n}, \overline{0}], \mathrm{q})$;

else

$\mathrm{q}=\mathrm{ABCD}($ 'diin', [n_air, $\mathrm{n}, 0], \mathrm{q})$;

end

$\mathrm{q}$ vector(index $)=\mathrm{q} ; \%$ Value of $\mathrm{q}$ for the position at the front face of the sample seed_q = q;

\%REMEMBER to update the wavelength everytime you move into a new boundary!

lambda1 = lambda_0 * (n_air/n);

w_z(index) $=$ waist $(\mathrm{q}$, lambda 1$)$;

index_sample_front $=$ index;

$\% \%$ START THERMAL LENS PREDICTION HERE

ztemp $=\left[\mathrm{sam} \_\right.$pos $+\mathrm{dz}: \mathrm{dz}: \mathrm{sam} \_$pos + thick $]$;

$\mathrm{z}=$ sam_pos;

index_front $=$ temp;

index_back = length(ztemp)+temp-1;

clear temp;

for index $=$ index_front:index_back,

if $\mathrm{z}<$ sam_pos + thick,

$\mathrm{z}=\mathrm{z}+\overline{\mathrm{d} z}$

q_vector(index $)=\operatorname{ABCD}($ 'home', $[\mathrm{dz}, 0,0], \mathrm{q})$;

$\mathrm{q}=\mathrm{q}$ vector(index);

w_z(index) = waist $(\mathrm{q}$, lambda 1$)$;

z_vector(index) $=\mathrm{z}$;

end

end

$\%$ I think the segment to get out of the sample should be

$\%$ after the thermal lens segment so the iterative portion of the code only

$\%$ converges on a solution to the beam waist in the sample where thermal

$\%$ lensing is taking place. This saves on computational time.

$\mathrm{w} \_$new $=\mathrm{w} \_\mathrm{z}$ (index_front:index_back);

T_end_index $=$ index_back-index_front +1 ;

T_vector(1:T_end_index $)=$ To;

$\mathrm{w} \_$previous_waist $=\mathrm{w} \_$new;

if

\%Initialize the vectors through the sample

$\mathrm{dn} \_\mathrm{dT}=$ ones $\left(1, \mathrm{~T} \_\right.$end_index $) * \mathrm{dn} \_\mathrm{dTo}$;

$\mathrm{n}=$ ones $(1, \mathrm{~T}$ _end_index $) *$ no;

lambda1 $=$ ones $(1, \mathrm{~T}$ _end_index $) *$ lambda 1 ;

time index $=0$;

while $\mathrm{t}<$ total_t; \%Begin time loop

time index $=$ time_index $+1 ; \%$ Upate time index

q_old $=$ q_vector(index_sample_front);

time_info $=$ get_time $(\mathrm{dt}, \mathrm{t}$, total_t $\mathrm{t})$;

$\mathrm{t}=$ time_info $(1, \overline{1}) ; \mathrm{dt} 2=$ time_info $(1,2)$;

$\%$ Create q_old and w_z_old 
W_z_old $=$ w_new;

output $=$ ThermalLens_through_Z_Scan_sample(lambda_0, w_z_old, seed_q, lambda1,

Po, t, dn_dT, T_vector, $\mathrm{n}$ );

clear w_new;

$\mathrm{w} \_$new $=\operatorname{output}(1,:) ; \mathrm{q} \_$new $=\operatorname{output}(2,:)$;

T_vector);

delta_T_temp $=$ Calculate_Temperature_Rise(w_new, w_z_old, dt2, 0, Po, lambda_0,

T_vector $=$ T_vector + delta_T_temp;

delta_T $=\mathrm{T}$ _vector - To;

$\%$ Now re-calculate the dndT value based on the temperature

$\mathrm{dn} \_\mathrm{dT}=$ calculate_water_dndT(T_vector);

de $\bar{t}$ ta $\_n=d n \_d T . * \bar{d}$ elta_ $\overline{\mathrm{T}}$;

$\mathrm{n}=\overline{\mathrm{no}}+$ delta $\mathrm{n}$;

lambda $1=$ lambda_0.*(n_air./n);

w_t $(\text { time_index, }:)^{-}=\mathrm{w}_{-}$new; $\%$ This contains all beam waist values through the sample at each time step

\%Add the w_z value at that time step to the array containing all w_z values where each row represents a time step, starting with $t=0$ for the first row (case with no thermal lens)

$\% \mathrm{w} z$ z_array(time_index,: $)=\mathrm{w} \_$new; $\%$ Store the vector $\mathrm{w}(\mathrm{z})$ into an array to track over all space and time!

time_vector(time_index $)=\mathrm{t}$; \% Really, this is the time vector, not array

$\mathrm{q}=\overline{\mathrm{q}}$ new(end);

q_time_vector(time_index $)=\mathrm{q} \_$new $(\mathrm{end})$;

end $\%$ End time loop

$\mathrm{n}=\mathrm{n}$ (end) $\%$ Get the last value of $\mathrm{n}$ in the vector since this is at the back of the sample

$\mathrm{w} z \mathrm{z}$ (index_front:index back $)=\mathrm{w}$ new;

q_vector(index_front:index_back) $=\mathrm{q}$ _new;

temp $=\operatorname{size}\left(\mathrm{w}_{-} \overline{\mathrm{t}}\right) ;$ row $=\operatorname{temp}(1) ; \operatorname{col}=\operatorname{temp}(2)$;

$\mathrm{w} \_\mathrm{t} \_$end $=\mathrm{w} \_\mathrm{t}(:, \mathrm{col}-1)$;

if lower(transient analysis) $==$ ' $y$ ',

$\mathrm{z}$ jump $=$ far_field $-($ sam_pos + thick $)$;

\%tellme_where_Iam_jumping $=\mathrm{z}$ jump + sam_pos + thick;

for countx $=1:$ length(time_vector),

$\mathrm{q}$ old $=\mathrm{q}$ _time_vector $(\operatorname{count} \mathrm{x})$;

if lower(account_for_cell_wall) == 'y',

$\mathrm{qx}=\mathrm{ABCD}\left(\right.$ 'diin', $\left[\mathrm{n}, \overline{\mathrm{n}} \_\right.$cell, 0$], \mathrm{q}$ old $) ; \%$ Water to cuvette wall interface

$\mathrm{qx}=\mathrm{ABCD}($ 'home', [cell_wall_thickness,0,0], qx); \%deform the beam by the

distance of the cuvette wall

$\mathrm{qx}=\mathrm{ABCD}\left(\right.$ 'diin', [n_cell, $\mathrm{n} \_$air, 0$\left.], \mathrm{qx}\right) ; \%$ The cuvette wall to air interface

q time_vector(count $\bar{x})=\overline{A B C D}\left(\right.$ 'home', $\left[\mathrm{z} \_\right.$jump, 0,0$\left.], q x\right) ; \%$ This is the q-value all the way at the far-field position over all time

else

$\mathrm{qx}=\mathrm{ABCD}\left({ }^{\prime} d i i n ',\left[\mathrm{n}, \mathrm{n} \_\right.\right.$air, 0], qx);

q_time_vector(countx) $=\mathrm{ABCD}($ 'home', [z_jump, 0,0$], \mathrm{qx}) ; \%$ This is the q-value all the way at the far-field position over all time

end

end

end 


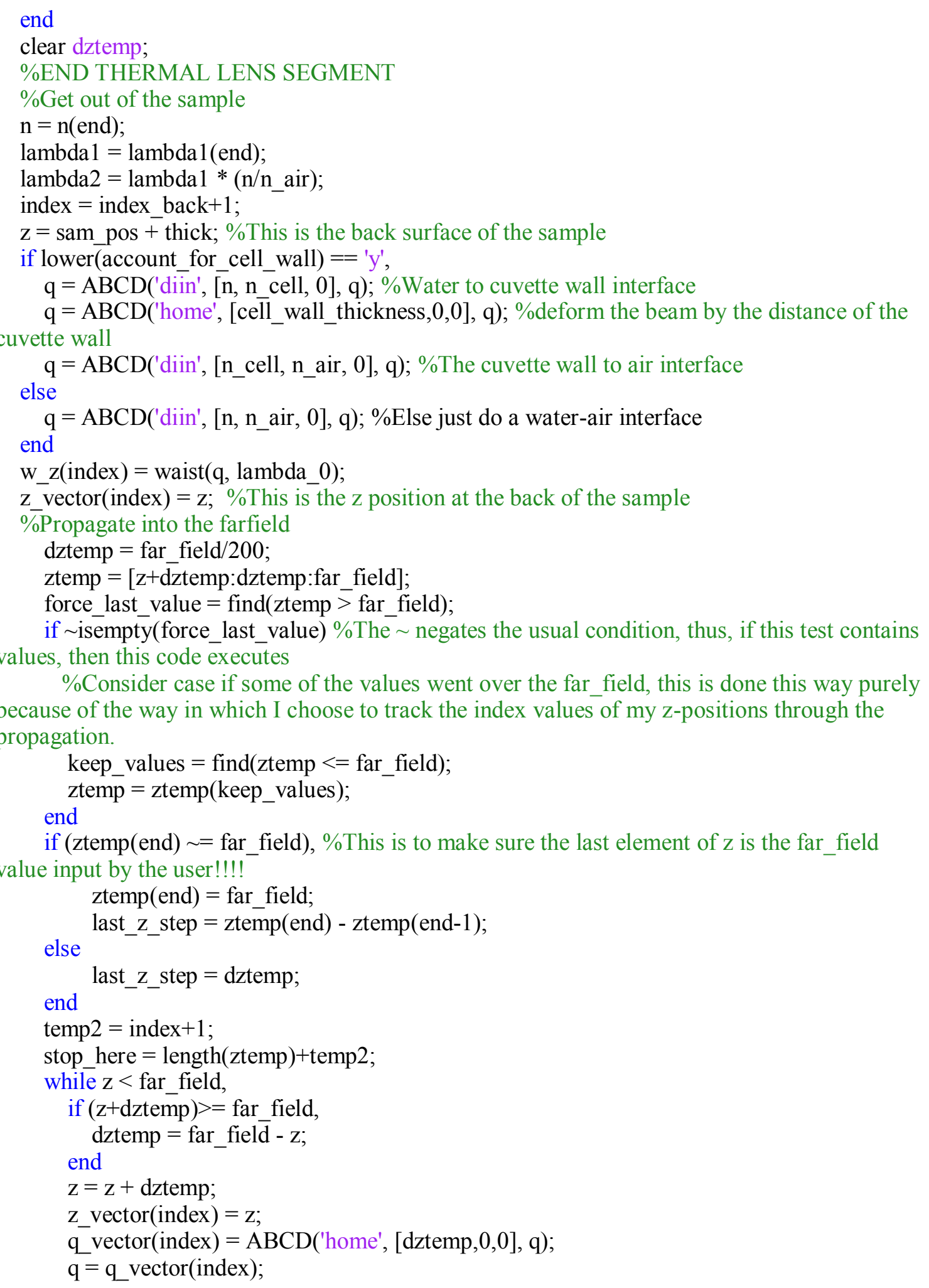




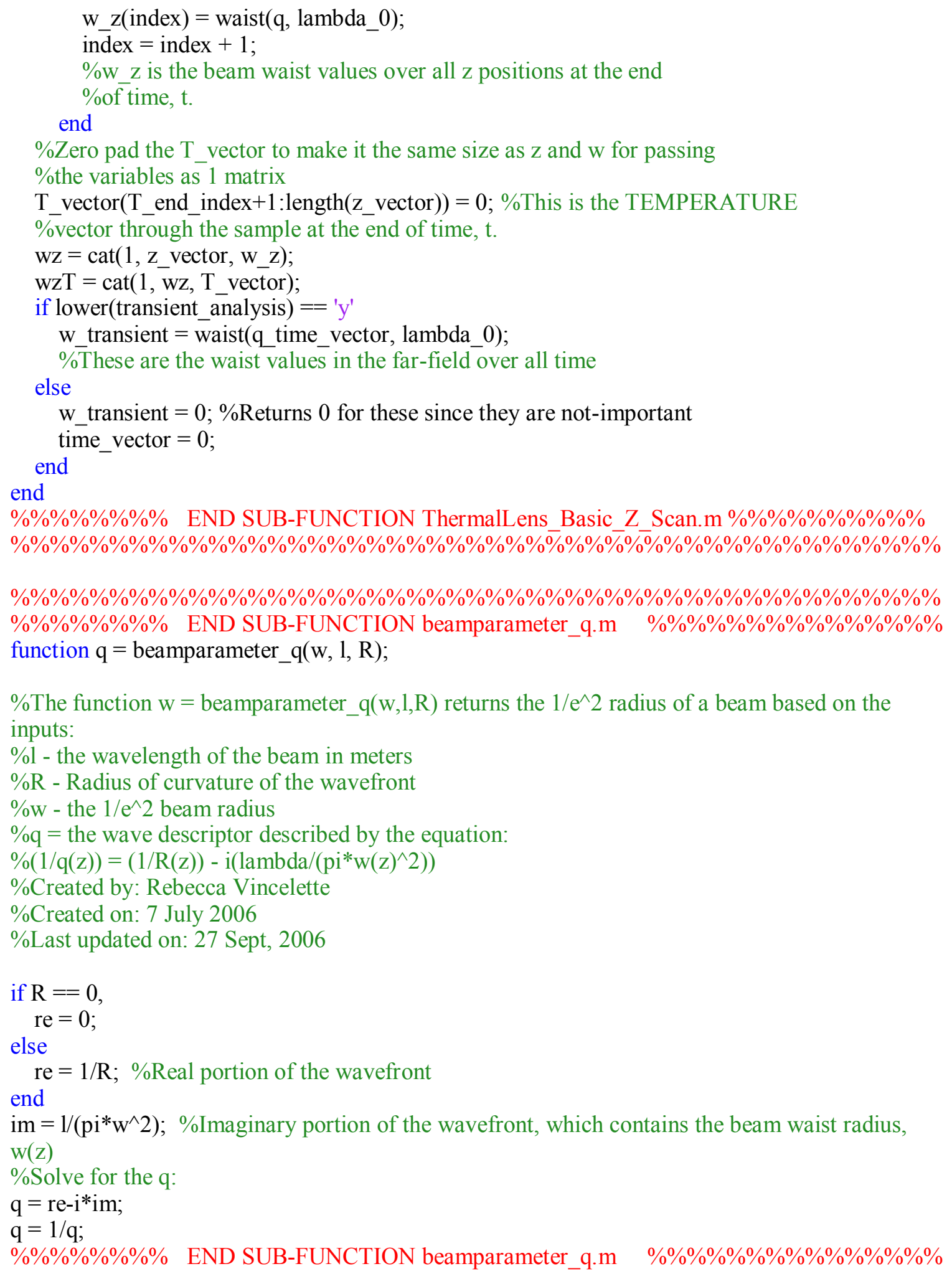




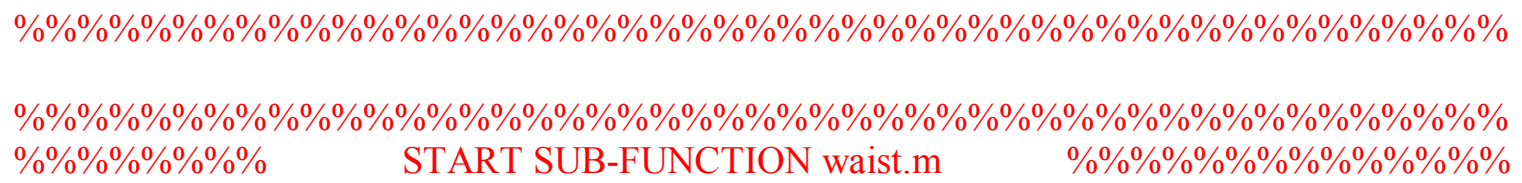


$\%$ Let $\mathrm{X}$ be a input matrix containing info about the indices of refraction, radius of curvature or length of medium as necessary

$$
\begin{aligned}
& \mathrm{x}=X(1,1) \\
& \mathrm{y}=\mathrm{X}(1,2) ; \\
& \mathrm{z}=\mathrm{X}(1,3) ; \\
& \mathrm{n} \text { _air }=1.0 ; \\
& \text { if type }==\text { 'lens' \%Thin Lens (where input } \mathrm{X}=[\mathrm{f}, 0,0] \text { ) } \\
& \quad \mathrm{f}=\mathrm{x} ; \\
& \quad \mathrm{a}=1 ; \\
& \quad \mathrm{b}=0 ; \\
& \quad \mathrm{c}=-1 / \mathrm{f} \\
& \mathrm{d}=1
\end{aligned}
$$

elseif type $==$ 'home' \%Homogenous Medium with length (thickness), $\mathrm{d}$ (where input $\mathrm{X}=[\mathrm{d}, 0$, $0]$ )

$$
\begin{aligned}
& \mathrm{d}=\mathrm{x} \\
& \mathrm{a}=1 \\
& \mathrm{~b}=\mathrm{d} \\
& \mathrm{c}=0 \\
& \mathrm{~d}=1
\end{aligned}
$$

elseif type $==$ 'diin' \%Dielectric Interface with refractive indices $n 1, n 2$ (where input $X=[n 1, n 2$, 0])

$$
\begin{aligned}
& \mathrm{n} 1=\mathrm{x} \\
& \mathrm{n} 2=\mathrm{y} \\
& \mathrm{a}=1 ; \\
& \mathrm{b}=0 ; \\
& \mathrm{c}=0 ; \\
& \mathrm{d}=\mathrm{n} 1 / \mathrm{n} 2
\end{aligned}
$$

elseif type == 'spdi' \%Spherical Dielectric with refractive indices $\mathrm{n} 1, \mathrm{n} 2$ and radius, $\mathrm{R}$ (where input $\mathrm{X}=[\mathrm{n} 1, \mathrm{n} 2, \mathrm{R}])$

$$
\begin{aligned}
& \mathrm{n} 1=\mathrm{x} ; \\
& \mathrm{n} 2=\mathrm{y} ; \\
& \mathrm{R}=\mathrm{z} ; \\
& \mathrm{a}=1 ; \\
& \mathrm{b}=0 ; \\
& \mathrm{c}=((\mathrm{n} 2-\mathrm{n} 1) /(\mathrm{n} 2 *-\mathrm{R})) ; \\
& \mathrm{d}=\mathrm{n} 1 / \mathrm{n} 2 ;
\end{aligned}
$$$$
\text { elseif type }==\text { 'spmi' \%Spherical Mirror with radius of curvature } \mathrm{R} \text { (where input } \mathrm{X}=[\mathrm{R}, 0,0])
$$$$
\mathrm{R}=\mathrm{x}
$$$$
\mathrm{a}=1 \text {; }
$$$$
\mathrm{b}=0 \text {; }
$$$$
\mathrm{c}=-2 / \mathrm{R}
$$$$
\mathrm{d}=1 \text {; }
$$

elseif type $==$ 'tlnl' \%Thermal Lens Negative Lens (tlnl) where input $\mathrm{X}=[\mathrm{x}, \mathrm{dz}, 0]$ \%Reference: Yariv Quantum Electronics pg 108.

$\mathrm{x}=\mathrm{x}$;

$\mathrm{dz}=\mathrm{y}$

temp1 $=\operatorname{sqrt}(\mathrm{x})$;

temp2 $=1 . /(\operatorname{sqrt}(\mathrm{x}))$; 


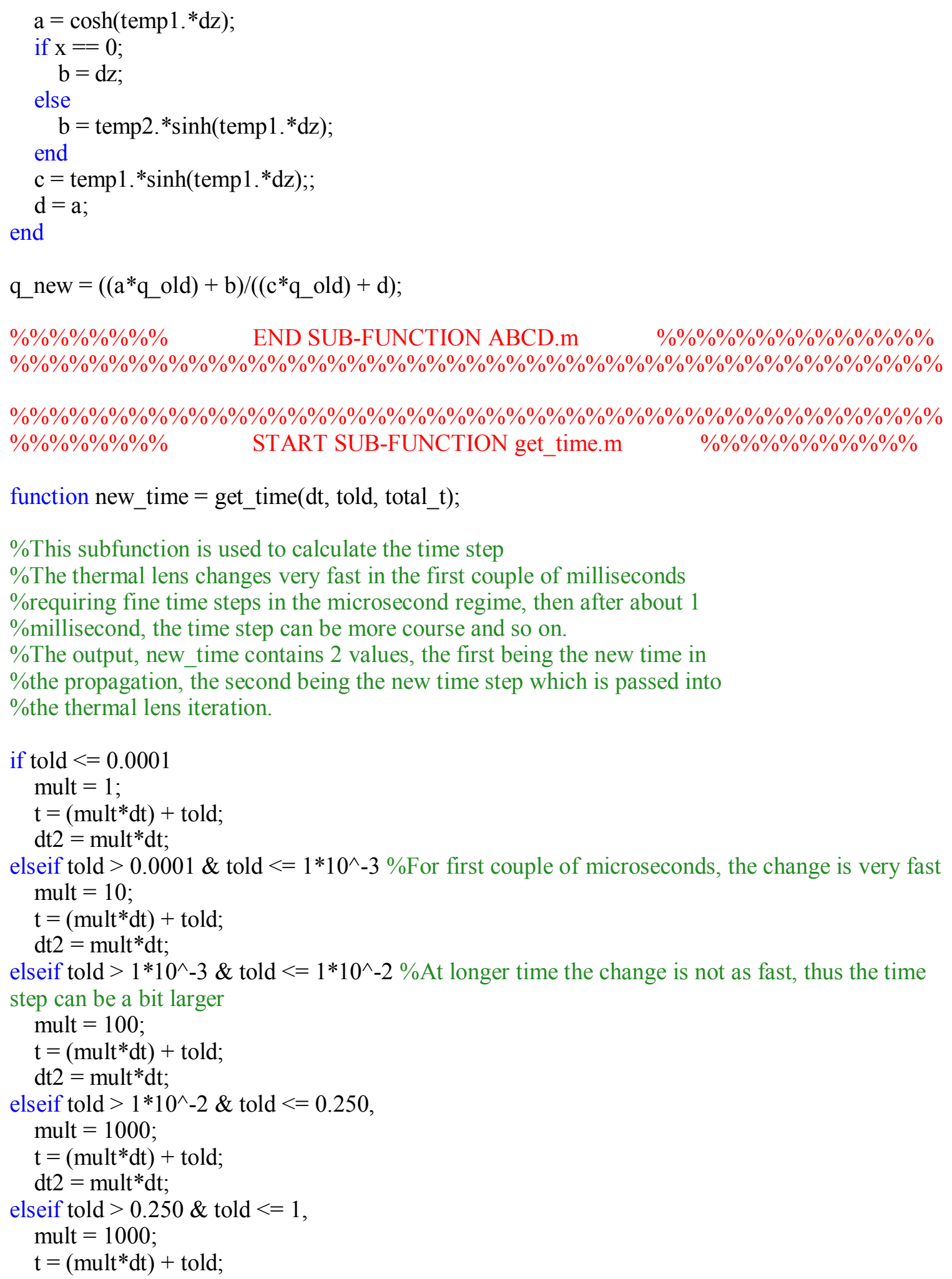




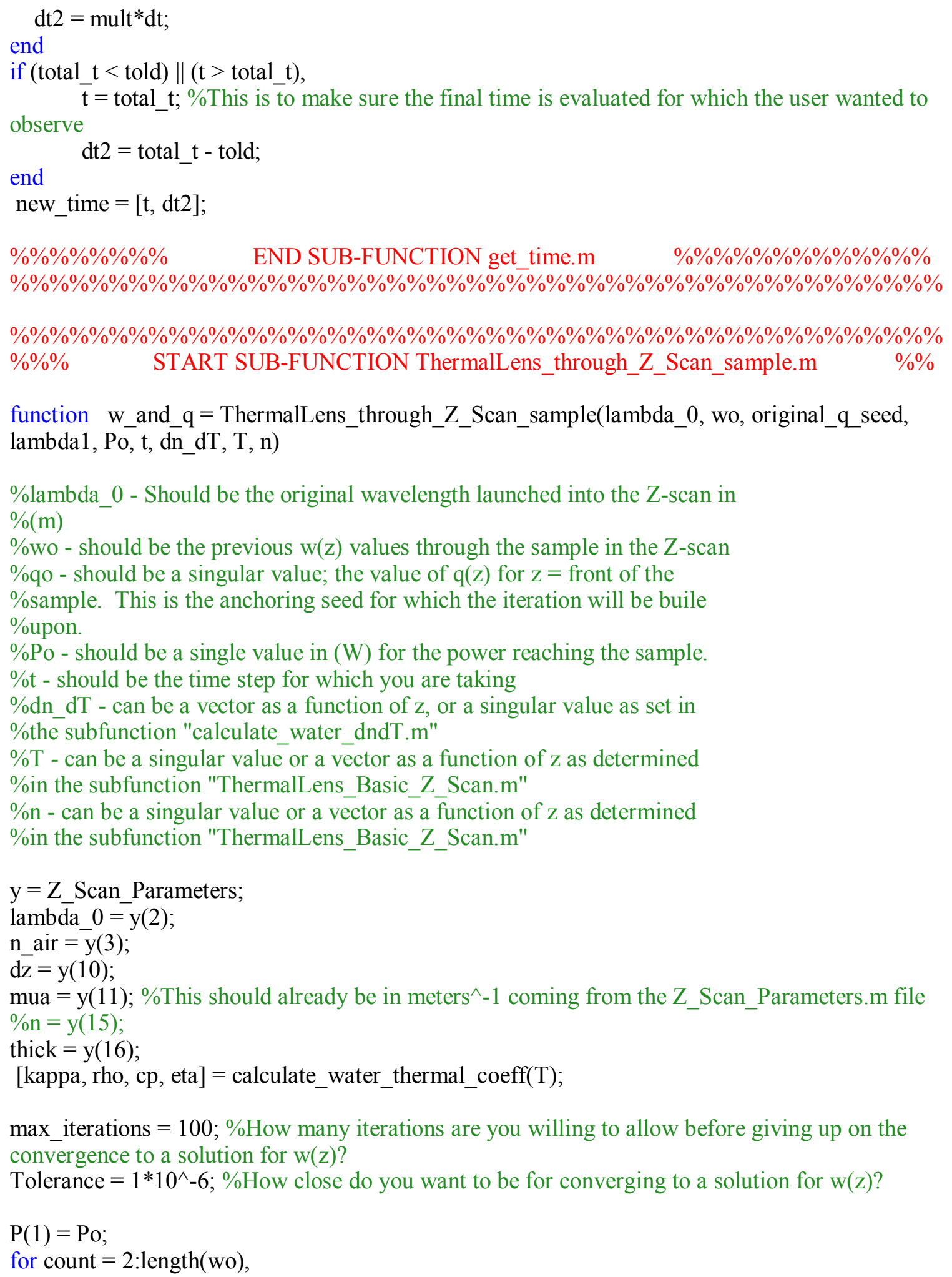




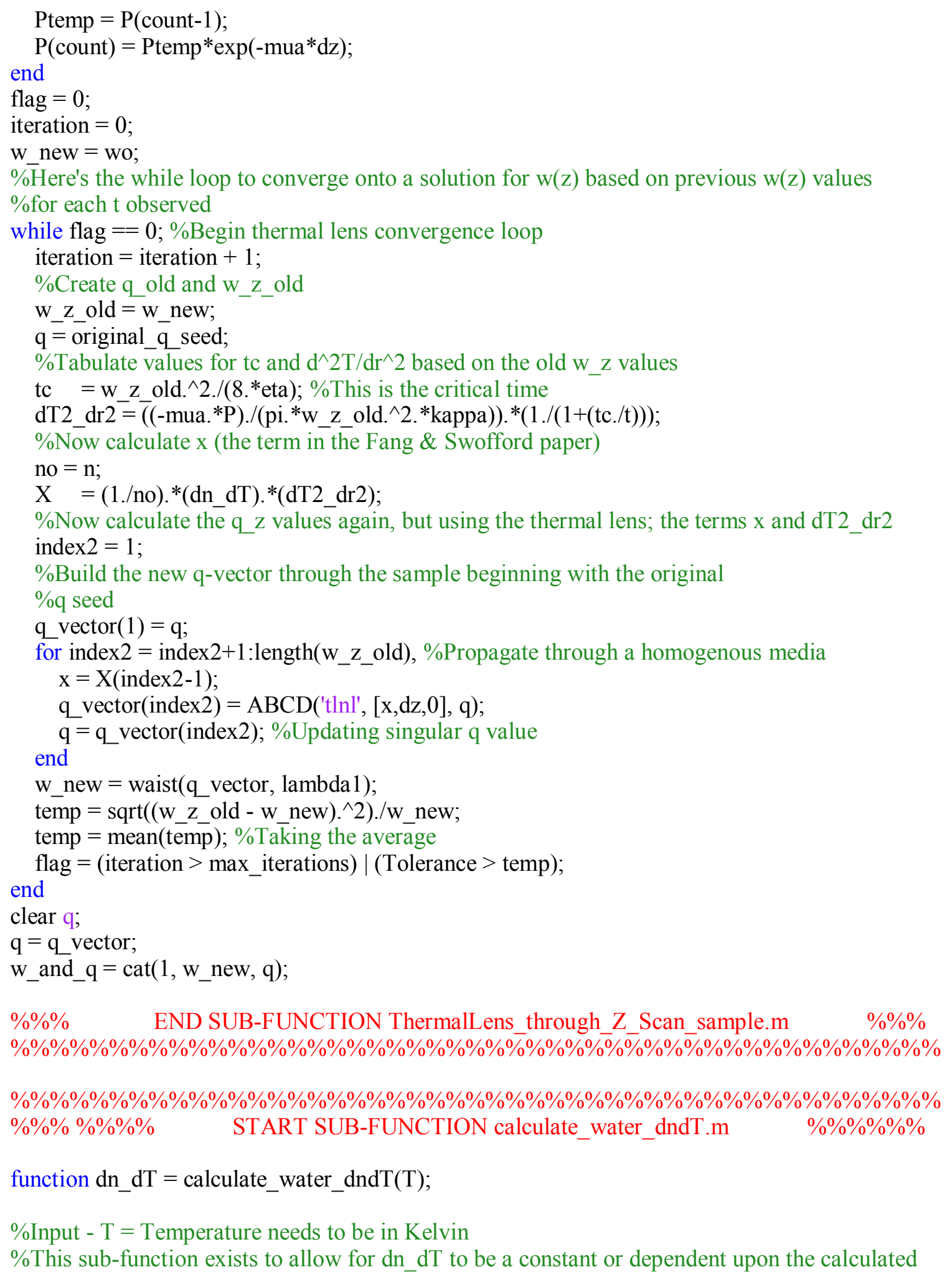


\%temperature, T, rise. For the results in my dissertation, I made dn_dT a constant.

$\mathrm{dn} \_\mathrm{dT}=-4.46 * 10^{\wedge}-5$

$\% \% \% \% \% \% \% \quad$ END SUB-FUNCTION calculate water dndT.m $\quad \% \% \% \% \% \% \% \%$ \%\%\%\%\%\%\%\%\%\%\%\%\%\%\%\%\%\%\%\%\%\%\%\%\%\%\%\%\%\%\%\%\%\%\%\%\%\%\%\%\%\%\%\%\%\%\% 


\section{Appendix B}

\section{LIST OF ACRONYMS}

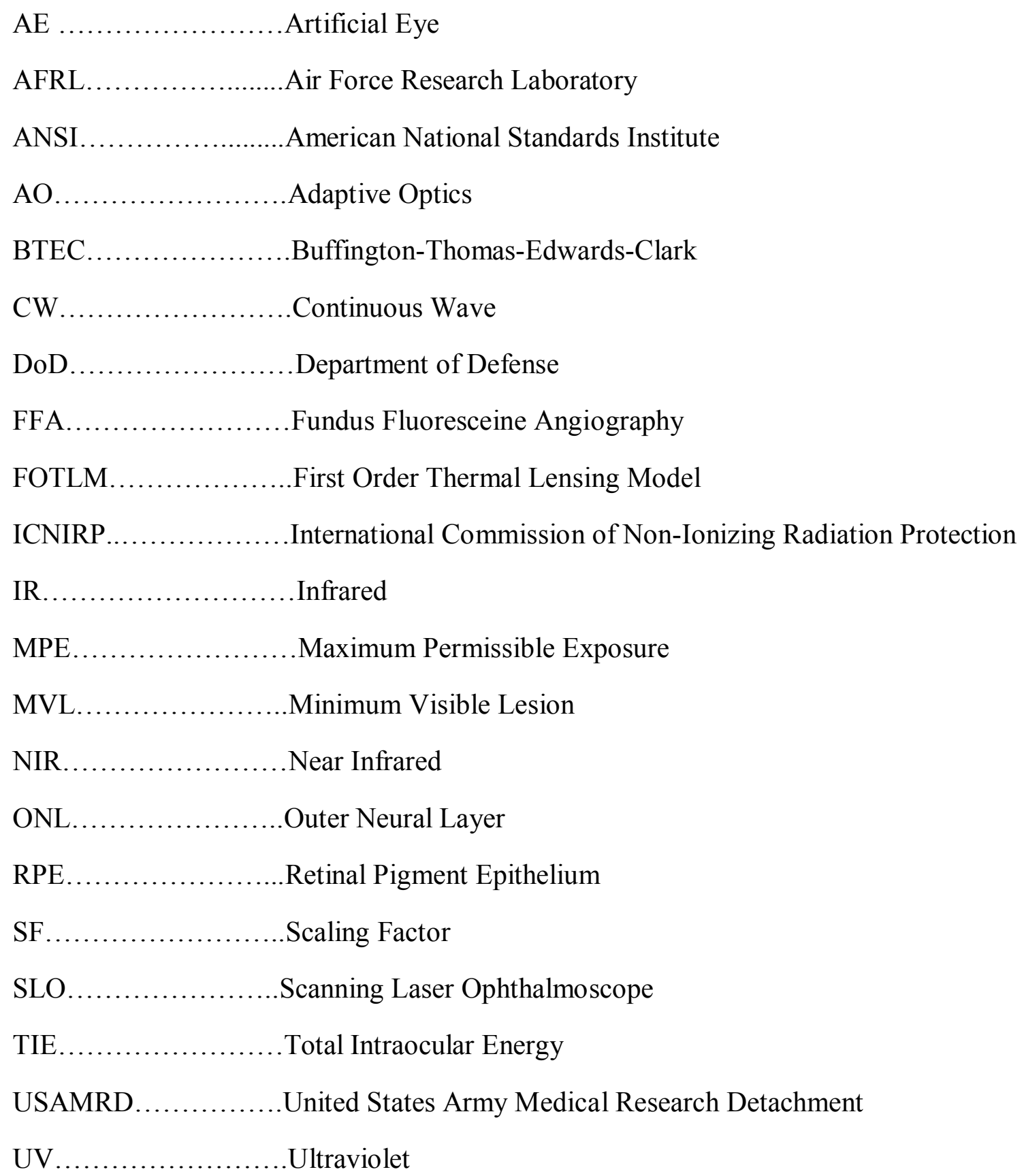




\section{Bibliography}

(1962). "Aberrations of Optical Images" and "The Eye as an Optical System". The Eye: Visual Optics and the Optical Space Sense. H. Davson. New York, Academic Press. 4: $87-131$.

Alfonso, E. F. S., M. A. R. Revert, et al. (1990). "Reduction of convective low-frequency noise in thermal lens spectrometry." Applied Spectroscopy 44(9): 1501-1507.

ANSI (2000). American National Standard for Safe Use of Lasers. Orlando, FL, Laser Institute of America.

Artal, P., A. Benito, et al. (2006). "The human eye is an example of robust optical design." Journal of Vision 6(1): 1-7.

Atchison, D. A. and G. Smith (2002). Optics of the Human Eye. Somerset, England, Elsevier Science.

Atchison, D. A. and G. Smith (2005). "Chromatic dispersions of the ocular media of human eyes." Journal Of The Optical Society Of America. A, Optics, Image Science, and Vision 22(1): 29-37.

Bailey, A. J., T. J. Sims, et al. (1993). "Chemistry of collagen cross-links: Glucosemediated covalent cross-linking of type-IV collagen in lens capsules." Biochemical Journal 296(2): 489-496.

Beck, J. V., K. D. Cole, et al. (1992). Heat Conduction Using Green's Functions. Washington DC, Hemisphere Publishing Corporation.

Bedford, R. E. and G. Wyszecki (1957). "Axial chromatic aberration of the human eye." Journal of the Optical Society of America 47(6): 564-565.

Belanger, P. A. (1991). "Beam propagation and the ABCD ray matrices." Optics Letters 16(4): 196-198.

Birngruber, R., V. P. Gabel, et al. (1983). "Experimental studies of laser thermal retinal injury." Health Physics 44(5): 519-531.

Birngruber, R., F. Hillenkamp, et al. (1985). "Theoretical investigations of laser thermal retinal injury." Health Physics 48(6): 781-796.

Blankenstein, M. F., J. Zuclich, et al. (1986). "Retinal hemorrhage thresholds for Qswitched neodymium-Yag laser exposures." Investigative Ophthalmology and Visual Science 27(7): 1176-1179. 
Boettner, E. A. and J. R. Wolter (1962). "Transmission of the ocular media." Investigative Ophthalmology and Visual Science 1(6): 776-783.

Brancato, R., R. Praresi, et al. (1989). "Histopathology of diode and argon laser lesions in rabbit retina." Investigative Ophthalmology and Visual Science 30(7): 1504-1510.

Buffett, C. E. and M. D. Morris (1983). "Convective effects in thermal lens spectroscopy." Applied Spectroscopy 37(5): 455-458.

Cain, C. P. and G. D. Noojin (1996). A comparison of various probit methods for analyzing yes/no data on a log scale, USAF Armstrong Laboratory, Brooks City-Base, TX: $1-44$.

Cain, C. P., G. D. Noojin, et al. (1997). "Artificial eye for In Vitro experiments of laser light interaction with aqueous media." Journal of Biomedical Optics 2(1): 88-94.

Cain, C. P., G. D. Noojin, et al. (2003). Near-infrared ultrashort pulse laser bioeffects studies, Air Force Research Laboratory: 1-262.

Cain, C. P., C. P. Toth, et al. (1998). "Visible lesion threshold dependency on retinal spot size for ultrashort laser pulses in the near infrared." Proc. of SPIE, San Jose, CA, SPIE. 3254.

Chen, B. (2007). Experimental and modeling study of thermal response of skin and cornea to infrared wavelengths laser irradiation. Biomedical Engineering. Austin, University of Texas. Ph.D.: 240.

Clark, C. D., L. J. Irvin, et al. (2008). BTEC Thermal Model. San Antonio, Air Force Research Laboratory.

Coogan, P. S., W. F. Hughes, et al. (1974). Histologic and spectrophotometric comparisons of the human and rhesus monkey retina and pigmented ocular fundus. Final Report, Contract AF 41(609)-71-C-0006, USAF School of Aerospace Medicine, RushPresbyterian St. Lukes Medical Center.

Denton, M. L., M. S. Foltz, et al. (2009). "Determination of threshold average temperature for cell death in an in vitro retinal model using thermography." Proc. of SPIE, San Jose, CA, SPIE. 7175.

Denton, M. L., M. S. Foltz, et al. (2007). "Damage thresholds for cultured retinal pigment epithelial cells exposed to lasers at $532 \mathrm{~nm}$ and $458 \mathrm{~nm}$." Journal of Biomedical Optics 12(3): 0340301-8.

Denton, M. L., M. S. Foltz, et al. (2008). "In vitro model that approximates retinal damage threshold trends." Journal of Biomedical Optics 13(5): 0540141-6. 
Eldred, G. and M. Katz (1988). "Fluorophores of the human retinal pigment epithelium: Separation and spectral characterization." Experimental Eye Research 47(1): 71-86.

Fang, H. L. and R. Swofford (1979). "Analysis of the thermal lensing effect for an optically thick sample-A revised model." Journal of Applied Physics 50(11): 6609-6615.

Fernandes, A., D. V. Bradley, et al. (2003). "Ocular measurements throughout the adult life span of rhesus monkeys." Investigative Ophthalmology and Visual Science 44(6): 2373-2380.

Fernandez, E. J., A. Unterhuber, et al. (2006). "Chromatic aberration correction of the human eye for retinal imaging in the near infrared." Optics Express 14(13): 6213-6225.

Fernández, E. J., A. Unterhuber, et al. (2005). "Ocular aberrations as a function of wavelength in the near infrared measured with a femtosecond laser." Optics Express 13(2): 400-409.

Finney, D. J. (1971). Probit Analysis. Cambridge, England, University Press.

Fishkin, N. E., J. R. Sparrow, et al. (2005). "Isolation and characterization of a retinal pigment epithelial cell fluorophore: An all-trans-retinal dimer conjugate." Proceedings of the National Academy of Sciences of the United States of America 102(20): 7091-7096.

Franko, M. and C. D. Tran (1996). "Analytical thermal lens instrumentation." Review of Scientific Instrumentation 67(1): 1-18.

Gabel, V. P., R. Birngruber, et al. (1978). Visible and near infrared light absorption in pigment epithelium and choroid. XXIII Concilium Ophthalmologicum (Reprinted from the International Congress Series No. 450). K. Shimizu. Kyoto, Excerpta Medica, Amsterdam-Oxford: 658-662.

Gabel, V. P., R. Birngruber, et al. (1976). "Die lichtabsorption am augenhintergrund." GSF-Bericht A 55 .

Geeraets, W. J., R. C. Williams, et al. (1960). "The loss of light energy in retina and choroid." Archives of Ophthalmology 64(1): 158-167.

Geeraets, W. J., R. C. Williams, et al. (1962). "The relative absorption of thermal energy in retina and choroid." Investigative Ophthalmology and Visual Science 1(3): 340-347.

Gerstman, B. S. and R. D. Glickman (1999). "Activated rate processes and a specific biochemical mechanism for explaining delayed laser induced thermal damage to the retina." Journal of Biomedical Optics 4(3): 345-351. 
Glickman, R. A. (2002). "Phototoxicity to the retina: Mechanisms of damage." International Journal of Toxicology 21(6): 473-490.

Gordon, J. P., R. C. Leite, et al. (1965). "Long transient effects of lasers with inserted liquid samples." Journal of Applied Physics 36(3): 3-8.

Hale, G. M. and M. R. Querry (1973). "Optical constants of water in the 200-nm to 200$\mu \mathrm{m}$ wavelength region." Applied Optics 12(3): 555-563.

Harvey, A. H., J. S. Gallagher, et al. (1998). "Revised formulation for the refractive index of water and steam as a function of wavelength, temperature and density." Journal of Physical and Chemical Reference Data 27: 761-774.

Henderson, R. and K. Schulmeister (2004). Laser Safety. Philadelphia, PA, Institute of Physics Publishing.

Hughes, A. (1972). "A schematic eye for the rabbit." Vision Research 12(1): 123-138.

ICNIRP (2000). "Revision of guidelines on limits of exposure to laser radiation of wavelengths between $400 \mathrm{~nm}$ and 1.4 microns." Health Physics 79(4): 431-440.

Kim, C. B. and C. B. Su (2004). "Measurement of the refractive index of liquids at 1.3 and 1.5 micron using a fibre optic Fresnel ratio meter." Measurement Science and Technology 15(x): 1683-1686.

Kovsh, D., S. Yang, et al. (1999). "Nonlinear optical beam propagation for optical limiting." Applied Optics 38(24): 5168-5180.

Kovsh, D. I., D. J. Hagan, et al. (1999). "Numerical modeling of thermal refraction in liquids in the transient regime." Optics Express 4(8): 315-327.

Kozich, V. P., F. E. Hernandez, et al. (1995). "Pulse-induced thermal lensing in Kerr media." Applied Spectroscopy 49(12): 1804-1808.

Lange, M., G. Pitz, et al. (2005). "Electric discharge pumped oxygen-iodine laser kinetics." Journal of Directed Energy 1: 219-232.

Lappin, P. W. and P. S. Coogan (1970). "Histologic evaluation of ophthalmoscopically subvisible retinal laser exposures." Investigative Ophthalmology and Visual Science 9(7): $537-542$.

Lee, B., M. Litt, et al. (1992). "Rheology of the vitreous body. Part 1: Viscoelasticity of human vitreous." Biorheology 29(5 \& 6): 521-533. 
Li, G., H. Zwick, et al. (2000). "On the use of schematic eye models to estimate retinal image quality." Journal of Biomedical Optics 5(3): 307-314.

Liang, J., B. Grimm, et al. (1994). "Objective measurement of wave aberrations of the human eye of a Hartmann-Shack wave-front sensor." Journal of the Optical Society of America, A 11(7): 1949-1957.

Lin, W. C. (1997). Dynamics of tissue optics during laser heating. Biomedical Engineering. Austin, TX, University of Texas. Ph.D.

Lin, W. C., M. Motamedi, et al. (1995). "Nonlinear optical behavior of ocular tissue during laser irradiation." Applied Optics 34(34): 7979-7985.

Liou, H. L. and N. A. Brennan (1997). "Anatomically accurate, finite model eye for optical modeling." J Opt Soc Am A Opt Image Sci Vis 14(8): 1684-95.

Lund, B. J. (2006). "Laser retinal thermal damage threshold: impact of small scale ocular motion." Journal of Biomedical Optics 11(6): 0640331-8.

Lund, D. J. and P. Edsall (1999). "Action spectrum for retinal thermal injury." Proc. of SPIE, San Jose, CA, SPIE. 3591.

Lund, D. J., P. Edsall, et al. (2000). "Spectral dependence of retinal thermal injury." Proc. of SPIE, San Jose, CA, SPIE. 3902.

Lund, D. J., P. Edsall, et al. (2005). "Wavelength dependence of laser-induced retinal injury." Proc. of SPIE, Bellingham, WA, SPIE. 5688.

Lund, D. J., P. Edsall, et al. (2008). "Spectral dependence of retinal thermal injury." Journal of Laser Applications 20(2).

Lund, D. J., P. Edsall, et al. (2007). "Variation of laser-induced retinal injury thresholds with retinal irradiated area: 0.1-s duration, 514-nm exposures." Journal of Biomedical Optics 12(2): 024023.

Lund, D. J., P. R. Edsall, et al. (1998). "Bioeffects of near-infrared lasers." Journal of Laser Applications 10(3): 140-143.

Lund, D. J., B. E. Stuck, et al. (1981). Biological Research in Support of Project MILES. San Francisco, CA, Letterman Army Institute of Research, Presidio: 1-54.

Maher, E. F. (1978). Transmission and absorption coefficients for the ocular media of the Rhesus monkey, USAF School of Aerospace Medicine: 1-104. 
Mansell, J. D., J. Hennawi, et al. (2001). "Evaluating the effect of transmissive optic thermal lensing on laser beam quality with a Shack-Hartmann wave-front sensor." Applied Optics 40(3): 366-374.

Marquet, L. C. and J. Hardy (1978). "Historical review of adaptive optics technology." Washington D.C., SPIE.

Mikesell., G. W., Jr. (1978). "Corneal temperatures - A study of normal and laser injured corneas in the Dutch Belted Rabbit." American Journal of Optometry and Physiological Optics 55(2): 108-115.

Motamedi, M., A. J. Welch, et al. (1988). "Thermal lensing in biologic medium." IEEE Journal of Quantum Electronics 24(4): 693-696.

Nofsinger, J. B., S. E. Forest, et al. (1999). "Explanation for the disparity among absorption and action spectra of eumelanin." Journal of Physical Chemistry B 103(51): $11428-11432$

Nofsinger, J. B. and J. D. Simon (2001). "Radiative relaxation of sepia eumelanin is affected by aggregation." Photochemistry and Photobiology 74(1): 31-37.

Okuno, T., M. Kojima, et al. (2005). "Temperature rises in the crystalline lens from focal irradiation." Health Physics 88(3): 214-222.

Pearce, J. and S. Thomsen (1995). Rate process analysis of thermal damage. OpticalThermal Response of Laser-Irradiated Tissue. A. J. Welch and M. J. C. Van Gemert, Plenum Publishing: 594.

Pearce, J. A. and S. Thomsen (2003). "Thermal damage parameters from laser coagulation experiments." Proc. of SPIE, San Jose, CA, SPIE. 4954.

Peri, D., J. Turetz, et al. (2006). "Optical system for exposure of rabbit eyes to laser light and in-situ assessment of retinal damage." Proc. of SPIE, SPIE. 6138.

Polhamus, G. and A. J. Welch (1975). "Effect of pre-exposure fundus temperature on threshold lesion temperatures in the laser-irradiated rabbit retina." Investigative Ophthalmology 14(7): 562-565.

Porras, M. A., J. Alda, et al. (1993). "Nonlinear propagation of arbitrary laser beams by means of the generalized ABCD formalism." Applied Optics 32(30): 5885-5892.

Priebe, L. A. and A. J. Welch (1978). "Asymptotic rate process calculations of thermal injury to the retina following laser irradiation." Journal of Biomechanical Engineering 100(1): 49-54. 
Roorda, A. (2002). Human Visual System - Image Formation. Encyclopedia of Imaging Science and Technology. J. P. Hornak. New York, John Wiley \& Sons. 1: 539-557.

Schiebener, P., J. Straub, et al. (1990). "Refractive index of water and steam as function of wavelength, temperature and density." Journal of Physical and Chemical Reference Data 19(3): 677-717.

Sheik-Bahae, M., A. A. Said, et al. (1990). "Sensitive measurement of optical nonlinearities using a single beam." IEEE Journal of Quantum Electronics 26(4): 760769.

St. John, W. D., B. Taheri, et al. (1992). "Time-dependent thermal lensing in lead oxidemodified silicate glass." Journal of the Optical Society of America, B 9(4): 610-616.

Strauss, O. (2005). "The Retinal Pigment Epithelium in Visual Function." Physiological Review 85(3): 845-881.

Stuck, B. E., D. J. Lund, et al. (1980). Ocular effects of laser radiation from 1.06 to 2.06 $\mu \mathrm{m}$. Ocular Effects of Non-Ionizing Radiation. M. L. Wolbarsht and D. H. Sliney. San Francisco, CA, Letterman Army Institute of Research. 229.

Sugita, N., T. Osa, et al. (2009). "Analysis and estimation of cutting-temperature distribution during end milling in relation to orthopedic surgery." Medical Engineering and Physics 31(1): 101-107.

Swann, D. A., I. J. constable, et al. (1972). "Vitreous structure, III. Composition of bovine vitreous collagen." Investigative Ophthalmology 11(9): 735-738.

Tabernero, J., P. Piers, et al. (2006). "Predicting the optical performance of eyes implanted with IOLS to correct spherical aberration." Investigative Ophthalmology and Visual Science 47(10): 4651-4658.

Thibos, L. N. (2000). "Principles of Hartmann-Shack aberrometry." Journal of Refractive Surgery 16: 563-565.

Thibos, L. N. (2001). "Wavefront data reporting and terminology." Journal of Refractive Surgery 17: S578-S583.

Thibos, L. N., X. Hong, et al. (2002). "Statistical variation of aberration structure and image quality in a normal population of healthy eyes." Journal of the Optical Society of America A 19(12): 2329-2348.

Thomas, R. J., R. L. Vincelette, et al. (2005). "A first order model of thermal lensing of laser propagation in the eye and implications for laser safety." International Laser Safety Conference, Marina del Rey, CA, Laser Institute of America. 
Thomas, R. J., R. L. Vincelette, et al. (2007). "Propagation effects in the assessment of laser damage thresholds to the eye and skin." Proc. of SPIE, San Jose, CA, SPIE. 6435.

van den Berg, T. J. T. P. and H. Spekreijse (1997). "Near infrared light absorption in the human eye media." Vision Research 37(2): 249-253.

van Gemert, M. J. C. and A. J. Welch (1995). Approximate solutions for heat conduction: time constants. Optical-Thermal Response of Laser-Irradiated Tissue. A. J. Welch and M. J. C. van Gemert, Plenum Publishing.

van Nimwegen, S. A., H. F. L'Eplattenier, et al. (2009). "Nd:YAG surgical laser effects in canine prostate tissue: temperature and damage distribution." Physics in Medicine and Biology 54(1): 29-44.

Vincelette, R. L., J. W. Oliver, et al. (2009). "Thermal lensing from near-infrared laser radiation in an artificial eye." Proc. of SPIE, San Jose, CA, SPIE. 7175.

Vincelette, R. L., B. A. Rockwell, et al. (2009). "Trends in retinal damage thresholds from 100-ms near-infrared laser radiation exposures: a study at 1110, 1130, 1150 and 1319 nm." Lasers in Surgery and Medicine 41(5): Accepted for pub. April 7.

Vincelette, R. L., B. A. Rockwell, et al. (2008). "Thermal lensing in ocular media exposed to continuous-wave near-infrared radiation: $1150-1350 \mathrm{~nm}$ region." Journal of Biomedical Optics 13(5): 054005.

Vincelette, R. L., R. J. Thomas, et al. (2009). "A first-order model of thermal lensing in a virtual eye." Journal of the Optical Society of America A 26(3): 548-558.

Vincelette, R. L., R. J. Thomas, et al. (2006). "A comparison of a first-order thermal lensing model to a closed aperture Z-scan for the propagation of light in ocular media." Proc. of SPIE, San Jose, CA, SPIE. 6084.

Vincelette, R. L., R. J. Thomas, et al. (2007). "Thermal lensing in the ocular media." Proc. of SPIE, San Jose, CA, SPIE. 6435.

Vitkin, A., J. Woosley, et al. (1994). "Optical and thermal characterization of natural (Sepia officinalis) melanin." Photochemistry and Photobiology 59(4): 455-462.

Wagner, W. and A. Kruse (1998). Properties of Water and Steam: The Industrial Standard IAPWS-IF97 for the Thermodynamic Properties and Supplementary Equations for Other Properties. New York, Springer-Verlag: 150.

Wald, G. and D. R. Griffen (1947). "The change in refractive power of the eye in dim and bright light." Journal of the Optical Society of America 37(5): 321-326. 
Walsh, J. T. (1995). Pulsed Laser Angioplasty: A Paradigm for Tissue Ablation. OpticalThermal Response of Laser-Irradiated Tissue. A. J. Welch and M. J. C. van-Gemert. New York, Plenum Press: 889-893.

Weisstein, E. W. (2009). "Zernike Polynomial." Retrieved Jan. 15, 2009, from http://mathworld.wolfram.com/ZernikePolynomial.html.

Welch, A. J. and G. D. Polhamus (1984). "Measurement and prediction of thermal injury in the retina of the rhesus monkey." IEEE Transactions on Biomedical Engineering 31(10): 633-644.

Westheimer, G. (1980). The Eye: Including central nervous system control of eye movements. St. Louis, MO, C.V. Mosby Company.

Wyld, H. A. (1976). Mathematical Method for Physics. Reading, MA, W. A. Benjamin, Inc.

Yariv, A. (1989). Quantum Electronics. New York, John Wiley and Sons.

Yeh, P. C. and D. T. Azar (2005). Wavefront-Guided Custom LASIK and LASEK: Techniques and Outcomes. Smolin and Thoft's The Cornea: Scientific Foundations and Clinical Practice. C. S. Foster, D. T. Azar and C. H. Dohlman. Philadelphia, Lippincott Williams and Wilkins: 1256-1268.

Yumita, A., S. Shirato, et al. (1986). "Retinal damage threshold of ophthalmic Qswitched ND-YAG laser in monkey eyes." Japanese Journal of Ophthalmology 30(1): 100-115.

Zohner, J. J., C. D. Clark, et al. (2008). "Incorporation of refractive index gradients in the solution of the radiative transport equation." Proc. of SPIE, San Jose, CA, SPIE. 6854.

Zuclich, J. A., M. F. Blankenstein, et al. (1984). "Corneal damage induced by pulsed CO2 laser radiation." Health Physics 47(6): 829-835.

Zuclich, J. A., P. R. Edsall, et al. (2000). "Variation of laser induced retinal-damage threshold with retinal image size." Journal of Laser Applications 12(2): 74-80.

Zuclich, J. A., D. A. Gagliano, et al. (1995). "Ocular effects of penetrating IR laser wavelengths." Proc. of SPIE, San Jose, CA, SPIE. 2391.

Zuclich, J. A., D. J. Lund, et al. (2002). "New data on the variation of laser-induced retinal damage threshold with retinal image size." Proceedings of the Laser Bioeffects Meeting, Paris, France. 
Zuclich, J. A., D. J. Lund, et al. (1999). "Experimental study of the variation of laserinduced retinal damage threshold with retinal image size." Molecular Crystals and Liquid Crystals Science and Technology Section B: Nonlinear Optics 21(1-4): 19-28.

Zuclich, J. A., D. J. Lund, et al. (2001). "Highpower lasers in the 1.3- to 1.4- $\mu \mathrm{m}$ wavelength range: ocular effects and safety standard implications." Proc. of SPIE, San Jose, CA, SPIE. 4246.

Zuclich, J. A., D. J. Lund, et al. (2007). "Wavelength dependence of ocular damage thresholds in the near-IR to far-IR transition region: Proposed revisions to MPEs." Health Physics 92(1): 15-23.

Zuclich, J. A., D. J. Lund, et al. (2004). Ocular effects and safety standard implications for high-power lasers in the 1.3-1.4 $\mu \mathrm{m}$ wavelength range. Technical Report. San Antonio, Air Force Research Laboratory: 1-16.

Zuclich, J. A., S. Schuschereba, et al. (1996). "Comparing laser-induced retinal damage from IR wavelengths to that from visible wavelengths." Proc. of SPIE, San Jose, CA, SPIE. 2674.

Zuclich, J. A., S. T. Schuschereba, et al. (1997). "A comparison of laser-induced retinal damage from infrared wavelengths to that from visible wavelengths." Lasers and Light in Ophthalmology 8(1): 15-29.

Zuclich, J. A., H. T. Zwick, et al. (1998). "Ophthalmoscopic and pathologic description of ocular damage induced by infrared laser radiation." Journal of Laser Applications 10(3): 114-120. 


\section{Vita}

Rebecca Lee Vincelette was born in Laramie, WY, 1979, and moved around between ID, ND, KS, WY and finally CO, where she attended Green Mountain High School in Lakewood. In 1997, she went to the University of Wyoming and completed her double majors in Electrical Engineering and Chemistry in 2001. In 2002 she moved to Austin, Texas to attend graduate school at the University of Texas. Upon completing her M.S. degree in Biomedical Engineering in 2004, she began a three-year active-duty service tour as an officer in the United States Air Force. In 2007, she separated from the Air Force to return to graduate school full-time at UT Austin to pursue her Ph.D. in Biomedical Engineering supported by the Consortium Research Fellows Program in Arlington, VA. She currently lives just outside of San Antonio, TX, with her husband and two dogs.

Permanent address: 13865 E. $105^{\text {th }}$ Ave., Commerce City, CO, 80022

This manuscript was typed by the author. 